University of Szeged

Faculty of Pharmacy

Department of Pharmaceutical Technology and Regulatory Affairs

Head: Dr. Ildikó Csóka PhD

PhD thesis

Optimization of a combined wet milling process to produce nanosuspension and its transformation into surfactant-free solid compositions to increase the product stability and drug bioavailability

By

Csaba Bartos

Pharmacist

Supervisor:

Prof. Dr. Piroska Szabó-Révész DSc

SZEGED

2019 


\section{PUBLICATIONS RELATED TO THE SUBJECT OF THE THESIS}

1. Bartos C, Szabó-Révész P, Bartos Cs, Katona G, Jójárt-Laczkovich O, Ambrus R. The effect of an optimized wet milling technology on the crystallinity, morphology and dissolution properties of micro- and nanonized meloxicam. Molecules 21 (2016) 507-518.

IF: 3.098 Q1

2. Bartos Cs, Jójárt-Laczkovich O, Katona G, Budai-Szücs M, Ambrus R, Bocsik A, Gróf I, Deli M A, Szabó-Révész P. Optimization of a combined wet milling process in order to produce poly(vinyl alcohol) stabilized nanosuspension. Drug. Des. Dev. Ther. 12 (2018) 1567-1580.

IF: 2.935 Q1

3. Bartos Cs, Jójárt-Laczkovich O, Regdon G Jr, Szabó-Révész P. Robustness testing of milling process, analyzing the particle size distribution and crystallinity of the milled samples. J. Therm. Anal. Cal. (2019) DOI: 10.1007/s10973-019-08395-2 IF: 2.209 Q2

4. Bartos Cs, Ambrus R, Katona G, Gáspár R, Márki Á, Ducza E, Ivanov A, Tömösi F, Janáky T, Szabó-Révész P. Transformation of meloxicam containing nanosuspension into surfactant-free solid compositions to increase the product stability and drug bioavailability for rapid analgesia. Drug Des. Dev. Ther. (accepted for publication) IF: 3.208 Q1

\section{OTHER PUBLICATION}

Bartos C, Ambrus R, Bartos Cs, Szabó-Révész P. Preparation and comparison of methacrylate copolymer-based microparticles for intranasal application. Acta Pharmaceutica Hungarica (2019) 


\section{PRESENTATIONS RELATED TO THE THESIS}

Bartos Cs, Szabó-Révész P, Jójárt-Laczkovich O. Optimization of particle size of meloxicam with combined wet milling process. $11^{\text {th }}$ Central European Symposium on Pharmaceutical Technology, 2016 September 22-24, Belgrade, Serbia.

Bartos Cs, Jójárt-Laczkovich O, Szabó-Révész P. Comparison study of nanonized meloxicam and its potassium salt containing peroral dosage form with fast release. $7^{\text {th }}$ BBBB International Conference, 2017 October 5-7, Balatonfüred, Hungary.

Bartos Cs, Regdon G jr., Jójárt-Laczkovich, O, Szabó-Révész, P. Scaling up of a combined wet milling process. $12^{\text {th }}$ Central European Symposium on Pharmaceutical Technology, 2018 September 20-22, Szeged, Hungary.

Bartos, Cs, Szabó-Révész, Piroska: Formulation of a solid oral drug delivery systems containing nanosuspension produced by combined wet milling technique. Symposium of Young Researchers on Pharmaceutical Technology, Biotechnology and Regulatory Science. p. 23. 2019 January 31, Szeged, Hungary.

Bartos Cs, Ambrus R, Szabó-Révész P. Transformation of nanosuspension as an intermediate product into solid dosage forms to improve the drug bioavailability. $3^{\text {rd }}$ European Conference on Pharmaceutics, 2019 March 25-26, Bologna, Italy. 
1. INTRODUCTION

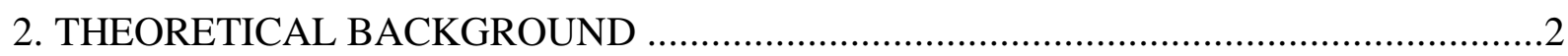

2.1. Strategies to increase the dissolution/solubility of poorly water-soluble active agent....2

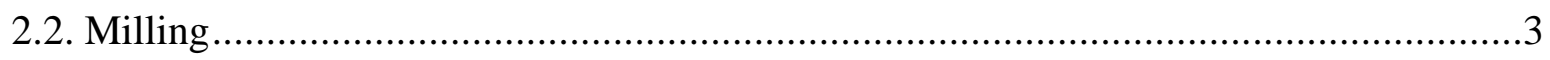

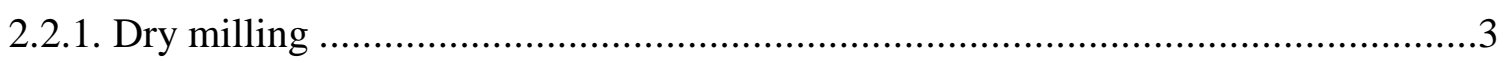

2.2.2. Wet milling ..............................................................................................

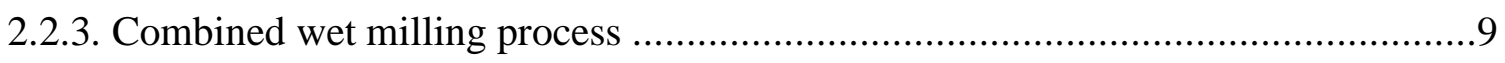

2.3. Transformation of nanosuspension as an intermediate product into solid state product

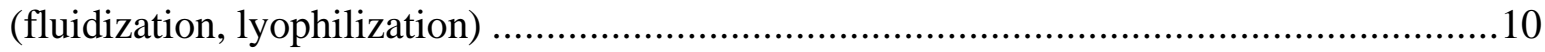

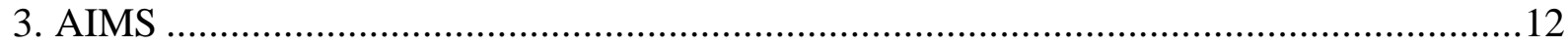

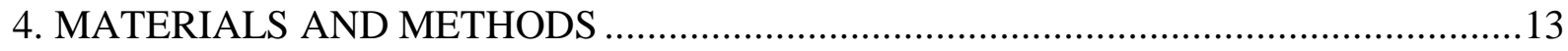

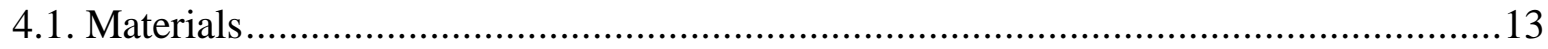

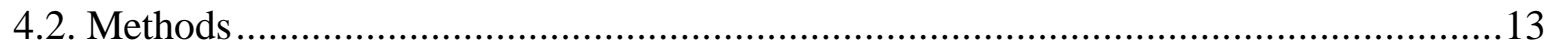

4.2.1. Optimization of combined wet-milling process............................................. 13

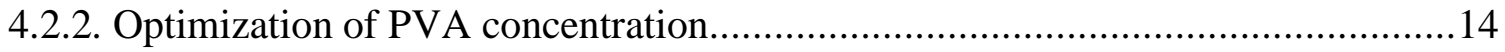

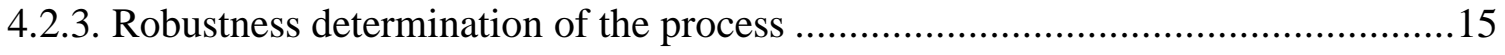

4.2.4. Transformation of nanosuspension into solid compositions ...............................15

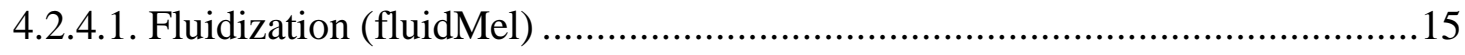

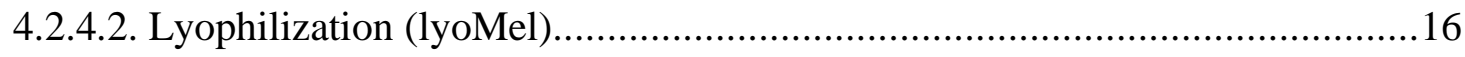

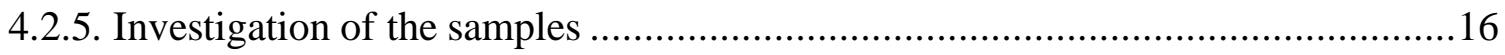

4.2.5.1. Particle size distribution measurements ................................................ 16

4.2.5.2. Image analysis (scanning electron microscopy - SEM) ..............................17

4.2.5.3. X-ray powder diffraction analysis (XRPD) ….......................................... 17

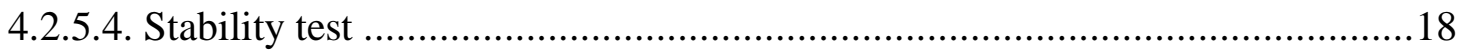

4.2.5.5. Differential scanning calorimetry (DSC)................................................. 18

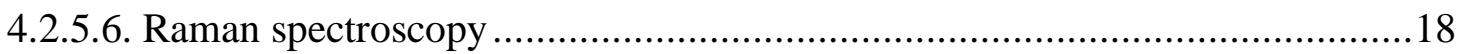

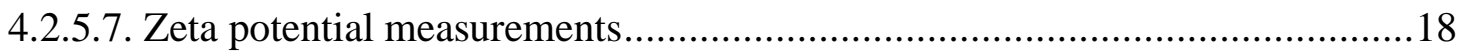

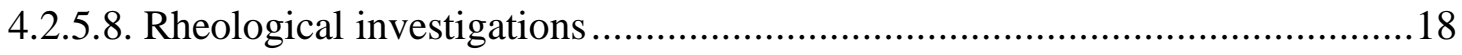

4.2.5.9. Solubility testing of $\mathrm{Mel}$ in the pre-dispersions ........................................ 19

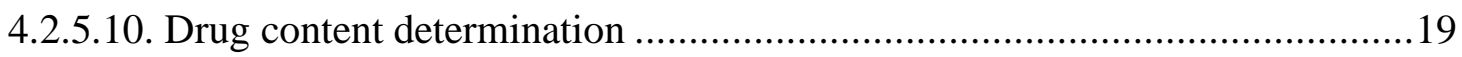

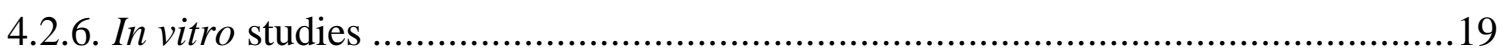

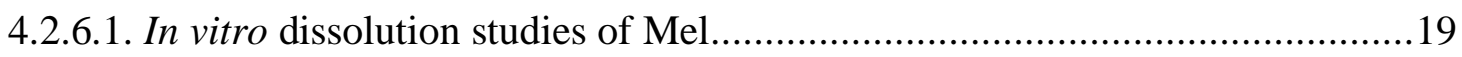

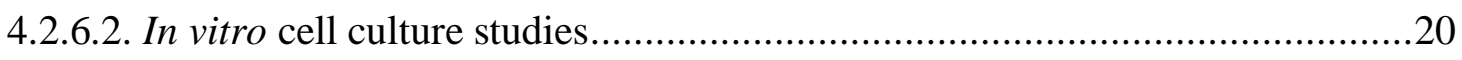

4.2.6.2.1. Human Caco-2 intestinal epithelial cell line ......................................20 
4.2.6.2.2. Cell viability measurement by impedance .20

4.2.6.2.3. Permeability study on cell culture model...........................................20

4.2.6.3. Holding time determination of Mel in the pre-dispersions ..........................21

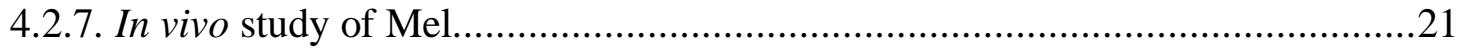

4.2.7.1. In vitro-in vivo correlation calculation ........................................................23

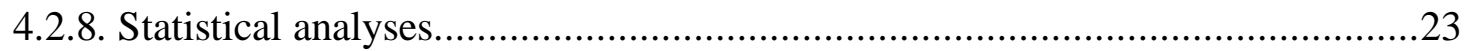

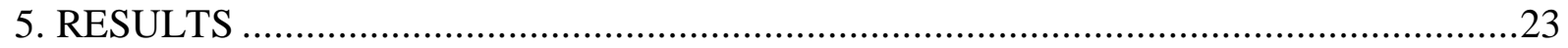

5.1. Optimization of the combined wet milling process .............................................23

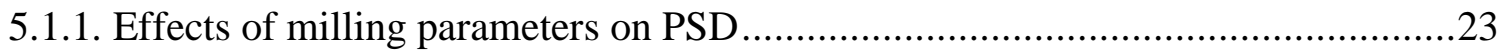

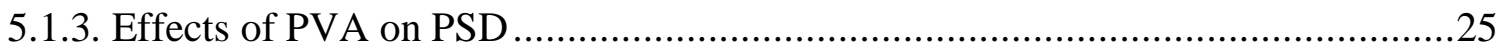

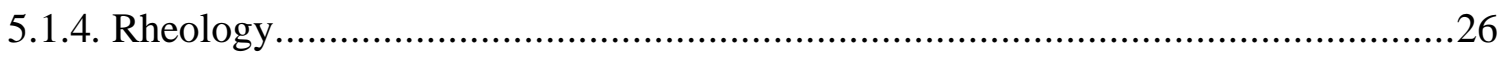

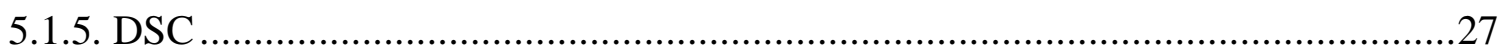

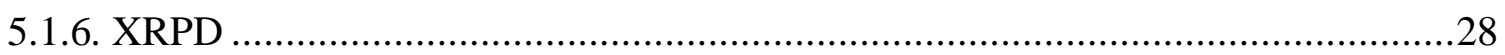

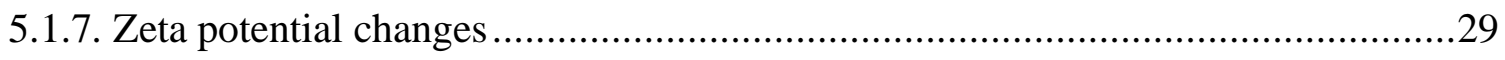

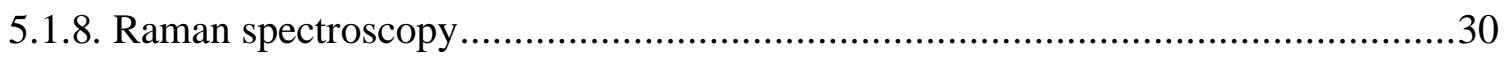

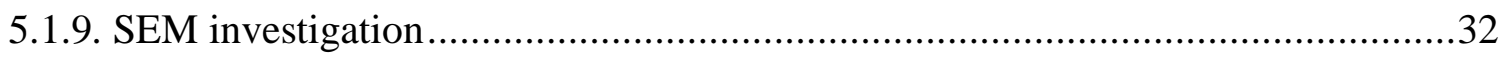

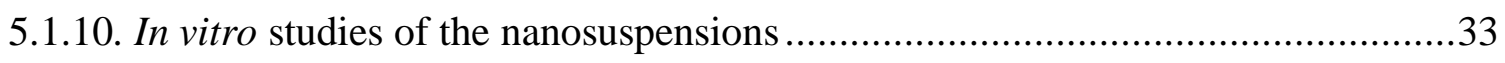

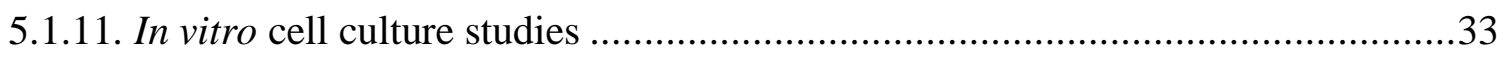

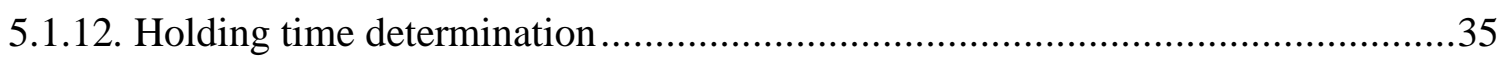

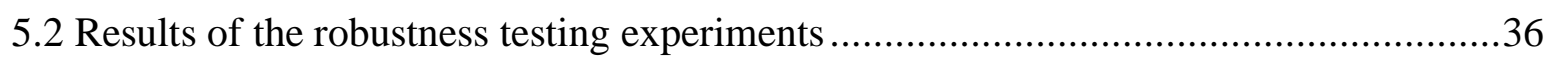

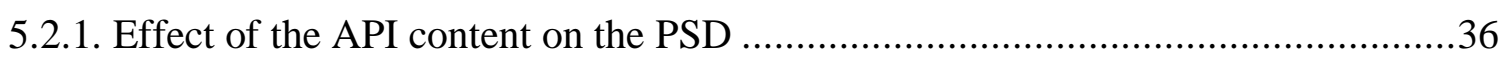

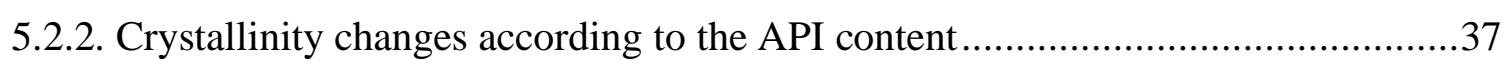

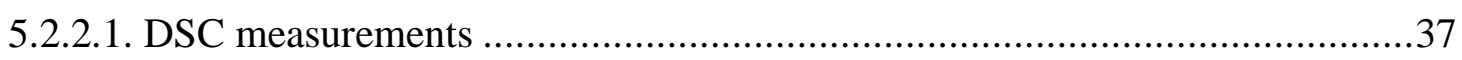

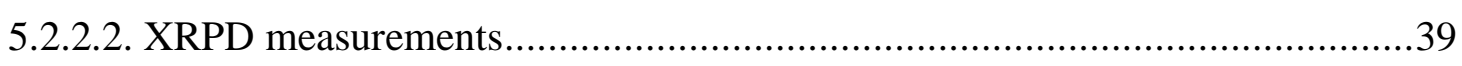

5.2.2.3. Correlation between the two analytical methods ......................................40

5.3. Results of the solid-state product characterizations............................................ 41

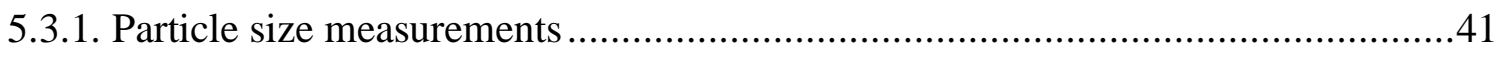

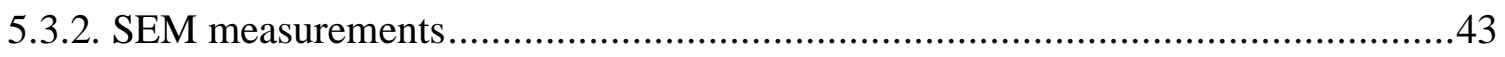

5.3.3. DSC measurements, crystallinity determination .............................................44

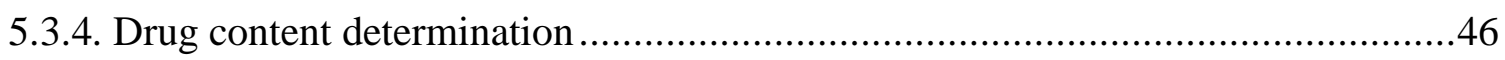

5.3.5. Solubility testing of MEL in the samples ....................................................46

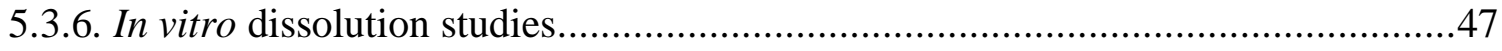

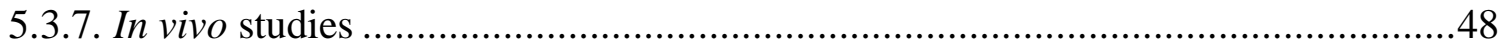

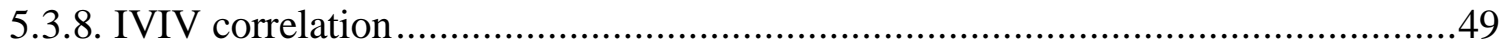

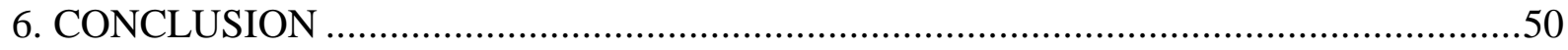


Abbreviations

$\Delta \mathrm{HA}$

$\Delta \mathrm{Hd}$

$\Delta \mathrm{HP}$

$\Delta \mathrm{HS}$

$\Delta \mathrm{HT}$

API

AUC

C

Cryst. \%

$\mathrm{d}(0.1)$

$\mathrm{d}(0.5)$

$\mathrm{d}(0.9)$

E

fluidMel

HPC

$\mathrm{HPH}$

HPMC

LD50

lyoMel

Mel

MelD

Mel-PVA PM

microMel

nanoMel

n

NSAID

PEG

PM

Poloxamer amorphous material formation energy

relative lattice distortion energy

new phases formation energy

specific surface area

macroenergy state of activated solid

active pharmaceutical ingredient

area under curve

constant for the milled material

crystallinity of the API

particle diameter below which $10 \%$ of the sample volume exists

particle diameter below which $50 \%$ of the sample volume exists

particle diameter below which $90 \%$ of the sample volume exists

milling energy

a solid-state meloxicam containing product produced by fluidization

hydroxypropyl cellulose

high pressure homogenization

hydroxypropyl methylcellulose

the amount of a material that is sufficient to kill 50 percent of the treated population.

a solid-state meloxicam containing product produced by lyophilization

meloxicam

meloxicam dispersion

meloxicam-poly(vinyl alcohol) physical mixture

micro-sized raw meloxicam

milled nano-sized meloxicam

constant for mill

non-steroidal anti-inflammatory drug

poly(ethylene glycol)

physical mixture

poly(ethylene)-poly(propylene glycol) 
PS

PSD

PVA

PVP

rawMel

SDS

SLS

SSA

XRPD

$\mathrm{ZrO} 2$ particle size

particle size distribution

poly(vinyl alcohol)

polyvinylpirrolidone

untreated raw meloxicam

sodium dodecylsulfate

sodium lauryl sulphate

specific surface area

X-ray powder diffraction

zirconium-oxide 


\section{INTRODUCTION}

Particle design techniques are widely used to modify the physicochemical and biopharmaceutical properties of APIs (Maghsoodi et al., 2008). Particle engineering techniques that control the crystal size distribution and morphology and make use of different additives can offer improvements as concerns the solubility, rate of dissolution and permeability of poorly water-soluble drugs and can open up new, alternative administration routes as well (Pomázi et al., 2011, Ghosh et al., 2012, Maggi et al., 2013). From the early 2000's synthesis of poorly water-soluble drugs has been rapidly increased, therefore the particle size reduction especially the nanonization (particle size $<1000 \mathrm{~nm}$ ) may be the solution to reach rapid saturation concentration and better bioavailability of these drugs.

The nanonization techniques can be classified as bottom-up and top-down techniques. During the bottom-up processes nanoparticles are built from dissolved agent. Top-down processes comprise disintegration techniques, where the raw material is broken down via mechanical forces. Milling belongs among top-down methods. Due to the reduced particle size, the increased specific surface area and the surface charge of the particles can be occurred particle aggregation. To prevent the particle aggregation, the addition of stabilizing agents (polymers, surfactants) is needed (Ujhelyi et al., 2012).

New approach in a wet milling process is the combined techniques, where the planetary ball mill is combined with pearl milling technology to reach nanosize range. Planetary ball milling is mainly used in laboratory-scale research work. Pearl milling is a commonly used milling process for industrial purposes. Separately, both techniques are well known but we do not yet know enough about a combinative technique as optimization of process parameters, testing for robustness and selection of additives (polymer, surfactant).

Present $\mathrm{PhD}$ thesis shows new results on this topic, which may help nanonization of active ingredients, stabilization of nanoparticles without surfactant, and improved bioavailability of the final product. 


\section{THEORETICAL BACKGROUND}

2.1. Strategies to increase the dissolution/solubility of poorly water-soluble active agent

A significant and increasing percentage of the synthetized small molecule APIs are poorly water soluble (Merisko-Liversidge et al., 2011, Junghanns et al., 2008). Consequently, the liberation, the absorption, hence the bioavailability of these drugs is not satisfying (Liu et al., 2011).

The most important available routes to optimize the dissolution characteristics or/and the solubility properties of the APIs are the followings:

- particle size reduction (most commonly used route) (Khadka et al., 2014),

- amorphization - breakdown of the crystal structure (Jójárt-Laczkovich et al., 2016),

- polymorphism - developing a new crystal structure of the API to achieve higher solubility (Bhatia et al., 2018),

- salt forming - in the case of active substances which tend to form salt, this method can be used to enhance the water solubility (Horváth et al., 2016).

Particle size reduction techniques are widely used to produce micro- or nanoparticles in order to increase the specific surface area thereby improve the dissolution characteristics of poorly soluble drugs. According to the Noyes-Whitney equation, the dissolution rate is directly proportional to the specific surface area, therefore improved dissolution rate, higher saturated concentration and better bioavailability of the API can be achieved (Shegokar et al., 2010, Dokoumetzidis et al., 2006).

The size reduction techniques can be also classified as bottom-up and top-down techniques. During the bottom-up processes micro- or nanoparticles are built from dissolved agent with gas anti-solvent recrystallization, aerosol solvent extraction system (Yim et al., 2014f), atomized rapid injection for solvent extraction, rapid expansion of supercritical solution (Chan et al., 2011) and depressurization of an expanded liquid organic solution (Ventosa et al., 2001). Due to the complexity of these methods, as per our knowledge there are no pharmaceutical products on the market based on this technology (Shegokar et al., 2010, Finke et al., 2017).

Top-down processes comprise disintegration techniques, where the raw material is broken down via mechanical forces. The most known top-down processes: dry (Suryanarayana et al., 
2001) and wet ball milling (Peltonen et al., 2010), jet milling (Moorthi et al., 2013), high pressure homogenization (Müller et al., 2006) and high-energy sonication (Bartos et al., 2015).

\subsection{Milling}

Milling is a commonly applied technique to produce micro- or nanosized drug crystals. The main effect of the process is the comminution of particles, which results in changes in physicochemical properties of the ground material. Due to the mechanical activation, the initial structure of the material usually becomes disordered, amorphization or generation of other metastable forms can be registered (Baláž, 2008). One possible description for characterizing the macro-energy state of activated solid $(\Delta \mathrm{HT})$ equals to the sum of the energy of microstates (Pourghahramani, 2007) calculated according to the following equation:

$$
\Delta \mathrm{HT}=\Delta \mathrm{Hd}+\Delta \mathrm{HS}+\Delta \mathrm{HA}+\Delta \mathrm{HP}
$$

where $\Delta \mathrm{Hd}$ is the relative lattice distortion (dislocation density), $\Delta \mathrm{HS}$ is the specific surface area (grain boundary) and $\Delta \mathrm{HA}$ is the formation of amorphous material (Heegn, 1979) and $\Delta \mathrm{HP}$ is the formation of new phases. There are numerous types of milling techniques; dry and wet milling can be distinguished (Dahiya, 2017):

- Dry milling - the conventional method of particle size reduction.

- Wet milling - in this method, a sufficiently concentrated dispersion of drug particles in an aqueous or non-aqueous liquid medium is treated. Increased mill capacity, lower energy consumption, and easier handling of materials can be perceived as advantages of the process. However, it must be said, in the course of the milling process, increased wear of the milling medium and corrosion can occur, and the liquid agent is needed as a milling medium due to the properties of the milled materials: high residual moisture content, high potency of degradation in dry milling conditions.

\subsubsection{Dry milling}

Dry milling techniques are usually used for micronization and nanonization (Naik et al., 2015, Liu et al., 2014). Criteria of the dry milling nanonization are the use of an additive and a long milling time, furthermore, during the nanonization changes may occur in the physicochemical structure of the drug (Kürti et al., 2011). Cutter mill, roller mill and planetary ball mill can be mentioned, as machines for dry milling processes. 
The general milling law is defined by Walker et al. (Perry, 1963):

$$
d E=-\mathrm{C} \frac{d x}{x^{n}}
$$

where $E$ is grinding energy per unit mass, $\mathrm{x}$ is particle size and $\mathrm{C}$ and $\mathrm{n}$ are constants for a given material and mill. It is impossible to produce a differential decrease in particle size by grinding because mass is conserved, so that a finite quantity of small material is produced in addition to the particle of near original size. In this form, the equation is absurd (Perry, 1963).

Three sub-definitions are commonly used to determine the energy consumption of the milling: Rittinger's, Bond's and Kick's and Kirpicsov's law. In case of micronization, the Rittinger theory describes the PS reduction produced relative to the energy (E) input of milling: the new surface area generated is directly proportional to the E required for the PS reduction. As the surface area of a quantity of particles of uniform diameter $\mathrm{x}$ is proportional to $1 / x$, the E required for PS reduction is therefore also proportional (Austin, 1973):

$$
d E=C\left(\frac{1}{x_{1}}\right)-\left(\frac{1}{x_{2}}\right)
$$

In dry co-milling (the poorly water-soluble API is milled with water-soluble additives for preparation of dry nanodispersions) PVP, SDS, PEG, HPMC, and cyclodextrin derivatives are used as additives (Dahiya, 2017).

In case of milling hygroscopic or heterogenic materials (during the milling process these materials tend to stick) and nanonization (due to the high SSA and decreased particle size, instabilities can be occurred), additives need to be added. Ethylene-glycol, Aerosil ${ }^{\circledR}$ and magnesium-stearate are the most commonly used additives for dry milling processes. (Fritsch $\mathrm{GmbH}$ ). In general, many areas of industrial production avoid dry milling processes due to the higher energy demand risk of environmental contamination (Duroudier et al., 2016) and the long process time.

Planetary ball milling belongs to high energy milling methods. The working principles of the mill: during the operation the milling container rotates about its own and an external axel, so it carries out a planetary movement (Broseghini et al., 2016). According to the rotation direction, horizontal and vertical type planetary ball mill can be distinguished. In the conventional use the total kinetic energy of the milling components consists of the centrifugal force of the vial, the centrifugal force of the vial, the centrifugal force of the disk and the 
Coriolis force (Harris, 2013) (Figure 1). In terms of the energy used by the mill to achieve particle size reduction, the collision energy can be defined. The collision energy is the sum of the kinetic energy of the beads acting perpendicular to the direction of the disk rotation and the collision heat generated by the milling components and the container wall. The process is mainly used in laboratory-scale research work. It is a common technique for dry milling (Suryanarayana et al., 2001), nevertheless it is suitable for wet milling processes as well (Liu et al., 2011, Ghosh et al. 2012, George et al., 2013).

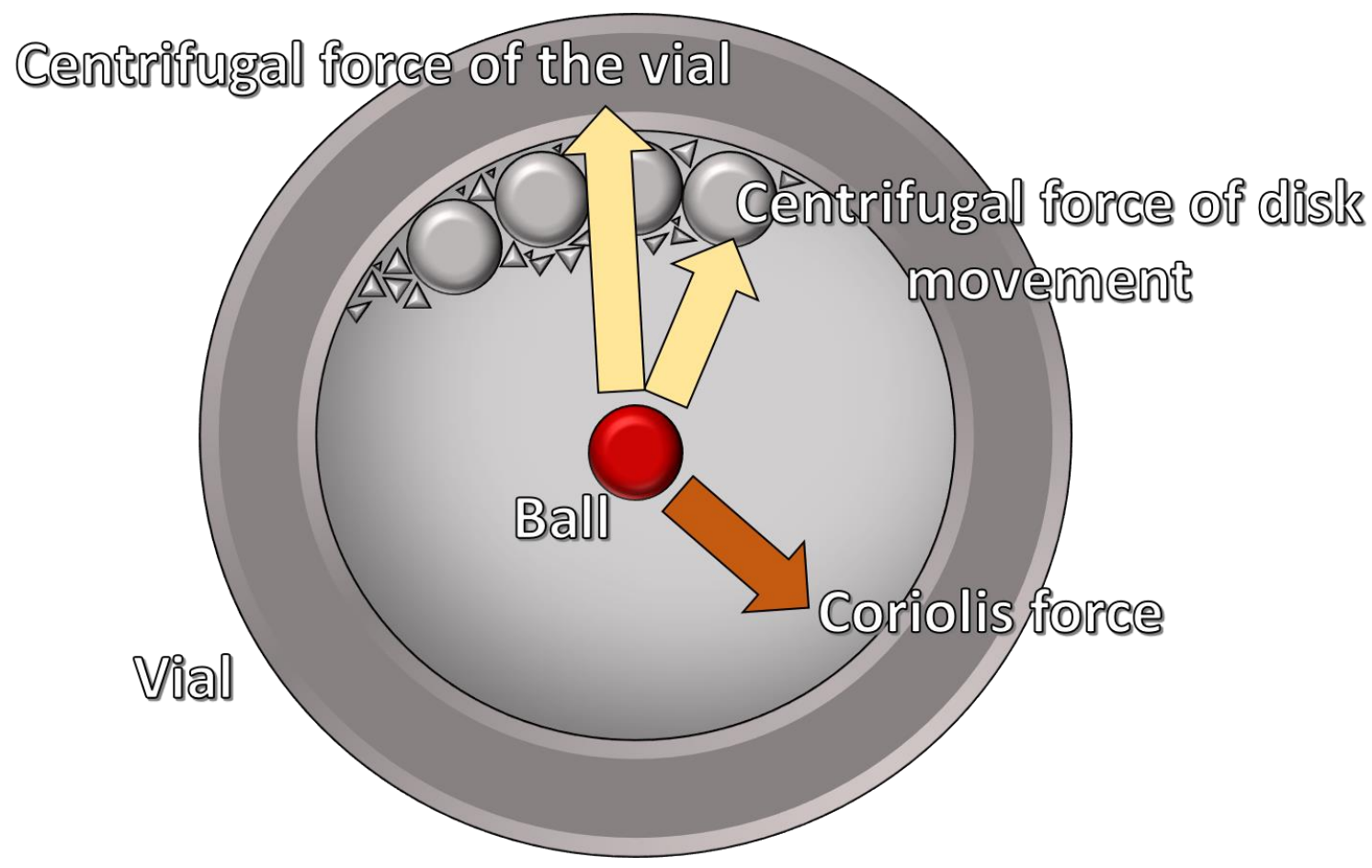

Figure 1. Energies occurring during a planetary ball milling process

\subsubsection{Wet milling}

Wet milling is a method, in which a sufficiently concentrated dispersion of drug particles in an aqueous or non-aqueous liquid medium is treated. Increased mill capacity, lower energy consumption and easier handling of materials can be perceived as advantages of the process. However, it shall be mentioned, to carry out the milling process, increased wear of the milling medium and corrosion can occur, and API instability can present difficulties. 
Wet milling is applicable for micronization (Pomázi et al., 2013), but is usually used for nanonization (Bilgili et al., 2006, Loh et al., 2015).

Drug pre-dispersions produced by wet milling can be formulated as capsules, granules, tablets, injectables, sprays and gels (Wang et al., 2013, Junyaprasert et al., 2015). The colloid mill, the toothed high-shear inline mixers, the cone mill, high-pressure homogenization and the pearl mill can be classified as wet milling equipments.

Media milling is a commonly used milling process in pigment-, photo- and magnet industry. Commercially, in pharmaceutical industry the media milling is exemplified by the NanoCrystal ${ }^{\circledR}$ technology from Elan Pharma International Ltd. (Dublin, Ireland) (Junghanns et al., 2008). In terms of the machine size, originally the mill was constructed for industrial purposes. The labor scale machine production due to extensive usability was launched (Figure 2). The milling is executed by a high energy rotating stirrer in the milling chamber, which induces the motion of the milling components. Micro-sized beads are used as milling media (100-500 $\mu \mathrm{m})$. The process is time-consuming and requires lot of energy (Merisko-Liversidge et al., 2011, Azad et al., 2014).

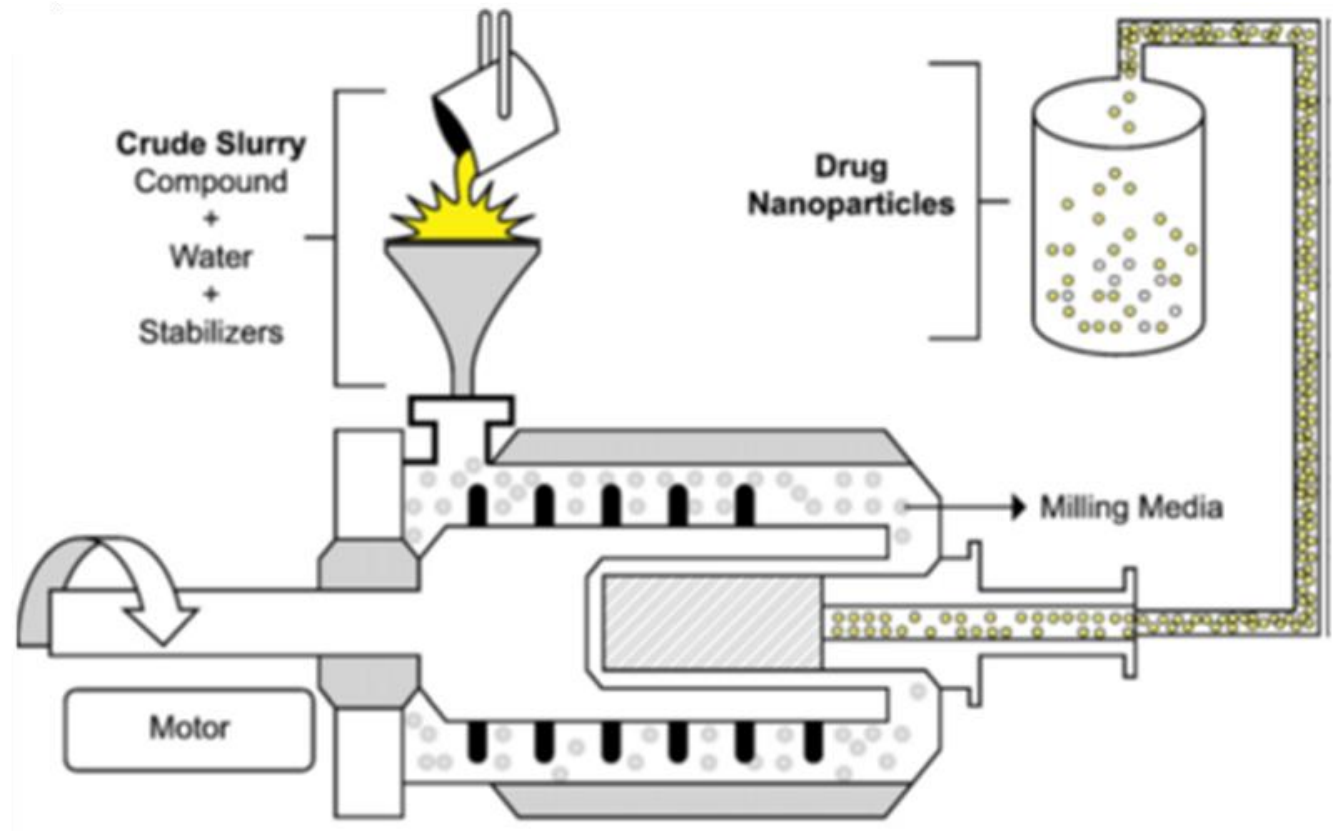

Figure 2. Schematic illustration of the wet media milling process (Loh et al. 2015).

A major drawback of media milling is the erosion of the mostly zirconia, glass or pearl milling media arising from the intensive mixing forces. Residues of the milling media may result in product contamination (Peltonen et al., 2010, Shegokar et al., 2010) leading to 
chemical destabilization of the newly formed particle surfaces and possibly affecting critical product characteristics such as PS and PSD. In case of wet milling processes, stabilizing agents are needed to prevent the physicohemical changes of the API. The main functions of a stabilizer in nanodispersions/suspensions are to wet drug particles during the milling process, and to prevent Ostwald's ripening (crystal growth in colloidal suspensions) (Kim, 2004) and agglomeration in order to yield a physically stable formulation by providing steric or ionic barriers. Different concentrations of stabilizer agents (e.g. polymers) can also influence the viscosity and the electrokinetic property of the particles, according to the DLVO theory (Derjaguin et al., 1941), and thus the stability of the nanosuspension as well. Surfactants help to wet the particles and thus reduce their aggregation tendency (Table 1) (Van Eerdenbrugh et al, 2008, Merisko-Liversidge et al., 2011). In addition to the advantages of surfactants, they have the biggest disadvantage of increasing the speed/energy of motion of the milling balls during wet milling, which can lead to the degradation of the active ingredient. When used as an external surfactant to solidify the nanosuspension, its solubility-enhancing effect may be emphasized, thereby increasing the degree of crystallinity of active agent in the solid product and reducing its dissolution rate (Verma et al., 2011). It should be noted, that the presence of surfactants is also contraindicated in some cases e.g. pediatric therapy, hypersensitivity, irritation, etc.

Conventional formulations contain these excipients in common, but the new tendency is to ignore the surfactants and look for other options to stabilize the nanoparticles in the products and achieve the desired biological effect (Bhakay et al., 2014, Azad et al., 2015, Kim et al., 2018). 
Table 1. List of additives used in wet media milling

\begin{tabular}{|c|c|c|}
\hline Polymer & $\begin{array}{l}\text { Concentration } \\
(\%)\end{array}$ & Literature reference \\
\hline HPC & $2.4-19.6$ & Lee et al. (2006), Lee (2003) \\
\hline PVA & 50 & Wiedmann et al. (1997), Bartos et al. (2018) \\
\hline PVP & 30 & Liversidge et al. (1995), Ding et al. (2019) \\
\hline Surfactant & & \\
\hline Cremophor $\AA$ & 100 & Wahlstrom et al. (2007), Chiang et al. (2007) \\
\hline Poloxamer 188 & 60 & Liversidge et al. (1995), Zhang et al. (2018) \\
\hline Poloxamer 338 & 50 & $\begin{array}{l}\text { Merisko-Liversidge et al. (1996), Mouton et al. } \\
\text { (2006) }\end{array}$ \\
\hline Poloxamer 407 & 20 & Jia et al. (2002), Zheng et al. (1997) \\
\hline Poloxamine 908 & 20 & Na et al. (1999), Girdhar et al. (2018) \\
\hline $\begin{array}{l}\text { Polyglyceryl-10 } \\
\text { laurate }\end{array}$ & 16.7 & Kondo et al. (1993), \\
\hline Tyloxapol & 20 & Ostrander et al. (1999) \\
\hline SLS & 0.15 & Vergote et al. (2001) \\
\hline Polysorbate 80 & 1 & $\begin{array}{l}\text { Merisko-Liversidge et al. (1996), Sievert et al. } \\
\text { (2018) }\end{array}$ \\
\hline $\begin{array}{l}\text { Sorbitan } \\
\text { monooleate }\end{array}$ & 1 & Merisko-Liversidge et al. (1996) \\
\hline
\end{tabular}


Crystalline state is one of the most important parameters affecting drug stability, dissolution extent, and efficacy. The high energy wet milling techniques tend to create a partially amorphous active agent. The high energy amorphous particles are unstable, especially in the presence of crystalline particles, and inclined to convert to low energy crystalline state over time. The saturation solubility between amorphous and crystalline nanoparticles is different, therefore the diffusion process will be similar to Oswald's ripening, leading to a rapid conversion of amorphous nanoparticles to crystalline state (Wu et al., 2011).

\subsubsection{Combined wet milling process}

The original meaning of the term "combined milling process" is the combination of two consecutive individual milling processes. Recently a group of combinative nanonization technologies were developed. There are five known groups: NANOEDGE ${ }^{\mathrm{R}}$ (microprecipitation followed by HPH), microprecipitation immediately followed by HPH, spray-drying followed by HPH, lyophilization followed by $\mathrm{HPH}$, and the combinative technology (media milling followed by HPH) (Shegokar and Muller, 2010).

The combined wet milling process used in this research work is a fusion of two basic milling techniques, in both cases the milling is performed in presence of grinding media (beads). In the Retsch method recommendation list, combination of the planetary ball mill and the grinding media of the pearl mill is preferred as a novel milling technique in order to prepare pre-dispersions with nanoparticle size of the API (Retsch GmbH, Haan, Germany). This combined milling technique unites the advantageous properties of the basic milling processes:

- Particle size reduction with high energy transfer in labor-scale conditions, produced by the planetary ball mill.

- The micro-sized beads of the wet stirred media milling give a higher reduction rate according to the basically used millimeter-scale beads - the particle size reduction rate is inversely proportional to the size of the bead size (Gao et al., 1995).

The milling is followed by a bead-elimination process: the concentrated milled dispersions are filtered by a sieve with $150 \mu \mathrm{m}$ mesh size in order to remove the pearls. For the washing of the pearls, purified water is used. Due to the dilution, the particle-stabilizing effect of the applied additive will not be satisfactory. 
The robustness testing of the milling process is a necessary method to discover the limits of the process parameters variabilities while the product quality can be maintained (Nalluri et al., 2010). During the optimization process, beside the optimal process parameters, the range of parameters can also be determined, and as a result, a nanosuspension can be provided which as an intermediate product, with further modification or stabilization routes can be transformed into a liquid, semi-solid or solid state drug delivery systems (Bartos et al., 2019).

2.3. Transformation of nanosuspension as an intermediate product into solid state product (fluidization, lyophilization)

Nanosuspensions can be defined as colloidal dispersions of nanosized drug particles $(<500$ $\mathrm{nm}$ ) that are produced by different nanonization processes and stabilized by various excipients (Müller et al., 1998). Nanocrystals also enhance adhesiveness to the gastrointestinal mucosa, resulting in prolonged gastrointestinal residence and, thus, increased uptake via the gastrointestinal tract (Malamatari et al., 2018).

Despite the advantages of drug nanoparticles, they present various drawbacks including sedimentation, particle agglomeration or crystal growth. During the wet nanonization, due to the reduced particle size, the increased specific surface area, the surface charge of the particles and the high particle mobility, particle aggregation can be occurred. To prevent the particle aggregation, further stabilization is needed (Peltonen et al., 2010).

Nanosuspensions can be applied as final liquid dosage forms using further different excipients (viscosity enhancer, flavoring, preservative agents, etc.), however, their stabilization is a major challenge (Wang et al. 2013, Zheng et al., 2013). It is well known that, despite the stabilization, nanosuspensions have a short expiration time, and there are patients who do not prefer this form or the presence of a surfactant. One way to overcome the instability and surfactant problem is to design solid dispersion (Nagy et al., 2012) produced by spray drying, spray freeze drying and freeze drying (lyophilization) (Fülöp et al. 2018) It is well known that the dry nanosuspensions can cause difficulty in hydration and redispersibility (Kim et al., 2018, Van Eerdenbourgh et al., 2008). Other processes for transforming a nanosuspension into solid-state forms (tablets, capsules) are very different: deposition as coatings, incorporation in granules and pellets and the 3D printing technologies (Prasad et al., 2016).

Spray drying and lyophilization (freeze drying) are the commonly used techniques for the solidification of nanosuspension because of their easy application and industrial acceptability 
(Colombo et al., 2017) The powders produced by these processes often suffer from poor flowability and high hygroscopicity, therefore other technologies are applied to transform nanosuspensions into oral dosage forms as tablets and capsules (Kayaert et al., 2011).

Layering of nanosuspension onto the surface of granules, pellets, sugar beads, etc. using a fluidization technique is used as an alternative method for solidification of nanosuspension (Möschwitzer et al, 2006, Kayaert et al., 2011, He et al., 2013, A. Azad et al., 2014, Mittal, 2017). The advantage of this process that may be used various additives in order to achieve the desired purpose, e.g. i) different polymers stabilize the nanosuspension, and act as a coating materials resulted in fast dissolution rate, ii) surfactants prevent the aggregation of nanoparticle and can modify the drug release (Vergote et al., 2001, Bose et al., 2012). In any case, the fluidization technique also provides an opportunity to stabilize the broken structure of the ground crystals in the nanosuspension by using crystallization inhibitors (JójártLaczkovich et al., 2011).

The water solubility of NSAIDs is poor or very poor in general but increasing $\mathrm{pH}$ results in higher solubility (Szabó-Révész, 2018). Mel, a member of the oxicam family of NSAIDs, as a moderately selective cyclooxygenase (COX-2) inhibitor can have a role in acute pain therapy but a basic requirement is rapid absorption through the gastric mucosa. Mel has a weak acidic character with $\mathrm{pKa}$ of 3.43 , therefore its solubility in gastric juice $(\mathrm{pH}=1.2)$ is very poor but its $\log \mathrm{P}_{\text {app }}$ is $2.43(\mathrm{pH}=2.0)$, which predestines the fast absorption from the stomach (Simmons et al., 2004, Bartos et al., 2016, Szabó-Révész, 2018). Since the solubility of Mel is very poor in gastric juice, the preparation of a nanosuspension with a fast dissolution rate may be a solution.

PVA is a polymer, which is frequently used as a stabilizer, coating the particles and promoting their separation from each other (Bartos et al., 2015).

The acute oral toxicity of PVA is very low, with LD50s in the range of $15-20 \mathrm{~g} / \mathrm{kg}$. Orally administered PVA is very poorly absorbed from the gastrointestinal tract and it does not accumulate in the body when it is administered orally. The PVA is not mutagenic or clastogenic. It can be concluded, that the orally distributed PVA is nontoxic (De Merlis et al., 2003). 


\section{AIMS}

The aim of this work was to study the wet milling process, where the planetary ball mill was combined with pearl milling technology and optimize the process parameters and predict the robustness of the process and design the amount of the additive (PVA) in order to produce Mel containing nanosuspension. PVA as a protective polymer had a dual function, partly to increase the efficiency of milling (without any pre-milling procedure, surfactant free samples) and partly to stabilize the Mel containing nanosuspension as an intermediate product. Furthermore, the optimized Mel formulations were tested on the cell culture model of intestinal epithelium.

The purpose of the work was further to produce a surfactant-free product by solidifying of Mel containing nanosuspension. Critical product parameters were considered to be the particle size distribution of the $\operatorname{drug}(\mathrm{d}(0.9)<500 \mathrm{~nm})$, stabilization of the degree of crystallinity altered during milling, and enhancement of the bioavailability of the solid product with fast absorption from the stomach for rapid analgesia. The transformation of the nanosuspension was done by fluidization and lyophilization.

The main steps in the experiments were as follows:

i. Optimization of the critical process parameters of the combined wet milling (ratio of predispersion and pearls, milling time and rotation speed) in order to produce Mel containing nanosuspension as an intermediate product without any pre-treating procedure and surfactant (nanoMel).

ii. Investigation of the influence of the PVA amount on the milling effectiveness and the PSD and crystallinity of the Mel in the ground products which does not contain any additional excipients, thus surfactant.

iii. Perform in vitro dissolution test and cell culture studies (cell viability and permeability) to control the amount of PVA.

iv. Testing the robustness of combined wet milling process to determine the interval of the Mel amount and to predict the degree of crystallinity of the milled products.

v. Transformation of the surfactant-free nanoMel sample into solid-state products by fluidization and lyophilization and investigation of the product stability (particle size, crystallinity), and in vitro release of Mel 
vi. Bioavailability of the products was studied by in vivo animal tests to justify applicability of the surfactant-free samples containing nanonized Mel.

\section{MATERIALS AND METHODS}

\subsection{Materials}

The characteristics of $\mathrm{Mel}$ and the used additives are shown in Table 2. All other reagents used during the work were purchased from Sigma-Aldrich, Ltd. (Budapest, Hungary)

Table 2. Properties of the materials used in the research work

\begin{tabular}{|c|c|c|c|c|}
\hline & Mel & PVA & $\mathrm{MCC}$ & Trehalose \\
\hline Chemical & & & & ${ }^{\mathrm{OH}}{ }^{\mathrm{HO}} \mathrm{OH}^{\mathrm{H}}$ \\
\hline $\begin{array}{l}\text { Chemical } \\
\text { name }\end{array}$ & $\begin{array}{l}\text { 4-hydroxy-2- } \\
\text { methyl- } N \text {-(5- } \\
\text { methyl-2- } \\
\text { thiazolyl)-2H-1,2 } \\
\text { benzothiazine-3- } \\
\text { carboxamide-1,1- } \\
\text { dioxide }\end{array}$ & $\begin{array}{l}\text { Poly(vinyl } \\
\text { alcohol) }\end{array}$ & $\begin{array}{l}\text { Microcrystalline } \\
\text { cellulose }\end{array}$ & $\begin{array}{l}\text { Alpha-D- } \\
\text { trehalose- } \\
\text { dihydrate }\end{array}$ \\
\hline Manufacturer & $\begin{array}{l}\text { EGIS Ltd. } \\
\text { (Budapest, } \\
\text { Hungary) }\end{array}$ & $\begin{array}{l}\text { Sigma Aldrich } \\
\text { Co. LLC, St. } \\
\text { Louis MO, USA }\end{array}$ & $\begin{array}{l}\text { FMC } \\
\text { Biopolymer, } \\
\text { Philadelphia USA }\end{array}$ & $\begin{array}{l}\text { Karl Roth GmbH } \\
\text { + Co. KG. } \\
\text { (Karlsruhe, } \\
\text { Germany) }\end{array}$ \\
\hline $\begin{array}{l}\text { Physical } \\
\text { properties }\end{array}$ & $\begin{array}{l}\text { Mw: } 351.4 \mathrm{~g} / \mathrm{mol} \\
\text { a yellow powder } \\
\text { poor solubility in } \\
\text { water }\end{array}$ & $\begin{array}{l}\text { Mw: } 44.05 \mathrm{~g} / \mathrm{mol} \\
\text { a white powder } \\
\text { soluble in water, } \\
\text { slightly soluble in } \\
\text { ethanol semi- } \\
\text { crystalline }\end{array}$ & $\begin{array}{l}\text { Mw:370.35 g/mol } \\
\text { a white powder, } \\
\text { insoluble in water }\end{array}$ & $\begin{array}{l}\text { Mw:342.3 g/mol } \\
\text { a white powder, } \\
\text { soluble in water, }\end{array}$ \\
\hline
\end{tabular}

\subsection{Methods}

\subsubsection{Optimization of combined wet-milling process}

The samples were milled with the steel jar with $50 \mathrm{ml}$ volume of the Retsch PM 100 planetary ball mill (Retsch PM $100 \mathrm{MA}$, Retsch GmbH, Germany) combined with $0.3 \mathrm{~mm} \mathrm{ZrO}_{2}$ beads 
as the grinding media. The concentrated $(10 \% \mathrm{w} / \mathrm{w})$ pre-dispersions $(2 \mathrm{~g}$ of Mel suspended in $18 \mathrm{~g}$ of aqueous dispersant medium containing PVA) were added to the $\mathrm{ZrO}_{2}$ beads. In the first step, the effect of different amount of $\mathrm{ZrO}_{2}$ pearls on the particle size reduction was investigated. The ratio of the amount of pre-dispersion and pearls (w/w) was 1:0.5, 1:1, 1:2 and 1:4; and the milling times were 10, 30 and $50 \mathrm{~min}$. In these cases, the concentration of PVA solution was $2.5 \%$ (w/w), and the rotation speed was $400 \mathrm{rpm}$. In the second step, design and analysis of experiments with three levels were used to optimize the milling time $(10,30$ and $50 \mathrm{~min})$ and the rotation speed $(200,350$ and $500 \mathrm{rpm})$ as independent variables. The amount of the pearls was chosen on the basis of the optimization study. The temperature of the samples was measured immediately after milling. This value did not exceed $39^{\circ} \mathrm{C}$.

\subsubsection{Optimization of PVA concentration}

After optimizing the process parameters, the determination of the adequate PVA concentration was executed. Various amounts of PVA (2.5-7.5\%) were applied to prepare the concentrated pre-dispersions. The concentration of the PVA solutions was increased with a half percent in the range. Mel content was $10 \%(\mathrm{w} / \mathrm{w})$ and the optimized process parameters were used during the milling. The degree of particle size reduction and particle size distribution were the main factors for the optimization of the PVA concentration (Figure 3).

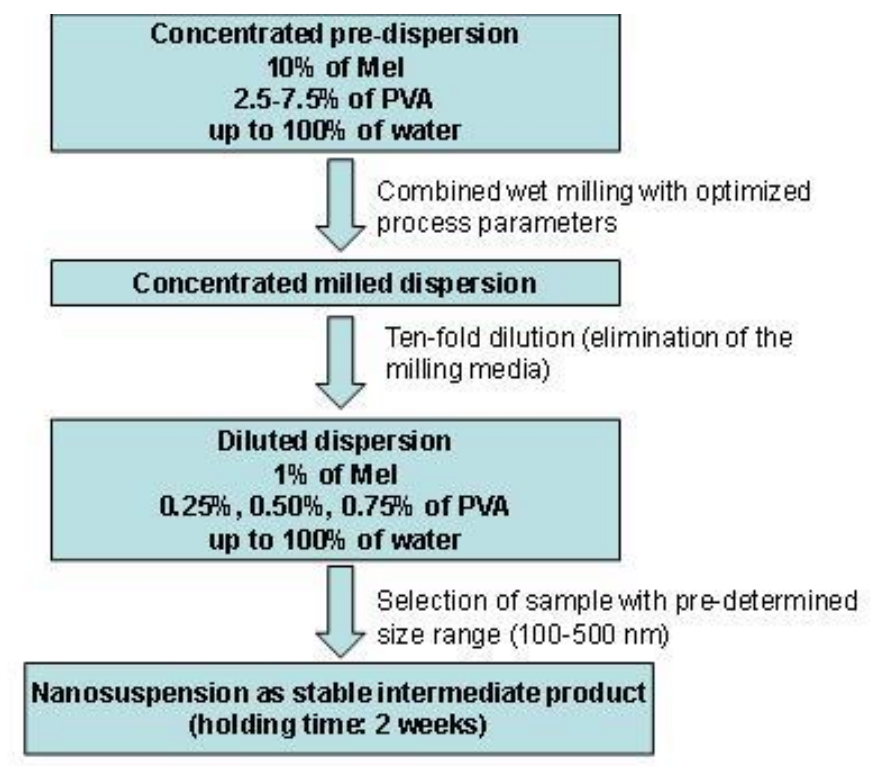

Figure 3. Protocol of sample preparation in optimization of PVA content

Each milled product was filtered by a sieve with $150 \mu \mathrm{m}$ mesh size in order to remove the pearls. For the washing of the pearls, $180 \mathrm{~g}$ of purified water was used. In all cases, the milled 
dispersions were ten-fold diluted products with $1 \%$ of Mel (w/w) content. The samples were selected on the basis of the particle size range $(100-500 \mathrm{~nm})$ and the holding time (2 weeks).

\subsubsection{Robustness determination of the process}

Eight samples were prepared from 0.5 to $4.0 \mathrm{~g}$ of Mel with $0.5 \mathrm{~g}$ increment per sample. As the stabilizing agent, 5\% of PVA aqueous solution was added up to $20.0 \mathrm{~g}$ to each sample, which was selected on the basis of our previous experiments. The Mel and PVA concentrations of the samples are shown in Table 3.

Table 3. Mel and PVA concentrations in the samples

\begin{tabular}{|l|l|l|}
\hline Sample name & $\begin{array}{l}\text { Mel\% } \\
(\mathrm{w} / \mathrm{w})\end{array}$ & $\begin{array}{l}\text { PVA\% } \\
(\mathrm{w} / \mathrm{w})\end{array}$ \\
\hline Mel_0.5g & 2.50 & 5.42 \\
\hline Mel_1.0g & 5.00 & 5.28 \\
\hline Mel_1.5g & 7.50 & 5.14 \\
\hline Mel_2.0g* & 10.00 & 5.00 \\
\hline Mel_2.5g & 12.50 & 4.86 \\
\hline Mel_3.0g & 15.00 & 4.72 \\
\hline Mel_3.5g & 17.50 & 4.58 \\
\hline Mel_4.0g & 20.00 & 4.44 \\
\hline
\end{tabular}

*optimized sample

\subsubsection{Transformation of nanosuspension into solid compositions}

\subsubsection{Fluidization (fluidMel)}

The Avicel PH 101 as the carrier material was used in a Strea-1 (Niro Aeromatic, Bubendorf, Switzerland) fluid bed chamber. A batch size of $100 \mathrm{~g}$ was used. The powder was inserted and fluidized in the preheated chamber for a period of $10 \mathrm{~min}$ and at constant air velocity of $2.5 \mathrm{~m} / \mathrm{s}$. NanoMel as liquid dispersion was implemented using a peristaltic pump (Roto Consulta Ebikon/Luzern, Schweiz), the applied pump speed was $9 \mathrm{rpm}$. The process took 50 minutes, the inlet temperature was $55^{\circ} \mathrm{C}$, the outlet temperature was $38^{\circ} \mathrm{C}$. One batch of nanoMel sample (200g) was transferred onto the surface of the material, thus the fluidized solid-state product contained 2g of Mel, 1g of PVA and 100g of Avicel 101 (Table 4). 
Table 4. Composition of the investigated samples

\begin{tabular}{|l|l|l|l|l|l|}
\hline Sample & Mel & PVA & Water & MCC & Trehalose \\
& $(\%)$ & $(\%)$ & $(\%)$ & $(\%)$ & $(\%)$ \\
\hline fluidMel $^{\mathrm{a}}$ & 1.94 & 0.97 & - & 97.09 & - \\
\hline lyoMel $^{\mathrm{b}}$ & 15.38 & 7.70 & - & - & 76.92 \\
\hline
\end{tabular}

\subsubsection{Lyophilization (lyoMel)}

Freeze-drying was performed in Scanvac CoolSafe 100-9 Pro type equipment (LaboGene ApS, Lynge, Denmark) equipped with a 3-shelf sample holder unit, recessed into the drying chamber. In each cuvette $750 \mathrm{mg}$ of diluted milled suspension was filled (7.5 mg Mel content in every cuvette, the unit dose of Mel). As a cake-forming additive, $37.5 \mathrm{mg}$ of $(5.0 \%)$ trehalose was dissolved in the nanosuspensions. The components of the final product can be seen in Table 4. The process was controlled by a computer program (Scanlaf CTS16a02), the temperature and pressure values were recorded continuously. The whole process took 71 hours and 52 minutes. The initial temperature was $25{ }^{\circ} \mathrm{C}$. During the freezing period, after 18 hours and 34 minutes, the sample temperature was decreased to $-40{ }^{\circ} \mathrm{C}$. The subsequent drying process was conducted at 0.013 mbar air pressure for 50 hours and 50 minutes, the temperature increased from $-40{ }^{\circ} \mathrm{C}$ to $-7{ }^{\circ} \mathrm{C}$. Finally, the secondary drying session took 2 hours and 28 minutes. The final temperature of the dried products was $30^{\circ} \mathrm{C}$.

\subsubsection{Investigation of the samples}

\subsubsection{Particle size distribution measurements}

The investigations on the particle size of raw Mel and nanoMel via laser diffraction were executed (Malvern Mastersizer S 2000, Malvern Instruments Ltd, Worcestershire, UK) with the following parameters: 300RF lens; small volume dispersion unit (1,000 rpm); refractive index for Mel: 1.720; and refractive index for dispersion medium: 1.330. During the measurements, distilled water was used as a dispersant, and obscuration was in the range of $11 \%-16 \%$ for all measurements. In both cases, the particle size distributions were qualified by $\mathrm{d}(0.1), \mathrm{d}(0.5)$, and $\mathrm{d}(0.9)$. 
In the case of the solid-state products (fluidMel and lyoMel), the particle size of Mel was determined by using Scanning Electron Microscopy (SEM) images (Hitachi S4700, Hitachi Scientific Ltd., Tokyo, Japan). The size of the particles was calculated by ImageJ software for Windows (Phase GmbH, Lübeck, Germany) (Abramoff et al., 2004). By specifying the unit length, which is shown in each images (depending on the magnification this is a different value), the actual particle size can easily be determined by drawing the diameter of the captured particles. A diameter of a hundred captured particles was determined in the case of two solid phase samples.

For nanoMel and lyoMel samples, the Z-average particle size and the polydispersity index (PDI) of Mel were measured using a Malvern Zeta Nano ZS (Malvern Instruments Ltd). In case of the particle size determination, Malvern DTS 1070 folded capillary cell was used. The samples were further diluted with water (25-fold) for the measurements.

\subsubsection{Image analysis (scanning electron microscopy - SEM)}

The particle morphology of milled dispersions and the solid-state samples were investigated. The diluted milled dispersions were dried in a vacuum dryer (Binder GmbH, Tuttlingen, Germany) at $40{ }^{\circ} \mathrm{C}$ in order to obtain solid products for physicochemical investigations. The shape and surface characteristics of the samples were visualized by SEM (Hitachi S4700, Hitachi Scientific Ltd., Tokyo, Japan). The samples were sputter-coated with gold-palladium under an argon atmosphere, using a gold sputter module in a high-vacuum evaporator, and the samples were examined at $10 \mathrm{kV}$ and $10 \mathrm{~mA}$. The air pressure was $1.3-13 \mathrm{MPa}$.

\subsubsection{X-ray powder diffraction analysis (XRPD)}

The crystallinity state of Mel in the samples was evaluated by XRPD. XRPD patterns were produced by a Bruker D8 Advance diffractometer (Bruker AXS GmbH, Karlsruhe, Germany) system with $\mathrm{Cu} \mathrm{K} \lambda \mathrm{I}$ radiation $(\lambda=1.5406 \AA)$. The samples were scanned at $40 \mathrm{kV}$ and 40 $\mathrm{mA}$ from 3 to $402 \theta$, at a step time of $0.1 \mathrm{~s}$ and a step size of $0.010^{\circ}$. The instrument was calibrated by using Si standard. The semi-quantitative determination of Mel crystallinity (Cryst. \%) was performed using the total area under the curve (AUC) of 3 characteristic peaks $\left(13.06^{\circ}, 14.94^{\circ}\right.$ and $\left.18.61^{\circ} 2 \Theta\right)$ of Mel. The AUC value of the peak of raw material without milling (rawMel) and the dried milled dispersions (MelD) was calculated and compared according the following formula (Rafiq et al., 2015) 


$$
\text { Cryst. } \%=\frac{A U C M e l D}{A U C r a w M e l} * 100
$$

\subsubsection{Stability test}

The products (fluidMel and lyoMel) were stored in a well-closed container, at room temperature $\left(23 \pm 2{ }^{\circ} \mathrm{C}, 45 \pm 5 \% \mathrm{RH}\right)$ for 6 months. The crystallinity of Mel was investigated compared to freshly measured products.

\subsubsection{Differential scanning calorimetry (DSC)}

DSC measurements were carried out with a Mettler Toledo DSC 821e thermal analysis system with the $\mathrm{STAR}^{\mathrm{e}}$ thermal analysis software V9.0 (Mettler Inc. Schwerzenbach, Switzerland). Approx. 2-5 mg of pure Mel and PVA as well as dried samples were examined in the temperature range of $25-270^{\circ} \mathrm{C}$. The heating rate was $10^{\circ} \mathrm{C} / \mathrm{min}$ in the presence of argon as a carrier gas with a flow rate of $100 \mathrm{ml} / \mathrm{min}$.

\subsubsection{Raman spectroscopy}

For the investigation of Mel degradation as a function of the pearl amount and milling time in the dispersion, Raman spectra were acquired with a Thermo Fisher DXR Dispersive Raman (Thermo Fisher Sco. Inc., Waltham, MA, USA) equipped with a CCD camera and a diode laser operating at a wavelength of $532 \mathrm{~nm}$. Raman measurements were carried out with a laser power of 4 and $8 \mathrm{~mW}$ at $25 \mu \mathrm{m}$ slit aperture size on a $2 \mu \mathrm{m}$ spot size. The spectra of the individual substances as Mel and PVA were collected using a 2-sec exposure time, a total of 48 scanning in the spectral range of $3300-200 \mathrm{~cm}^{-1}$ with cosmic ray and fluorescence corrections.

\subsubsection{Zeta potential measurements}

The zeta potential of the dispersions and the lyophilized product was measured via Malvern Zeta Nano ZS (Malvern Instruments Ltd, Worcestershire, UK). The refractive index was 1.720. For the zeta potential determination, Malvern DTS 1070 folded capillary cell was used at $25^{\circ} \mathrm{C}$. The diluted milled dispersions were further diluted with water (25-fold) for the measurements.

\subsubsection{Rheological investigations}

To investigate the viscosity changes during the milling process, the initial and milled PVA solutions and the concentrated milled dispersions were used. Rheological measurements were 
carried out with Physica MCR101 rheometer (Anton Paar, Austria, Graz). A concentric cylinder measuring device with a diameter of $17 \mathrm{~mm}$ was used. The flow curves of the samples were determined at $25{ }^{\circ} \mathrm{C}$, where the shear rate was increased from 0.1 to $100 \mathrm{1} / \mathrm{s}$. The shearing time was $300 \mathrm{~s}$. In this paper, viscosity values were given at $100 \mathrm{1} / \mathrm{s}$ shear rate. Two parallel measurements were run.

\subsubsection{Solubility testing of $\mathrm{Mel}$ in the pre-dispersions}

The solubility of Mel in the samples (rawMel, nanoMel, fluidMel and lyoMel) was determined. The dispersions were stirred with a magnetic stirrer at $25{ }^{\circ} \mathrm{C}$ for $24 \mathrm{~h}$ and then filtered (0.1 $\mu \mathrm{m}$, FilterBio PES Syringe Filter) (Labex Ltd., Budapest, Hungary), and the dissolved drug content was analyzed spectrophotometrically (Unicam UV/VIS) (Thermo Fisher Scientific Inc., Waltham, MA, USA) at $364 \mathrm{~nm}$ wavelength $(\mathrm{n}=3)$.

\subsubsection{Drug content determination}

The Mel content of the samples was controlled in the following way. The unit dose of the products with $0.75 \mathrm{mg}$ of theoretical Mel was dissolved in $100 \mathrm{~mL}$ of phosphate buffer $\mathrm{pH} 7.4$ \pm 0.1 . The sample was stirred with a magnetic stirrer at $25{ }^{\circ} \mathrm{C}$ for $24 \mathrm{~h}$ and then filtered $(0.1$ $\mu \mathrm{m}$, FilterBio PES Syringe Filter) (Labex Ltd., Budapest, Hungary), and the concentration of the dissolved Mel was analyzed spectrophotometrically (Unicam UV/VIS) (Thermo Fisher Scientific Inc., Waltham, MA, USA) at $364 \mathrm{~nm}$ wavelength. The investigations were repeated three times.

\subsubsection{In vitro studies}

\subsubsection{In vitro dissolution studies of Mel}

To determine the dissolution extent of Mel from dispersions, the paddle method (USP dissolution apparatus, type II Pharma Test, Heinburg, Germany) was used. $750 \mathrm{mg}$ of the dispersion with $7.5 \mathrm{mg}$ of Mel (therapeutic dose) was filled into hard gelatin capsules within 5 sec and put promptly into the medium. The medium was $900 \mathrm{ml}$ of artificial gastric fluid at $\mathrm{pH} 1.2 \pm 0.1$ and intestinal fluid $(\mathrm{pH} 6.8 \pm 0.1)$. The paddle was rotated at $100 \mathrm{rpm}$ and sampling was performed up to $60 \mathrm{~min}$. The Mel contents of the samples were determined by spectrophotometer (ATI-UNICAM UV/VIS Spectrophotometer, Cambridge, UK) at $362 \mathrm{~nm}$ (gastric juice) and $364 \mathrm{~nm}$ (enteric fluid). The number of parallels was three. 


\subsubsection{In vitro cell culture studies}

\subsection{Human Caco-2 intestinal epithelial cell line}

Caco-2 intestinal epithelial cell line was purchased from ATCC (cat.no. HTB-37) and used until passage 60 for the experiments. The cells were grown in Dulbecco's Modified Eagle's Medium (Gibco, Life Technologies, Carlsbad, California, USA) supplemented with $10 \%$ fetal bovine serum (Pan-Biotech $\mathrm{GmbH}$, Aidenbach, Germany) and $50 \mu \mathrm{g} / \mathrm{ml}$ gentamycin in a humidified incubator with $5 \% \mathrm{CO} 2$ at $37^{\circ} \mathrm{C}$. All plastic surfaces were coated with $0.05 \%$ rat tail collagen in sterile distilled water before cell seeding in culture dishes and the medium was changed every 2 days. The stock solutions for cell culture experiments were the following: Mel, $100 \mathrm{mg} / \mathrm{ml}$; PVA, 5\% (w/w) and three products containing $100 \mathrm{mg} / \mathrm{ml} \mathrm{Mel} \mathrm{with} \mathrm{various}$ amounts of PVA $(2.5,5$ or $7.5 \%)$. The working solutions were diluted in cell culture medium or Ringer-Hepes buffer and contained $1 \mathrm{mg} / \mathrm{ml}$ of meloxicam for the Mel and formulation groups. The final concentration of PVA was $0.025,0.05$ and $0.075 \%(w / w)$.

\subsection{Cell viability measurement by impedance}

Impedance was measured at $10 \mathrm{kHz}$ by RTCA SP instrument (RTCA-SP instrument, ACEA Biosciences, San Diego, CA, USA). This method is label-free, non-invasive and follows cell adherence, growth, number and viability real time. We have successfully tested the cellular effects of peptides and pharmaceutical excipients by impedance kinetics.28,29,30 For background measurements $50 \mu \mathrm{l}$ cell culture medium was added to the wells, then cells were seeded at a density of $6 \times 103$ cells/well to 96-well plate with gold electrodes (E-plate 96, ACEA Biosciences) coated with collagen. Cells were cultured for 5 days in $\mathrm{CO}_{2}$ incubator at $37^{\circ} \mathrm{C}$ and monitored every 10 minutes until the end of experiments. At the beginning of plateau phase of growth cells were treated with Mel, PVA and Mel+PVA samples diluted in cell culture medium and the effects were followed for 8 hours. Triton X-100 detergent (1 $\mathrm{mg} / \mathrm{ml}$ ) was used as a reference compound to induce cell toxicity. Cell index was defined as $\mathrm{Rn}-\mathrm{Rb}$ at each time point of measurement, where $\mathrm{Rn}$ is the cell-electrode impedance of the well when it contains cells and $\mathrm{Rb}$ is the background impedance of the well with the medium alone.

\subsection{Permeability study on cell culture model}

Transepithelial electrical resistance (TEER) reflects the tightness of the intercellular junctions closing the paracellular cleft, therefore the overall tightness of cell layers of biological 
barriers. TEER was measured every 2 day to check the barrier integrity by an EVOM voltohmmeter (World Precision Instruments, Sarasota, FL, USA) combined with STX-2 electrodes, and was expressed relative to the surface area of the monolayers as $\Omega \times \mathrm{cm}^{2}$.

Caco-2 cells were seeded onto Transwell inserts (polycarbonate membrane, $0.4 \mu \mathrm{m}$ pore size, $1.12 \mathrm{~cm}^{2}$ surface area; Corning Life Sciences, Tewksbury, Massachusetts) and cultured for three weeks (Hellinger et al., 2012, Kiss et al., 2014). The culture medium was changed and TEER was checked every second day.

For permeability experiment inserts were transferred to 12 -well plates containing $1.5 \mathrm{ml}$ Ringer-Hepes buffer in the lower (basal) compartments. In the upper (apical) compartments culture medium was replaced by $0.5 \mathrm{ml}$ buffer containing treatment solutions of Mel, PVA and Mel formulation groups for 1 hour. Permeability marker molecules fluorescein $(10 \mu \mathrm{g} / \mathrm{ml}$; Mw: $376 \mathrm{Da})$ and albumin (10 mg/ml; Mw: $65 \mathrm{kDa})$ labeled with Evans blue $(167.5 \mu \mathrm{g} / \mathrm{ml})$ were used for verifying the cell layer integrity. Treatment solutions from both compartments were collected and the Mel level was detected by Thermo Spectronic Helios Alpha UV-VIS spectrophotometer (Thermo Fisher Scientific Inc., Waltham, Massachusetts, USA).

\subsubsection{Holding time determination of Mel in the pre-dispersions}

Since the diluted dispersions are intermediate products, it was necessary to specify the "holding time" of the sample through the particle size distribution. The products were stored in sealed glass bottles at room temperature $\left(25^{\circ} \mathrm{C} \pm 1^{\circ} \mathrm{C}\right)$ for 2 weeks. The particle size distribution of the Mel in the samples was analyzed on the day of production (day 0) and after 14 days of storage.

\subsubsection{In vivo study of Mel}

Each sample contained $60 \mu \mathrm{g} / \mathrm{ml}$ of $\mathrm{Mel}$ and $30 \mu \mathrm{g} / \mathrm{ml}$ of PVA in purified water. For per os delivery, the different formulations were individually diluted and were given at a single dose of $300 \mu \mathrm{g} / \mathrm{kg}$ of Mel to male Sprague-Dawley rats (8 weeks old, 240-260 g, n =6) in a volume of $0.5 \mathrm{ml}$ by gastric gavages. All animals fasted 16 hours before the per os administration of drugs. In order to facilitate the absorption, the solid-state forms were redispersed in water immediately before administration. In a comparison study for intravenous administration, animals were treated with a $300 \mu \mathrm{g} / \mathrm{kg}$ bolus of Mel via the tail vein. 
Intravenous (IV) injection was prepared by the dilution of passable injection with a concentration of $15 \mathrm{mg} / 1.5 \mathrm{~mL}$ (Meloxicam-Zentiva, Prague, Czech Republic) to reach the final concentration $(0.15 \mathrm{mg} / \mathrm{mL})$. The ingredients of the injection were meglumine, poloxamer 188, glycine, sodium hydroxide (for $\mathrm{pH}$ adjustment), sodium chloride, glycopherol, and water for injection. Blood samples were collected from the tail vein before and at 15, 30, 60, 75, 90, 120 and 180 minutes post-dosing. The experimental protocols and animal care methods used in the present study were approved by the National Scientific Ethical Committee on Animal Experimentation (permission number IV/1247/2017).). The animals were treated in accordance with the European Communities Council Directives (2010/63/EU) and the Hungarian Act for the Protection of Animals in Research (Article 32 of Act XXVIII).

Plasma samples were collected into EDTA containing polyethylene tubes, centrifuged at $1,500 \mathrm{~g}$ for $10 \mathrm{~min}$ at $5{ }^{\circ} \mathrm{C}$. Separated plasma samples were stored at $-80{ }^{\circ} \mathrm{C}$ until extraction and analysis.

Determination of Mel from rat plasma

Preparation of plasma samples, calibration standards and quality control samples

To $90 \mu \mathrm{L}$ of plasma sample, $10 \mu \mathrm{L}$ of $0.1 \%$ aqueous formic acid and $300 \mu \mathrm{L}$ of acetonitrile containing piroxicam (internal standard at $12.5 \mathrm{ng} / \mathrm{mL}$ concentration) were added and the mixture was vortex-mixed for $60 \mathrm{~s}$. The mixture was allowed to rest for $30 \mathrm{~min}$ at $-20^{\circ} \mathrm{C}$ to support protein precipitation. Supernatant was obtained by the centrifugation of the mixture for $10 \mathrm{~min}$ at $10,000 \mathrm{~g}$ at $4{ }^{\circ} \mathrm{C}$ and $20 \mu \mathrm{L}$ was diluted with $380 \mu \mathrm{L}$ of $0.1 \%$ aqueous formic acid. Finally, $5 \mu \mathrm{L}$ was injected into the LC-MS/MS system for analysis.

Rat plasma calibration standards of meloxicam were prepared by spiking the working standard solutions $(1-1000 \mathrm{ng} / \mathrm{mL})$ into a pool of drug-free rat plasma and the procedure described above was followed. Calibration standards consisted of $90 \mu \mathrm{L}$ of pooled drug-free plasma, $10 \mu \mathrm{L}$ of meloxicam standard solution (in $0.1 \%$ aqueous formic acid) and $300 \mu \mathrm{L}$ of acetonitrile containing piroxicam (internal standard at $12.5 \mathrm{ng} / \mathrm{mL}$ concentration). Solutions containing $6.25 \mathrm{ng} / \mathrm{mL}$ and $25 \mathrm{ng} / \mathrm{mL}$ of meloxicam were used as QC samples. $20 \mu \mathrm{L}$ of supernatant was taken out from both of the calibration standards and the QC samples, diluted with $380 \mu \mathrm{L}$ of $0.1 \%$ aqueous formic acid, and $5 \mu \mathrm{L}$ was analysed by LC-MS/MS. 


\subsubsection{In vitro-in vivo correlation calculation}

In vitro-in vivo correlation (IVIVC) is a biopharmaceutical tool for the investigation of the mutual relationship of the dissolution characteristics of the in vitro and in vivo absorption studies (Cardot et al., 2012). In our case, the Pearson's correlation coefficient of the AUC values of the in vitro and in vivo results was calculated by Microsoft Excel (Microsoft Corporation, Redmond, Washington, U.S.) and Statistica for Windows (StatSoft GmbH, Hamburg, Germany). The three prepared samples were correlated with each other in groups of in vitro and in vivo. To determine statistical significance, the unpaired t-test was used

\subsubsection{Statistical analyses}

Data were expressed as means $\pm \mathrm{SD}$, and groups were compared by using Student's t-test. For the evaluation of cell culture results GraphPad Prism 5.0 software (GraphPad Software Inc., San Diego, USA) was used. All culture data presented are means \pm SD, values were compared using analysis of variance followed by Bonferroni posttest. Differences were considered statistically significant when $\mathrm{p}<0.05$.

\section{RESULTS}

\subsection{Optimization of the combined wet milling process}

\subsubsection{Effects of milling parameters on PSD}

The effect of pearl amount and milling time on particle size of $d(0.5)$ was investigated. This study was performed using 2.5\% PVA solution (Bartos et al., 2016). The ratio of the concentrated pre-dispersion (2.0 g of Mel+18.0 g of aqueous PVA solution) and pearl amount was alternated: 1:0.5, 1:1, 1:2, and 1:4 (w/w). It can be established that the pearl amount and the milling time have a great effect of on the $\mathrm{d}(0.5)$ value (Table 6). The milling efficiency was not satisfactory in case of ratio 1:0.5, but it increased linearly on increasing the amount of the milling media and the milling time except in case of ratio 1:4. It was found that the increase of the amount of the milling media (up to $1: 2 \mathrm{w} / \mathrm{w}$ or more) causes gray coloring because of the high friction between the pearls and the wall of the steel jar. Therefore, the pearl milling amount was optimized at the ratio of 1:1 (20 g of concentrated pre-dispersion and $20 \mathrm{~g}$ of pearls), and the milling time was investigated as an independent variable in the factorial experimental design. Another advantage of the small amount of the grinding media may be the reduction of the product loss. 
Table 6. Particle size of Mel $(\mathrm{d}(0.5))$ in milled dispersion as a function of different pearl amount and milling time $(\mathrm{d}(0.5)$ of raw Mel was $34.260 \pm 4.860 \mu \mathrm{m})$

\begin{tabular}{|c|c|c|c|c|}
\hline & \multicolumn{4}{|c|}{ Ratio of pre-dispersion and pearl amount (w/w) } \\
\hline & $1: 0.5$ & $1: 1$ & $1: 2$ & $1: 4$ \\
\hline & \multicolumn{4}{|c|}{ Particle size $(\mu \mathrm{m})$} \\
\hline $10 \mathrm{~min}$ & $4.015 \pm 0.06$ & $2.426 \pm 0.029$ & $2.383 \pm 0.016$ & $0.149 \pm 0.03$ \\
\hline $30 \mathrm{~min}$ & $0.293 \pm 0.008$ & $0.145 \pm 0.007$ & $0.190 \pm 0.003$ & $0.137 \pm 0.006$ \\
\hline $50 \mathrm{~min}$ & $0.202 \pm 0.003$ & $0.140 \pm 0.004$ & $0.140 \pm 0.002$ & $0.130 \pm 0.004$ \\
\hline
\end{tabular}

During the factorial experimental design, the influence of the milling time and the rotation speed on the particle size reduction was investigated. The ratio of the pre-dispersion and the pearls was also 1:1 (w/w). Figure 4. shown that the efficiency of the particle size reduction was improved by increasing the milling time and the rotation speed.

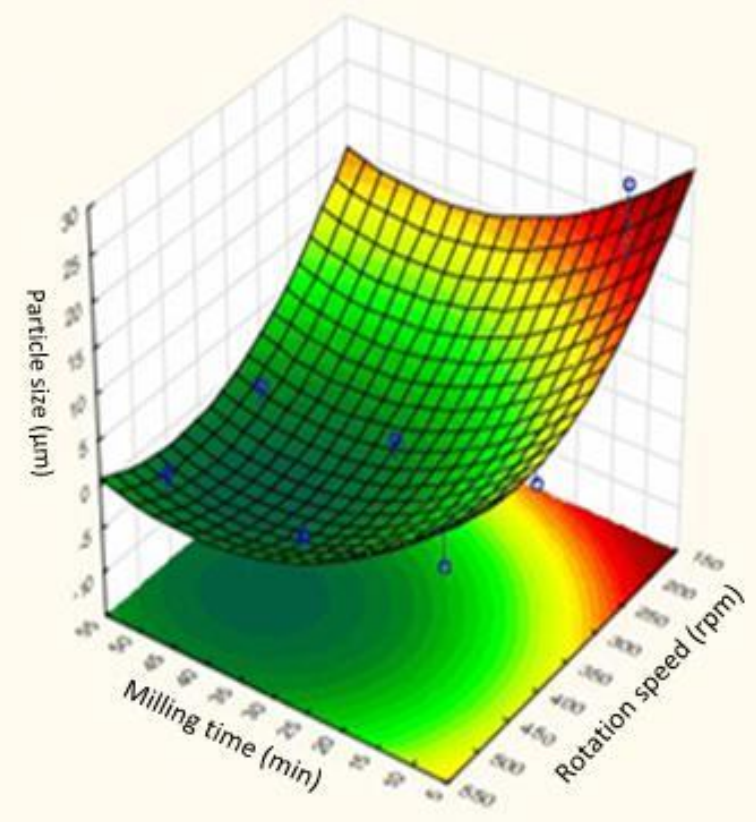

Figure 4. 3D illustration of the particle size changes during the second factorial experimental design

Based on the results, $437 \mathrm{rpm}$ and 43 min are the optimal parameters of the milling process. With 10\% Mel amount and 5\% PVA concentration the pre-dispersion - pearl ratio is 1:1. The PSD values of the sample produced on mathematically pre-determined parameters are as follows: $\mathrm{d}(0.1)-0.067 \pm 0.001 \mu \mathrm{m} ; \mathrm{d}(0.5)-0.130 \pm 0.005 \mu \mathrm{m} ; \mathrm{d}(0.9)-0-371 \pm 0.010 \mu \mathrm{m}$. 


\subsubsection{Effects of PVA on PSD}

Concentrated pre-dispersions with different PVA amounts $(2.5 \%-7.5 \%)$ were milled with optimized parameters ( $2 \mathrm{~g}$ of Mel+18 g of PVA aqueous solution, $20 \mathrm{~g}$ pearls, $437 \mathrm{rpm}, 43$ min). The results show (Figure 5) that the lower concentration of PVA (from 2.5\% to 3.5\%) was not suitable to reach the nanosize range $(<500 \mathrm{~nm})$. A higher concentration of PVA $(6.0 \%-6.5 \%)$ also resulted in unsatisfactory milling effectiveness. Over $6.5 \%$ a robust protecting layer was probably formed on the solid particles, and nanonization was not possible. The curve of the Span values follows the different particle size distribution of the samples. The best particle size distribution was measured in the range of $4.0 \%$ and $5.5 \%$ of PVA. Based on the results, the concentrated milled dispersion containing 5.0\% of PVA was selected as the optimized PVA amount. After the optimization of the wet milling process and the PVA amount, in order to understand the influence of the amount of PVA on the physicochemical properties of the samples, the milled dispersions with 3 different concentrations of PVA were investigated. These were the following: $2.5 \%, 5.0 \%$ (as optimized) and $7.5 \%$ of PVA.

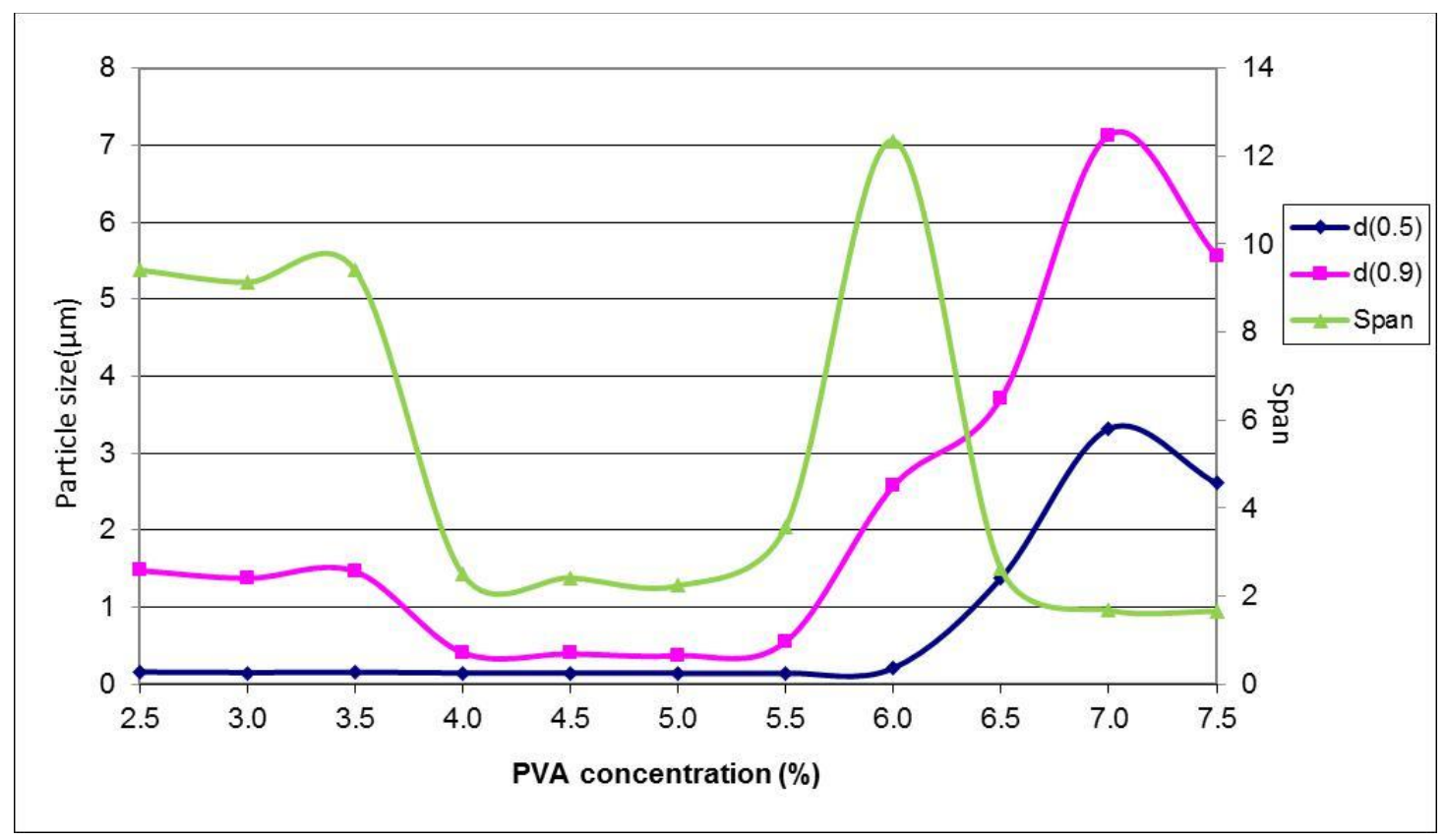

Figure 5. Particle size reduction effectiveness according to PVA concentrations and the Span values for the demonstration of particle size distribution $(\mathrm{d}(0.1)$ values are not shown 


\subsubsection{Rheology}

In order to exclude the viscosity changes because of the high mechanical forces during the milling, the viscosity of 3 raw PVA solutions $(2.5 \%, 5 \%$, and $7.5 \%)$ was investigated before the milling process, after the process (437 rpm, $43 \mathrm{~min}$ ), and after the milling with the addition of Mel. The polymer solutions showed Newtonian flow behavior as their shear viscosity was independent of the applied shear rate. The viscosity of the polymer solutions increased with increasing the polymer concentration (Table 7). There were no remarkable differences between the viscosity of the polymer solution before and after the milling procedure, which may indicate there are no changes in the polymer structure. $10 \% \mathrm{Mel}$ amount in the dispersions increased the viscosity of the systems and did not change the flow behavior - they remained Newtonian. Adding Mel to the polymer solutions, the smallest shift in the viscosity could be detected in case of the highest polymer concentration. This can be explained by the more considerable viscosity changing effect of the polymer concentration than that of the Mel particles. It can be concluded that the mechanical influence did not change the viscosity of the polymer solutions, and therefore, the structure of the PVA chains did not change. 
Table 7. Viscosity values ( $\eta$ ) of the raw PVA solutions before milling (PVA \%), after milling (PVA \% milled) and the PVA solutions after milling in addition with Mel (Mel PVA \% milled)

\begin{tabular}{|l|c|c|}
\hline & $\eta$ & $\mathrm{SD} \pm$ \\
\hline PVA 2.5\% & $\mathrm{mPa}^{*} \mathrm{~s}$ & $\mathrm{mPa}^{*} \mathrm{~s}$ \\
\hline PVA 5.0\% & 3.14 & 0.02 \\
\hline PVA 7.5\% & 7.38 & 0.02 \\
\hline PVA 2.5\% milled & 20.55 & 0.07 \\
\hline PVA 5.0\% milled & 3.12 & 0.27 \\
\hline PVA 7.5\% milled & 20.05 & 0.02 \\
\hline Mel PVA 2.5\% milled & 4.42 & 0.07 \\
\hline Mel PVA 5.0\% milled & 8.46 & 0.07 \\
\hline Mel PVA 7.5\% milled & 21.65 & 0.07 \\
\hline
\end{tabular}

\subsubsection{DSC}

DSC was used to investigate the melting of raw Mel and raw PVA and the dried samples (Figure 6). The DSC curve of the raw Mel revealed a sharp endothermic peak at $268.66^{\circ} \mathrm{C}$, reflecting its melting point and an instantly following exothermic peak at $278.09^{\circ} \mathrm{C}$ can be observed. The DSC curve of raw PVA as a semi-crystalline polymer has 2 endothermic peaks at $169.51{ }^{\circ} \mathrm{C}$ and at $222.74^{\circ} \mathrm{C}$. The first peak of PVA signifies a particular decrystallization of PVA and the second peak is the melting temperature (Tretinnikov et al., 2012). The DSC curves of the dried products exhibited lower melting points of Mel than that of raw Mel. It is connected to the pre-melting of PVA, which induces the earlier melting of Mel in proportion to the amount of PVA and decreases the crystallinity degree of Mel 


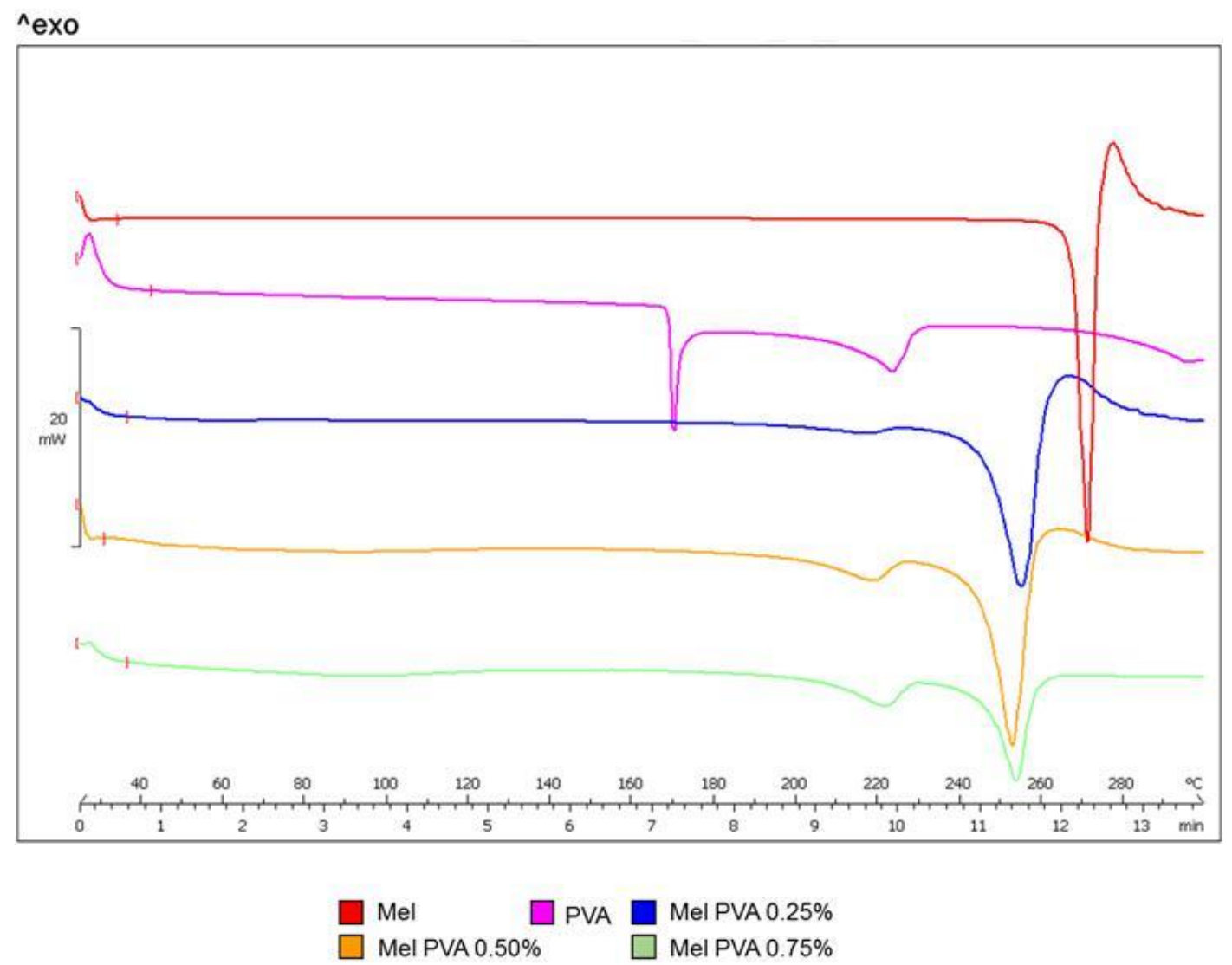

Figure 6. DSC curves of raw Mel and PVA and dried dispersions with different PVA concentrations

\subsubsection{XRPD}

The XRPD investigations justified the change of crystallinity degree of Mel in the dispersions with different PVA amounts. Figure 7 shows the fingerprints of raw materials (Mel and PVA) and the dried dispersions. The samples show the characteristic peaks of Mel at $2 \theta$ values: $13.06^{\circ}, 14.94^{\circ}$, and $18.61^{\circ}$. It was found that the PVA content of the samples fundamentally influenced the decrease of the crystallinity degree of Mel. As it was established earlier in this study, low (2.5\%) and high (7.5\%) concentrations of PVA in the milled dispersion did not result in suitable milling efficiency. In this case, the crystallinity degree of Mel was $75.82 \%$ at low PVA content $(2.5 \%)$, and it decreased to $51.44 \%$ at high concentration of PVA $(7.5 \%)$. These results are connected to the milling effectiveness. In this study, the 5.0\% PVAcontaining milled dispersions showed smaller crystallinity (13.43\%) and the highest milling efficiency. 


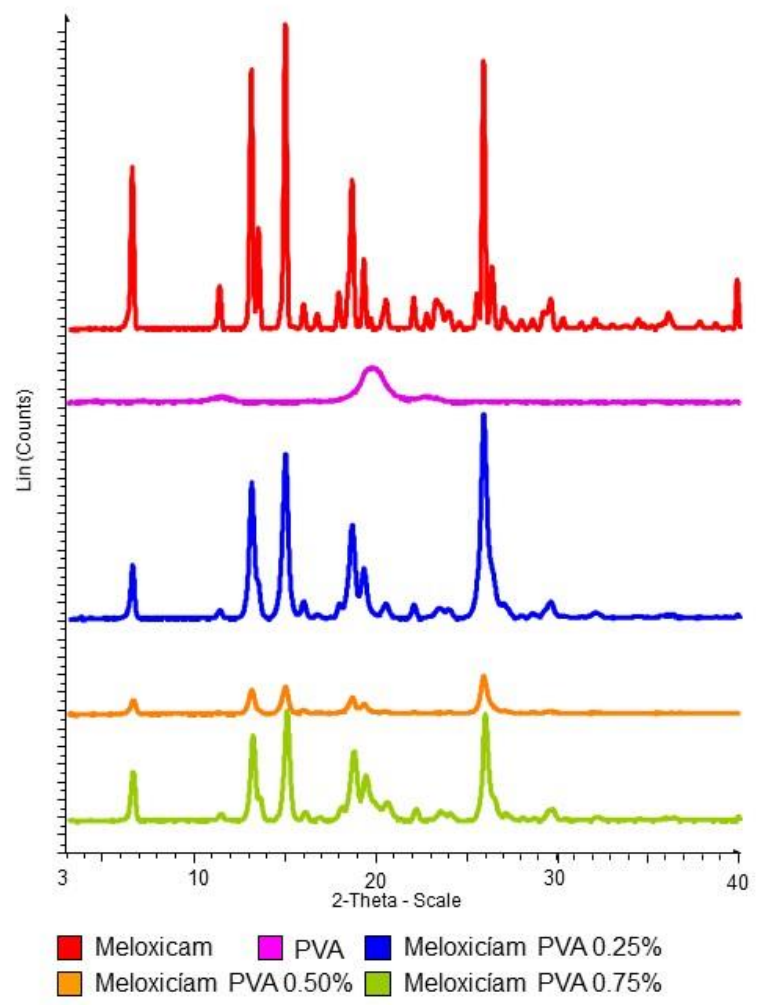

Figure 7. XRPD diffractograms of the Mel, PVA and the dried dispersion

\subsubsection{Zeta potential changes}

To determine the electrokinetic changes of the diluted dispersions, the zeta potential of the samples was measured. The results show that the increase of the PVA amount decreases the zeta potential in comparison to the sample without PVA (Table 8). The main reason for the zeta potential reduction can be linked to the nonionic polymer adsorption on the surface of the solid particles, which causes a decrease of the diffuse layer charge. A greater zeta potentiallowering effect can be observed between $0 \%$ and $0.25 \%$ of PVA than between $0.25 \%$ and 0.50 . At $0.50 \%$ of PVA, the surface of particles is saturated by the PVA chain; therefore, the change of zeta potential is smaller. In case of a higher concentration of PVA $(.0 .50 \%)$, the steric hindrance stabilizes the system but hampers the disintegration/abrasion of the particles. 
Table 8. Zeta potential values of the diluted dispersions as a function of the PVA concentration and particle size distribution of Mel $( \pm \mathrm{SD})$

\begin{tabular}{|c|c|c|c|c|}
\hline \multirow{3}{*}{ Samples } & \multicolumn{3}{|c|}{ Particle size distribution } & \multirow{3}{*}{$\begin{array}{l}\text { Zeta } \\
\text { potential } \\
(\mathrm{mV})\end{array}$} \\
\hline & $\mathrm{d}(0.1)$ & $\mathrm{d}(0.5)$ & $\mathrm{d}(0.9)$ & \\
\hline & \multicolumn{3}{|c|}{ Particle size $(\mu \mathrm{m})$} & \\
\hline Mel PVA 0\% & $2.508 \pm 1.100$ & $5.762 \pm 2.700$ & $\begin{array}{l}135.640 \pm 12.90 \\
0\end{array}$ & -30.7 \\
\hline $\begin{array}{ll}\text { Mel } & \text { PVA } \\
0.25 \% & \end{array}$ & $0.070 \pm 0.001$ & $0.150 \pm 0.009$ & $1.478 \pm 0.0400$ & -20.9 \\
\hline $\begin{array}{ll}\text { Mel } & \text { PVA } \\
0.50 \% & \end{array}$ & $0.067 \pm 0.001$ & $0.130 \pm 0.005$ & $0.371 \pm 0.010$ & -16.1 \\
\hline $\begin{array}{ll}\text { Mel } & \text { PVA } \\
0.75 \% & \end{array}$ & $1.235 \pm 0.006$ & $2.611 \pm 0.018$ & $5.560 \pm 0.070$ & -15.7 \\
\hline
\end{tabular}

\subsubsection{Raman spectroscopy}

Raman spectra and chemical maps of raw materials and products are presented in Figures 8 and 9. The individual spectrum of Mel (a) shows that the absorption peaks are concentrated in the region from 1,600 to $1,000 \mathrm{~cm}^{-1}$ (fingerprint region), whereas the individual spectra of PVA (b and c) show only one characteristic and extended peak from 3,000 to 2,800 cm-1. This is the $\mathrm{CH}$ stretching vibration region that results a medium-to-strong intensity in Raman spectra. The spectra of diluted dispersions (d, e, and f) show the same characteristic region with the spectra of Mel - there are no detectable differences among them. Two peaks (one more characteristic in $2,437 \mathrm{~cm}^{-1}$ and another smaller one in $482 \mathrm{~cm}^{-1}$ ) appear in the spectra of PVA-containing dispersions and in spectra of aqueous Mel dispersion as well. The chemical mapping of dried dispersions profiled to this peak in $2,437 \mathrm{~cm}^{-1}$ shows that this peak cannot detected in this map. This peak can show a dissociated -OH group of enolic form which is presented when Mel is in an aqueous medium, but it is disappeared in dried form, so the change is reversible. The chemical map of dispersion profiled to Mel spectrogram shows homogenous distribution of Mel. It can be summarized that there is no chemical degradation or interaction in dispersion which could be detectable with Raman technique. 


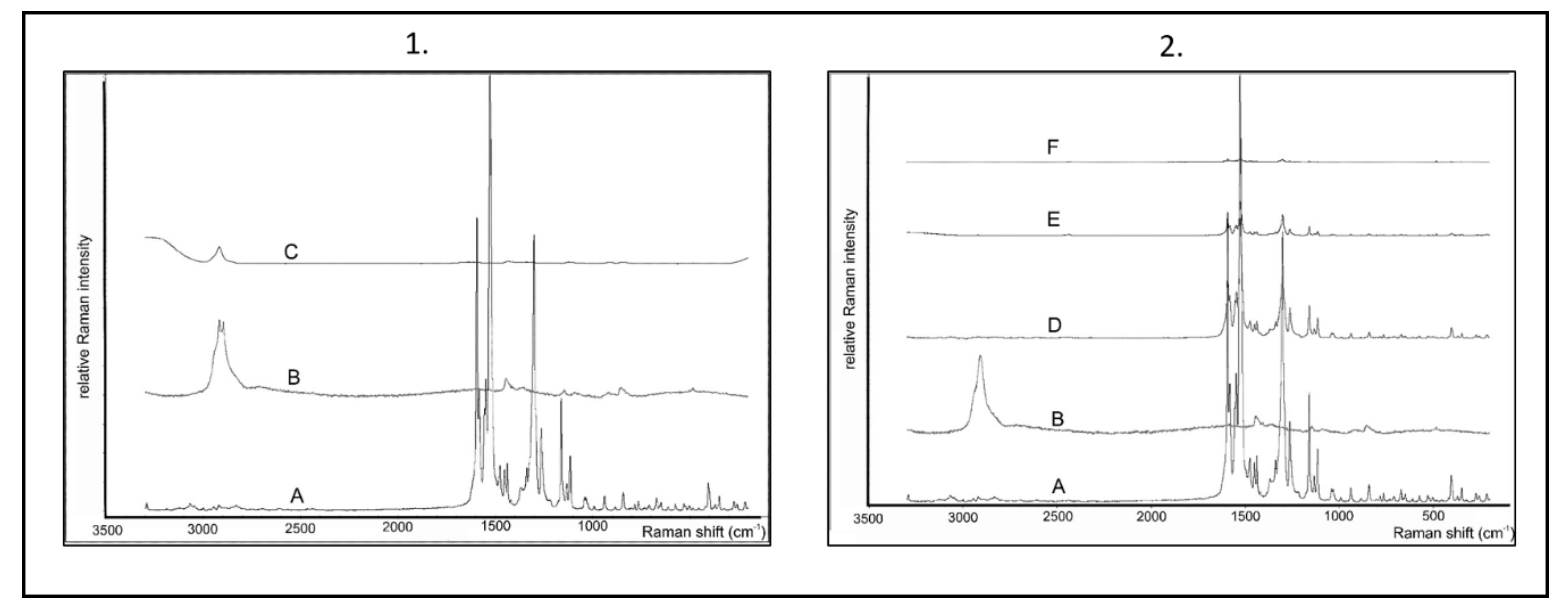

Figure 8. Investigation with Raman spectroscopy: 1. A: spectrum of raw Mel, B: spectrum of raw PVA, C: spectrum of raw PVA (0.50\%) containing solution. 2. Comparison study of raw materials (Mel, PVA) and the dispersions, D: spectrum of dispersion containing $1 \% \mathrm{Mel}$ and $0.25 \%$ PVA, E: spectrum of dispersion containing $1 \% \mathrm{Mel}$ and $0.50 \%$ PVA, F: spectrum of dispersion containing $1 \% \mathrm{Mel}$ and $0.75 \% \mathrm{PVA}$

1.

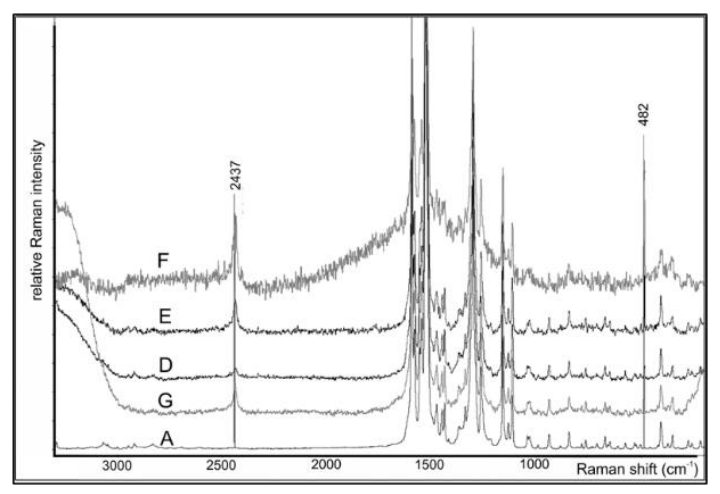

2.

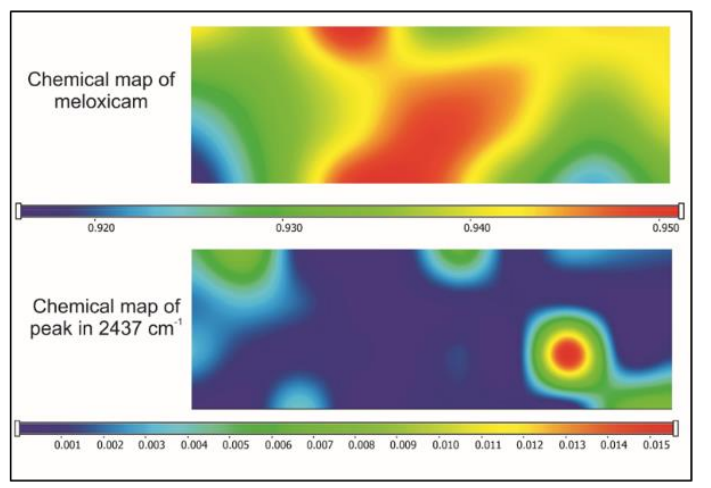

Figure 9. Investigation with Raman spectroscopy: 1. Comparing raw Mel and aqueous dispersion of Mel and PVA containing dispersions, A: spectrum of raw Mel, D: spectrum of dispersion containing $1 \% \mathrm{Mel}$ and $0.25 \%$ PVA, E: spectrum of dispersion containing $1 \% \mathrm{Mel}$ and $0.50 \%$ PVA, F: spectrum of dispersion containing $1 \% \mathrm{Mel}$ and $0.75 \%$ PVA, G: spectrum of aqueous $1 \% \mathrm{Mel}$ containing dispersion without PVA. 2. Chemical mapping of Mel 
containing dispersion (1\% Mel and 0.50\% PVA) and chemical mapping of its dried form profiled to peak in $2437 \mathrm{~cm}^{-1}$

\subsubsection{SEM investigation}

In order to investigate the effect of milling and PVA amount on the morphology of milled Mel, the water was evaporated from dispersion and the dried samples were characterized. Figure 10 shows the raw Mel in physical mixture (Mel PVA 0.50\% PM) which has an irregular shape with $34.260 \pm 4.860 \mu \mathrm{m}$ as average particle size. In this PM, the PVA particles with size, $6 \mu \mathrm{m}$ are located on the surface of Mel crystals. After drying of the aqueous dispersions, the polymer formed non-coherent and coherent think film with Mel particles. In case of $0.25 \%$ containing PVA (Mel PVA $0.25 \%$ ), the particle size of Mel has decreased, but aggregation of the fragmented particles can be observed. Mel particles are in homogeneous disperse distribution in sample (Mel PVA 0.50\%) resulted in less than $500 \mathrm{~nm}$ (average particle size: $0.130 \pm 0.005 \mu \mathrm{m}$ ). High concentration of PVA (Mel PVA $0.75 \%$ ) helped the recrystallization of $\mathrm{Mel}$ (nanocrystals with smooth surface) thanks to increased solubility of Mel in aqueous PVA solution. Otherwise, the sample shows heterogeneous disperse system with nano- and microparticles.

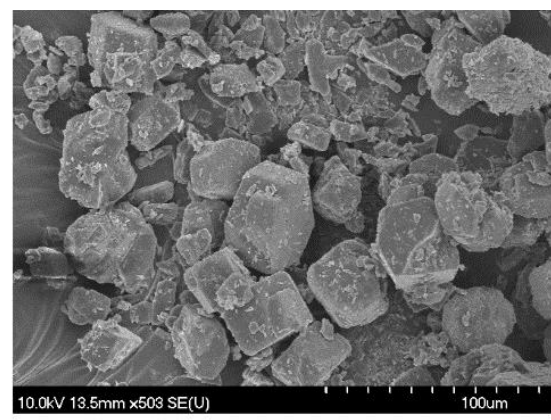

Mel PVA $0.25 \%$ PM

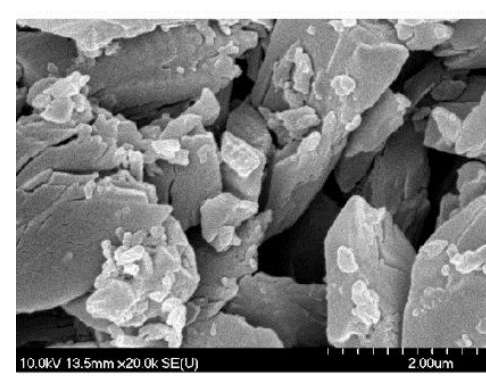

Mel PVA $0.25 \%$

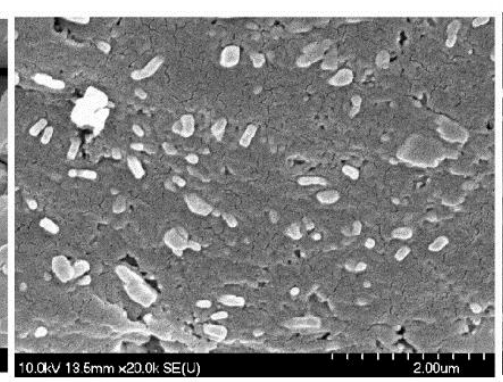

Mel PVA $0.50 \%$

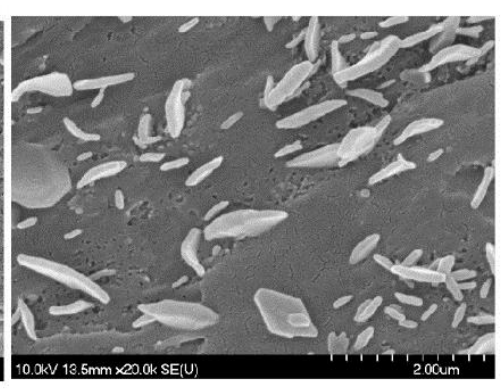

Mel PVA $0.75 \%$

Figure 10. SEM pictures of physical mixture (Mel PVA $0.25 \%$ PM) and different PVA containing dried dispersions 


\subsubsection{In vitro studies of the nanosuspensions}

In case of each sample, the liberation from the hard gelatin capsules occurred within 2 sec. Mel has a weak acidic character ( $\mathrm{pKa} 4.8$ ), and therefore, its solubility in gastric juice $(\mathrm{pH}=1.2)$ is very poor $\left(1.6 \pm 0.2 \mathrm{mg} / \mathrm{L}\right.$, at $\left.37^{\circ} \mathrm{C}\right)$. In this medium, the greatest dissolved amount of Mel with $0.25 \%$ and $0.50 \%$ PVA content was maximum, 37\%, within 20 min (Figure 11A). This result is due to the wetting effect of PVA $(0.25 \%$ and $0.50 \%)$, which could increase the solubility of Mel, and the reduction of the particle size of Mel in the dispersions. In contrast, a higher amount of PVA $(0.75 \%)$ hinders the dissolution because a thicker polymer layer is formed on the Mel particles.

In intestinal fluid ( $\mathrm{pH}=6.8)$, the dissolved amount was higher in all cases because of the better solubility of $\mathrm{Mel}\left(0.272 \pm 0.001 \mathrm{mg} / \mathrm{mL}\right.$, at $\left.37^{\circ} \mathrm{C}\right)$. Figure $11 \mathrm{~B}$ shows that the concentration of PVA influences the amount of dissolved. Mel as in the case of gastric juice, but its extent is bigger. In this study, the dispersion containing $0.50 \%$ of PVA had the most satisfying dissolution behavior from among the 4 samples. It is followed by the dispersions containing $0.25 \%$ and $0.75 \%$ of PVA, and finally the dispersion without PVA. The results justify the need of the polymer (PVA) and the correct choice of its quantity.
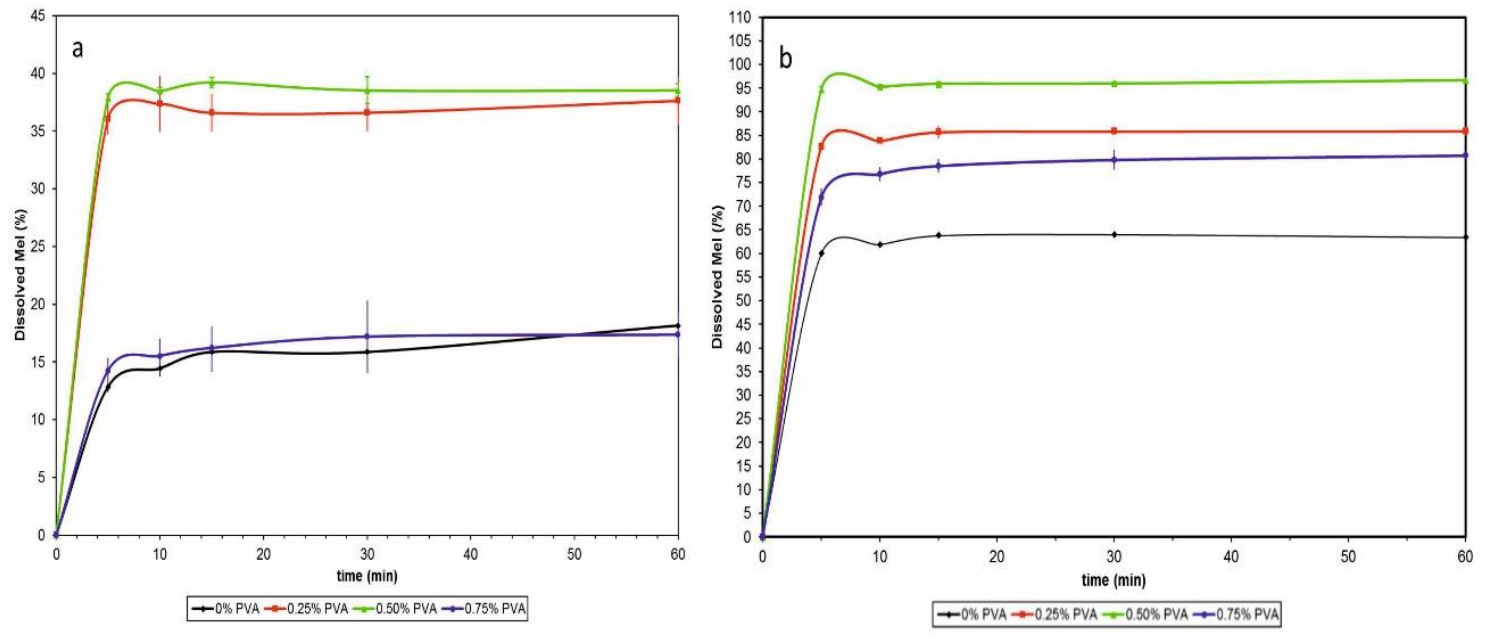

Figure 11. In vitro dissolution curves of $\mathrm{Mel}$ in artificial gastric juice (a) and intestinal juice (b)

\subsubsection{In vitro cell culture studies}

Impedance measurement, as a sensitive method to detect cellular effects, did not show significant cell damage after treatments with Mel, PVA, and Mel formulation groups, as 
reflected by unchanged cell index values (Figure 12). As a comparison, cells treated with the detergent Triton X-100 were lysed and a 100\% toxicity was measured. The curves show the kinetics of the cellular effects of treatment solutions (Figure 12A), whereas the columns show the effect of Mel, PVA and Mel formulations at the 8-h time point (Figure 12B).

Caco-2 monolayers showed high TEER values $(2,660 \pm 181 \Omega \times \mathrm{cm} 2, \mathrm{n}=20)$ before permeability experiments, indicating tight barrier properties. The average apparent permeability coefficients of marker molecules were also low (fluorescein: $0.81 \pm 0.13 \times 10-6$ $\mathrm{cm} / \mathrm{s}$; albumin: $0.08 \pm 0.03 \times 10-6 \mathrm{~cm} / \mathrm{s}$ ), in agreement with the TEER values and the formation of tight cell layers.

The permeability of Mel suspension and Mel formulations was significantly higher than that of marker molecules. The penetration of Mel from the 3 investigated products across cell layers was significantly increased as compared to Mel suspension. From all the tested samples, the Papp value of Mel was the highest in the formulation containing $0.05 \%$ PVA (Figure 13).
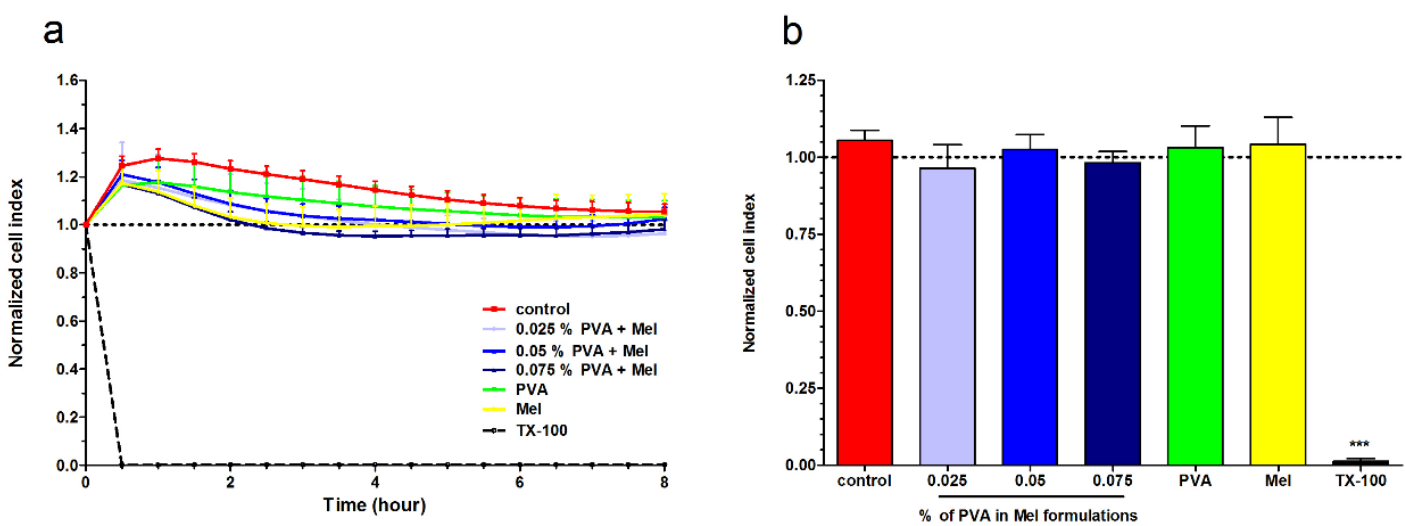

Figure 12. Cell viability kinetics (a) and results 8 hours after treatment (b) of Caco-2 intestinal epithelial cells with Mel, PVA and formulations measured by impedance. Values are presented as means $\pm \mathrm{SD}, \mathrm{n}=6-12$. Statistical analysis: ANOVA followed by Dunett's test. Statistically significant differences are: ${ }^{*}, p<0.05$, compared to control group. 


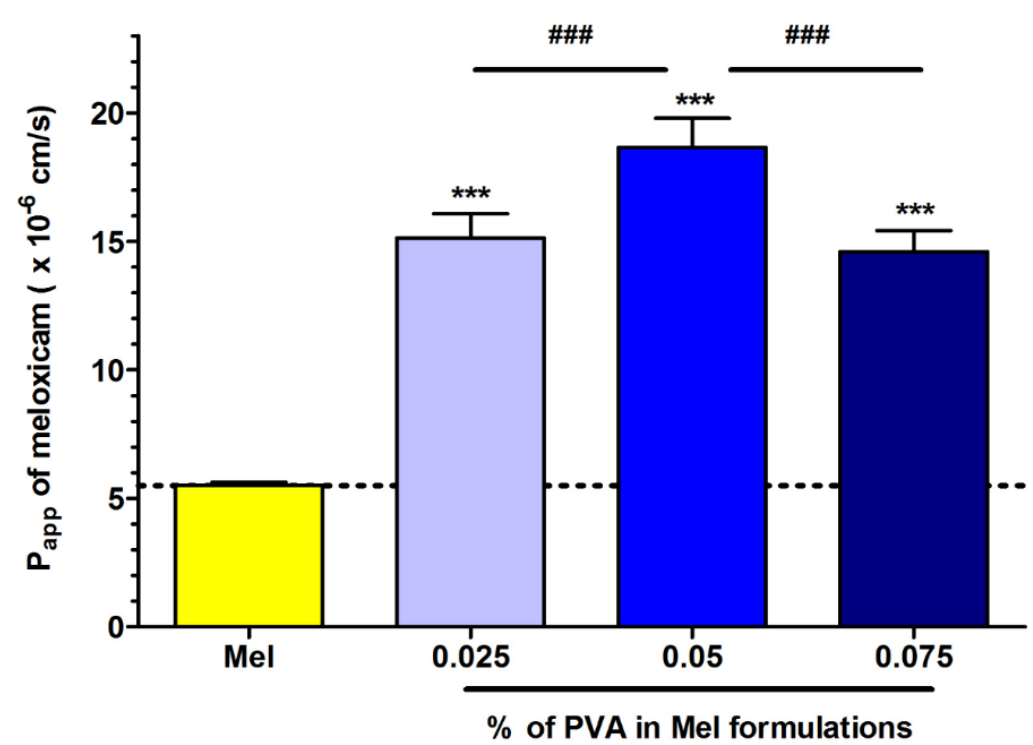

Figure 13. Evaluation of permeability of meloxicam across Caco-2 epithelial cell layers treated with Mel and optimized Mel-PVA formulations for 1 hour. Values are presented as means \pm SD, $n=4$. Statistical analysis: ANOVA followed by Bonferroni posttest. Statistically significant differences are: $* * *, p<0.01$, compared to control group; \#\#\#, $p<0.01$ compared to the indicated columns.

\subsubsection{Holding time determination}

Since the investigated dispersions are intermediate products, the change of particle size distribution and the crystallinity index are very important during storage. In general, the time period before the dispersion used for the preparation of different dosage forms is 1 or $2 \mathrm{~h}$, or possibly longer. The measurements have proven that $0.50 \%$ of PVA-containing dispersion had no significant changes in the particle size, particle size distribution, and crystallinity up to 2 weeks (Table 9). 
Table 9. Particle size distribution changes $(\mu \mathrm{m})$ during the stability testing

\begin{tabular}{|l|l|l|l|l|l|l|}
\hline & \multicolumn{5}{|l|}{ Day 0 } & \multicolumn{2}{l|}{ weeks } \\
\hline & $\mathrm{d}(0.1)$ & $\mathrm{d}(0.5)$ & $\mathrm{d}(0.9)$ & $\mathrm{d}(0.1)$ & $\mathrm{d}(0.5)$ & $\mathrm{d}(0.9)$ \\
\hline Mel PVA 0.25\% & 0.070 & 0.150 & 1.478 & 0.080 & 0.152 & 2.073 \\
\hline Mel PVA 0.50\% & 0.067 & 0.136 & 0.371 & 0.068 & 0.140 & 0.427 \\
\hline Mel PVA 0.75\% & 1.207 & 2.232 & 5.224 & 0.244 & 2.611 & 5.560 \\
\hline
\end{tabular}

\subsection{Results of the robustness testing experiments}

\subsubsection{Effect of the API content on the PSD}

Using the optimized process parameters, based on the change in the amount of Mel, the particle size distribution is one of the critical parameters (Bartos at al.. 2019). In this respect, the comparison is based on the particle size of the optimized sample "Mel_2.0g", which had $10 \%$ of MEL and $5 \%$ of PVA (Table 10).

After milling, the different Mel amounts containing nanosuspensions showed same $d(0.5)$ value, which means that the maximum particle diameter of $50 \%$ of the sample volume is less than $150 \mathrm{~nm}$. By contrast, the d(0.9) values, in the case of smaller and higher amounts of Mel, were already outside the upper range of the desired value $(500 \mathrm{~nm})$. It can be assumed that the smaller amount of Mel $(<10 \%)$ decreased the number of successful collisions of Mel and the milling media. The larger amount of Mel (>17.5\%), however, increased the density of the sample and reduced milling efficiency. Span values, which contain the $\mathrm{d}(0.1)$ values, confirm the suitability of particle size distribution in the range of 10\% (Mel_2.0g) and 17.5\% of Mel (Mel_3.5g). 
Table 10. Results of the particle size measurements

\begin{tabular}{|l|l|l|l|}
\hline Sample name & $\mathrm{d}(0.5) / \mu \mathrm{m}$ & $\mathrm{d}(0.9) / \mu \mathrm{m}$ & Span \\
\hline Mel_raw & $34.26 \pm 4.86$ & $73.59 \pm 27.11$ & 1.815 \\
\hline Mel_0.5g & $0.138 \pm 0.027$ & $1.273 \pm 0.134$ & 8.662 \\
\hline Mel_1.0g & $0.142 \pm 0.023$ & $1.949 \pm 0.117$ & 10.207 \\
\hline Mel_1.5g & $0.141 \pm 0.018$ & $0.87 \pm 0.076$ & 5.676 \\
\hline Mel_2.0g* & $0.136 \pm 0.003$ & $0.371 \pm 0.043$ & 2.240 \\
\hline Mel_2.5g & $0.139 \pm 0.006$ & $0.413 \pm 0.037$ & 2.655 \\
\hline Mel_3.0g & $0.145 \pm 0.008$ & $0.436 \pm 0.045$ & 2.956 \\
\hline Mel_3.5g & $0.141 \pm 0.013$ & $0.448 \pm 0.050$ & 2.700 \\
\hline Mel_4.0g & $0.140 \pm 0.021$ & $0.974 \pm 0.072$ & 6.448 \\
\hline
\end{tabular}

* optimized sample

5.2.2. Crystallinity changes according to the API content

\subsubsection{DSC measurements}

For the crystallinity characterization of Mel, first raw Mel and PVA were investigated (Figure 14). The DSC curve of raw Mel has a sharp characteristic melting peak at $268.66^{\circ} \mathrm{C}$ (onset266.83, endset-273.27 ${ }^{\circ} \mathrm{C}$ ), and an instantly following exothermic peak can be observed at $279.09{ }^{\circ} \mathrm{C}$. PVA has two endothermic peaks at $169.51{ }^{\circ} \mathrm{C}$ and at $222.74{ }^{\circ} \mathrm{C}$. The first peak belongs to a particular de-crystallization and the second one presents the melting point of PVA. It should be noted that PVA has deacetylation in the temperature range $160-400{ }^{\circ} \mathrm{C}$ and the total degradation corresponds to the degradation of vinyl acetate and vinyl pyrrolidone at $396{ }^{\circ} \mathrm{C}$ and $484^{\circ} \mathrm{C}$, respectively (McNeill et al., 1995).

The DSC curves of the samples exhibit that the increase of the Mel amount results in a sharper endothermic peak, approaching the melting point of raw Mel. By decreasing the Mel amount, the endothermic peaks will be less sharp because of the pre-melting of the higher 
amount of PVA, which induces the earlier melting of Mel in proportion to the PVA amount. The areas of the melting enthalpies already predict the change in the degree of crystallinity of Mel.

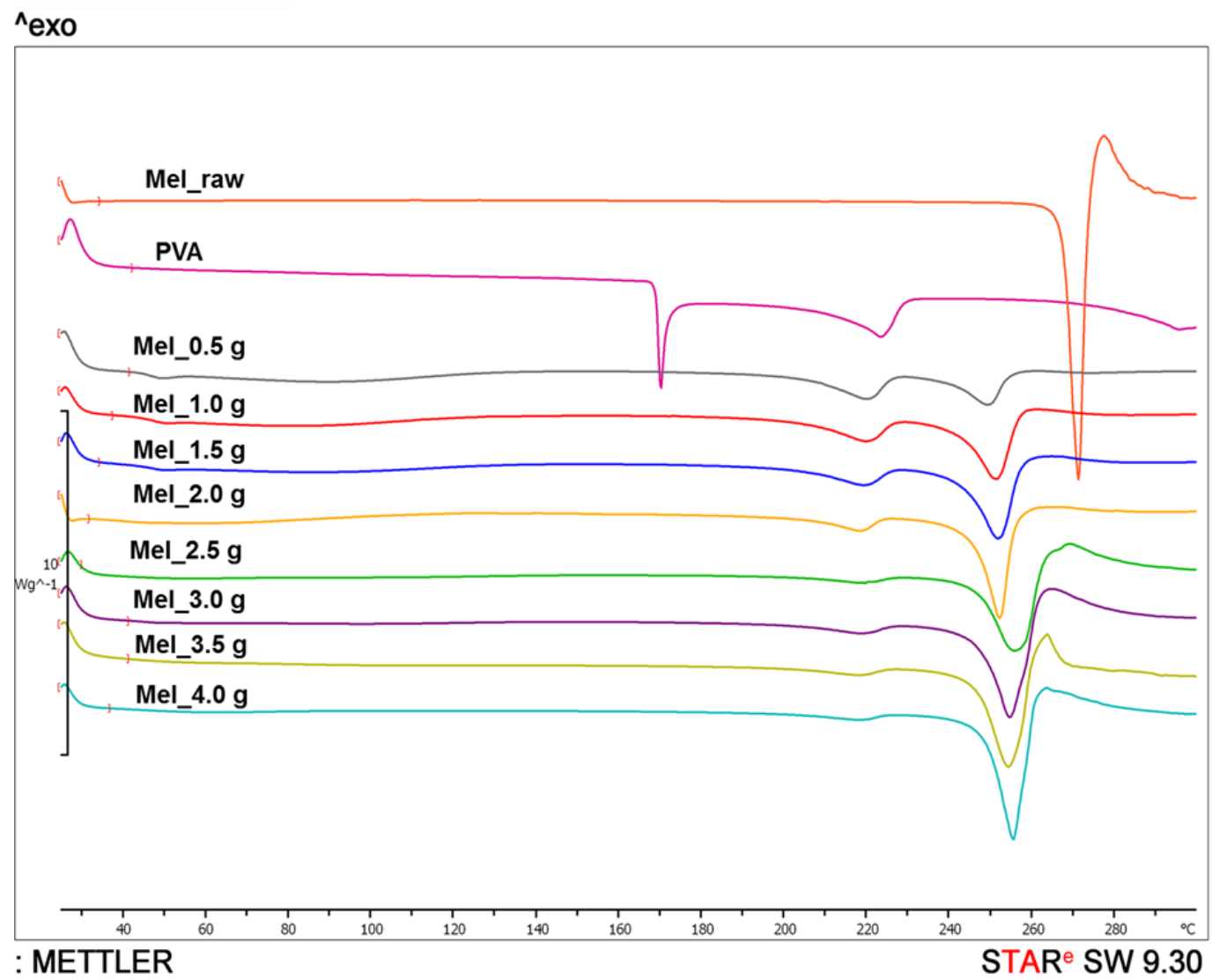

Figure 14. DSC curves of raw Mel, PVA and different Mel containing milled samples

In order to determine the degree of crystallinity of Mel, considering the influence of PVA, the physical mixtures of the milled samples formed the basis.

The Figure 15 presents the degree of crystallinity as a function of the Mel amount in the sample. By increasing the Mel amount, the crystallinity of the milled products was increased. A close correlation $\left(\mathrm{R}^{2}=0.9587\right)$ was found between crystallinity and the Mel amount. It can be seen that increasing the amount of Mel (> 10\%) significantly increased the degree of crystallinity of the milled sample (from $21.21 \%$ to $48.86 \%$ ). However, the small amount of Mel lowered this value (from $21.21 \%$ to 10.28 ). 


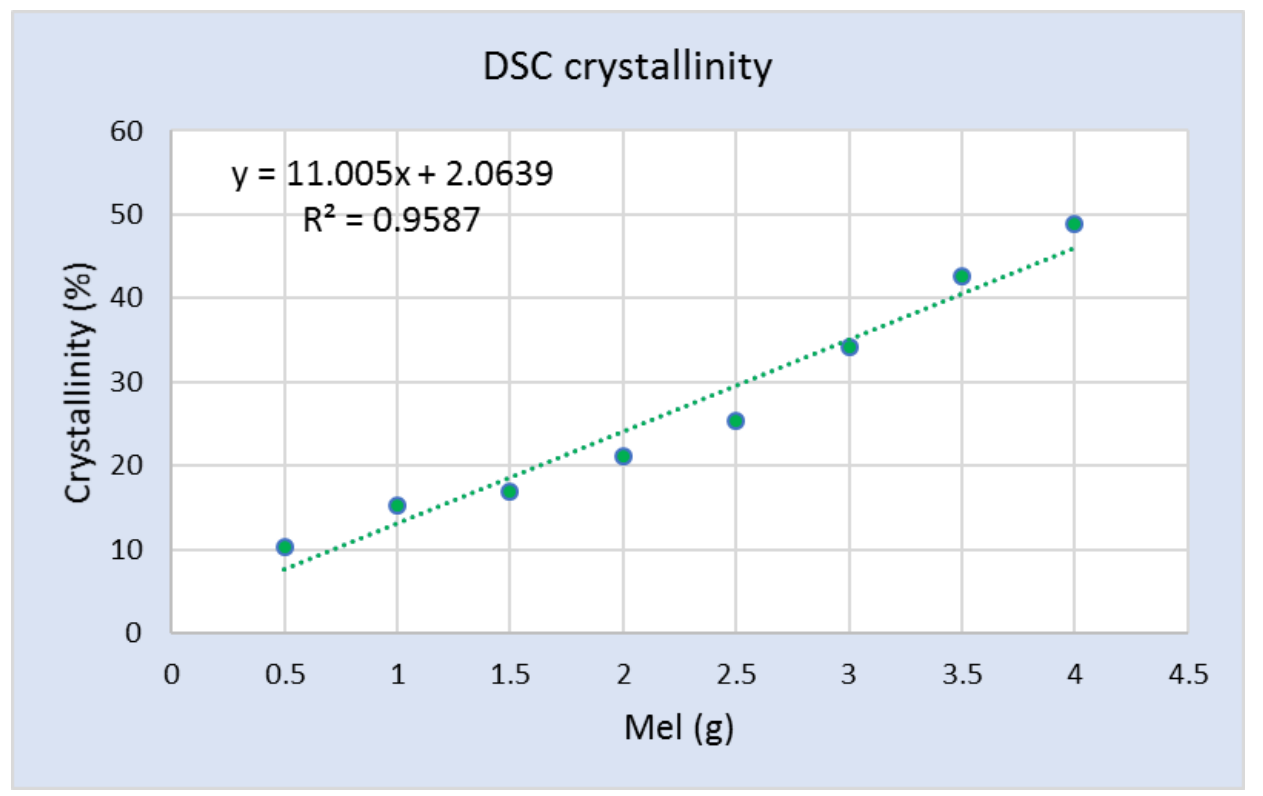

Figure 15. Relation between the crystallinity of Mel and its amount in the milled samples

\subsubsection{XRPD measurements}

To verify the DSC results, the crystallinity of the physical mixtures and the milled samples was investigated by XRPD analysis. Figure 16 presents the fingerprints of raw Mel and PVA and the milled samples. The characteristic peaks of Mel are at $2 \Theta$ value: $13.06^{\circ}, 14.94^{\circ}$ and $18.61^{\circ}$ and PVA has the typical diffraction peak at $2 \Theta$ value: $19.9^{\circ}$ (Garcia-Cerda et al., 2007).

The intensity of the characteristic peaks shows a change in the crystallinity of the milled products. The quantitative analysis of the degree of crystallinity also resulted in a close correlation $\left(\mathrm{R}^{2}=0.9763\right)$ between the degree of crystallinity and the Mel amount (Figure 17). 


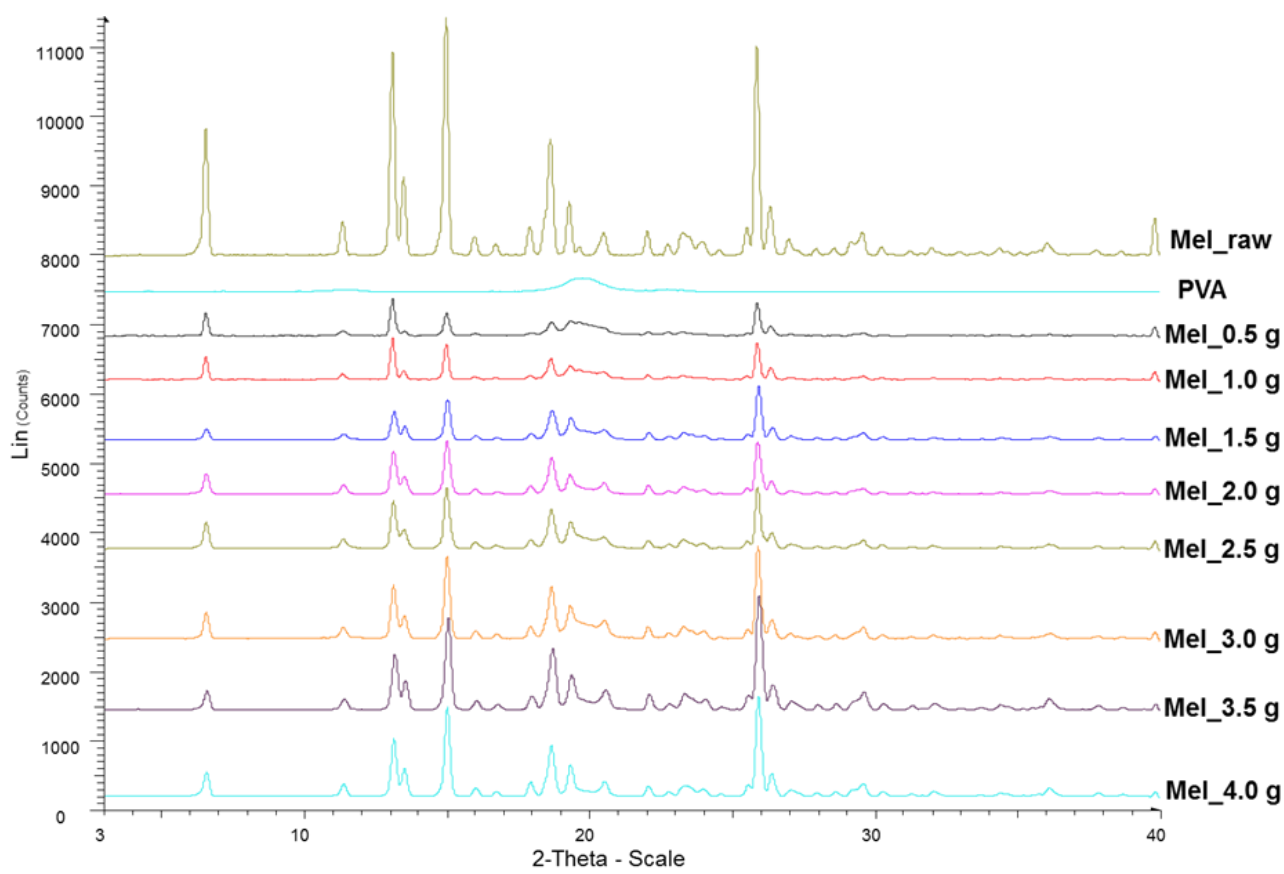

Figure 16. XRPD curves of raw Mel, PVA and different Mel containing milled samples

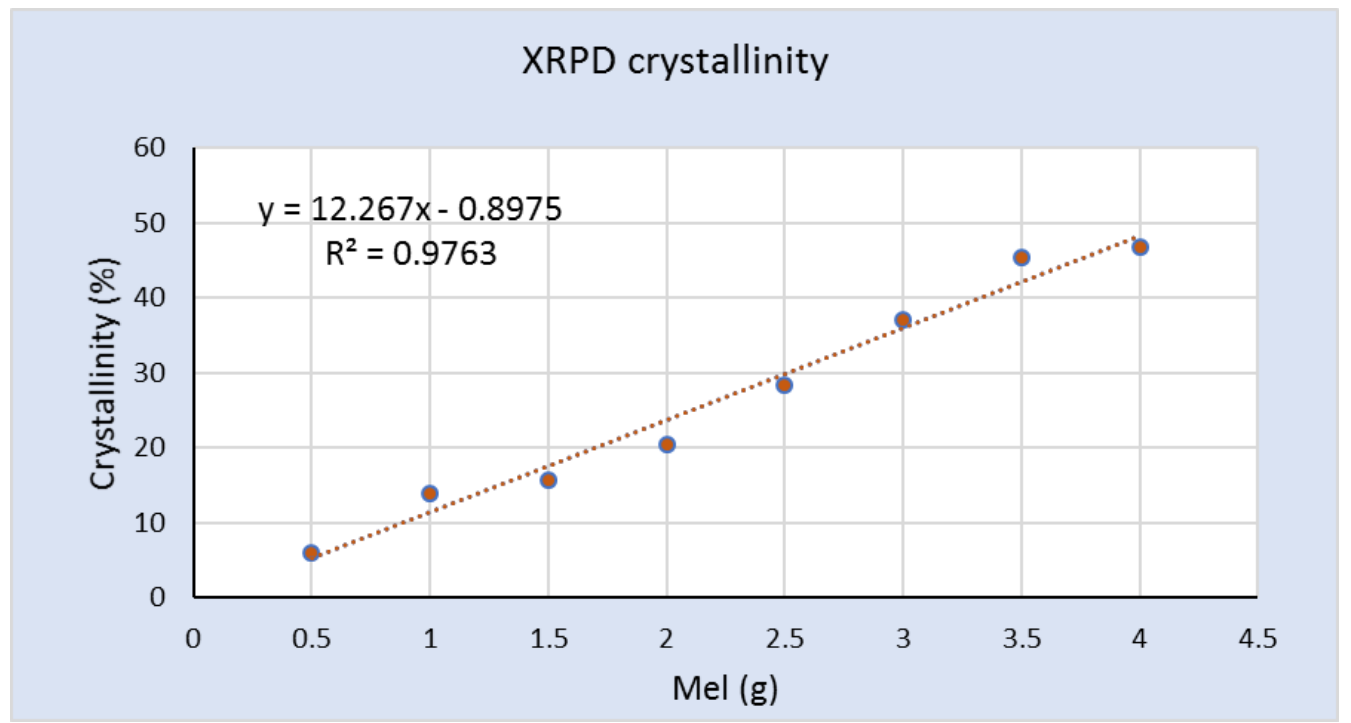

Figure 17. Relation between the crystallinity of Mel and its amount in the milled samples

\subsubsection{Correlation between the two analytical methods}

As the result of the correlation calculations, it was found that the degree of crystallinity of Mel investigated by DSC and XRPD did not show any significant difference at 95\% significance level. It can be related to the low degree of crystallinity $(<50 \%)$. It is known that 
the advantage of DSC over X-ray diffraction is that high amorphous content can be detected (Gombás et al., 2002). It should be noted that recently the characterization of the investigated materials (e.g. crystallinity) using DSC as a semi-quantitative conventional method has become common in scientific research (Katona et al., 2016, Gieszinger et al., 2017, Bartos et al., 2018). It provides a possibility to predict the crystallinity of the examined materials, but to verify the accuracy of thermoanalytical measurements, the X-ray powder diffraction test is required (Dávid et al., 2000).

\subsection{Results of the solid-state product characterizations}

\subsubsection{Particle size measurements}

Different techniques have been used to determine the particle size of Mel for reasons of accuracy and comparability. The particle size distribution of the rawMel and nanoMel samples was investigated via laser diffraction. A combined wet milling process resulted in a 200 -fold particle size reduction in the case of nanoMel $(\mathrm{d}(0.50), 130 \pm 5 \mathrm{~nm})$ compared to the raw drug particle size $(\mathrm{d}(0.50), 34.26 \pm 4.86 \mu \mathrm{m})$. The nanoMel product showed a monodisperse distribution $(\mathrm{d}(0.10), 67 \pm 1 \mathrm{~nm} ; \mathrm{d}(0.50), 130 \pm 5 \mathrm{~nm} ; \mathrm{d}(0.90), 371 \pm 12 \mathrm{~nm})$.

For fluidMel sample, Mel particles adhered to the carrier surface (MCC) was analyzed by Image J technique and the particle size of Mel in the nanoMel and lyoMel samples was compared with dynamic light scattering technique (Malvern nanoZS), too. The results demonstrate that the $\mathrm{d}(0.50)$ value of the Mel nanoparticles measured on the surface of solid phase product (fluidMel) does not show a significant difference regarding the $\mathrm{d}(0.50)$ value of the nanoMel (Figure 18A). In the case of lyoMel, compared to the Z-average of Mel in nanoMel, a significant difference can already be detected which was caused by the recrystallization of the Mel (Figure 18B). Both samples have a same polydispersity index (nanoMel: 0.273 and lyoMel: 0.287 ) which also confirms the monodispersity of nanoMel and shows the excellent redispersibility of lyoMel. The 6-month storage did not cause any further changes in the mean particle size of the products (Figure 18A, 18B). 


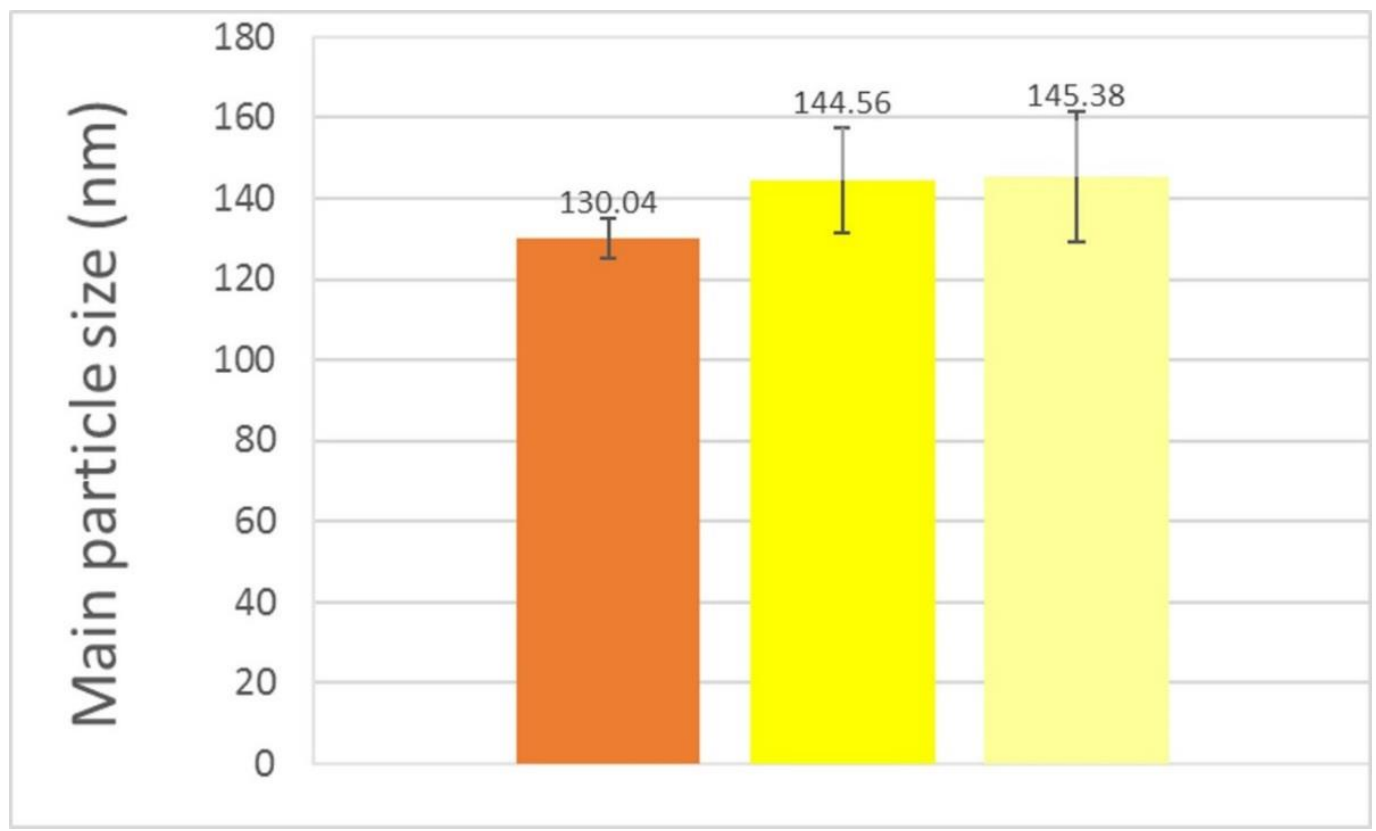

(A)

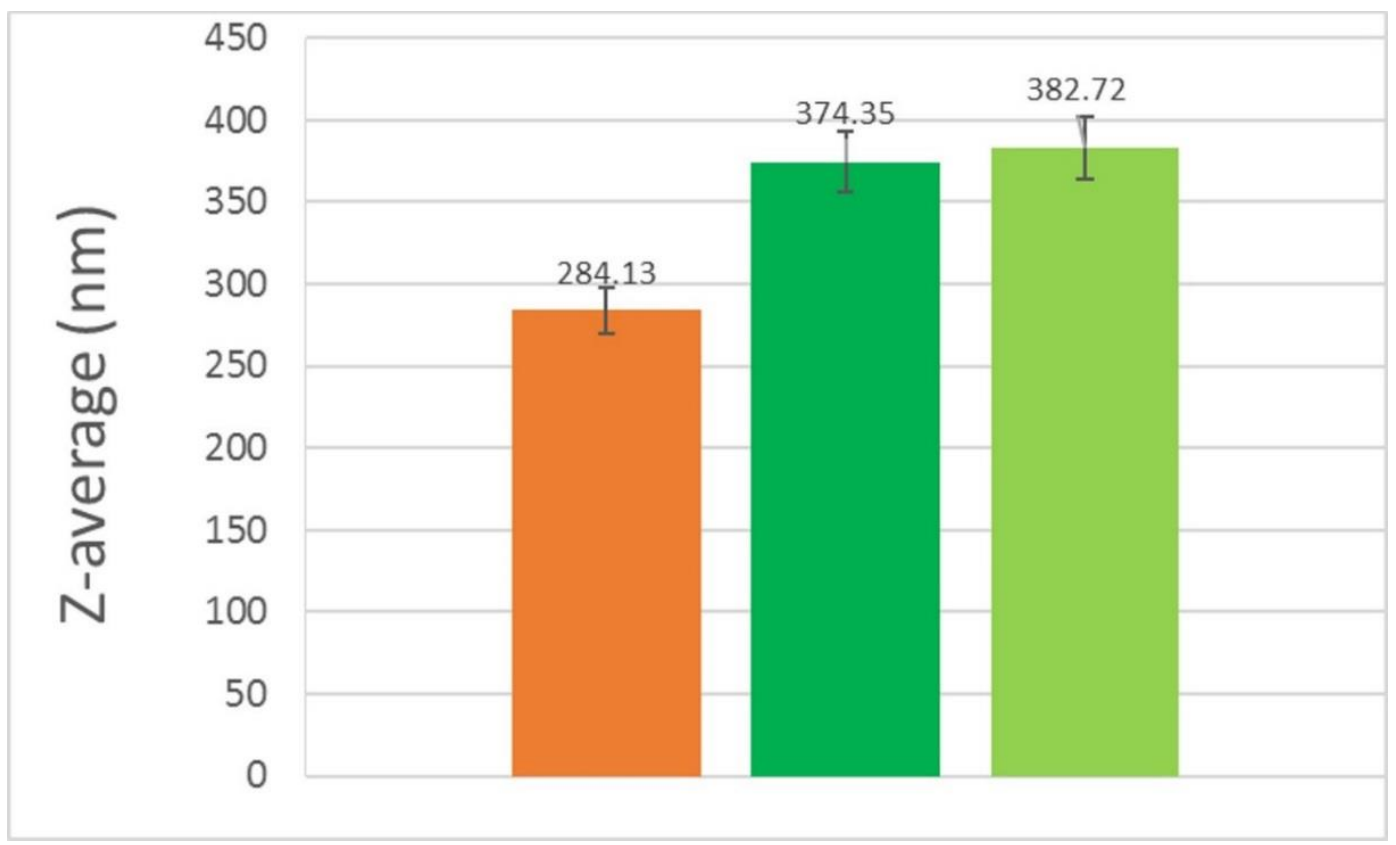

(B)

Figure 18. (from left to the right)

Main particle size of nanoMel (measured by laser diffraction), fluidMel fresh and fluidMel stored (6 months) (measured by SEM images) (A) and Z-average of nanoMel, lyoMel fresh, and lyoMel stored (6 months) measured by Zeta nano ZS) (B) 


\subsubsection{SEM measurements}

Figure 19A and Figure 19B clearly show the particle size difference between the rawMel and nanoMel and the change in the particle habit. The latter particles have smooth surfaces with rounded edges and corners. High mechanical impact results in the fracture and abrasion of the crystals.
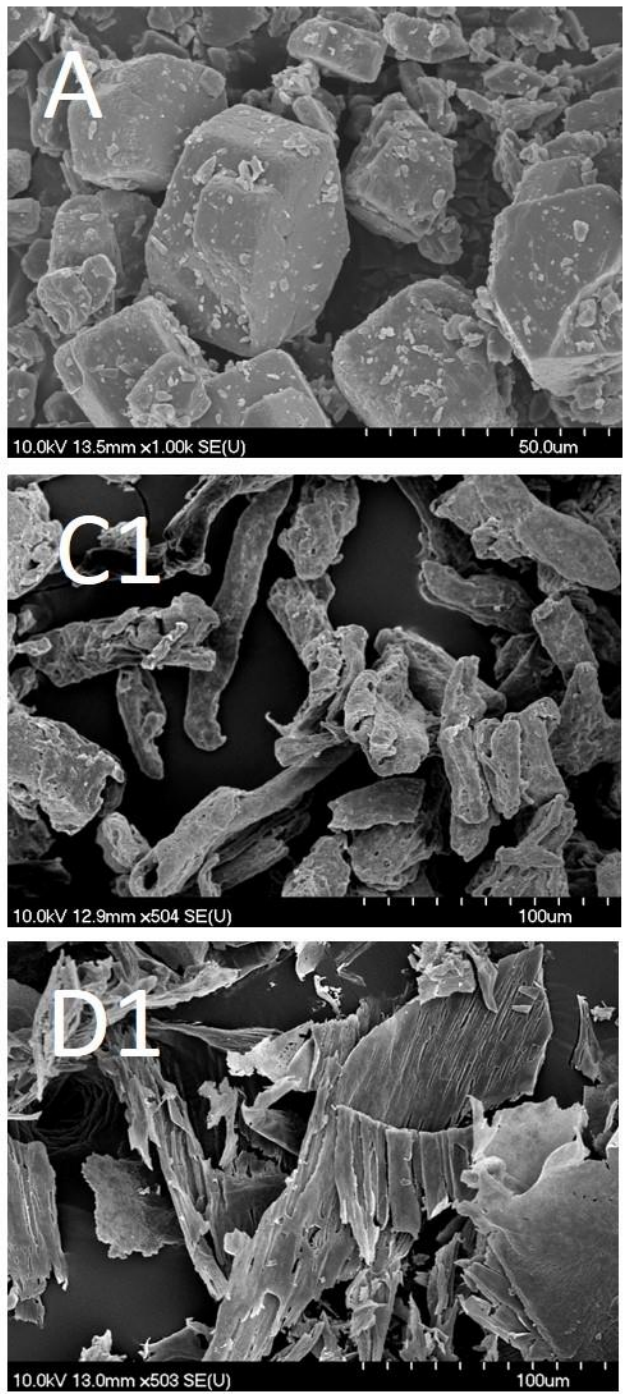
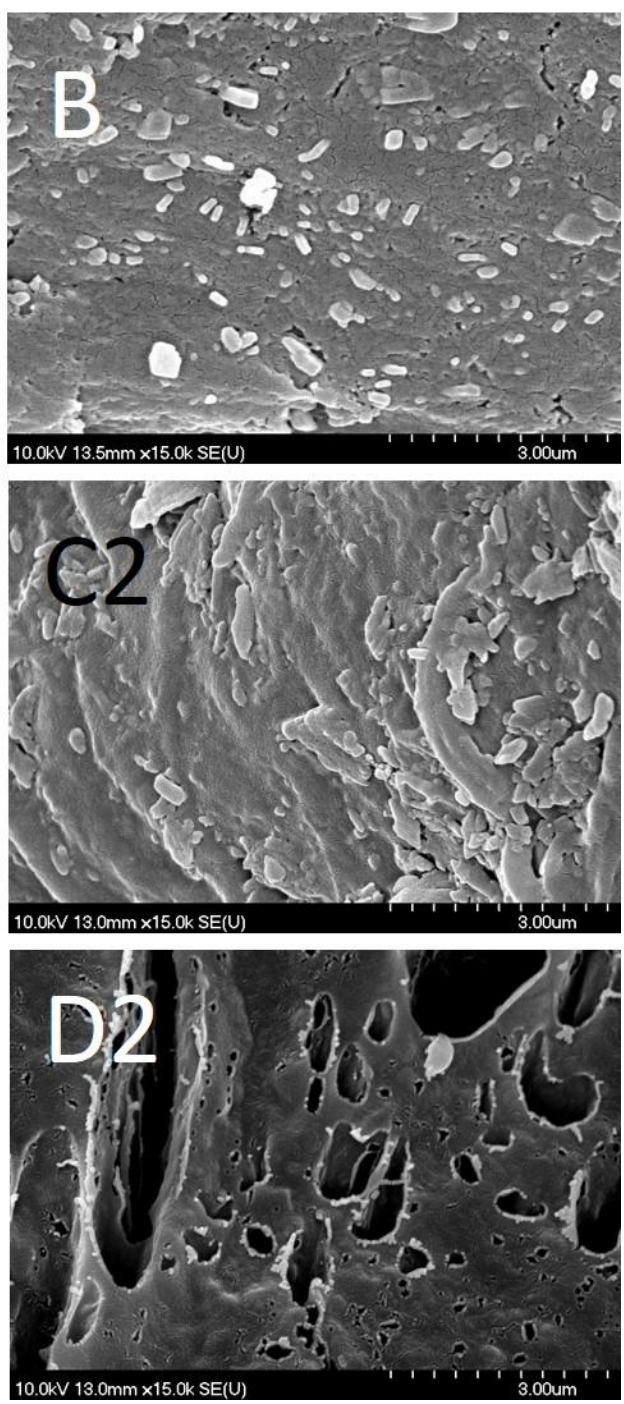

Figure 19. SEM image of rawMel (A), nanoMel (B), fluidMel (C1 and C2) and lyoMel (D1 and D2)

During the fluidization process, the nanoparticles are uniformly adhered to the surface of MCC (Figure 19C1). Their habit is the same as that of the nanoparticles in the nanoMel (Figure 19C2). There is no sign indicating the aggregation of the nanoparticles on the surface of MCC. The adhesion of the Mel particles to the carrier surface is also supported by the effect of the PVA adhesive property and the rapid evaporation of water. 
The SEM image of the lyoMel sample (Figure 19D1) shows large, consistent formulas at low magnification. The texture at higher magnification contains smaller, larger pores resulting in a big surface with honeycombed structure, where the surface area is determined by the size of the ice crystals (Kevin, 2018). The SEM picture does not show any trehalose-like crystals in the structure (Figure 19D2).

\subsubsection{DSC measurements, crystallinity determination}

The DSC curves of the components and the products are shown in Figure 3. The rawMel has a relative high melting point at $268^{\circ} \mathrm{C}$, PVA as semi-crystalline material has two endothermic peaks at $169^{\circ} \mathrm{C}$ and at $222^{\circ} \mathrm{C}$. MCC shows any characteristic peaks, in contrast the trehalose is a crystalline material (Figure 20A).

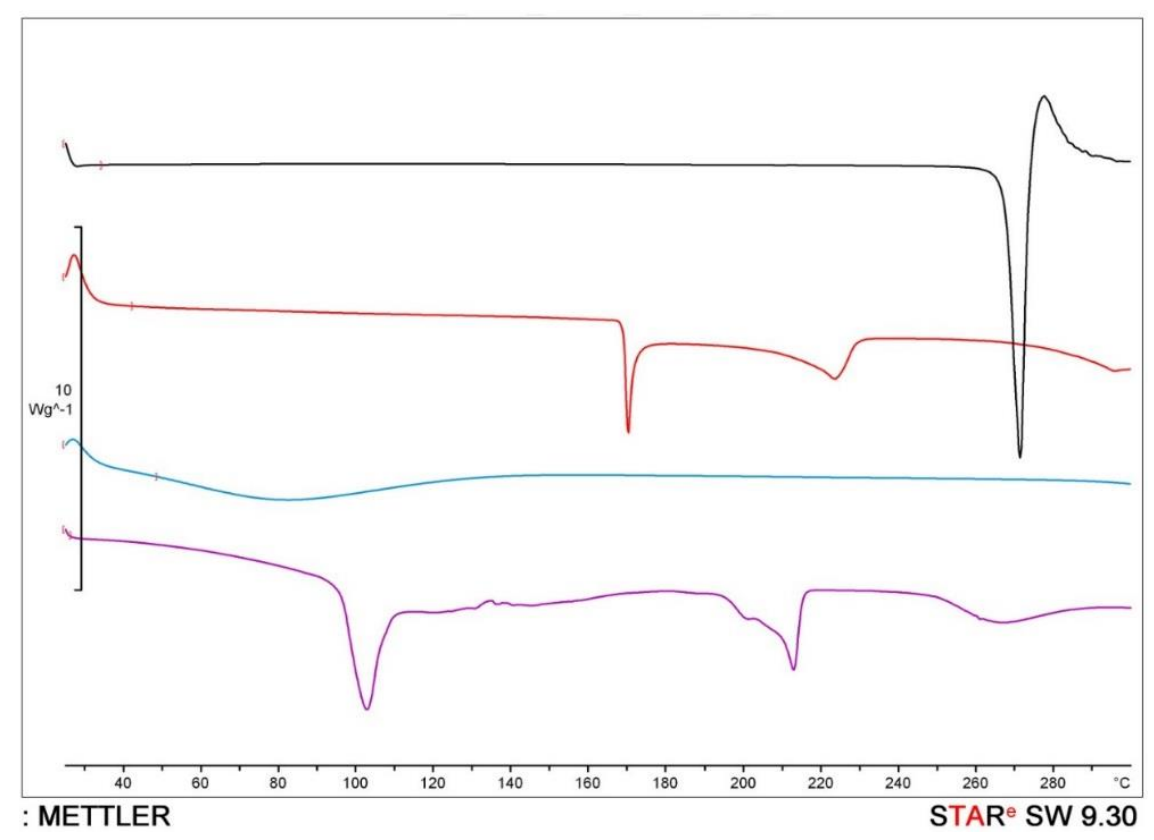




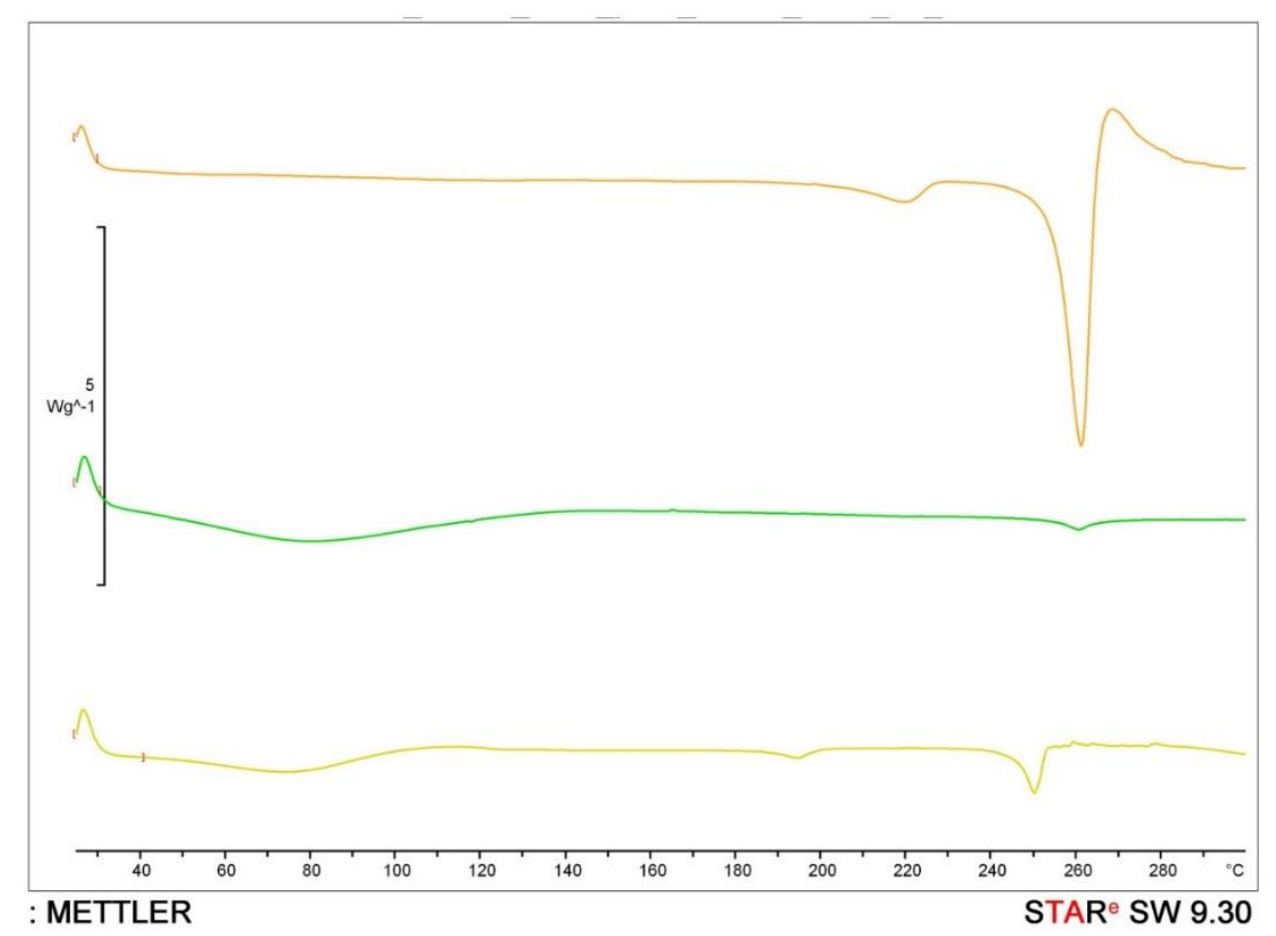

Figure 20. DSC curves of rawMel (black), PVA (red), MCC (blue) and trehalose (purple) (A), and nanoMel (orange) fluidMel (green) and lyoMel (yellow) (B)

The thermograms of the products are shown in Figure 20B. It is clear, that the melting point and the enthalpy of Mel in the case of the nanoMel decreased due to the partial amorphization.

The curve of the fluidMel shows the peak of MCC and the decreased melting point of Mel (Figure 20B). The first obvious and big endothermic peak of the curve appearing from $30{ }^{\circ} \mathrm{C}$ to $150{ }^{\circ} \mathrm{C}$ is mainly related to the absorbed moisture evaporation (Kian et al., 2017). The second endothermic peak is connected to the melting point of $\mathrm{Mel}\left(264{ }^{\circ} \mathrm{C}\right)$ and the enthalpy was decreased due to the large amount of MCC, which covered the characteristic peak of PVA as well, compared to nanoMel.

The curve of lyoMel represents the peak of PVA $\left(197^{\circ} \mathrm{C}\right)$ and the melting temperature of Mel $\left(251{ }^{\circ} \mathrm{C}\right)$ (Figure 20B). According to the literature (Sussich et al, 2008) and our measurements, during the process, the total amount of trehalose transformed into an amorphous form and the lyophilized trehalose maintains its amorphous form. The big endothermic peak of the curve appearing from $30{ }^{\circ} \mathrm{C}$ to $150{ }^{\circ} \mathrm{C}$ is connected to the absorbed water evaporation as well. 
The crystallinity of Mel in nanoMel and the transformed solid-state products was calculated by the enthalpy changes of the drug occurring during the DSC measurement (Table 11). Each sample was compared to its own physical mixture. According to the crystallinity of the nanoMel $(13.43 \%)$ sample, the crystallinity of the fluidMel sample did not change (12.98 \%), for the lyoMel sample partial recrystallization (40.11\%) occurred.

Table 11. Enthalpy and calculated crystallinity values of the characteristic peak of Mel in the samples

\begin{tabular}{|l|c|c|c|}
\hline Sample & $\begin{array}{c}\text { Enthalpy } \\
(\mathrm{J} / \mathrm{g})\end{array}$ & $\begin{array}{c}\text { Crystallinity of Mel } \\
(\%)\end{array}$ & $\begin{array}{c}\text { Crystallinity of Mel } \\
\text { after 6 months of } \\
\text { storage (\%) }\end{array}$ \\
\hline nanoMel & 12.24 & 13.43 & - \\
\hline fluidMel & 11.83 & 12.98 & 13.02 \\
\hline lyoMel & 36.54 & 40.11 & 40.16 \\
\hline
\end{tabular}

After 6 months of storage $\left(23 \pm 2{ }^{\circ} \mathrm{C}, 45 \pm 5 \% \mathrm{RH}\right)$, the degree of crystallinity of solidified samples (fluidMel and lyoMel) was determined again. The results did not show a significant change ( $p>0.05)$ compared to the non-stored, fresh samples (Table 10). There was no sign for the recrystallization of trehalose.

\subsubsection{Drug content determination}

The theoretical drug content was $7.50 \mathrm{mg}$ as single dose/oral. For the nanoMel sample this amount was $7.12 \mathrm{mg}$ and fluidMel showed $6.83 \mathrm{mg}$ of Mel. The latter can be related to the yield of the fluidization technique (95.93\%). During the lyophilization process the Mel content of the sample (lyoMel) was $7.12 \mathrm{mg}$.

\subsubsection{Solubility testing of MEL in the samples}

The solubility of nanoMel increased significantly $(9.4 \pm 0.5 \mu \mathrm{g} / \mathrm{ml})$ in comparison with the rawMel $(6.5 \pm 0.2 \mu \mathrm{g} / \mathrm{ml})$. The reduced particle size enhanced the wettability of the hydrophobic particle when using PVA, therefore increased the thermodynamic solubility of Mel. The fluidization process did not affect the solubility of Mel $(9.6 \pm 0.4 \mu \mathrm{g} / \mathrm{ml})$. In the case of lyoMel, solubility was increased $(11.2 \pm 0.5 \mu \mathrm{g} / \mathrm{ml})$ because of the presence of trehalose. 


\subsubsection{In vitro dissolution studies}

The in vitro dissolution extent of the samples was investigated in gastric juice $(\mathrm{pH}=1.2)$. Mel has a week acidic character, therefore its solubility in this medium is very poor $(1.6 \pm 0.2$ $\mu \mathrm{g} / \mathrm{ml}$, at $37^{\circ} \mathrm{C}$ ). Figure 21 clearly demonstrates that the particle size reduction of $\mathrm{Mel}$ in the nanosuspension (nanoMel) influenced the dissolution rate of Mel, but resulted in only $40 \%$ of drug release in 5 minutes, and then the curve took a stagnant profile. The initial rapid drug release can be associated with the nanoscale Mel and its amorphous structure. The 2-hour test did not result in any more favorable results. Although the distribution of the nanoparticles of Mel in the nanosuspension is suitable, a large volume of acidic medium $(900 \mathrm{ml})$ may increase the aggregation of the nanoparticles. In this case, the protective effect of the polymer (PVA) is unsatisfactory.

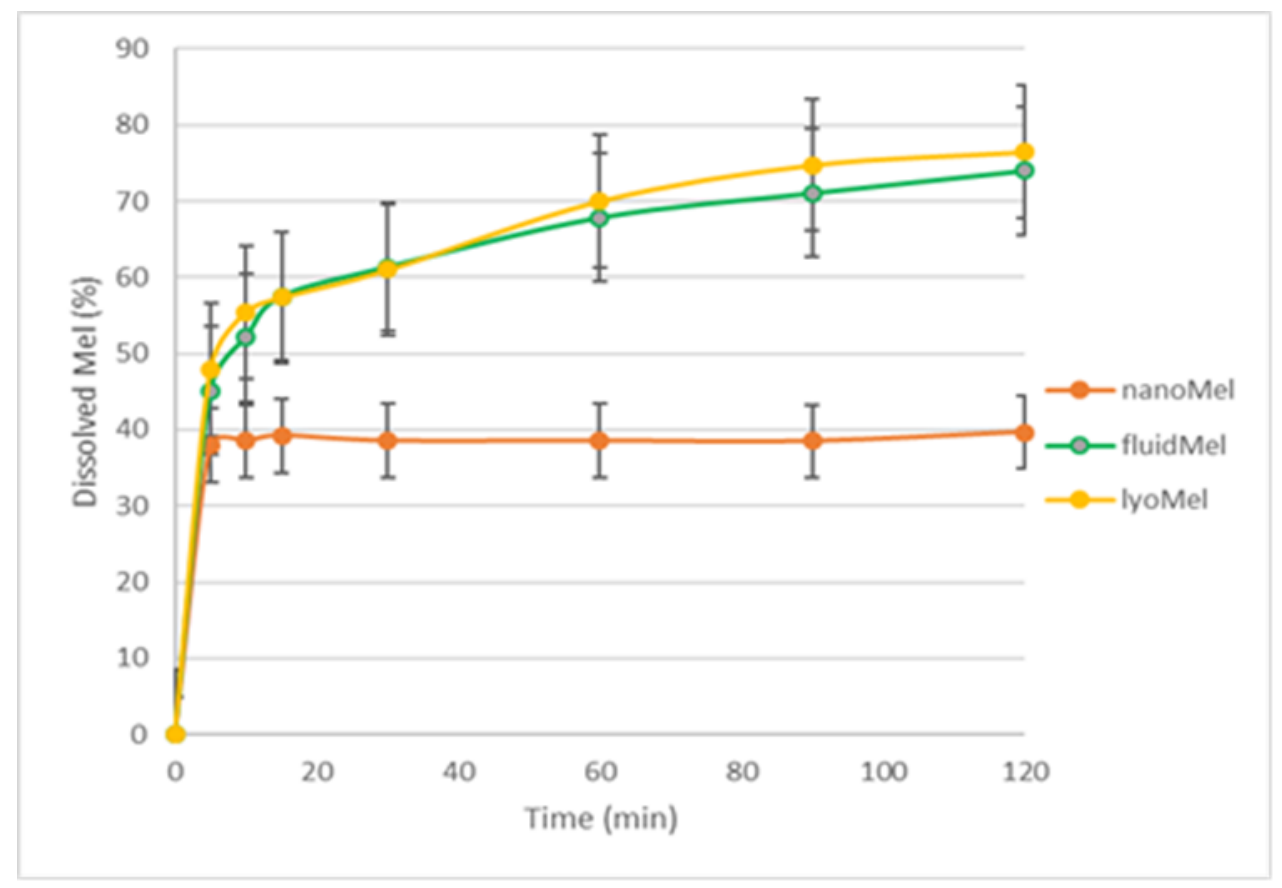

Figure 21. In vitro dissolution of Mel from investigated samples. Medium: artificial gastric juice (pH: 1.2)

For fluidMel and lyoMel samples, a rapid initial phase is observed (about $60 \%$ of the drug is dissolved in 15 minutes), followed by a slowing but rising profile. About $75 \%$ of Mel was dissolved within 2 hours. In the case of the solid-state forms, carriers (MCC and trehalose) help to uniformly distribute the nanoparticles of Mel, thereby maintaining the uniqueness of the nanoparticles. 


\subsubsection{In vivo studies}

The plasma concentration of the samples in rats is shown in Figure 22. The calculated plasma concentration of Mel at zero min $\left(\mathrm{C}_{0 \mathrm{~min}}\right)$ was $10,607 \mathrm{nM}$, and then the plasma concentration decreased exponentially. A very small amount of Mel was absorbed from nanoMel sample, regardless of the particle size of the drug. The plasma concentration of Mel was constant in the investigated time period. The results show that the nanosuspension (nanoMel) has not got advantageous properties.

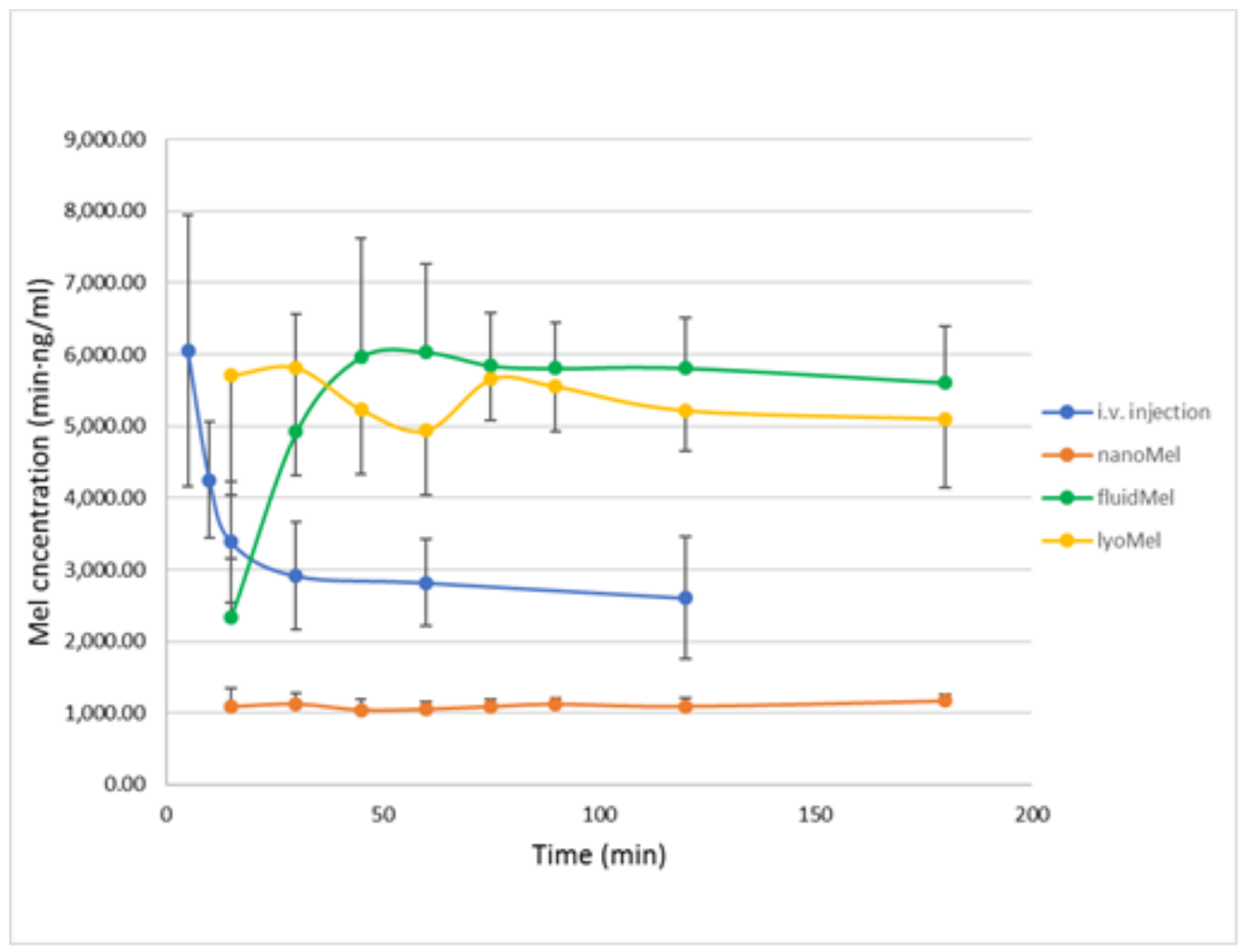

Figure 22. Plasma levels of MEL after the administration of different samples in rats. The preparations were administered orally (nanoMel, fluidMel and lyoMel) or intravenously (IV) as a single dose of $300 \mu \mathrm{g} / \mathrm{kg}$.

The initial blood levels of the fluidMel and lyoMel samples show a big difference. At 15 minutes, the lyoMel sample $(5,712.98 \mathrm{nM})$ shows more than twice the value of fluidMel (Table 11). In practice, this value is similar as the maximum plasma level for the lyophilized product $\left(\mathrm{C}_{30 \mathrm{~min}} 5,814 \mathrm{nM}\right)$. The peak blood concentration of fluidMel, is about $6,000.00 \mathrm{nM}$ at 50 minutes that is comparable with the blood concentration reached by IV injection at 5 min. This result also confirms that the solid products contained Mel in an adequate amount, and that the total amount thereof dissolved and absorbed. 
The plasma curves of the different samples containing Mel show a very slow elimination after the distribution phases. That can be explained by the very high (99\%) plasma binding property of Mel in rat, and this ratio is the same in human (Busch et al., 1998). It seems that the eliminated portion of $\mathrm{Mel}$ is replenished from the protein bounded fraction for a quite long period of time. Our measuring time was only 3 hours, longer detection period can provide appropriate information about the whole elimination process. The peak MEL concentrations of from lyoMel and fluidMel preparations have reached the similar level that of IV formula (Figure 22). The lyoMel sample resulted in higher plasma concentrations in 15 minutes as compared with nanoMEL preparation. The solidified samples had nearly five-fold higher bioavailability than that of nanoMel (Table 12).

Table 12. Plasma concentrations of $\mathrm{Mel}$ in time and its relative bioavailability in rats after IV and per os administration of Mel samples. Relative bioavailabilities were compared to nanoMel preparation

\begin{tabular}{|l|l|l|l|l|}
\hline Sample & $\begin{array}{l}\mathrm{C}_{15 \min } \\
(\mathrm{nM})\end{array}$ & $\begin{array}{l}\mathrm{C}_{120 \mathrm{~min}} \\
(\mathrm{nM})\end{array}$ & $\begin{array}{l}\text { AUC } \\
(\mathrm{min} \cdot \mathrm{ng} / \mathrm{ml})\end{array}$ & $\begin{array}{l}\text { Relative } \\
\text { bioavailability } \\
(\%)\end{array}$ \\
\hline nanoMel & $1,090.02$ & $1,123.31$ & $190,584.52$ & 100.00 \\
\hline fluidMel & $2,338.44$ & $5,811.33$ & $945,834.99$ & 496.28 \\
\hline lyoMel & $5,712.98$ & $5,219.52$ & $923,117.95$ & 484.36 \\
\hline IV injection & $\mathrm{C}_{5 \min } 6,059.07$ & $2,607.80$ & $377,528.01$ & - \\
\hline
\end{tabular}

\subsubsection{IVIV correlation}

Comparative studies according to AUC values have shown that there are significant differences between the nanosuspension and the samples (fluidMel and lyoMel) within in vitro and in vivo groups. However, there is no significant difference between two solid samples either in vitro or in vivo (Figure 23). The basis of the IVIVC calculation was the comparison of the AUC values of the samples in the in vitro and in vivo groups. By our calculations, the Pearson's correlation coefficient value between the two studies is 0.99695 . The $\mathrm{t}$ value of the independent t-test of the two dissolution study series was 0.0145 , the 
calculated p value was 0.9889 and the difference is not significant at a confidence level of 95\%. As the zero hypothesis of the independent t-test, the calculation is not significant if the averages of the two series are equal. It can be concluded that in this system, in vitro dissolution studies are applicable to predict the dissolution rate-limited differences in the case of in vivo studies.

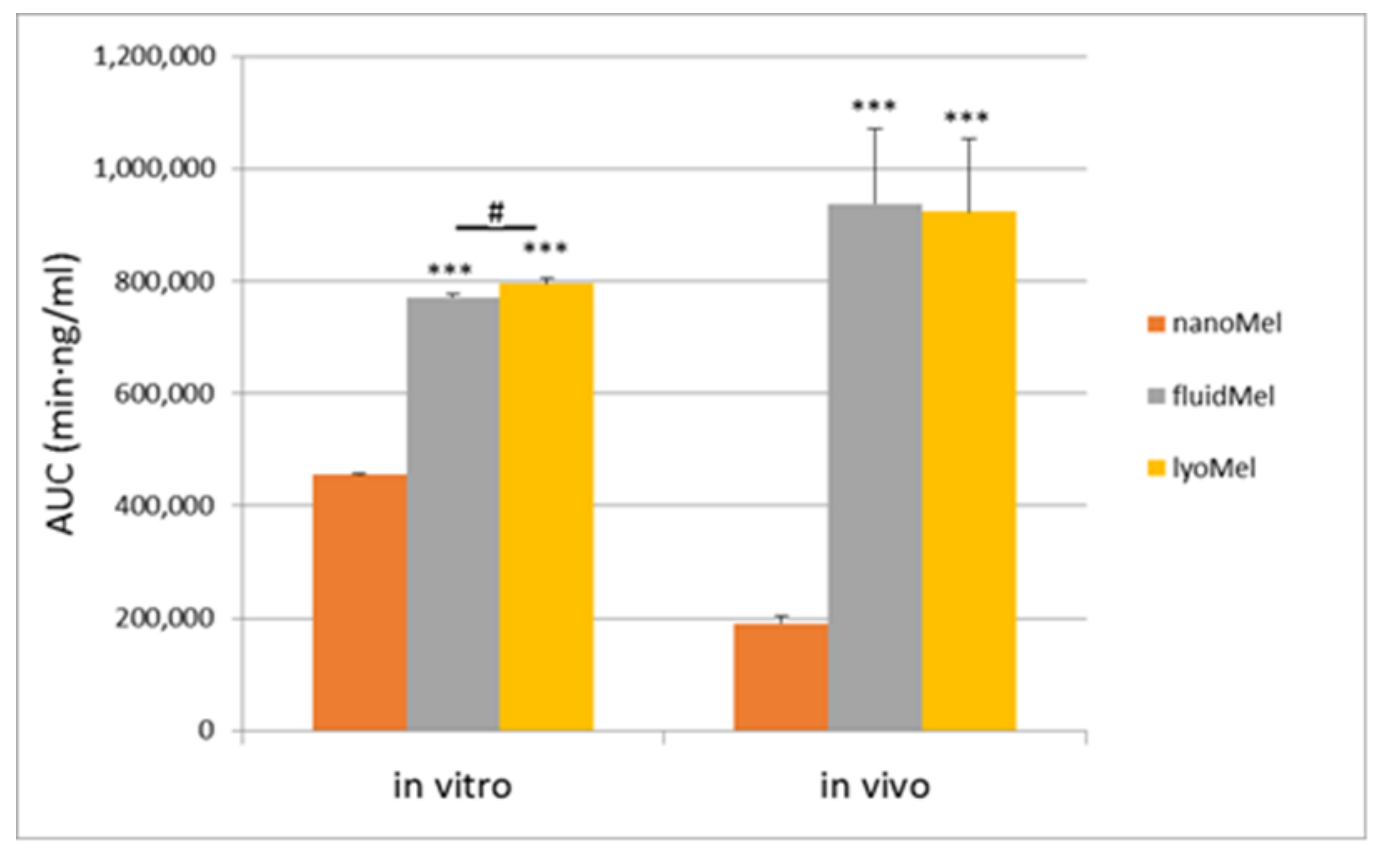

Figure 23. IVIV correlation of Mel containing samples. Notes: Values are presented as mean \pm SD. Statistically significant differences are: $* * * p<0.001$, compared to nanoMel separately in in vitro and in vivo groups; \# $\mathrm{p}<0.05$ compared to the indicated columns

\section{CONCLUSION}

i) The PhD work reports a wet milling process, where the planetary ball mill was combined with pearl milling technology to produce nano-size meloxicam $(\mathrm{Mel})(\mathrm{d}(0.9)<500 \mathrm{~nm})$. Mel as a water-insoluble highly potent NSAID was milled in the presence of the PVA, in aqueous solution any other stabilizer agent as surfactant. The critical process parameters (ratio of predispersion and pearls, milling time and rotation speed of the steel jar) were optimized by factorial design on the particle size distribution and crystallinity of the Mel were investigated. It was found that the ratio of pre-dispersion and pearls 1:1 (w/w) resulted in the most effective grinding system without any pre-milled process (200-fold particle size reduction in one step) with $437 \mathrm{rpm}$ and $43 \mathrm{~min}$ as optimized parameters.

ii) The amount of PVA were also a critical parameter because it affected the milling effectiveness, the particle size distribution and the crystallinity of Mel. The different 
concentrations of PVA in the aqueous dispersion influenced the viscosity and the electrokinetic property of the particle, and the stability of the dispersions. It was found that the milling effectiveness of low concentration of PVA $(<4 \%)$ was not satisfactory, because the crushing / breaking effect of the pearls could less prevail. High concentration of PVA (> 5\%) also resulted in unsatisfactory milling effectiveness, because of the formation of polymer layer on surface of particles which protects the particles from the fragmentation. Considering the effectiveness of milling, 5\% PVA was proved to be an optimal quantity to meet the expected value $(\mathrm{d}(0.9)<500 \mathrm{~nm})$. Finally, the sample (diluted dispersion) containing $1 \%$ Mel and $0.5 \%$ PVA produced by the optimized wet milling procedure fulfilled the requirements for the nanosuspension with respect to particle size distribution $(\mathrm{d}(0.1)$ $0.067 \mu \mathrm{m}, \mathrm{d}(0.5) 0.130 \mu \mathrm{m}, \mathrm{d}(0.9) 0.371 \mu \mathrm{m})$. The intermediate product showed a stable system with 2 weeks of holding time.

iii) In vitro dissolution tests have shown that the particle size of the Mel and its degree of crystallinity are interdependent critical parameters, which plays an important role in the fast drug release. The human Caco-2 cell culture studies justified that the penetration of Mel from different PVA-containing products was significantly increased as compared to Mel suspension without toxic effects. From all the tested samples, the $\mathrm{P}_{\text {app }}$ value of Mel was the highest in the investigated sample containing $0.05 \%$ PVA, which belongs to the optimized nanosuspension.

iv) The further aim of this work was to discover the robustness of combined wet milling process to determine the interval of the Mel amount and to predict the degree of crystallinity of the milled samples as critical parameter using DSC and XRPD measurements. The samples had the PVA as the stabilizing agent. By increasing the amount of the Mel, its crystallinity increased, and close correlation was found between the degree of crystallinity and the Mel amount. To achieve the desired particle size $(<500 \mathrm{~nm})$, the Mel amount should be changed between 10.0 and $17.5 \%(\mathrm{w} / \mathrm{w})$ and a PVA concentration should be used between 5.0 and $4.58 \%(\mathrm{w} / \mathrm{w})$. In this specified range, the degree of crystallinity of Mel will be changed between 20 and $45 \%$. The crystallinity of Mel investigated by DSC and XRPD did not show any significant difference at 95\% significance level.

v) Mel containing (1\%) nanoMel sample produced by wet milling process using optimized amount of PVA $(0.5 \%)$ resulted in $130 \pm 5 \mathrm{~nm}$ as mean particle size and a significant reduction in the degree of crystallinity (13.43\%) of Mel. The fluidization technique using microcrystalline cellulose (MCC) as carrier resulted a quick conversion no significant change in the critical product parameters. Process of lyophilization required a longer operation time, 
which resulted in the amorphization of the crystalline carrier (trehalose) and the recrystallization of Mel increased its particle size and crystallinity. In accordance with this, the particle size (Z-average: from $284 \mathrm{~nm}$ to $374 \mathrm{~nm}$ ) and the degree of crystallinity of Mel (to $36.54 \%$ ) were also changed. The 6-month storage did not cause any further changes in the products.

vi) To justify applicability of surfactant-free samples containing nanonized Mel in vivo studies was performed. The nanonized Mel in solidified products (fluidMel, lyoMel) resulted in rapid absorption through the gastric membrane by passive transcellular transport. It was found that these products contained Mel in an adequate amount, and that the total amount thereof dissolved and absorbed. The fluidMel and lyoMel samples had nearly five-fold higher relative bioavailability than nanoMel application by oral administration. The correlation between in vitro and in vivo studies showed that the fixed Mel nanoparticles on the surface of solid carriers (MCC, trehalose) in both the artificial gastric juice and the stomach of the animals rapidly reached saturation concentration leading to faster dissolution and rapid absorption.

\section{New findings of this work:}

Combined wet milling is considered to be a suitable process for nanonization of active ingredients, since it produces a desired particle size product in a single step without using premilling and surfactant (innovative technology). The novelty of the research results is the determination and optimization of critical process and product parameters, which is well demonstrated by the production of Mel containing nanosuspension (nanoMel).

To discover the robustness of the milling process, it should also be considered that the amount of grinding media can be reduced by increasing the amount of the active ingredient and the crystallinity of the drug can be regulated. In this case, the DSC method can be suggested for the quantification of the degree of crystallinity because it can be used safely with high amorphous content.

The solidification of the nanosuspension not only increases the stability of the nanoparticles (particle size, crystallinity degree), but also allowes the preparation of surfactant-free solid compositions (powder, tablet, capsule) with excellent bioavailability, which may be an important consideration for certain groups of patients to achieve rapid analgesia. Further experiments are necessary to prove the therapeutic relevance of these formulations (innovative product). 


\section{REFERENCES}

Abramoff MD, Magelhaes PJ, Ram SJ. Image processing with image. J. Biophotonics Int. 11 (2004) 36-42.

Azad M, Afolabi A, Patel N, Davé R, Bilgili E. Preparation of stable colloidal suspensions of superdisintegrants viawet stirred media milling. Particuology 14 (2014) 76-82.

Azad M, Afolabi A, Bhakay A, et al. Enhanced physical stabilization of fenofibrate nanosuspensions via wet co-milling with a superdisintegrant and an adsorbing polymer. Eur. J. Pharm. Biopharm. 94 (2015) 372-385.

Austin L G. A Commentary on the Kick, Bond and Rittinger Laws of Grinding. Powder Technol. 7 (1973) 315-317.

Baláž P. Mechanochemistry in Minerals Engineering. In: Mechanochemistry in Nanoscience and Minerals Engineering. Springer, Berlin, Heidelberg (2008).

Bartos C, Kukovecz Á, Ambrus R, Farkas G, Radacsi N, Szabó-Révész P. Comparison of static and dynamic sonication as process intensification for particle size reduction using a factorial design. Chem. Eng. Proces.: Process Intensification 87 (2015) 26-34.

Bartos C, Ambrus R, Sipos P, Budai-Szücs M, Csányi E, Róbert Gáspár ${ }^{c}$, Árpád Márkic, Adrienn B. Seres ${ }^{c}$, Anita Sztojkov-Ivanov ${ }^{c}$, Tamás Horváth ${ }^{a}$, Piroska Szabó-Révész, Study of sodium hyaluronate-based intranasal formulations containing micro- or nanosized meloxicam particles, Int. J. Pharm. 491 (2015) 198-207.

Bartos C, Szabó-Révész P, Bartos Cs, Katona G, Jójárt-Laczkovich O, Ambrus R. The Effect of an Optimized Wet Milling Technology on the Crystallinity, Morphology and Dissolution Properties of Micro- and Nanonized Meloxicam. Molecules 21 (2016) 507-518.

Bartos Cs, Jójárt-Laczkovich O, Katona G, Budai-Szücs M, Ambrus R, Bocsik A, Gróf I, Deli M A, Szabó-Révész P. Optimization of a combined wet milling process in order to produce poly(vinyl alcohol) stabilized nanosuspension. Drug. Des. Dev. Ther. 12 (2018) 1567-1580.

Bartos C, Pallagi E, Szabó-Révész P, Ambrus R, Katona G, Kiss T, Rahimi M, Csóka I. Formulation of levodopa containing dry powder for nasal delivery applying the quality-bydesign approach. Eur. J. Pharm. Sci. 123 (2018) 475-483.

Bartos Cs, Jójárt-Laczkovich O, Regdon G Jr, Szabó-Révész P. Robustness testing of milling process, analyzing the particle size distribution and crystallinity of the milled samples. $J$. Therm. Anal. Cal. (2019) DOI: 10.1007/s10973-019-08395-2

Bartos Cs, Ambrus R, Katona G, Gáspár R, Márki Á, Ducza E, Ivanov A, Tömösi F, Janáky T, Szabó-Révész P. Transformation of meloxicam containing nanosuspension into surfactantfree solid compositions to increase the product stability and drug bioavailability for rapid analgesia. Drug Des. Dev. Ther. (2019 accepted for publication) 
Bhakay A, Azad M, Vizzotti E, Dave R N, Bigili E. Enhanced recovery and dissolution of griseofulvin nanoparticles from surfactant-free nanocomposite microparticles incorporating wet-milled swellable dispersants. Drug Dev. Ind. Pharm. 40 (2014) 1509-1522.

Bhatia A, Chopra S, Nagpal K, Deb P K, Tekade M, Tekade R. Chapter 2 - Polymorphism and its Implications in Pharmaceutical Product Development. Dosage Form Design Parameters volume 2 (2018) 31-65.

Bilgili E, Hamey B, Scarlett B. Nano-milling of pigment agglomerates using a wet stirred media mill: Elucidation of the kinetics and breakage mechanisms. Chem. Eng. Sci. 61 (2006) $149-157$.

Bose S, Schenck D, Ghosh I, Hollywood A, Maulit E, Ruegger C. Application of spray granulation for conversion of a nanosuspension into a dry powder form. Eur. J. Pharm. Sci. 47 (2012) 35-43.

Broseghini M, Gelisio L, Incau M D, Azanza Ricardo C N, Pugno N M, Scardi P. Modeling of the planetary ball-milling process: The case study of ceramic powders. J. Eur. Ceram. Soc. 36 (2016) 2205-2212.

Busch U, Schmid J, Heinzel G, Schmaus H, Baierl J, Huber C, Roth W. Pharmacokinetics of Meloxicam in Animals and The Relevance to Humans. Drug. Metab. Dispos. 26 (1998) 576584.

Chan H K, Kwok P C L. Production methods for nanodrug particles using the bottom-up approach. Adv. Drug Deliv. Rev. 63 (2011) 406-416.

Chiang P C, Wahlstrom J L, Selbo J G, Zhou S, Wene S P, Albin L A, Warren C J, Smith M E, Roberds S L, Ghosh S, Zhang L L, Pretzer D K. 2007. 1,3- Dicyclohexyl urea nanosuspension for intravenous steady-state delivery in rats. J. Exp. Nanosci. 2 (2007) 239250.

Colombo M, Orthmann S, Bellini M et al. Influence of drug brittleness, nanomilling time, and freeze-drying on the crystallinity of poorly water-soluble drugs and its implications for solubility enhancement. AAPS Pharm. Sci. Tech. 18 (2017) 2437-2445.

Dahiya s. Drug Nanonization: An Overview Of Industrially Feasible Top-Down Technologies For Nanocrystal Production. B. Pharm. Sci. 7 (2017) 144-152.

Dávid Á, Benkóczy Z, Ács Z, Greskovits D, Dávid Á Z. The theoretical basis for scaling-up by the use of the method of microwave granulation. Drug Dev. Ind. Pharm. 26 (2000) 943951.

De Merlis C C, Schoneker D R. Review of the oral toxicity of polyvinyl alcohol (PVA), Food Chem. Toxicol. 41 (2003) 319-326. 
Derjaguin B V, Landau L. Theory of the stability of strongly charged lyophobic sols and of the adhesion of strongly charged particles in solutions of electrolytes. Acta Phys Chim 14 (1941) 633-662.

Ding Z, Wang L, Xing Y, Zhao Y, Wang Z, Han J. Enhanced Oral Bioavailability of Celecoxib Nanocrystalline Solid Dispersion based on Wet Media Milling Technique: Formulation, Optimization and In Vitro/In Vivo Evaluation. Pharmaceutics 11 (2019) 328346.

Dokoumetzidis A, Macheras P. A century of dissolution research: From Noyes and Whitney to the Biopharmaceutics Classification System. Int. J. Pharm. 321 (2006) 1-11.

Duroudier J P. Ball and Rod Mills. Size Reduction of Divided Solids (2016) 73-97.

Finke J H, Juhnke M, Kwade A, Bunjes H. Overview of of techniques and description of established processes. Pharmaceutical Nanotechnology, 2 Volumes: Innovation and Production, Jean Cornier, Andrew Owen, Arno Kwade, Marcel Van de Voorde. John Wiley and Sons (2017).

Fülöp V, Jakab G, Bozó T, Tóth B, Endrésik D, Kellermayer M, Balogh E, Antal I. Study on the dissolution improvement of albendazole using reconstitutable dry nanosuspension $\begin{array}{lllllll}\text { formulation. Eur. } & \text { J. Pharm. Sci. } & 123 & \text { (2018), } & \text { 70-78. }\end{array}$ https://doi.org/10.1016/j.ejps.2018.07.027

Gao M, Forssberg E. Prediction of product size distributions for a stirred ball mill. Powder Technol. 84 (1995) 101-106.

Garcia-Cerda L A, Escareno-Castro M U, Salazar-Zertuche M. Preparation and characterization of polyvinyl alcohol-cobalt ferrite nanocomposites. J. Noncryst. Solids. 353 (2007) 808-810.

George M, Ghosh I. Identifying the correlation between drug/stabilizer properties and critical quality attributes (CQAs) of nanosuspension formulation prepared by wet media milling technology. Eur. J. Pharm. Sci. 48 (2013) 142-152.

Ghosh I, Schenck D, Bose S, Ruegger C. Optimization of formulation and process parameters for the production of nanosuspension by wet media milling technique: Effect of Vitamin $\mathrm{E}$ TPGS and nanocrystal particle size on oral absorption. Eur. J. Pharm. Sci. 47 (2012) 718 728.

Gieszinger P, Csóka I, Pallagi E, Katona G, Jójárt-Laczkovich O, Szabó-Révész P, Ambrus R. Preliminary study of nanonized lamotrigine containing products for nasal powder formulation. Drug. Des. Dev. Ther. 11 (2017) 2453-2466.

Girdhar V, Patil S, Banerjee S, Singhvi G. Nanocarriers For Drug Delivery: Mini Review. Recent Pat. Nanomed. 8 (2018) 88-99. 
Gombás Á, Szabó-Révész P, Kata M, Regdon G Jr., Erős I. Quantitative determination of crystallinity of a lactose monohydrate by DSC. J. Therm. Anal. Calorim. 68 (2002) 503-510.

He W, Lu Y, Qi J, et al. Formulating food protein-stabilized indomethacin nanosuspensions into pellets by fluid-bed coating technology: physical characterization, redispersibility, and dissolution. Int J Nanomedicine. 8 (2013) 3119- 3128.

Harris K D M. Mechanochemical synthesis: how grinding evolves. Nat. Chem. 5 (2013) $12-$ 14.

Heegn $\mathrm{H}$. The influence of the fine grinding on thestructure and energy content of solids. Proceedings of $6^{\text {th }}$ POWTECH (1979) 61-69.

Hellinger E, Veszelka S, Tóth AE, Walter F, Kittel A, Bakk ML, Tihanyi K, Háda V, Nakagawa S, Duy TD, Niwa M, Deli MA, Vastag M. Comparison of brain capillary endothelial cell-based and epithelial (MDCK-MDR1, Caco-2, and VB-Caco-2) cell-based surrogate blood-brain barrier penetration models. Eur. J. Pharm. Biopharm. 83 (2012) 340351.

Horváth T, Ambrus R, Völgyi G, Budai-Szücs M, Márki Á, Sipos P, Bartos C, B.Seres A, Sztojkov-Ivanov A, Takács-Novák K, Csányi E, Gáspár R, Szabó-Révész P. Effect of solubility enhancement on nasal absorption of meloxicam. Eur. J. Pharm. Sci. 95 (2016) 96102.

Hui Loh Z, Kumar Samanta A, Wan Sia Heng P. Overview of milling techniques for improving the solubility of poorly water-soluble drugs. Asian J. Pharm. Sci. 10 (2015) 255274.

Jia L, Wong H, Cerna C, Weitman S D. Effect of nanonization on absorption of 301029: ex vivo and in vivo pharmacokinetic correlations determined by liquid chromatography/mass spectrometry. Pharm. Res. 19 (2002) 1091-1096.

Jójárt-Laczkovich O, Szabó-Révész P. Formulation of tablets containing an 'in-process' amorphized active pharmaceutical ingredient. Drug. Dev. Int. Pharm. 37 (2011) 1272-1281.

Junghanns J U A H, Müller R H. Nanocrystal technology, drug delivery and clinical applications. Int. J. Nanomed. 3 (2008) 295-309.

Jójárt-Laczkovich O, Katona G, Aigner Z, Szabó-Révész P. Investigation of recrystallization of amorphous trehalose through hot-humidity stage X-ray powder diffraction. Eur. J. Pharm. Sci. 95 (2016) 145-151.

Junyaprasert V B, Morakul B. Nanocrystals for enhancement of oral bioavailability of poorly watersoluble drugs. Asian J. Pharm. Sci. 10 (2015) 13-23.

Katona G, Sípos P, Frohberg P, Ulrich J, Szabó-Révész P, Jójárt- Laczkovich O. Study of paracetamol-containing pastilles produced by melt technology. J. Therm. Anal. Calorim. 123 (2016) 2549-2559. 
Kayaert P, Anné M, Van den Mooter G. Bead layering as a process to stabilize nanosuspensions: influence of drug hydrophobicity on nanocrystal reagglomeration following in-vitro release from sugar beads. J Pharm Pharmacol. 63 (2011) 1446-1453.

Kevin J. Using Nondestructive in-situ Measurements to Ensure Lyophilized Product Stability. Biopharm Int. 31 (2018) 24-25.

Khadka P, Ro J, Kim H, Kim I, Kim J T, Kim H, Cho J M, Yun G, Lee J. Pharmaceutical particle technologies: An approach to improve drug solubility, dissolution and bioavailability, Asian J. Pharm. Sci. 9 (2014) 304-316.

Kian LK, Jawaid M, Ariffin H, et al. Isolation and characterization of microcrystalline cellulose from roselle fibers. Int. Biol. Macromol. 103 (2017) 931-940.

Kim C J. Surface chemistry and colloids in Advanced pharmaceutics: physico-chemical principles, CRC Press, Florida, United States of America, 2004, 193-256.

Kim H-I, Yeob Park S, Ju Park S, Lee J, Hyung Cho K, Jee J-P, Kim H-C, Maeng H-J, Jang D-J. Development and evaluation of a reconstitutable dry suspension ti improve the dissolution and oral absorption of poorly water-soluble celecoxib. Pharmceutics 10 (2018) $140-154$

Kiss L, Hellinger É, Pilbat AM, Kittel Á, Török Z, Füredi A, Szakács G, Veszelka S, Sipos P, Ózsvári B, Puskás LG, Vastag M, Szabó-Révész P, Deli MA. Sucrose esters increase drug penetration, but do not inhibit p-glycoprotein in caco-2 intestinal epithelial cells. J. Pharm. Sci. 103 (2014) 3107-3119.

Kondo N, Iwao T, Masuda H, Yamanouchi K, Ishihara Y, Yamada N, Haga T, Ogawa Y, Yokoyama K. Improved oral absorption of a poorly watersoluble drug, HO-221, by wet-bead milling producing particles in submicron region. Chem. Pharm. Bull. 41 (1993) 737-740.

Kürti L, Kukovecz Á, Kozma G, Ambrus R, Deli M A, Szabó-Révész P. Study of the parameters influencing the co-grinding process for the production of meloxicam nanoparticles, Powder Technol. 212 (2011) 210-217.

Lee J. Drug nano- and microparticles processed into solid dosage forms:physical properties. $J$. Pharm. Sci. 92 (2003) 2057-2068.

Lee J, Cheng Y. Critical freezing rate in freeze drying nanocrystal dispersions. J. Control. Release 111 (2006) 185-192.

Liu P, Rong X, Laru J,Veen B, Kiesvaara J, Hirvonen J, Laaksonen T, Peltonen L. Nanosuspensions of poorly soluble drugs: Preparation and development by wet milling. Int. J. Pharm. 411 (2011) 215-222.

Liu X, Wang S, Chai L, Zhang D, Suna Y, Xuc L, Sun J. A two-step strategy to design high bioavailable controlled-release nimodipine tablets: The push-pull osmotic pump in 
combination with the micronization/solid dispersion techniques. Int. J. Pharm. 461 (2014) 529-539.

Liversidge G G, Conzentino P. Drug particle size reduction for decreasing gastric irritancy and enhancing absorption of naproxen in rats. Int. J. Pharm. 125 (1995) 309-313.

Liversidge G G, Cundy K C. Particle size reduction for improvement of oral bioavailability of hydrophobic drugs. I. Absolute oral bioavailability of nanocrystalline danazol in beagle dogs. Int. J. Pharm. 125 (1995) 91-97.

Loh Z H, Samanta A K, Heng P W S. Overview of milling techniques for improving the solubility of poorly water-soluble drugs Asian J. Pharm. Sci. 10 (2015) 255-274.

Maggi L, Bruni G, Maietta M, Canobbio A, Cardini A, Conte U. II. Technological approaches to improve the dissolution behavior of nateglinide, a lipophilic insoluble drug: Co-milling. Int. J. Pharm. 454 (2013) 568-572.

Maghsoodi M, Taghizadeh O, Martin GP, Nokhodchi A. Particle design of naproxenmannitol systems containing crystalline microcomposites. J. Pharm. Biomed. Anal. 56 (2011) 183-190.

Malamatari M, Somavarapu S, Taylor KMG et al. Solidufication of nanosuspensions for the production of solid oral dosage forms and inhalable dry powders. Exp. Opin. Drug. Del. 13 (2016) 435-450.

Malamatari M, Taylor K M G, Malamataris S, Douroumis D, Kachrimanis K. Pharmaceutical nanocrystals: production by wet milling and applications. Drug Discov. Today. 23 (2018) 534-547.

McNeill I C, Ahmed S, Memetea L T. Thermal degradation of vinyl acetate-methacrylic acid copolymer and the homopolymers. II. Thermal analysis studies. Polym. Degrad. Stabil. 48 (1995) 89-97.

Merisko-Liversidge E, Liversidge G G. Nanosizing for oral and parenteral drug delivery: A perspective on formulating poorly-water soluble compounds using wet media milling technology. Adv. Drug Deliver. Rev. 63 (2011) 427-440.

Merisko-Liversidge E, Sarpotdar P, Bruno J, Hajj S, Wei L, Peltier N, Rake J, Shaw J M, Pugh S, Polin L, Jones J, Corbett T, Cooper E, Liversidge G G. Formulation and antitumor activity evaluation of nanocrystalline suspensions of poorly soluble anticancer drugs. Pharm. Res. 13 (1996) 272-278.

Mittal B. How to Develop Robust Solid Oral Dosage Forms from Conception to PostApproval, Chapter 4 - Pharmaceutical Unit Operations. Academic Press (2017) 69-95.

Moorthi C, Kathiresan K. Fabrication of highly stable sonication assisted curcumin nanocrystals by nanoprecipitation method. Drug Inv. Today 5 (2013) 66-69. 
Mouton J W, van Peer A, de Beule K, Van Vliet A, Donnelly J P, Soons P A. Pharmacokinetics of itraconazole and hydroxyitraconazole in healthy subjects after single and multiple doses of a novel formulation. Antimicrob. Agents Chemother. 50 (2006) 4096-4102.

Möschwitzer J, Müller RH. Spray coated pellets as carrier system for mucoadhesive drug nanocrystals. Eur J Pharm Biopharm. 62 (2006) 282-287.

Müller R, Junghanns J. Drug Nanocrystals/Nanosuspensions for the Delivery of Poorly Soluble Drugs, Nanoparticulates as Drug Carriers (1. 1.), Imperial College Press, London, 2006. 307-328.

Na G C, Stevens H J, Yuan B O, Rajagopalan N. Physical Stability of Ethyl Diatrizoate Nanocrystalline Suspension in Steam Sterilization. Pharm. Res. 16 (1999) 569-574.

Nagy Z K, Balogh A, Drávavölgyi G, Ferguson J, Pataki H. Solvent-Free Melt Electrospinning for Preparation of Fast Dissolving Drug Delivery System and Comparison with Solvent-Based Electrospun and Melt Extruded Systems. Wiley Online Library (2012) DOI 10.1002/jps.23374

Naik S, Chaudhuri B. Quantifying Dry Milling in Pharmaceutical Processing: A Review on Experimental and Modeling Approaches. J. Pharm. Sci. 104 (2015) 2401-2413.

Nalluri V R, Kuentz M. Advancing Pharmaceutical Dry Milling by Process Analytics and Robustness Testing. J. Pharm. Innov. 5 (2010) 100-108.

Oberle R L, Moore T J, Krummel D A P. Evaluation of mucosal damage of surfactants in rat jejunum and colon. J. Pharmacol. Tox. Met. 33 (1995) 75-81.

Ochi M, Kawachi T, Toita E, Hashimoto I, Yuminoki K, Onoue S, Hashimoto N. Development of nanocrystal formulation of meloxicam with improved dissolution and pharmacokinetic behaviors. Int. J. Pharm. 474 (2014) 151-156.

Ostrander K D, Bosch H W, Bondanza D M. An in-vitro assessment of a NanoCrystalTM beclomethasone dipropionate colloidal dispersion via ultrasonic nebulization. Eur. J. Pharm. Biopharm. 48 (1999) 207-215.

Peltonen, L, Hirvonen, J. Pharmaceutical nanocrystals by nanomilling: critical process parameters, particle fracturing and stabilization methods. J. Pharm. Pharmacol. 62 (2010) 1569-1579.

Perry J H. Size reduction, Chemlcnl Engmeenng Handbook, McGraw-Hill, New York, 4th edition (1963) 8-8.

Pomázi A, Ambrus R, Sipos P, Szabó-Révész P. Analysis of co-spray-dried meloxicammannitol systems containing crystalline microcomposites. J. Pharmaceut. Biomed. 56 (2011) 183-190. 
Pomázi A, Buttini F, Ambrus R, Colombov P, Szabó-Révész P. Effect of polymers for aerolization properties of mannitol-based microcomposites containing meloxicam. Eur. Polym. J. 49 (2013) 2518-2527.

Pourghahramani P, Forssberg E. Effects of mechanical activation on the reduction behavior of hematite concentrate. Int. J. Miner. Process. 82 (2007) 96-105.

Prasad K L, Smyth H. 3D Printing technologies for drug delivery: a review. Drug Dev Ind Pharm. 42 (2016) 1019-1031.

Rafiq S I, Jan K, Singh S, Saxena D C. Physicochemical, pasting, rheological, thermal and morphological properties of horse chestnut starch. J. Food Sci \& Tech. 52 (2015) 5651-5660

Shaimaa A, Thirumala G, Inamullah K, ur Rehman N, Ali W, Shah S M H, Khan S, Hussain Z, Ullah R, S Alsaid M. Experimental and molecular modeling approach to optimize suitable polymers for fabrication of stable fluticasone nanoparticles with enhanced dissolution and antimicrobal activity. Drug. Des. Dev. Ther. 12 (2018) 255-269.

Shegokar R, Müller R H. Nanocrystals: Industrially feasible multifunctional formulation technology for poorly soluble actives, Int. J. of Pharm. 399 (2010) 129-139.

Siewert C, Moog R, Alex R, Kretzer P, Rothenhäusler B. Process and scaling parameters for wet media milling in early phase drug development: A knowledge-based approach. Eur. J. Pharm. Sci. 115 (2018) 126-131.

Simmons DL, Botting RM, Hla T. Cyclooxygenase isozymes: the biology of prostaglandin synthesis and inhibition. Pharmacol Rev. 56 (2004) 387-437.

Suryanarayana C. Mechanical alloying and milling. Prog. Mater. Sci. 46 (2001) 1-184.

Sussich F, Cesàro A. Trehalose amorphization and recrystallization. Carbohyd. Re. 343 (2008) 2667-2674.

Szabó-Révész P. Modifying the physicochemical properties of NSAIDs for nasal and pulmonary administration. Drug Discov. Today: Technol. 27 (2018) 87-93.

Tretinnikov O N, Zagorskaya S A. Determination of the degree of crystallinity of poly(vinyl alcohol) by FTIR spectroscopy. J. App. Spectrosc. 79 (2012) 521-526.

Ujhelyi Z, Fenyvesi F, Váradi J, Fehér P, Kiss T, Veszelka Sz, Deli M, Vecsernyés M, Bácskay I. Evaluation of cytotoxicity of surfactants used in self-micro emulsifying drug delivery systems and their effects on paracellular transport in Caco-2 cell monolayer. Eur. J. Pharm. Sci. (2012) 564-573.

Van Eerdenbrugh B, Froyen L, Van Humbeeck J, A. Martens J, Augustijns P, Van Den Mooter G. Alternative matrix formers for nanosuspension solidification: Dissolution performance and X-ray microanalysis as an evaluation tool for powder dispersion. Eur. J. Pharm. Sci. 35 (2008) 344-353. 
Van Eerdenbrugh B, Froyen L, Jan Van Humbeeck, Johan A. Martens, Patrick Augustijns, Guy Van Den Mooter, Drying of crystalline drug nanosuspensions-The importance of surface hydrophobicity on dissolutio behavior upon redispersion. Eur. J. Pharm. Sci. 35 (2008) 127-135.

Van Eerdenbrugh B, Van den Mooter G, Augustijns P. Top-down production of drug nanocrystals: nanosuspension stabilization, miniaturization and transformation into solid products. Int. J. Pharm. 364 (2008) 64-75.

Ventosa N, Sala S, Veciana J, Torres J, Llibre J. Depressurization of an Expanded Liquid Organic Solution (DELOS): a new procedure for obtaining submicron- or micron-sized crystalline particles. Cryst. Growth Des. 1 (2001) 299-303.

Vergote G J, Nervaet C, Van Driessche I, Hoste S, De Smedt S, Demeester J, Jain R A, Ruddy S, Remon J P . An oral controlled release matrix pellet formulation containing nanocrystalline ketoprofen, Int. J. Pharm. 219 (2001) 81-87.

Verma S, Kumar S, Gokhale R, J.Burgess D. Physical stability of nanosuspensions: Investigation of the role of stabilizers on Ostwald ripening. Int. J. Pharm. 406 (2011) 145152.

Wahlstrom J L, Chiang P C, Ghosh S, Warren C J, Wene S P, Albin L A, Smith M E, Roberds, S L. Pharmacokinetic evaluation of a 1,3-dicyclohexylurea nanosuspension formulation to support early efficacy assessment. Nanoscale Res. Lett. 2 (2007) 291-296.

Wang Y, Zheng Y, Zhang L, Wang Q, Zhang D. Stability of nanosuspensions in drug delivery. J. Control. Release 172 (2013) 1126-1141.

Web reference 6 Retsch ${ }^{\circledR}:$ http://pdf.directindustry.com/pdf/retsch/the-sample-high-energyballmills/19308-518973.html

Wiedmann T S, DeCastro L, Wood R W. Nebulization of NanoCrystalsTM: production of a respirable solid-in-liquid-in-air colloidal dispersion. Pharm. Res. 14 (1997) 112-116.

Wu L, Zhang J, Watanabe W. Physical and chemical stability of drug nanoparticles. Adv. Drug Deliver. Rev. 63 (2011) 456-469.

Yim J-H, Kim W-S, Lim J S. Recrystallization of Adefovir Dipivoxil Particles Using the Aerosol Solvent Extraction System Process. Ind. Eng. Chem. Res. 53 (2014) 1663-1671.

Zhang Y, Fei S, Yu M, Guo Y, He H, Zhang Y, Yin T, Xu H, Tang X. Injectable sustained release PLA microparticles prepared by solvent evaporation-media milling technology. Drug Dev. Ind. Pharm. 44 (2018), 1591-1597.

Zheng J Y, Bosch, H W. Sterile filtration of NanoCrystalTM drug formulations. Drug Dev. Ind. Pharm. 23 (1997) 1087-1093. 


\section{ACKNOWLEDGEMENTS}

I express my grateful thanks to Dr. Ildikó Csóka, head of the Ph.D. programme Pharmaceutical Technology, for providing me with the possibility to complete my work under his guidance.

I am grateful to my supervisors Prof. Dr. Piroska Szabó-Révész for her scientific guidance, encouragement and support throughout my PhD studies.

I am thankful to Dr. Mária Budai-Szücs, Dr. Csilla Bartos and Dr. Gábor Katona for their inspiring help in my studies.

I am grateful to co-authors for cooperation and their great help.

I would like to thank Erika Boda and Zoltánné Lakatos for excellent technical assistance.

Finally, I am especially thankful to my family for their love and untiting support during my studies.

This work was supported by Gedeon Richter's Talentum Foundation, Richter Plc., Budapest, Hungary.

This work was supported by the National Research Development and Innovation Office and Richter Plc., Budapest, Hungary (GINOP 2.2.1 15-2016-00007)

This work was supported by Ministry of Human Capacities, Hungary grant 20391 3/2018/FEKUSTRAT. 
3. sz. melléklet: nyilatkozat az értekezés eredetiségéről

\section{NYILATKOZAT SAJÁT MUNKÁRÓL}

Név: Bartos Csaba

A doktori értekezés címe: Optimization of a combined wet milling process to produce nanosuspension and its transformation into surfactant-free solid compositions to increase the product stability and drug bioavailability

Én, Bartos Csaba teljes felelősségem tudatában kijelentem, hogy a Szegedi Tudományegyetem Gyógyszertudományok Doktori Iskolában elkészített doktori (Ph.D.) disszertációm saját kutatási eredményeimre alapulnak. Kutatómunkám, eredményeim publikálása, valamint disszertációm megírása során a Magyar Tudományos Akadémia Tudományetikai Kódexében lefektetett alapelvek és ajánlások szerint jártam el.

Szeged, 2019. november 20. 
ANNEX 


\section{PUBLICATION I.}


Article

\title{
The Effect of an Optimized Wet Milling Technology on the Crystallinity, Morphology and Dissolution Properties of Micro- and Nanonized Meloxicam
}

\author{
Csilla Bartos ${ }^{1}$, Piroska Szabó-Révész ${ }^{1}$, Csaba Bartos ${ }^{1}$, Gábor Katona ${ }^{1,2}$, \\ Orsolya Jójárt-Laczkovich ${ }^{1}$ and Rita Ambrus ${ }^{1, *}$ \\ 1 Department of Pharmaceutical Technology, University of Szeged, Eötvös u. 6, H-6720 Szeged, Hungary; \\ bartoscsilla@pharm.u-szeged.hu (C.B.); revesz@pharm.u-szeged.hu (P.S.-R.); \\ bartoscsaba@pharm.u-szeged.hu (C.B.); katona@pharm.u-szeged.hu (G.K.); \\ laczkovo@pharm.u-szeged.hu (O.J.-L.) \\ 2 Gedeon Richter Plc., Gyömrői út 19-21, H-1103 Budapest, Hungary \\ * Correspondence: arita@pharm.u-szeged.hu; Tel.: +36-6254-5575; Fax: +36-6254-5571 \\ Academic Editor: Sohrab Rohani \\ Received: 22 March 2016; Accepted: 13 April 2016; Published: 21 April 2016
}

\begin{abstract}
This article reports on the effects of a new combined wet milling technique on the physicochemical properties of meloxicam (MEL). The influence of milling time on the particle size, the crystallinity, the morphology and the dissolution rate of MEL has been studied in the presence and absence of polyvinyl alcohol (PVA) as a stabilizer agent. Micronized MEL particles were produced in aqueous medium which did not contain additive after milling for $10 \mathrm{~min}$. For nanonization an additive and longer milling time were required. After particle size determination the structural and morphological characterization of the wet milled, dried products containing MEL were studied. X-ray powder diffractometry (XRPD) and differential scanning calorimetry (DSC) examinations revealed the change in the crystallinity of MEL. Scanning electron microscopy (SEM) images showed that aggregates of nanosized MEL particles were formed, regardless of the presence of PVA. The nanonized MEL crystals $\left(D_{50}=126 \mathrm{~nm}\right)$ exhibited a regular shape and a smooth surface. The increased specific surface area resulted in a high dissolution rate and concentration of free MEL. According to the results, the produced samples could be applied as a basic material (micronized MEL) and intermediate product (micronized and nanonized MEL with PVA) for the design of dosage forms.
\end{abstract}

Keywords: wet milling; micronization; nanonization; physical structure; crystallinity; in vitro dissolution

\section{Introduction}

One of the major current challenges of the pharmaceutical industry is related to strategies that improve the water solubility of drugs because over $40 \%$ of new drug candidates are water-insoluble compounds [1]. Poorly water-soluble drug properties can impede the effective delivery of these drugs into humans, and affect their dissolution rate and subsequent absorption at the site of activity [2].

Poorly soluble molecules have been successfully formulated by employing a variety of techniques to modify the physico-chemical and biopharmaceutical properties of drugs $[3,4]$ such as: (i) solubilization in surfactant solutions; (ii) use of co-solvents [5], salt formation [6], complexation with cyclodextrins [7], crystallization [8], amorphization [9], milling [10], etc.

Milling is a technique commonly applied to produce micro- or nanosized drug crystals in order to increase the dissolution rate and absorption, and hence the bioavailability of poorly-soluble materials. According to the Noyes-Whitney equation, the reduction of the particle sizes of drug crystals increases the specific surface area, which can improve the rate of dissolution of the drug [11]. There are many different well-known types of milling techniques with both advantages and disadvantages; dry and 
wet milling can be distinguished [12,13]. Wet milling requires less energy and time than dry milling. Thanks to the environmentally isolated system, it is a dust free process and the material is less heated up [14]. However, wet milling has some disadvantages as well, e.g., increased wear of the grinding medium, corrosion hazards, etc. To overcome the limitations of the conventional particle size reduction technologies for poorly-soluble drugs, new combinational methods have been developed for the production of ultra-fine suspensions. These technologies are a relatively new approach to improve the effectiveness of particle size reduction and to reduce the time of processes. In general, they can be described as a combination of a bottom-up process (the building-up of particles) [15-17] followed by a top-down technology (disintegration) $[18,19]$. This method involves two particle size reduction steps. There is also a possibility for the combination of dry- and wet milling in one step, but literature data relating to the application of this combined method are lacking. Retsch GmbH (Haan, Germany) has recommended the combination of planetary ball milling, as dry milling, and pearl milling, as wet milling [20] techniques.

In wet micro- and nanonization, the application of additives is required in order to retain the individuality of the particles. Different additives are used to stabilize these particles: poly(vinylpyrrolidone) (PVP), Poloxamer ${ }^{\circledR}$ (Poloxamer 188 = poly(ethylene)-poly(propylene glycol), polysorbate $\left(\right.$ Tween $80^{\circledR}=$ poly $\left(\right.$ oxyethylenesorbitan monooleate)), Solutol ${ }^{\circledR}$ (Solutol HS $15=$ poly(ethylene glycol 15-hydroxystearate)), PVA (poly(vinyl alcohol)), etc. [21].

Milling and the presence of additives during milling have the ability to decrease the crystallinity of active materials [22,23]. The amorphous phase transition usually results in improved dissolution rates, thereby increasing the bioavailability, with the improvement being directly related to the extent of amorphization [24]. However, physical and chemical instability of amorphous material could be expected.

Meloxicam (MEL) is a non-steroidal anti-inflammatory drug (NSAID) with anti-inflammatory, analgesic and antipyretic effects. MEL was chosen as a model crystalline drug because of its poor aqueous solubility $(4.4 \mu \mathrm{g} / \mathrm{mL})[25]$ and relatively high melting point $\left(270{ }^{\circ} \mathrm{C}\right)$ [26].

This research investigates the applicability of a wet milling method combining planetary ball and pearl milling. We set out to utilize particle size reduction to the micro- or nanometre range. The effects of milling time and the presence or absence of stabilizer (PVA) in reducing the particle size were investigated. The changes in crystallinity and morphology of MEL during milling have been studied. The effects of particle size reduction and amorphization on the dissolution rate were determined.

\section{Results and Discussion}

\subsection{Effects of Milling Time on Particle size Distribution (PSD) and Specific Surface Area (SSA)}

During our work, a combined wet milling technique (planetary ball mill and pearls, as milling media) was investigated. Analysis of the laser diffraction results revealed that the milling in the case of samples containing water as a dispersant medium resulted in a roughly $85 \%$ decrease in average MEL particle size. The $\mathrm{D}_{10}, \mathrm{D}_{50}$ and $\mathrm{D}_{90}$ values and the SSA are reported in Table 1.

After 10 min milling, micronized MEL was observed. However, further milling led to an increase in particle size, because MEL particles aggregated without the presence of PVA. The smallest average particle size $(3.55 \mu \mathrm{m})$ and the highest SSA $\left(1.89 \mathrm{~m}^{2} / \mathrm{g}\right)$ were obtained during the first $10 \mathrm{~min}$ of milling. During milling heat production was observed and the temperature of the samples increased up to $40-45^{\circ} \mathrm{C}$. The aqueous solubility of MEL $\left(4.4 \mu \mathrm{g} / \mathrm{mL}, 25^{\circ} \mathrm{C}\right)$ increased due to the elevated temperature $\left(40 \mu \mathrm{g} / \mathrm{mL}, 37^{\circ} \mathrm{C}\right.$ ), but it was not remarkable in our investigation (at about $0.04 w / v \%$ ), and $0.8 \mathrm{mg}$ MEL could be dissolved in our dispersant medium (water) of the presence $2 \mathrm{~g}$ of active agent. The recrystallization of the small amount of dissolved MEL was irrelevant in terms of any particle size increase. 
Table 1. MEL PSD and SSA in pre-dispersions containing water as dispersant.

\begin{tabular}{|c|c|c|c|c|}
\hline Milling Time (min) & $\mathrm{D}_{10}(\mu \mathrm{m})$ & $D_{50}(\mu \mathrm{m})$ & $\mathrm{D}_{90}(\mu \mathrm{m})$ & $\mathrm{SSA}\left(\mathrm{m}^{2} / \mathrm{g}\right)$ \\
\hline raw MEL & 11.40 & 34.26 & 73.59 & 0.332 \\
\hline 10 & 1.76 & 3.55 & 8.69 & 1.89 \\
\hline 20 & 2.63 & 5.74 & 14.21 & 1.22 \\
\hline 30 & 2.62 & 5.99 & 18.97 & 1.18 \\
\hline 40 & 2.77 & 6.84 & 24.54 & 1.07 \\
\hline 50 & 2.72 & 6.13 & 19.56 & 1.15 \\
\hline 60 & 2.57 & 6.12 & 20.87 & 1.18 \\
\hline 70 & 2.77 & 7.13 & 26.26 & 1.05 \\
\hline 80 & 2.58 & 5.94 & 22.54 & 1.19 \\
\hline 90 & 2.51 & 5.55 & 18.88 & 1.25 \\
\hline
\end{tabular}

In the case of PVA-solution as a dispersant, after 10 min micronized, and after 50 min nanonized particles were noted, with monodisperse PSD (Table 2). The average particle size decreased efficiently, from approximately $35 \mu \mathrm{m}$ to $126 \mathrm{~nm}$, up to $50 \mathrm{~min}$ of milling. The SSA of MEL rose 150-fold compared to the raw MEL. Further milling led to only a slight decrease in particle size. PVA, used as a coating polymer helped the particles to separate from each other. Aggregation was also prevented, therefore the stability of the system could be improved.

Table 2. MEL PSD and SSA in pre-dispersions containing PVA-solution as dispersant.

\begin{tabular}{ccccc}
\hline Milling Time $(\mathbf{m i n})$ & $\mathbf{D}_{\mathbf{1 0}}(\boldsymbol{\mu} \mathbf{m})$ & $\mathbf{D}_{\mathbf{5 0}}(\mu \mathbf{m})$ & $\mathbf{D}_{\mathbf{9 0}}(\mu \mathbf{m})$ & $\left.\mathbf{S S A ~}_{\mathbf{( m}} \mathbf{~} \mathbf{g}\right)$ \\
\hline raw MEL & 11.40 & 34.26 & 73.59 & 0.332 \\
10 & 0.24 & 2.96 & 29.40 & 7.71 \\
20 & 0.084 & 0.169 & 2.863 & 34.6 \\
30 & 0.090 & 0.337 & 4.180 & 27.2 \\
40 & 0.074 & 0.134 & 1.358 & 46 \\
50 & 0.074 & 0.126 & 0.253 & 49.5 \\
60 & 0.072 & 0.122 & 0.219 & 52 \\
70 & 0.070 & 0.121 & 0.225 & 52.6 \\
80 & 0.072 & 0.119 & 0.209 & 53.2 \\
90 & 0.064 & 0.116 & 0.219 & 57.9 \\
\hline
\end{tabular}

\subsection{Characterization of the Dried Products}

\subsubsection{Physical Structure (XRPD and DSC)}

For determination the effect of milling on the crystallinity of MEL pre-dispersions, samples milled for $10 \mathrm{~min}$ in water as a dispersant, and for 10 and 50 in PVA-solution as a dispersant were dried and characterized.

The XRPD diffractogram of raw MEL and of MEL in the physical mixture of MEL-PVA demonstrated its crystalline structure, as expected. The characteristic peaks are located at the following $2 \theta$ values: $13.22^{\circ}, 15.06^{\circ}$ and $26.46^{\circ}$. Ten min of milling in the water-containing sample caused the loss of the crystalline structure of the drug, and only $3 \%$ of the drug remained crystalline (Table 3 ). The intensities of the characteristic peaks were decreased in the case of the treated product (Figure 1). During the further milling aggregation was observed, because of the lack of PVA. At the end of milling (90 $\mathrm{min}$ ), $9 \%$ of MEL remained crystalline.

In the PVA-solution containing sample in the course of milling, a decrease in crystallinity was perceptible. After milling for $10 \mathrm{~min}, \sim 47 \%$ of the drug remained crystalline. After 90 min milling amorphization was detected, the degree of crystallinity was $2 \%$ (Table 3 ). The intensities of the characteristic peaks decreased due to the milling (Figure 2). 


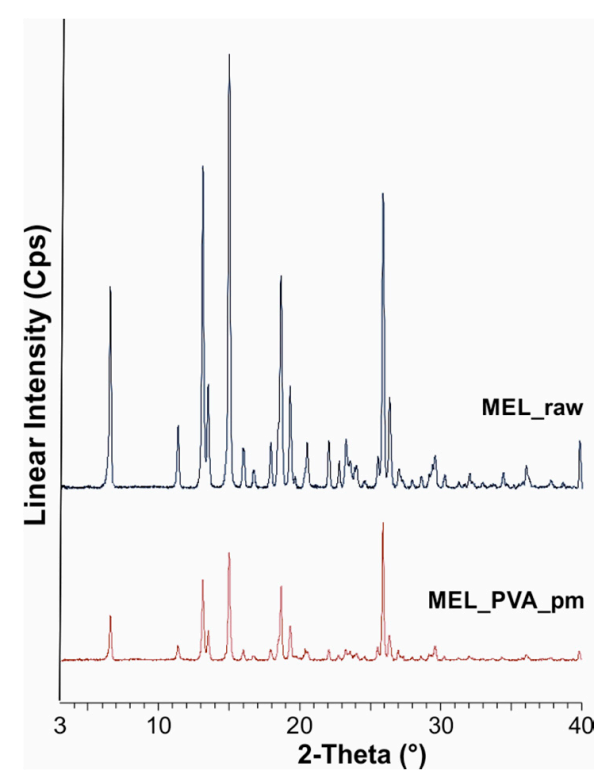

Figure 1. XRPD examination of MEL_raw and MEL_PVA_pm.

Table 3. The degree of MEL crystallinity (\%) for different dispersant media and milling times.

\begin{tabular}{cc}
\hline Sample & Crystallinity (\%) \\
\hline MEL_raw & 100 \\
MEL_PVA_pm & 80 \\
MEL_W 10 min & 3 \\
MEL_W 50 min & 5 \\
MEL_W 60 min & 8 \\
MEL_W 90 min & 9 \\
MEL_W_PVA 10 min & 48 \\
MEL_W_PVA 50 min & 12 \\
MEL_W_PVA 60 min & 5 \\
MEL_W_PVA 90 min & 2 \\
\hline
\end{tabular}

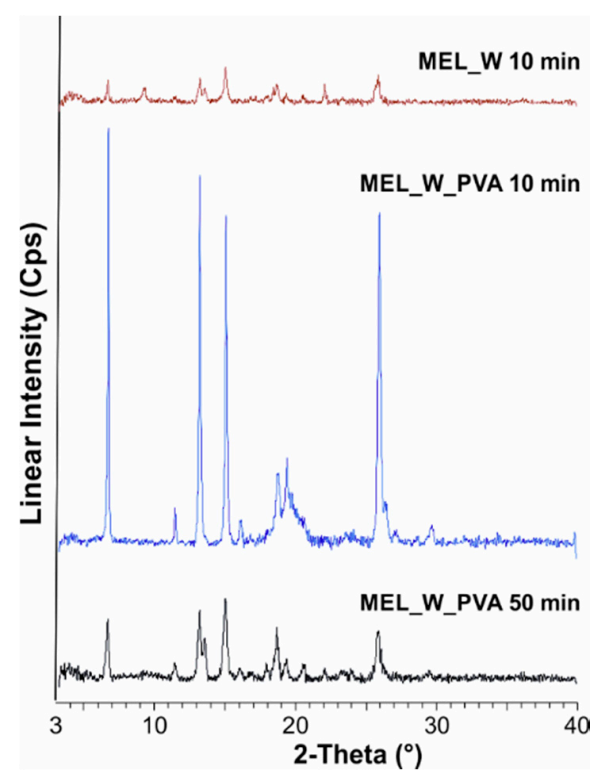

Figure 2. XRPD examination of MEL_W $10 \mathrm{~min}, \mathrm{MEL} \_W \_P V A 10 \mathrm{~min}$ and MEL_W_PVA 50 min. 
DSC was employed to investigate the melting of MEL in the raw form, the physical mixture and in the milled dried products (Figure 3). The DSC curves of the raw MEL and of MEL in the physical mixture of MEL-PVA revealed a sharp endothermic peak at 259.1 and $255.9{ }^{\circ} \mathrm{C}$, reflecting its melting point and confirming its crystalline structure. In case of water-containing samples the most significant decrease of crystallinity was observed after $10 \mathrm{~min}$ milling (peak: $227.0^{\circ} \mathrm{C}$ ). Increasing the milling time, aggregation was detected. Using PVA-solution as dispersant, DSC curves exhibited a broad endothermic peak for MEL, indicating that the crystallinity of the drug was decreased (Table 4). The residual MEL crystals in the products melted at a lower temperature than the crystals of raw MEL due to the smaller particle size and the increased degree of amorphization which was directly proportional to the duration of milling. This process was promoted by PVA, which was softened at $43^{\circ} \mathrm{C}$ as glass transition temperature $\left(T_{g}\right)$ value.

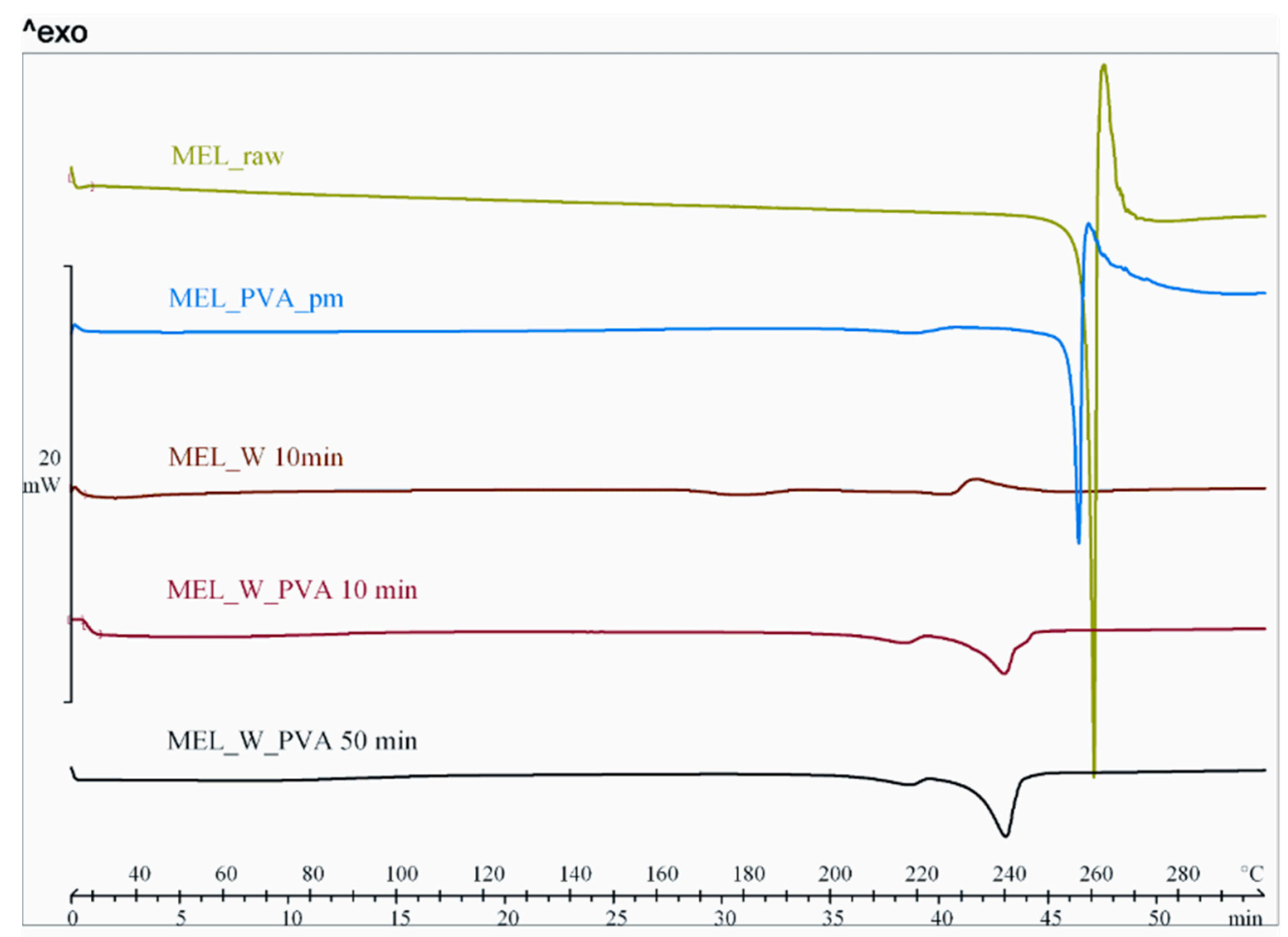

Figure 3. DSC curves of MEL_raw, MEL_PVA_pm, MEL_W $10 \mathrm{~min}$, MEL_W_PVA $10 \mathrm{~min}$ and MEL_W_PVA $50 \mathrm{~min}$.

Table 4. Thermoanalytical evaluation of MEL_raw, MEL_PVA_pm and dried milled products.

\begin{tabular}{ccccccccccc}
\hline Parameters & Raw MEL & pm & \multicolumn{3}{c}{ Dried MEL_W Samples } & \multicolumn{3}{c}{ Dried MEL_W_PVA Samples } \\
\hline Milling time (min) & - & - & $\mathbf{1 0}$ & $\mathbf{5 0}$ & $\mathbf{6 0}$ & $\mathbf{9 0}$ & $\mathbf{1 0}$ & $\mathbf{5 0}$ & $\mathbf{6 0}$ & $\mathbf{9 0}$ \\
$\boldsymbol{\Delta} \mathbf{H}^{*}(\mathbf{J} / \mathbf{g})$ & -148.5 & -29.9 & -7.5 & -40.1 & -64.4 & -40.8 & -60.3 & -70.9 & -32.4 & -46.4 \\
Onset $\left({ }^{\circ} \mathbf{C}\right)$ & 258.4 & 235.9 & 217.9 & 233.5 & 248.5 & 247.1 & 231.6 & 232.9 & 207.1 & 206.2 \\
Peak $\left({ }^{\circ} \mathbf{C}\right)$ & 259.1 & 255.9 & 227.0 & 238.7 & 250.7 & 250.2 & 239.8 & 240.1 & 216.7 & 217.4 \\
Endset $\left({ }^{\circ} \mathbf{C}\right)$ & 261.0 & 255.9 & 230.0 & 240.4 & 250.8 & 251.6 & 243.1 & 243.2 & 222.3 & 222.3 \\
\hline
\end{tabular}

It can be concluded that combined milling resulted in partial amorphization of MEL which could results in a higher dissolution rate compared to the raw drug and its physical mixtures.

\subsubsection{Particle Shape and Size}

In the following SEM images (Figure 4) the morphology of the modified particles is demonstrated. Dried products produced by milled pre-dispersion in water for $10 \mathrm{~min}$ and MEL micronized and nanonized in the presence of PVA (10 and $50 \mathrm{~min}$ ) were investigated. How the surface and shape 
changed after the milling process, compared with raw MEL, was checked. After taking samples after $10 \mathrm{~min}$ of milling in the case of water as a dispersant and 10 and $50 \mathrm{~min}$ of milling in the case of PVA-solution as the medium, the suspensions were dried and characterized. The raw MEL consisted mainly of angular, prismatic crystals with a broad size distribution. In the case of the water-containing samples, it was shown that micro-sized aggregates consisting of nanonized particles were formed. In the case of PVA-solution-containing sample, $10 \mathrm{~min}$ of milling resulted in irregular particle shapes, with the approximately $3 \mu \mathrm{m} \mathrm{D}_{50}$ value. MEL crystals could be detected between the amorphous PVA particles. After $50 \mathrm{~min}$, nanonized MEL crystals (at about $120 \mathrm{~nm}$ ) embedded in the PVA-film, with regular shape and smooth surface were perceived. The particle endurance during the treatment accounts for the smooth surfaces of the particles.
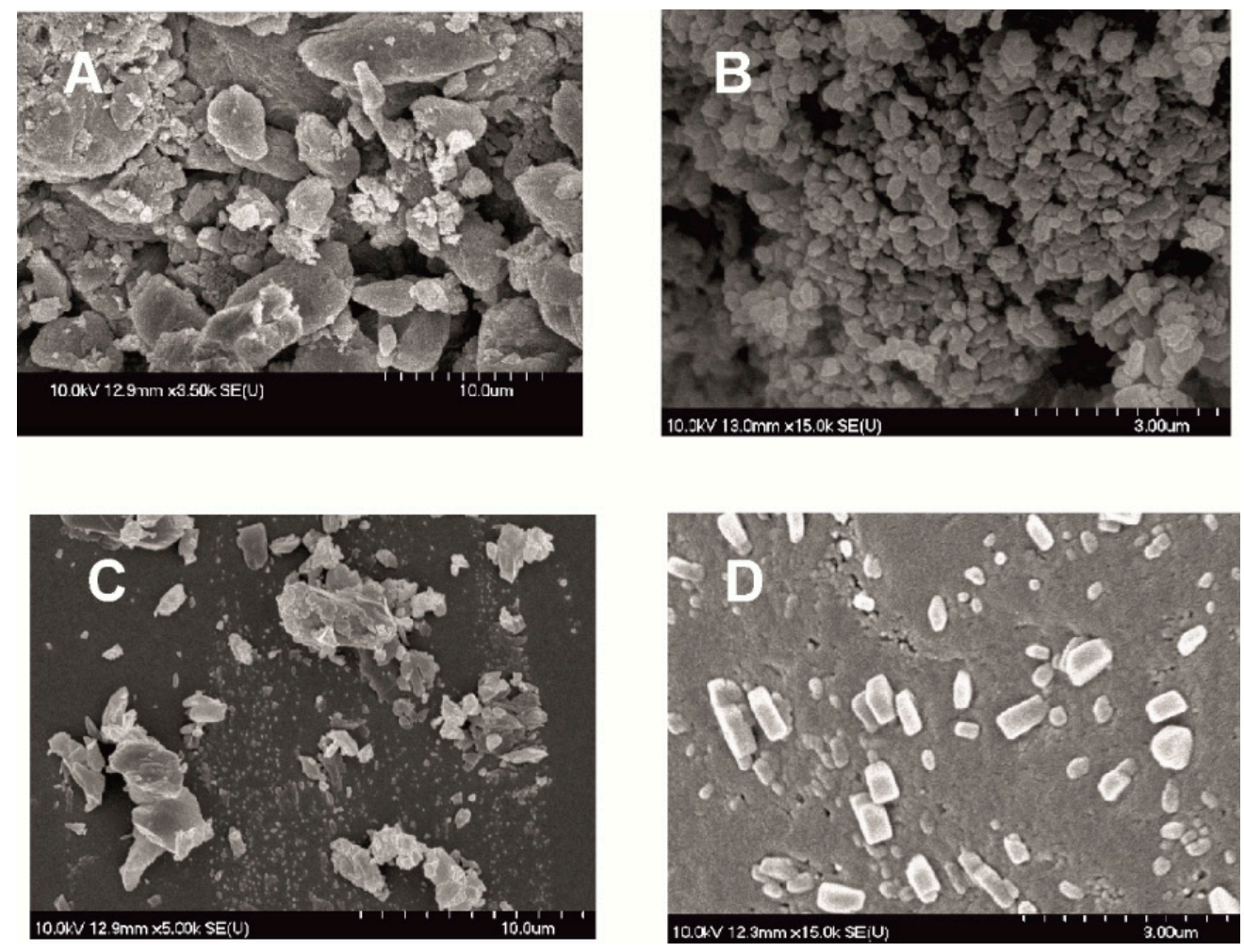

Figure 4. SEM pictures of MEL_raw (A); MEL_W $10 \mathrm{~min}$ (B); MEL_W_PVA $10 \mathrm{~min}$ (C) and MEL_W_PVA 50 min (D).

\subsubsection{In Vitro Dissolution Tests}

The in vitro dissolution test was performed at $\mathrm{pH} 7.4$, which could represent the media of the intestinal system where the absorption of MEL could be totally achieved. At pH 7.4 the conditions of the lung, nasal and skin epithelia could also be imitated. The in vitro dissolution showed the poor solubility and slow dissolution of MEL $\left(\mathrm{D}_{50}=34.26 \mu \mathrm{m}\right)$, and after $60 \mathrm{~min}$ approximately $10 \%$, and after $2 \mathrm{~h}$ around $22 \%$ of the drug was liberated. Application of PVA (in the reference sample, where the size of the MEL did not decreased significantly) as hydrophilic wetting agent, could improve the dissolution rate by 2 -fold. The micronization of the drug without PVA $\left(\mathrm{D}_{50}=3.55 \mu \mathrm{m}\right)$ could influence the dissolution rate 3-fold. Producing individual micronized $\left(D_{50}=2.96 \mu \mathrm{m}\right)$ and nanonized $\left(\mathrm{D}_{50}=0.126 \mu \mathrm{m}\right)$ MEL drug particles in the presence of PVA, the extent of dissolution was enhanced significantly by the increased specific surface area. The amorphous character and the submicron range of the drug resulted in a very fast dissolution, with more than $70 \%$ of MEL liberated after the first 5 min (Figure 5). 


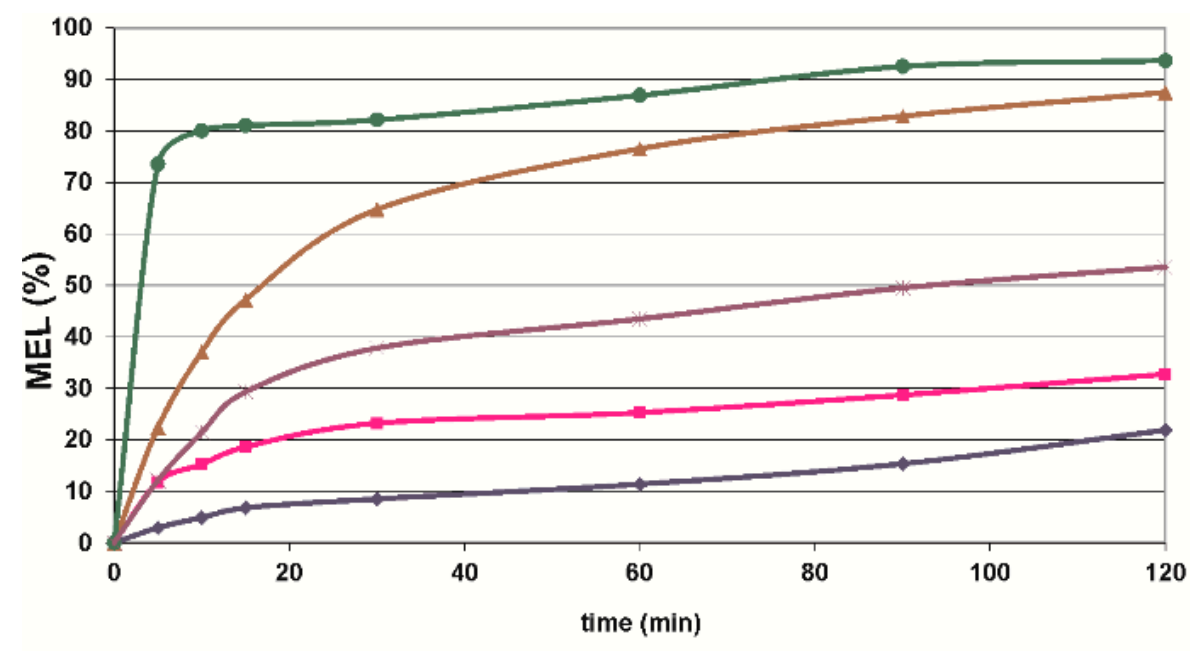

$\rightarrow$ MEL_raw $\rightarrow$ MEL_PVA_pm $\rightarrow$ MEL_W_PVA 10min $\rightarrow$ MEL_W_PVA 50min $\rightarrow$ MEL_W 10min

Figure 5. In vitro dissolution profile of MEL_raw, MEL_PVA_pm, MEL_W 10 min, MEL_W_PVA $10 \mathrm{~min}$ and MEL_W_PVA $50 \mathrm{~min}(\mathrm{SD}<0.05 \%)$.

\section{Experimental Section}

\subsection{Materials}

Meloxicam (4-hydroxy-2-methyl- $\mathrm{N}$-(5-methyl-2-thiazolyl)-2H-benzothiazine-3-carboxamide-1,1dioxide) was obtained from EGIS Ltd. (Budapest, Hungary). The grinding additive, PVA (polyvinyl alcohol), was purchased from Gedeon Richter Plc. (Budapest, Hungary).

\subsection{Methods}

\subsubsection{Combined Milling}

A wet milling technique (a combination of planetary ball and pearl milling) was employed. The drug ( $2 \mathrm{~g}$ ) was first suspended in the dispersant medium $(18 \mathrm{~g})$, consisting of water and aqueous solution of PVA (the use of lower concentrations of PVA led to extensive aggregation of the MEL particles because of the lesser effectiveness in overcoming the cohesive forces). The resulting suspensions (10\% drug content) were wet milled at $400 \mathrm{rpm}$ in the milling chamber $(50 \mathrm{~mL})$ of a planetary ball mill (PM $100 \mathrm{MA}$, Retsch $\mathrm{GmbH}$, Haan, Germany). The milling balls were $0.3 \mathrm{~mm} \mathrm{ZrO}_{2}$ beads. As a preliminary experiment, the effects of different pearl weights $(10,20,50$ and $150 \mathrm{~g})$ on the particle size reduction were investigated, and milling was also carried out without pearls as a benchmark. The most effective milling was observed when $20 \mathrm{~g}$ of pearls was applied. Increasing the weight of pearls did not result in further particle size reduction, therefore $20 \mathrm{~g}$ of beads were used for further experiments. The milled suspensions were separated with a $150 \mu \mathrm{m}$ mesh size sieve. Suspension sampling was carried out at milling times of 10, 20, 30, 40, 50, 60, 70, 80 and 90 (end of milling) min to perform the particle size analysis.

\subsubsection{Determination of Particle Size Distribution and Specific Surface Area by a Laser Diffraction Method}

The volume based particle size distribution (PSD) of the raw MEL was measured by laser diffraction (Mastersizer 2000, Malvern Instruments Ltd., Worcestershire, UK) with the following parameters: 300RF lens; small volume dispersion unit (2500 rpm); refractive index for dispersed particles 1.720; refractive index for dispersion medium 1.330. The Dynamic Laser Light Scattering method was used to determine the PSD. The particle size of the MEL was determined directly on the initial suspension in water in which PVA was dissolved. The size analysis was repeated three times. Water was used as dispersant medium and the obscuration was in the range $11 \%-16 \%$ for 
all measurements. In all cases, the volume weighted particle size distributions, $\mathrm{D}_{10}, \mathrm{D}_{50}$, and $\mathrm{D}_{90}$ (where for example $\mathrm{D}_{50}$ is the maximum particle diameter below which $50 \%$ of the sample volume exists-also known as the median particle size by volume) were determined and evaluated. The specific surface area was derived from the particle size distribution data. The assumption was made that all the measured particles were spherical.

\subsubsection{Preparation of Solid Products for Physical-Chemical Investigations of Products}

After the particle size determination, the selected pre-dispersions were dried in a vacuum dryer (Binder $\mathrm{GmbH}$, Tuttlingen, Germany) at $40^{\circ} \mathrm{C}$ in order to obtain solid products for physicochemical investigations. The abbreviations of solid state samples are summarized in Table 5.

Table 5. Sample abbreviations.

\begin{tabular}{cccc}
\hline Sample Name & MEL (\%) & PVA (\%) & Description \\
\hline MEL_raw & 100 & - & untreated MEL \\
MEL_PVA_pm & 80 & 20 & physical mixture of MEL and PVA \\
MEL_W 10 min & 100 & - & dried MEL, milled for 10 min in water \\
MEL_W 50 min & 100 & - & dried MEL, milled for 50 min in water \\
MEL_W 60 min & 100 & - & dried MEL, milled for 60 min in water \\
MEL_W 90 min & 100 & - & dried MEL, milled for 90 min in water \\
MEL_W_PVA 10 min & 80 & 20 & dried product of milling for 10 min in PVA-solution \\
MEL_W_PVA 50 min & 80 & 20 & dried product of milling for 50 min in PVA-solution \\
MEL_W_PVA 60 min & 80 & 20 & dried product of milling for 60 min in PVA-solution \\
MEL_W_PVA 90 min & 80 & 20 & dried product of milling for 90 min in PVA-solution \\
\hline
\end{tabular}

\subsubsection{Further Investigations of the Products}

Structural Analysis

The physical state of the MEL in the samples was evaluated by XRPD. XRPD patterns were produced with a Bruker D8 Advance diffractometer (Bruker AXS GmbH, Karlsruhe, Germany) system with $\mathrm{Cu} \mathrm{K} \lambda \mathrm{I}$ radiation $(\lambda=1.5406 \AA)$. The samples were scanned at $40 \mathrm{kV}$ and $40 \mathrm{~mA}$ from $3^{\circ}$ to $40^{\circ} 2 \theta$, at a scanning speed of $0.05^{\circ} / \mathrm{s}$ and a step size of $0.010^{\circ}$. The crystallinity $\left(X_{c}\right)$ of the MEL in dried pre-dispersions, milled for 10, 50, 60 and 90 min was determined semi-quantitatively in case of both dispersant medium via the mean of the decrease of the total area beneath the curve of 3 characteristic peaks $\left(A_{\text {crystalline }}\right)$ compared to the raw MEL and MEL-poly(vinyl alcohol) physical mixture (MEL_PVA_pm) $\left(A_{\text {crystalline }}+A_{\text {amorphous }}\right)$ :

$$
X_{c}=\frac{A_{\text {crystalline }}}{A_{\text {crystalline }}+A_{\text {amorphous }}} \times 100
$$

DSC measurements were carried out with a Mettler Toledo DSC $821^{\mathrm{e}}$ thermal analysis system equipped with the STAR ${ }^{\mathrm{e}}$ thermal analysis program V9.0 (Mettler Inc., Schwerzenbach, Switzerland). Approximately 2-5 mg of pure drug or product was examined in the temperature range between $25^{\circ} \mathrm{C}$ and $300^{\circ} \mathrm{C}$. The heating rate was $5^{\circ} \mathrm{C} \cdot \mathrm{min}^{-1}$. Argon was used as carrier gas at a flow rate of $10 \mathrm{~L} \cdot \mathrm{h}^{-1}$ during the DSC investigations.

\section{Image Analysis (SEM)}

The shape and surface characteristics of the samples were visualized by using a scanning electron microscope (Hitachi S4700, Hitachi Scientific Ltd., Tokyo, Japan). The samples were sputter-coated with gold-palladium under an argon atmosphere, using a gold sputter module in a high-vacuum evaporator and the samples were examined at $15 \mathrm{kV}$ and $10 \mu \mathrm{A}$. The air pressure was 1.3-13 MPa. The milling-produced suspensions were dried in order to obtain solid products for SEM analyses. 


\section{In Vitro Release}

The paddle method with the USP dissolution apparatus (USP dissolution apparatus, type II Pharma Test, Heinburg, Germany) was used to examine MEL (1.67 mg), MEL_PVA_pm and the products $(2.0875 \mathrm{mg})$. The medium was $100 \mathrm{~mL}$ of phosphate buffer of $\mathrm{pH} 7.4 \pm 0.1$. The basket was rotated at $100 \mathrm{rpm}$ and sampling was performed up to $120 \mathrm{~min}$. After filtration (filtration pore size $0.22 \mu \mathrm{m}$; applying a Millex-HV syringe-driven filter unit, Millipore Corporation, Bedford, MA, USA) and dilution, the MEL contents of the samples were determined by spectrophotometry (ATI-UNICAM UV/VIS Spectrophotometer, Cambridge, UK) at $362 \mathrm{~nm}$.

\section{Conclusions}

A combination of planetary ball and pearl milling (using pearls as milling media) can be applied as a wet milling procedure to decrease the particle size and change the crystal morphology of MEL. Wet milling in aqueous medium adheres to green technology principles as the product does not contain organic solvent residues. Besides several advantages of wet milling, it is necessary to calculate the wear of pearls during milling.

During our work at constant rotation rate in the presence and absence of a stabilizer, the effects of milling time on the particle size reduction was determined (Table 6). Without additive, in the case of water-containing samples micronization could be achieved $\left(D_{50}=3.55 \mu \mathrm{m}\right)$. In the presence of PVA, depending on the milling time, the particle size of the drug could be reduced to the micro-(after $10 \mathrm{~min}),\left(\mathrm{D}_{50}=2.96 \mu \mathrm{m}\right)$ or nanometre range (after $\left.50 \mathrm{~min}, \mathrm{D}_{50}=126 \mathrm{~nm}\right)$. The effect of milling on the crystallinity of MEL was investigated. XRPD and DSC examinations revealed a decrease in the crystallinity of MEL. In the case of water-containing samples (without PVA) aggregation occurred during the course of milling. In the PVA-containing samples amorphization was determined (the degree of MEL crystallinity was $2 \%$ at the end of the milling at $90 \mathrm{~min}$ ). SEM images revealed the aggregation of nanosized particles in water-containing samples. In the presence of additive milling for $10 \mathrm{~min}$ resulted in irregularly shaped particles. The nanonized MEL crystals exhibited a regular shape and smooth surface. The in vitro dissolution tests showed that the reduction of the particle size of MEL, the increased SSA and the structural transformation of drug resulted in a rapid dissolution in case of nanonized MEL-containing product. The amorphous form of the drug does not require lattice energy to break the bonds during the dissolution process as in the crystalline state case.

Table 6. Summarized information about the determined properties of the samples.

\begin{tabular}{cccc}
\hline Sample & D $_{\mathbf{5 0}}(\mu \mathrm{m})$ & Crystallinity (\%) & Dissolved MEL at 30 min (\%) \\
\hline MEL_raw & 34.26 & 100 & 8.53 \\
MEL_PVA_pm & 20.15 & 80 & 29.22 \\
MEL_W 10 min & 3.55 & 3 & 37.82 \\
MEL_W_PVA 10 min & 2.96 & 48 & 64.73 \\
MEL_W_PVA 50 min & 0.126 & 12 & 82.16 \\
\hline
\end{tabular}

The combined wet milling technology was suitable for preparation of micronized MEL without the use of stabilizer and, depending on the milling time, of micronized and nanonized drug particles-containing pre-dispersions in the presence of PVA. Milling in the presence of additive could be the first step of pre-formulation and further formulation procedures. Decreased particle size (especially accessing the nanosize range) and the amorphization of drug could ensure higher dissolution rate and better bioavailability of poorly-water soluble drugs, though instability problems could occur in the case of amorphous forms of materials. To check the stability of the systems further investigations are needed. 
Because of the low need for dispersant medium, the combined method can be used for efficient milling, and it is also suggested for the preparation of the pre-dispersions with micro- and nanosized particles, and recommended for the development of particle size-controlled therapeutic systems.

Acknowledgments: This project was supported by the János Bolyai Research Scholarship of the Hungarian Academy of Sciences (2014-2017). The work was supported by the Gedeon Richter's Talentum Foundation (Budapest).

Author Contributions: Piroska Szabó-Révész, Rita Ambrus, Csilla Bartos and Csaba Bartos designed research. Rita Ambrus, Orsolya Jójárt-Laczkovich, Gábor Katona and Csilla Bartos analysed results and wrote the manuscript. Rita Ambrus and Piroska Szabó-Révész controlled the text. All authors contributed to the paper and approved the manuscript.

Conflicts of Interest: The authors declare no conflict of interest.

\section{References}

1. Truong-Dinh Tran, T.; Tran, K.A.; Ha-Lien Tran, P. Modulation of particle size and molecular interactions by sonoprecipitation method for enhancing dissolution rate of poorly water-soluble drug. Ultrason. Sonochem. 2015, 24, 256-263. [CrossRef] [PubMed]

2. Mansouri, S.; Kralj, T.P.; Morton, D.; Chen, X.D.; Woo, M.W. Squeezing out ultrafine hydrophobic and poor water-soluble drug particles with water vapour. Adv. Powder Technol. 2014, 25, 1190-1194. [CrossRef]

3. Caliandro, R.; di Profio, G.; Nicolotti, O. Multivariate analysis of quaternary carbamazepine-saccharin mixtures by X-ray diffraction and infrared spectroscopy. J. Pharm. Biomed. Anal. 2013, 78-79, 269-279. [CrossRef] [PubMed]

4. Miclea, L.M.; Vlaia, L.; Vlaia, V.; D.I. Hădărugă, C. Mircioiu, Preparation and characterization of inclusion complexes of meloxicam and $\alpha$-cyclodextrin and $\beta$-cyclodextrin. Farmacia 2010, 58, 583-593.

5. Verma, S.; Gokhale, R.; Burgess, D.J. A comparative study of top-down and bottom-up approaches for the preparation of micro/nanosuspensions. Int. J. Pharm. 2009, 380, 216-222. [CrossRef] [PubMed]

6. Serajuddin, A.T.M. Salt formation to improve drug solubility. Adv. Drug Deliv. Rev. 2007, 59, $603-616$. [CrossRef] [PubMed]

7. Hassan, M.A.; Suleiman, M.S.; Najib, N.M. Improvement of the in vitro dissolution characteristics of famotidine by inclusion in $\beta$-cyclodextrin. Int. J. Pharm. 1990, 58, 19-24. [CrossRef]

8. Paulino, A.S.; Rauber, G.; Campos, C.E.M.; Maurício, M.H.P.; de Avillez, R.R.; Capobianco, G.; Cardoso, S.G.; Cuffini, S.L. Dissolution enhancement of deflazacort using hollow crystals prepared by antisolvent crystallization process. Eur. J. Pharm. Sci. 2013, 49, 294-301. [CrossRef] [PubMed]

9. Lim, R.T.Y.; Ng, W.K.; Tan, R.B.H. Dissolution enhancement of indomethacin via amorphization using co-milling and supercritical co-precipitation processing. Powder Technol. 2013, 240, 79-87. [CrossRef]

10. Liu, P.; Rong, X.; Laru, J.; van Veen, B.; Kiesvaara, J.; Hirvonen, J.; Laaksonen, T.; Peltonen, L. Nanosuspensions of poorly soluble drugs: Preparation and development by wet milling. Int. J. Pharm. 2011, 411, 215-222. [CrossRef] [PubMed]

11. Noyes, A.A.; Whitney, W.R. The rate of solution of solid substances in their own solutions. J. Am. Chem. Soc. 1897, 19, 930-934. [CrossRef]

12. Manfredini \& Schianchi. MS DRYTECH: Continuous Evolution in the Dry Preparation of Raw Materials. Available online: http://www.manfredinieschianchi.com/406-2EN-advantages-of-dry-grindiing.htm (accessed on 20 March 2016).

13. Swarbrick, J. Encyclopedia of Pharmaceutical Technology, 3rd ed.; Informa Healthcare: New York, NY, USA, 2013; Volume 6.

14. Merisko-Liversidge, E.; Liversidge, G.G. Nanosizing for oral and parenteral drug delivery: A perspective on formulating poorly-water soluble compounds using wet media milling technology. Adv. Drug. Deliv. Rev. 2011, 63, 427-440. [CrossRef] [PubMed]

15. Blagden, N.; Matas, M.; Gavan, P.T.; York, P. Crystal engineering of active pharmaceutical ingredients to improve solubility and dissolution rates. Adv. Drug Deliv. Rev. 2007, 59, 617-630. [CrossRef] [PubMed]

16. Bund, R.K.; Pandit, A.B. Sonocrystallization: Effect on lactose recovery and crystal habit. Ultrason. Sonochem. 2007, 14, 143-152. [CrossRef] [PubMed] 
17. Bakar, M.R.A.; Nagy, Z.K.; Saleemi, A.N.; Rielly, C.D. The impact of direct nucleation control on crystal size distribution in pharmaceutical crystallization processes. Cryst. Growth Des. 2009, 9, 1378-1384. [CrossRef]

18. Salazar, J.; Ghanem, A.; Müller, R.H.; Möschwitzer, J.P. Nanocrystals: Comparison of the size reduction effectiveness of a novel combinative method with conventional top-down approaches. Eur. J. Pharm. Biopharm. 2012, 81, 82-90. [CrossRef] [PubMed]

19. Möschwitzer, J.P. Drug nanocrystals in the commercial pharmaceutical development process. Int. J. Pharm. 2013, 453, 142-156. [CrossRef] [PubMed]

20. Direct Industry, Retsch ${ }^{\circledR}$ All Retsch Catalogues and Technical Brochures. Available online: http:/ / pdf.directindustry.com/pdf/retsch/the-sample-high-energy-ball-mills/19308-518973.html (accessed on 20 March 2016).

21. Paltonen, L.; Hirvonen, J. Pharmaceutical nanocrystals by nanomilling: Critical process parameters, particle fracturing and stabilization methods. J. Pharm. Pharmacol. 2010, 62, 1569-1579. [CrossRef] [PubMed]

22. Smith, G.; Hussain, A.; Bukhari, N.I.; Ermolina, I. Quantification of residual crystallinity in ball milled commercially sourced lactose monohydrate by thermo-analytical techniques and terahertz spectroscopy. Eur. J. Pharm. Biopharm. 2015, 92, 180-191. [CrossRef] [PubMed]

23. Mártha, C.; Kürti, L.; Farkas, G.; Jójárt-Laczkovich, O.; Szalontai, B.; Glässer, E.; Deli, M.A.; Szabó-Révész, P. Effects of polymers on the crystallinity of nanonized meloxicam during a co-grinding process. Eur. Polym. J. 2013, 49, 2426-2432. [CrossRef]

24. Pan, X.; Julian, T.; Augsburger, L. Increasing the dissolution rate of a low-solubility drug through a crystalline-amorphous transition: A case study with indomethacin. Drug Dev. Ind. Pharm. 2008, 34, 221-231. [CrossRef] [PubMed]

25. Ambrus, R.; Kocbek, P.; Kristl, J.; Šibanc, R.; Rajkó, R.; Szabó-Révész, P. Investigation of preparation parameters to improve the dissolution of poorly water-soluble meloxicam. Int. J. Pharm. 2009, 381, 153-159. [CrossRef] [PubMed]

26. Hughey, J.R.; Keen, J.M.; Brough, C.; Saeger, S.; McGinity, J.W. Thermal processing of a poorly water-soluble drug substance exhibiting a high melting point: The utility of KinetiSol ${ }^{\circledR}$ Dispersing. Int. J. Pharm. 2011, 419, 222-230. [CrossRef] [PubMed]

Sample Availability: Not available.

(C) 2016 by the authors; licensee MDPI, Basel, Switzerland. This article is an open access article distributed under the terms and conditions of the Creative Commons Attribution (CC-BY) license (http:/ / creativecommons.org/licenses/by/4.0/). 


\section{PUBLICATION II.}




\section{Optimization of a combined wet milling process in order to produce poly(vinyl alcohol) stabilized nanosuspension}

This article was published in the following Dove Press journal: Drug Design, Development and Therapy

\section{Csaba Bartos' \\ Orsolya Jójárt-Laczkovich' Gábor Katona' \\ Mária Budai-Szűcs' \\ Rita Ambrus' \\ Alexandra Bocsik ${ }^{2}$ \\ Ilona Gróf ${ }^{2}$ \\ Mária Deli² \\ Piroska Szabó-Révész' \\ 'Faculty of Pharmacy, Institute of Pharmaceutical Technology and Regulatory Affairs, University of Szeged, Szeged, Hungary; ${ }^{2}$ Institute of Biophysics, Biological Research Centre, Hungarian Academy of Sciences, Szeged, Hungary}

Correspondence: Piroska Szabó-Révész Faculty of Pharmacy, Institute of Pharmaceutical Technology and Regulatory Affairs, University of Szeged, Eötvös u 6, Szeged H-6720, Hungary Tel +36 62545572

Fax +36 62545571

Email revesz@pharm.u-szeged.hu
Abstract: The paper reports a wet milling process, where the planetary ball mill was combined with pearl milling technology to produce nanosize meloxicam (Mel). Mel as a water-insoluble highly potent nonsteroidal anti-inflammatory drug was milled in the presence of different amounts of the stabilizer agent, poly(vinyl alcohol) (PVA), in aqueous solution. The pearl amount, the milling time, and the rotation speed of the jar were optimized to reach the particle size range of $100-500 \mathrm{~nm}$. The novelty of this study is the optimization of PVA amount in order to reach the required particle size distribution of $\mathrm{Mel}$ (milling efficiency) and the investigation of the effect of PVA concentration on the physicochemical properties of the milled dispersion (viscosity, zeta potential) to predict the stability of the nanosuspensions. It was found that the ratio of predispersion and pearls $1: 1(\mathrm{w} / \mathrm{w})$ resulted in the most effective grinding system (200-fold particle size reduction in 1 step) with optimized process parameters, $437 \mathrm{rpm}$ and $43 \mathrm{~min}$. Nanosuspension ( $1 \% \mathrm{Mel}$ and $0.5 \% \mathrm{PVA})$ as an intermediate product showed a stable system with 2 weeks of holding time. This optimized nanosuspension enhanced the penetration of Mel across cultured intestinal epithelial cell layers without toxic effects. The dissolution rate of Mel from the PVA-stabilized nanosuspension justified its applicability in the design of innovative per oral dosage form (capsule) in order to ensure/give a rapid analgesia.

Keywords: nanonization, meloxicam, milled dispersion, milling efficiency, zeta potential, intermediate product

\section{Introduction}

The planetary ball milling belongs to the group of high-energy milling methods. The process is mainly used in laboratory-scale research work. It is a common technique for dry milling, ${ }^{1,2}$ nevertheless it is also suitable for wet grind..$^{3-5}$ Dry milling with this technique is usually used for micronization with a particle size range of $1-2,000 \mu \mathrm{m} .{ }^{6}$ In general, additives are not required for micronization, but for dry nanonization, application of them and a long milling time $(2-4 \mathrm{~h})$ can be necessary. Additives decrease the cohesion between the nanosize particles and the collision energy during the milling process; thereby, the risk of the decomposition of the active agent can be reduced.?

Wet milling is a top-down process, where the raw material is broken down via mechanical forces. In this method, a sufficiently concentrated dispersion of drug particles in an aqueous or nonaqueous liquid medium is treated. Increased mill capacity, lower energy consumption, and easier handling of materials can be perceived as advantages of the process. However, it must be said, in the course of the milling process, increased wear of the milling medium and corrosion can occur, and the 
instability of the active agent must be taken into account. Wet milling is applicable for micronization in the particle size range of $1-50 \mu \mathrm{m}$, in this case, colloid mill, toothed high-shear inline mixers, and cone mill can be used, ${ }^{8}$ but for nanonization, high-pressure homogenization (1-20 $\mu \mathrm{m})$ and pearl milling technique $(20-200 \mathrm{~nm})^{9-11}$ can be applied. It should be noted that in case of high-pressure homogenization and pearl milling techniques, the preparation of pretreated dispersions (particle size reduction to $1-10 \mu \mathrm{m}$ ) is required to reach the nanosize range.

The pearl milling process has been proven to be a robust technique for the production of nanoparticle suspension of poorly water-soluble drugs. ${ }^{12}$ With this method, nanosuspensions are produced through the use of high-shear media or pearl mills. Pearl milling is a continuous process wherein the drug suspension is pumped through the milling chamber in order to reduce the particle size of the suspended material. The milling medium consists of glass, zirconia, or highly cross-linked polystyrene resin. ${ }^{13}$ The physical characteristics of the resulting nanocrystals depend on the number and size of the milling pearls, the amount of the drug, and the stabilizer(s). ${ }^{14,15}$

Thanks to the high efficiency of a smaller pearl size ${ }^{16}$ and the high mechanical forces of the planetary mill, Retsch $\mathrm{GmbH}$ (Haan, Germany) recommends the combination of the planetary ball and pearl milling as a novel milling technique in order to prepare drug nanodispersions. ${ }^{17}$ In the literature, there are few articles about the combinative method, where various active agents were co-milled. In the presence of d-tocopherol polyethylene glycol 1000 succinate as a stabilizer agent, nanoparticle range was achieved; however, the production efficiency was very low. ${ }^{18,19}$

Our team uses different milling techniques (dry and wet) in order to nanonize different water-insoluble drugs, eg, meloxicam (Mel), to provide a faster dissolution, a higher saturated concentration, a faster absorption, and, in this context, a better bioavailability. Mel as a nonsteroidal antiinflammatory agent is often used in malignant and nonmalignant pain therapy, but its bioavailability is unsatisfactory thanks to its poor solubility in the gastrointestinal tract. One strategy to address these problems is the particle size reduction (eg, nanonization), which increases the dissolution rate of the poorly soluble drug resulting in faster absorption and faster action in pain therapy.

In our earlier studies, we investigated the applicability of the combined wet milling technique, ${ }^{20-23}$ and these preliminary studies showed that for the nanonization of Mel, PVA as a stabilizer agent and an increased milling time ( $>50 \mathrm{~min})$ were required. The influence of milling time was also studied on the particle size distribution, crystallinity, and dissolution rate of $\mathrm{Mel}$.

During the use of the combined wet milling technique, the type and amount of PVA are very important because it has a dual role. On the one hand, it promotes the grinding efficiency in the concentrated predispersion, and on the other hand, it stabilizes the milled dispersion and later the final nanosuspension in a diluted medium.

PVA is a nonionic polymer with very different molecular weights. It is frequently used as a stabilizer agent. ${ }^{20}$ Polymer adsorption on the solid-liquid interface can be influenced by the various conformations of the polymer chains and the interaction of the polymer segments with the solvent and the surface of the solid as well. PVA with low molecular weight (about 20,000 $\mathrm{g} / \mathrm{mol}$ ) is adsorbed on the colloid particles and thus stabilizes the colloid suspension (coating the particles and providing the repulsion among them), whereas PVA with high molecular weight (about 1,000,000 g/mol) flocculates the dispersed systems. ${ }^{24}$

The prediction of permeability features of drug candidates across biological barriers is of great importance in the early phase of drug development. ${ }^{25}$ Oral drug formulations are the most widespread in human therapy, and therefore intestinal drug absorption is the most studied in pharmaceutical research. The human Caco-2 cell line, presenting many of the structural and functional aspects of the epithelium of small intestine, is a routinely used culture model of intestinal drug penetration showing good correlation with in vivo data. ${ }^{26}$

The aim of this work was to optimize the process parameters (pearl amount, milling time, and rotation speed) of the combined wet milling technique (planetary ball and pearl milling), using Mel as an active agent and PVA as a stabilizing agent in the predispersion. We basically investigated the influence of the amount of low molecular weight PVA on the grinding efficiency in concentrated predispersion. Our aim was also to get to know the effect of mechanical forces on polymer viscosity and drug-polymer interaction as well. To describe the stability of nanosuspensions, their particle size was monitored for 2 weeks. Furthermore, the optimized Mel formulations were tested on the cell culture model of intestinal epithelium.

The novelty of this study is the application of PVA and the combined wet milling process and optimization of the amount of the additive and the process parameters in order to produce Mel nanosuspension (particle size range of $100-500 \mathrm{~nm}$ ) without any pretreating procedure. 


\section{Materials and methods Materials}

Mel was obtained from EGIS Ltd. (Budapest, Hungary). PVA-Mowiol 4-98 ( $M_{\mathrm{w}}$ 27,000) (Sigma-Aldrich Co. LLC, St Louis, MO, USA) was used as a stabilizing agent. Reagents were purchased also from Sigma-Aldrich. Zirconium oxide $\left(\mathrm{ZrO}_{2}\right)$ beds with a diameter of $0.3 \mathrm{~mm}$ were obtained from Netsch (Netsch GmbH, Selb, Germany). All reagents were purchased from Sigma-Aldrich, Ltd. (Budapest, Hungary) for the in vitro cell culture experiments, unless otherwise indicated.

\section{Methods}

\section{Combined wet media milling} Optimization of process parameters

The samples were milled with the steel jar with $50 \mathrm{~mL}$ volume of the Retsch PM 100 planetary ball mill (Retsch PM 100 MA, Retsch $\mathrm{GmbH}$ ) combined with $0.3 \mathrm{~mm} \mathrm{ZrO}_{2}$ beads as the grinding media. The concentrated $(10 \% \mathrm{w} / \mathrm{w})$ predispersions ( $2 \mathrm{~g}$ of $\mathrm{Mel}$ suspended in $18 \mathrm{~g}$ of dispersant medium containing PVA) were added to the $\mathrm{ZrO}_{2}$ beads. In the first step, the effect of different amount of $\mathrm{ZnO}_{2}$ pearls on the particle size reduction was investigated. The ratio of the amount of predispersion and pearls $(\mathrm{w} / \mathrm{w})$ was 1:0.5, 1:1, $1: 2$, and 1:4; and the milling times were 10, 30, and $50 \mathrm{~min}$. In these cases, the concentration of PVA solution was $2.5 \%$ $(\mathrm{w} / \mathrm{w})$, and the rotation speed was $400 \mathrm{rpm}$. In the second step, design and analysis of experiments with 3 levels were used to optimize the milling time (10,30, and $50 \mathrm{~min})$ and the rotation speed $(200,350$, and $500 \mathrm{rpm})$ as independent variables. The amount of the pearls was chosen on the basis of the optimization study. The temperature of the samples was measured immediately after milling. This value did not exceed $39^{\circ} \mathrm{C}$.

\section{Optimization of PVA concentration}

Various amounts of PVA $(2.5 \%-7.5 \%)$ were applied to prepare the concentrated predispersions. The concentration of the PVA solutions was increased in the half percent range. Mel content was $10 \%(\mathrm{w} / \mathrm{w})$, and the optimized process parameters were used during the milling. The degree of particle size reduction and particle size distribution were the main factors for the optimization of the PVA concentration (Figure 1).

The concentrated milled dispersions were filtered by a sieve with $150 \mu \mathrm{m}$ mesh size in order to remove the pearls. For the washing of the pearls, $180 \mathrm{~g}$ of distilled water was used. In all cases, the milled dispersions were 10-fold diluted

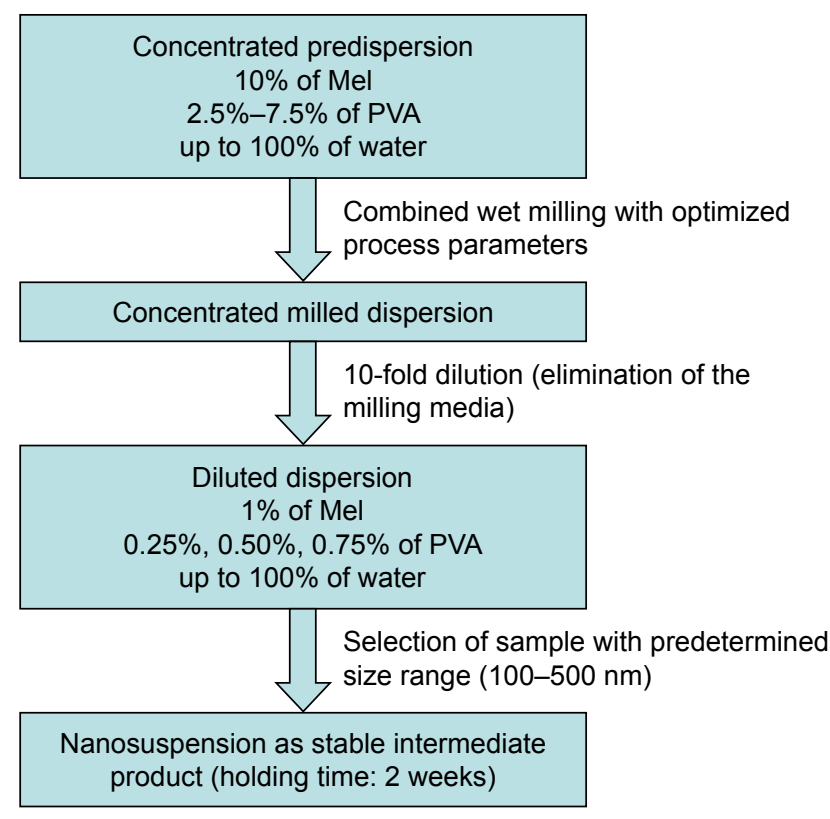

Figure I Protocol of sample preparation for the optimization of PVA content. Abbreviations: Mel, meloxicam; PVA, poly(vinyl alcohol).

products with $1 \%$ of $\mathrm{Mel}(\mathrm{w} / \mathrm{w})$ content. The samples were selected on the basis of the particle size range $(100-500 \mathrm{~nm})$ and the holding time (2 weeks).

\section{Investigation of the samples}

Particle size measurement

The volume-based particle size distribution was measured by laser diffraction (Mastersizer S 2000, Malvern Instruments Ltd, Worcestershire, UK) with the following parameters: 300RF lens; small volume dispersion unit (1,000 rpm); refractive index for dispersed particles 1.596; and refractive index for dispersion medium 1.330. Water was used as a dispersant, and the obscuration was in the range of $11 \%-16 \%$ for all measurements. In all cases, the particle size distributions were characterized by the $d(0.1), d(0.5)$, and $d(0.9)$ (where, eg, $d(0.5)$ is the maximum particle diameter below which $50 \%$ of the sample volume exists), and the Span values were calculated according to equation. A high Span value $(>1)$ denotes a broad particle size distribution. ${ }^{27}$

$$
\text { Span }=\frac{d(0.9)-d(0.1)}{d(0.5)}
$$

\section{Rheological measurement}

To investigate the viscosity changes during the milling process, the initial and milled PVA solutions and the concentrated milled dispersions were used. Rheological measurements were carried out with Physica MCR101 rheometer 
(Anton Paar, Graz, Austria). A concentric cylinder measuring device with a diameter of $17 \mathrm{~mm}$ was used. The flow curves of the samples were determined at $25^{\circ} \mathrm{C}$, where the shear rate was increased from 0.1 to $100 \mathrm{~L} / \mathrm{s}$. The shearing time was $300 \mathrm{sec}$. In this paper, viscosity values were given at $100 \mathrm{~L} / \mathrm{s}$ shear rate. Two parallel measurements were run.

\section{Zeta potential}

The zeta potential of the dispersions was measured using a Malvern Zeta Nano ZS (Malvern Instruments Ltd). For the zeta potential determination, Malvern DTS 1070 folded capillary cell was used. The diluted milled dispersions were further diluted with water (25-fold) for the measurements.

\section{Raman spectroscopy}

For the investigation of Mel degradation as a function of the pearl amount and milling time in the dispersion, Raman spectra were acquired with a Thermo Fisher DXR Dispersive Raman (Thermo Fisher Scientific Inc., Waltham, MA, USA) equipped with a CCD camera and a diode laser operating at a wavelength of $532 \mathrm{~nm}$. Raman measurements were carried out with a laser power of 4 and $8 \mathrm{~mW}$ at $25-\mu \mathrm{m}$ slit aperture size on a $2 \mu \mathrm{m}$ spot size. The spectra of the individual substances as Mel and PVA were collected using a 2 -sec exposure time, a total of 48 scanning in the spectral range of 3,300-200 $\mathrm{cm}^{-1}$ with cosmic ray and fluorescence corrections.

\section{Morphology of the particles (scanning electron microscopy)}

For the investigation of the morphology of the particles, the diluted milled dispersions were dried in a vacuum dryer (Binder $\mathrm{GmbH}$, Tuttlingen, Germany) at $40^{\circ} \mathrm{C}$ in order to obtain solid products for physicochemical investigations. After drying, the shape and surface characteristics of the samples were visualized using a scanning electron microscope (Hitachi S4700, Hitachi Scientific Ltd., Tokyo, Japan). The samples were sputter-coated with gold-palladium under an argon atmosphere, using a gold sputter module in a highvacuum evaporator, and the samples were examined at $10 \mathrm{kV}$ and $10 \mathrm{~mA}$. The air pressure was $1.3-13 \mathrm{MPa}$.

\section{Differential scanning calorimetry (DSC)}

DSC measurements were carried out with a Mettler Toledo DSC $821^{\mathrm{e}}$ thermal analysis system with the $\mathrm{STAR}^{\mathrm{e}}$ thermal analysis software V9.0 (Mettler Inc., Schwerzenbach, Switzerland). Approximately 2-5 mg of pure Mel and PVA as well as dried samples were examined in the temperature range of $25^{\circ} \mathrm{C}-270^{\circ} \mathrm{C}$. The heating rate was $10^{\circ} \mathrm{C} / \mathrm{min}$ in the presence of argon as a carrier gas with a flow rate of $10 \mathrm{~L} / \mathrm{h}$.

\section{X-ray powder diffraction analysis (XRPD)}

The crystallinity state of Mel in the dried samples was evaluated by XRPD. XRPD patterns were produced by a Bruker D8 Advance diffractometer (Bruker AXS GmbH, Karlsruhe, Germany) system with $\mathrm{Cu} \mathrm{K} \lambda \mathrm{I}$ radiation $(\lambda=1.5406 \AA)$. The samples were scanned at $40 \mathrm{kV}$ and $40 \mathrm{~mA}$ from 3 to $402 \theta$, at a step time of $0.1 \mathrm{sec}$, and a step size of $0.010^{\circ}$. The instrument was calibrated by using SI standard. The semiquantitative determination of Mel crystallinity (Cryst. \%) was performed using the total area under the curve of 3 characteristic peaks $(13.06,14.94$, and 18.61 $2 \theta$ ) of Mel. The area under the curve value of the peak of raw material without milling (rawMel) and the dried milled dispersions (MelD) was calculated and compared according the following formula:

$$
\text { Cryst. } \%=\frac{\text { AUCMelD }}{\text { AUCrawMel }} \times 100
$$

\section{Drug content determination}

The loss of weight of Mel was controlled in the milled suspension. Seventy-five milligram of the liquid products with $0.75 \mathrm{mg}$ of theoretical Mel was dissolved in $100 \mathrm{~mL}$ of phosphate buffer $\mathrm{pH} 7.4 \pm 0.1$. The sample was stirred with a magnetic stirrer at $25^{\circ} \mathrm{C}$ for $24 \mathrm{~h}$ and then filtered $(0.1 \mu \mathrm{m}$, FilterBio PES Syringe Filter) (Labex Ltd., Budapest, Hungary), and the concentration of the dissolved Mel was analyzed spectrophotometrically (Unicam UV/VIS) (Thermo Fisher Scientific Inc.) at $364 \mathrm{~nm}$ wavelength. The investigations were repeated 3 times.

\section{In vitro dissolution test}

To determine the dissolution extent of Mel from dispersions, the paddle method (USP dissolution apparatus, type II Pharma Test, Heinburg, Germany) was used. About $750 \mathrm{mg}$ of the dispersion with $7.5 \mathrm{mg}$ of Mel (therapeutic dose) was filled into hard gelatin capsules within $5 \mathrm{sec}$ and put promptly into the medium. The medium contained $900 \mathrm{~mL}$ of artificial gastric fluid at $\mathrm{pH} 1.2 \pm 0.1$ and intestinal fluid $(\mathrm{pH} 6.8 \pm 0.1)$. The paddle was rotated at $100 \mathrm{rpm}$, and sampling was performed up to $60 \mathrm{~min}$. The Mel contents of the samples were determined using a spectrophotometer (ATI-UNICAM UV/VIS Spectrophotometer) at $362 \mathrm{~nm}$ (gastric juice) and $364 \mathrm{~nm}$ (enteric fluid). The number of parallel runs was 3. 


\section{In vitro cell culture studies}

\section{Human Caco-2 intestinal epithelial cell line}

Caco-2 intestinal epithelial cell line was purchased from ATCC (Manassas, VA, USA) (cat. no HTB-37) and used until passage 60 for the experiments. The cells were grown in DMEM (Gibco, Life Technologies, Carlsbad, CA, USA) and supplemented with 10\% fetal bovine serum (Pan-Biotech $\mathrm{GmbH}$, Aidenbach, Germany) and $50 \mu \mathrm{g} / \mathrm{mL}$ gentamycin in a humidified incubator with $5 \% \mathrm{CO}_{2}$ at $37^{\circ} \mathrm{C}$. All plastic surfaces were coated with $0.05 \%$ rat tail collagen in sterile distilled water before cell seeding in culture dishes and the medium was changed every 2 days. The stock solutions for cell culture experiments were the following: Mel, $100 \mathrm{mg}$ / $\mathrm{mL}$; PVA, 5\% (w/w), and 3 products containing $100 \mathrm{mg} / \mathrm{mL}$ Mel with various amounts of PVA $(2.5 \%, 5 \%$, or $7.5 \%)$. The working solutions were diluted in the cell culture medium or Ringer-Hepes buffer and contained $1 \mathrm{mg} / \mathrm{mL}$ of Mel for the $\mathrm{Mel}$ and formulation groups. The final concentrations of PVA were $0.025 \%, 0.05 \%$, and $0.075 \%(\mathrm{w} / \mathrm{w})$.

\section{Cell viability measurement by impedance}

Impedance was measured at $10 \mathrm{kHz}$ using the RTCA-SP instrument (RTCA-SP instrument, ACEA Biosciences, San Diego, CA, USA). This method is label-free, noninvasive and follows cell adherence, growth, number, and viability real time. We have successfully tested the cellular effects of peptides and pharmaceutical excipients by impedance kinetics. ${ }^{28-30}$ For background measurements, a $50 \mu \mathrm{L}$ cell culture medium was added to the wells; then, cells were seeded at a density of $6 \times 10^{3}$ cells/well to 96 -well plate with gold electrodes (E-plate 96, ACEA Biosciences) coated with collagen. Cells were cultured for 5 days in a $\mathrm{CO}_{2}$ incubator at $37^{\circ} \mathrm{C}$ and monitored every $10 \mathrm{~min}$ until the end of experiments. At the beginning, plateau phase of growth cells were treated with Mel, PVA, and Mel+PVA samples diluted in a cell culture medium, and the effects were followed for $8 \mathrm{~h}$. Triton X-100 detergent ( $1 \mathrm{mg} / \mathrm{mL})$ was used as a reference compound to induce cell toxicity. Cell index was defined as $R_{\mathrm{n}}-R_{\mathrm{b}}$ at each time point of measurement, where $R_{\mathrm{n}}$ is the cell-electrode impedance of the well when it contains cells and $R_{\mathrm{b}}$ is the background impedance of the well with the medium alone.

\section{Permeability study on cell culture model}

Transepithelial electrical resistance (TEER) reflects the tightness of the intercellular junctions closing the paracellular cleft, and therefore reflects the overall tightness of cell layers of biological barriers. TEER was measured every 2 days to check the barrier integrity by an EVOM volt-ohmmeter (World Precision Instruments, Sarasota, FL, USA) combined with STX-2 electrodes and was expressed relative to the surface area of the monolayers as $\Omega \times \mathrm{cm}^{2}$.

Caco- 2 cells were seeded onto Transwell inserts (polycarbonate membrane, $0.4 \mu \mathrm{m}$ pore size, $1.12 \mathrm{~cm}^{2}$ surface area; Corning Life Sciences, Tewksbury, MA, USA) and cultured for 3 weeks. ${ }^{31,32}$ The culture medium was changed and TEER was checked every second day.

For the permeability experiment, inserts were transferred to 12 -well plates containing $1.5 \mathrm{~mL}$ Ringer-Hepes buffer in the lower (basal) compartments. In the upper (apical) compartments, the culture medium was replaced by $0.5 \mathrm{~mL}$ buffer containing treatment solutions of Mel, PVA, and Mel formulation groups for $1 \mathrm{~h}$. Permeability marker molecules fluorescein $\left(10 \mu \mathrm{g} / \mathrm{mL} ; M_{\mathrm{w}}: 376 \mathrm{Da}\right)$ and albumin $(10 \mathrm{mg} / \mathrm{mL}$; $\left.M_{\mathrm{w}}: 65 \mathrm{kDa}\right)$ labeled with Evans blue $(167.5 \mu \mathrm{g} / \mathrm{mL})$ were used for verifying the cell layer integrity. Treatment solutions from both compartments were collected and the Mel level was detected using a Thermo Spectronic Helios Alpha UV-Vis spectrophotometer (Thermo Fisher Scientific Inc.).

The concentrations of the permeability marker molecules of collected samples were determined by a fluorescence multi-well plate reader (Fluostar Optima, BMG Labtech, Offenburg, Germany; for fluorescein: excitation wavelength, $485 \mathrm{~nm}$; emission wavelength, $535 \mathrm{~nm}$ and for Evans bluelabeled albumin: excitation wavelength, $584 \mathrm{~nm}$; emission wavelength, $680 \mathrm{~nm}$ ).

The apparent permeability coefficients $\left(P_{\text {app }}\right)$ were calculated as described previously. ${ }^{28}$ Briefly, the cleared volume was calculated from the concentration difference of the tracer in the lower/basal compartment $\left(\Delta[C]_{\mathrm{B}}\right)$ after $30 \mathrm{~min}$ and upper/ apical compartments at $0 \mathrm{~h}\left([\mathrm{C}]_{\mathrm{A}}\right)$, the volume of the lower/ basal compartment $\left(V_{\mathrm{B}}, 1.5 \mathrm{~mL}\right)$ and the surface area available for permeability $\left(A, 1.1 \mathrm{~cm}^{2}\right)$ using the following equation:

$$
P_{\text {app }}(\mathrm{cm} / \mathrm{s})=\frac{\Delta[C]_{\mathrm{A}} \times V_{\mathrm{A}}}{A \times[C]_{\mathrm{L}} \times \Delta t}
$$

\section{Determination of holding time}

Since the diluted dispersions are intermediate products, it was necessary to specify the "holding time" of the sample through the particle size distribution. The products were stored in sealed glass bottles at room temperature $\left(25^{\circ} \mathrm{C} \pm 1^{\circ} \mathrm{C}\right)$ for 2 weeks. The particle size distribution of the $\mathrm{Mel}$ in the samples was analyzed on the day of production (day 0 ) and after 14 days of storage. 


\section{Statistical analyses}

Data were expressed as mean $\pm \mathrm{SD}$, and groups were compared by using Student's $t$-test. For the evaluation of cell culture results, GraphPad Prism 5.0 software (GraphPad Software Inc., San Diego, CA, USA) was used. All culture data presented are mean $\pm \mathrm{SD}$; values were compared using analysis of variance followed by Bonferroni posttest. Differences were considered statistically significant when $p<0.05$.

\section{Results and discussion}

\section{Optimization of process parameters (pearl} amount, milling time, and rotation speed)

The effect of pearl amount and milling time on particle size of $d(0.5)$ was investigated. This study was performed using $2.5 \%$ PVA solution. ${ }^{23}$ The ratio of the concentrated predispersion (2.0 $\mathrm{g}$ of Mel+18.0 $\mathrm{g}$ of PVA aqueous solution) and pearl amount was different: 1:0.5, 1:1, 1:2, and 1:4(w/w). It can be established that the pearl amount and the milling time have a great effect of on the $d(0.5)$ value (Table 1$)$. The milling efficiency was not satisfactory in case of ratio $1: 0.5$, but it increased linearly on increasing the amount of the milling media and the milling time except in case of ratio 1:4. It was found that the increase of the amount of the milling media (up to 1:2 w/w or more) causes gray coloring because of the high friction between the pearls and the wall of the steel jar. Therefore, the pearl milling amount was optimized at the ratio of 1:1 (20 $\mathrm{g}$ of concentrated predispersion and $20 \mathrm{~g}$ of pearls), and the milling time was investigated as an independent variable in the factorial experimental design. Another advantage of the small amount of the grinding media may be the reduction of the product loss.

During the factorial experimental design, the influence of the milling time and the rotation speed on the particle size reduction was investigated. The ratio of the predispersion and the pearls was also $1: 1(\mathrm{w} / \mathrm{w})$. Figure 2 shows that the

Table I Particle size of Mel $(d[0.5])$ in milled dispersion as a function of different pearl amounts and milling time $(d[0.5]$ of raw Mel was $34.260 \pm 4.860 \mu \mathrm{m}$ )

\begin{tabular}{|c|c|c|c|c|}
\hline & \multicolumn{4}{|c|}{ Ratio of predispersion and pearl amount (w/w) } \\
\hline & $1: 0.5$ & I:I & $1: 2$ & I:4 \\
\hline & \multicolumn{4}{|c|}{ Particle size $(\mu \mathrm{m})$} \\
\hline $10 \mathrm{~min}$ & $4.015 \pm 0.06$ & $2.426 \pm 0.029$ & $2.383 \pm 0.016$ & $0.149 \pm 0.03$ \\
\hline $30 \mathrm{~min}$ & $0.293 \pm 0.008$ & $0.145 \pm 0.007$ & $0.190 \pm 0.003$ & $0.137 \pm 0.006$ \\
\hline $50 \mathrm{~min}$ & $0.202 \pm 0.003$ & $0.140 \pm 0.004$ & $0.140 \pm 0.002$ & $0.130 \pm 0.004$ \\
\hline
\end{tabular}

Abbreviations: Mel, meloxicam; $d(0.5)$, the maximum particle diameter below which $50 \%$ of the sample volume exists; w/w, weight/weight (concentration).

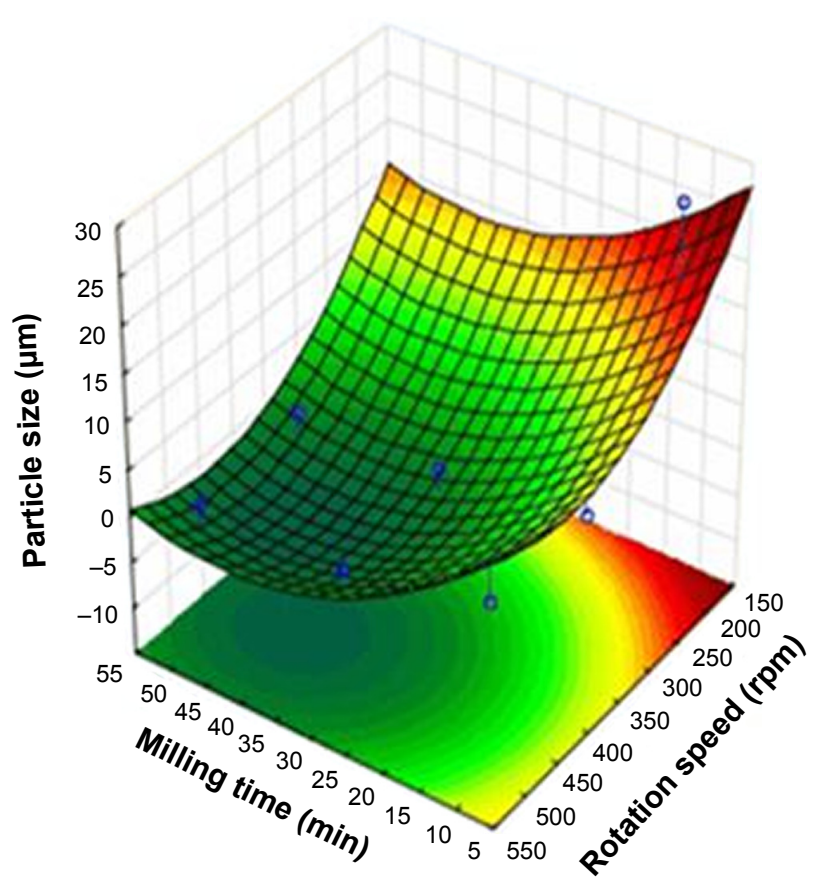

Figure 2 Three-dimensional illustration of the particle size changes during the second factorial experimental design.

efficiency of the particle size reduction was improved by increasing the milling time and the rotation speed. Based on the results, $437 \mathrm{rpm}$ and $43 \mathrm{~min}$ are the optimal parameters of the milling process.

\section{Optimization of PVA concentration} Influence of PVA amount on the milling effectiveness Concentrated predispersions with different PVA amounts $(2.5 \%-7.5 \%)$ were milled with optimized parameters ( $2 \mathrm{~g}$ of Mel+18 $\mathrm{g}$ of PVA aqueous solution, $20 \mathrm{~g}$ pearls, $437 \mathrm{rpm}, 43 \mathrm{~min}$ ). The results show (Figure 3) that the lower

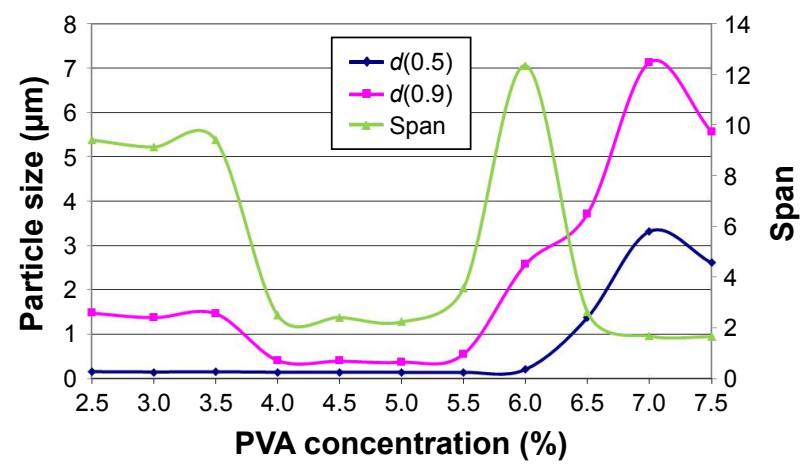

Figure 3 Particle size reduction effectiveness according to PVA concentrations and the Span values for the demonstration of particle size distribution $(d[0 . I]$ values are not shown).

Abbreviations: PVA, poly(vinyl alcohol); Span, calculated value for a broad particle size distribution; $d(0.1), d(0.5), d(0.9)$, the maximum particle diameter below which $10 \%, 50 \%$, and $90 \%$ of the sample volume exists. 
concentration of PVA (from $2.5 \%$ to $3.5 \%$ ) was not suitable to reach the nanosize range $(100-500 \mathrm{~nm})$. A higher concentration of PVA $(6.0 \%-6.5 \%)$ also resulted in unsatisfactory milling effectiveness. Over $6.5 \%$ a robust protecting layer was probably formed on the solid particles, and nanonization was not possible. The curve of the Span follows the different particle size distribution of the samples. The best particle size distribution was measured in the range of $4.0 \%$ and $5.5 \%$ of PVA. Based on the results, the concentrated milled dispersion containing $5.0 \%$ of PVA was selected as the optimized PVA amount.

\section{Influence of PVA amount on the physicochemical properties of milled dispersions}

After the optimization of the wet milling process and the PVA amount, in order to understand the influence of the amount of PVA on the physicochemical properties of the samples, the milled dispersions with 3 different concentrations of PVA were investigated. These were the following: $2.5 \%, 5.0 \%$ (as optimized), and $7.5 \%$ of PVA.

\section{Viscosity changes during the milling}

In order to exclude the viscosity changes because of the high mechanical forces during the milling, the viscosity of 3 raw PVA solutions $(2.5 \%, 5 \%$, and $7.5 \%)$ was investigated before the milling process, after the process ( $437 \mathrm{rpm}, 43 \mathrm{~min}$ ), and after the milling in addition with Mel. The polymer solutions showed Newtonian flow behavior as their shear viscosity was independent of the applied shear rate. The viscosity of the polymer solutions increased with increasing the polymer concentration (Table 2). There were no remarkable differences between the viscosity of the polymer solution before and after the milling procedure, which may indicate there are no changes in the polymer structure. $10 \% \mathrm{Mel}$ amount

Table 2 Viscosity values $(\eta)$ of the raw PVA solutions before milling (PVA \%) and after milling (PVA \% milled), and the PVA solutions after milling in addition with Mel (Mel PVA \% milled)

\begin{tabular}{lll}
\hline & $\eta, \mathbf{m P a}$ *s & SD \pm , mPa*s \\
\hline PVA 2.5\% & 3.14 & 0.02 \\
PVA 5.0\% & 7.38 & 0.02 \\
PVA 7.5\% & 20.55 & 0.07 \\
PVA 2.5\% milled & 3.12 & 0.27 \\
PVA 5.0\% milled & 7.09 & 0.02 \\
PVA 7.5\% milled & 20.05 & 0.07 \\
Mel PVA 2.5\% milled & 4.42 & 0.07 \\
Mel PVA 5.0\% milled & 8.46 & 0.01 \\
Mel PVA 7.5\% milled & 21.65 & 0.07 \\
\hline
\end{tabular}

Abbreviations: Mel, meloxicam; PVA, poly(vinyl alcohol); $\eta$, viscosity. in the dispersions increased the viscosity of the systems and did not change the flow behavior - they remained Newtonian. Adding Mel to the polymer solutions, the smallest shift in the viscosity could be detected in case of the highest polymer concentration. This can be explained by the more considerable viscosity changing effect of the polymer concentration than that of the Mel particles. It can be concluded that the mechanical influence did not change the viscosity of the polymer solutions, and therefore, the structure of the PVA chains did not change.

\section{Zeta potential changes}

To determine the electrokinetic changes of the diluted dispersions, the zeta potential of the samples was measured. The results show that the increase of the PVA amount decreases the zeta potential in comparison to the sample without PVA (Table 3). The main reason for the zeta potential reduction can be linked to the nonionic polymer adsorption on the surface of the solid particles, which causes a decrease of the diffuse layer charge. A greater zeta potential-lowering effect can be observed between $0 \%$ and $0.25 \%$ of PVA than between $0.25 \%$ and 0.50 . At $0.50 \%$ of PVA, the surface of particles is saturated by the PVA chain; therefore, the change of zeta potential is smaller. In case of a higher concentration of PVA $(>0.50 \%)$, the steric hindrance stabilizes the system but hampers the disintegration/abrasion of the particles.

\section{Investigation of the diluted dispersions to select nanosuspension as an intermediate product}

\section{Raman investigation}

Raman spectrograms and chemical maps of raw materials and products are presented in Figures 4 and 5. The individual spectrum of $\mathrm{Mel}(\mathrm{A})$ shows that the absorption peaks are concentrated in the region from 1,600 to $1,000 \mathrm{~cm}^{-1}$ (fingerprint region), whereas the individual spectra of PVA (B and C)

Table 3 Zeta potential values of the diluted dispersions as a function of the PVA concentration and particle size distribution of Mel $( \pm S D)$

\begin{tabular}{|c|c|c|c|c|}
\hline \multirow[t]{3}{*}{ Samples } & \multicolumn{3}{|c|}{ Particle size distribution } & \multirow{3}{*}{$\begin{array}{l}\text { Zeta } \\
\text { potential } \\
(\mathrm{mV})\end{array}$} \\
\hline & $d(0.1)$ & $d(0.5)$ & $d(0.9)$ & \\
\hline & \multicolumn{3}{|c|}{ Particle size $(\mu \mathrm{m})$} & \\
\hline Mel PVA 0\% & $2.508 \pm 1.100$ & $5.762 \pm 2.700$ & $135.640 \pm 12.900$ & -30.7 \\
\hline Mel PVA $0.25 \%$ & $0.070 \pm 0.00 \mathrm{I}$ & $0.150 \pm 0.009$ & $1.478 \pm 0.0400$ & -20.9 \\
\hline Mel PVA $0.50 \%$ & $0.067 \pm 0.00 \mathrm{I}$ & $0.130 \pm 0.005$ & $0.37 I \pm 0.010$ & -16.1 \\
\hline Mel PVA $0.75 \%$ & $1.235 \pm 0.006$ & $2.611 \pm 0.018$ & $5.560 \pm 0.070$ & -15.7 \\
\hline
\end{tabular}

Abbreviations: Mel, meloxicam; PVA, poly(vinyl alcohol); $d(0.1), d(0.5), d(0.9)$, the maximum particle diameter below which $10 \%, 50 \%$, and $90 \%$ of the sample volume exists. 

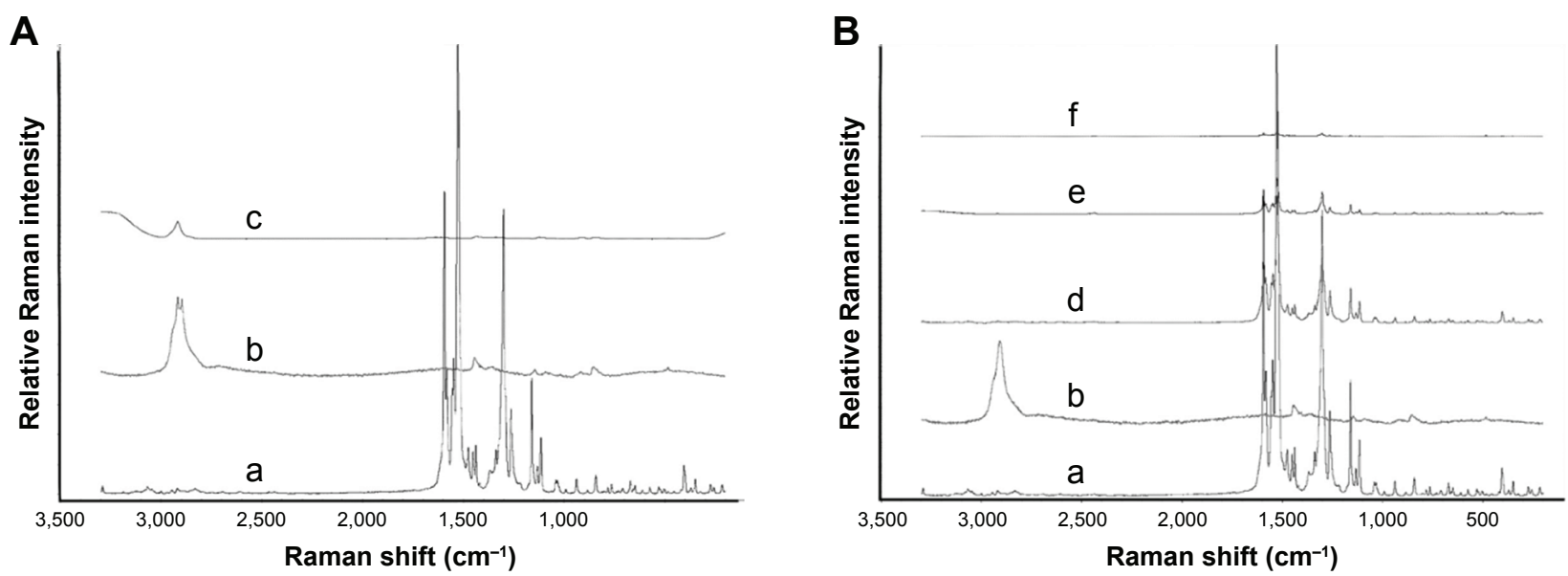

Figure 4 Investigation with Raman spectroscopy: (A) (a) spectrum of raw Mel, (b) spectrum of raw PVA, (c) spectrum of raw PVA (0.50\%) containing solution. (B) Comparison study of raw materials (Mel and PVA) and the dispersions, (d) spectrum of dispersion containing $1 \%$ Mel and $0.25 \%$ PVA, (e) spectrum of dispersion containing $1 \%$ Mel and $0.50 \%$ PVA, ( $f$ ) spectrum of dispersion containing I\% Mel and $0.75 \%$ PVA.

Abbreviations: Mel, meloxicam; PVA, poly(vinyl alcohol).

show just 1 characteristic and extended peak from 3,000 to $2,800 \mathrm{~cm}^{-1}$. This is the $\mathrm{CH}$ stretching vibration region that results a medium-to-strong intensity in Raman spectra. The spectrograms of diluted dispersions (D, E, and F) show the same characteristic region with the spectra of $\mathrm{Mel}$ - there are no detectable differences among them. Two peaks (one more characteristic in $2,437 \mathrm{~cm}^{-1}$ and another smaller one in $482 \mathrm{~cm}^{-1}$ ) appear in the spectra of PVA-containing dispersions and in spectra of aqueous Mel dispersion as well. The chemical mapping of dried dispersions profiled to this peak in 2,437 $\mathrm{cm}^{-1}$ shows that this peak cannot detected in this map. This peak can show a dissociated -OH group of Enol which is presented when Mel is in an aqueous medium, but it is disappeared in dried form, so the change is reversible. The chemical map of dispersion profiled to Mel spectrogram shows homogenous distribution of Mel. It can be summarized that there is no chemical degradation or interaction in dispersion which could be detectable with Raman technique.

\section{Morphology of particles (scanning electron microscopy)} In order to investigate the effect of milling and PVA amount on the morphology of milled Mel, the water was evaporated from dispersion and the dried samples were characterized.
A

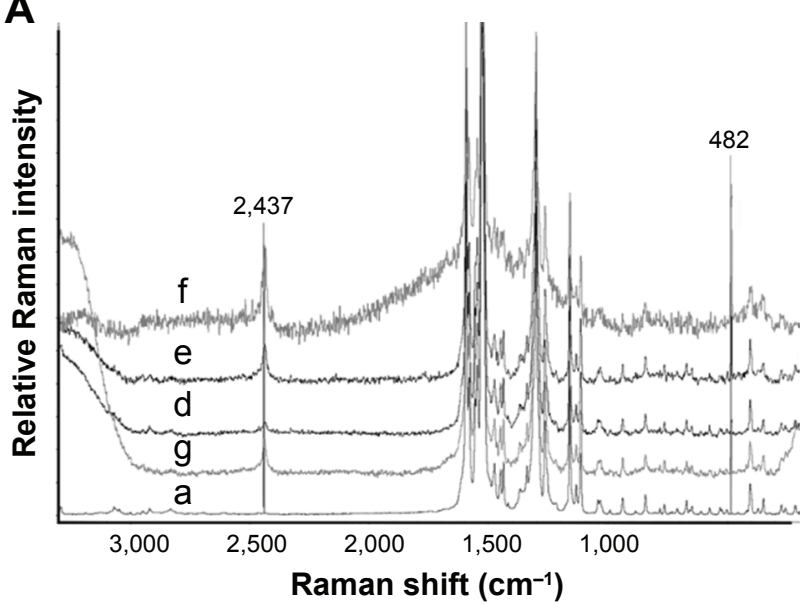

B

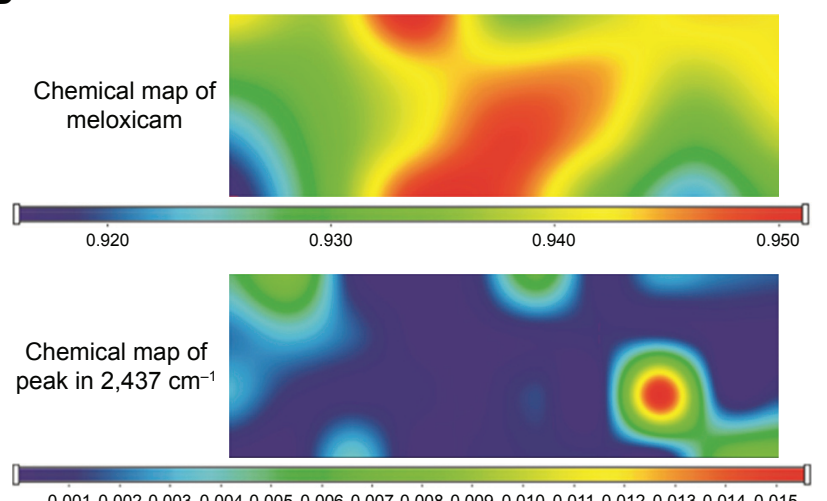

0.0010 .0020 .0030 .0040 .0050 .0060 .0070 .0080 .0090 .0100 .0110 .0120 .0130 .0140 .015

Figure 5 Investigation with Raman spectroscopy: (A) Comparing raw Mel and aqueous dispersion of Mel- and PVA-containing dispersions, (a) spectrum of raw Mel, (d) spectrum of dispersion containing I\% Mel and 0.25\% PVA, (e) spectrum of dispersion containing $1 \%$ Mel and $0.50 \%$ PVA, (f) spectrum of dispersion containing I\% Mel and $0.75 \%$ PVA, (g) spectrum of aqueous I\% Mel-containing dispersion without PVA. (B) Chemical mapping of Mel-containing dispersion (I\% Mel and 0.50\% PVA) and chemical mapping of its dried form profiled to peak in $2,437 \mathrm{~cm}^{-1}$

Abbreviations: Mel, meloxicam; PVA, poly(vinyl alcohol). 
Figure 6 shows the raw Mel in physical mixture (Mel PVA $0.50 \%$ PM) which has an irregular shape with $34.260 \pm 4.860 \mu \mathrm{m}$ as average particle size. In this PM, the PVA particles with size $<6 \mu \mathrm{m}$ are located on the surface of Mel crystals. While the aqueous dispersions had a different amount of PVA in solution form and after drying the polymer formed think film in the system, therefore the dried samples present only the Mel particles. In case of $0.25 \%$ containing PVA (Mel PVA $0.25 \%$ ), the particle size of Mel has decreased, but aggregation of the fragmented particles can be observed. Mel particles are in homogeneous disperse distribution in sample (Mel PVA $0.50 \%$ ), resulting in $<500 \mathrm{~nm}$ (average particle size: $0.130 \pm 0.005 \mu \mathrm{m}$ ). High concentration of PVA (Mel PVA 0.75\%) helped the recrystallization of Mel (nanocrystals with smooth surface) thanks to increased solubility of Mel in aqueous PVA solution. Otherwise, the sample shows heterogeneous disperse system with nano- and microparticles.

\section{Crystallinity characterization of Mel in the dried dispersions (DSC and XRPD)}

DSC was used to investigate the melting of raw Mel and raw PVA and the dried samples (Figure 7). The DSC curve of the raw Mel revealed a sharp endothermic peak at $268.66^{\circ} \mathrm{C}$, reflecting its melting point and an instantly following exothermic peak at $278.09^{\circ} \mathrm{C}$ can be observed. The DSC curve of raw PVA as a semi-crystalline polymer has 2 endothermic peaks at $169.51^{\circ} \mathrm{C}$ and at $222.74^{\circ} \mathrm{C}$. The first peak of PVA signifies a particular decrystallization of PVA and the second peak is the melting temperature. ${ }^{33}$ The DSC curves of the dried products exhibited lower melting points of Mel than that of raw Mel. It is connected to the premelting of PVA, which induces the earlier melting of Mel in proportion to the amount of PVA and decreases the crystallinity degree of Mel. Therefore, the XRPD investigation was used to check the amorphization of the active agent.

The XRPD investigations justified the change of crystallinity degree of Mel in the dispersions with different PVA amounts. Figure 8 shows the fingerprints of raw materials (Mel and PVA) and the dried dispersions. The samples show the characteristic peaks of $\mathrm{Mel}$ at $2 \theta$ values: $13.06^{\circ}, 14.94^{\circ}$, and $18.61^{\circ}$. It was found that the PVA content of the samples fundamentally influenced the decrease of the crystallinity degree of Mel. As it was established earlier in this study, low $(2.5 \%)$ and high $(7.5 \%)$ concentrations of PVA in the milled dispersion did not result in suitable milling efficiency. In this case, the crystallinity degree of Mel was $75.82 \%$ at low PVA content (2.5\%), and it decreased to $51.44 \%$ at high concentration of PVA (7.5\%). These results are connected to the milling effectiveness. In this study, the 5.0\% PVAcontaining milled dispersions showed smaller crystallinity $(13.43 \%)$ and the highest milling efficiency.

\section{Drug content determination}

The spectrophotometrically measured drug content of the samples was less than the theoretical value $(7.5 \mathrm{mg} \mathrm{Mel})$. The drug

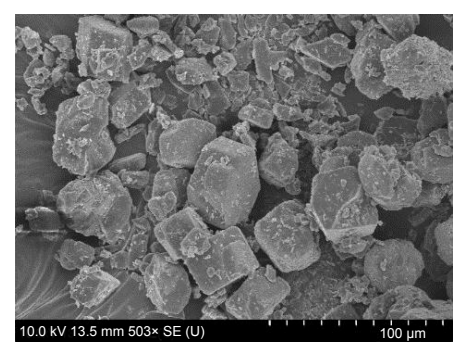

Mel PVA $0.25 \%$ PM

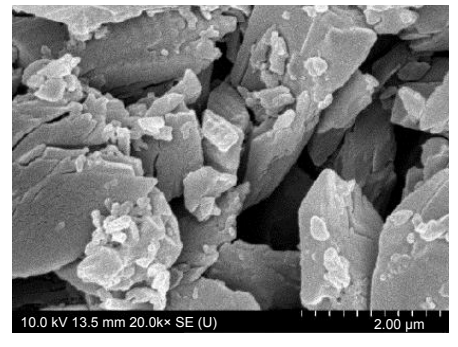

Mel PVA $\mathbf{0 . 2 5 \%}$

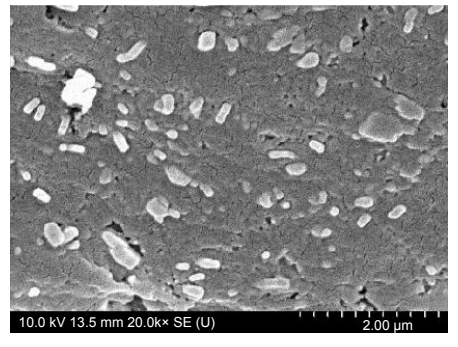

Mel PVA $0.50 \%$

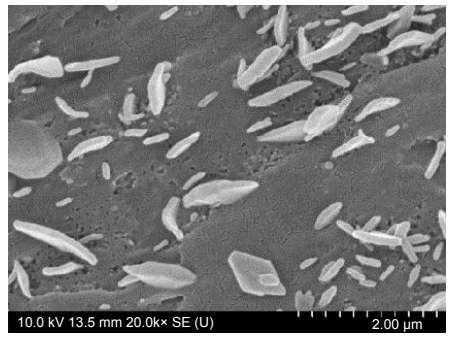

Mel PVA $0.75 \%$

Figure 6 SEM pictures of physical mixture (Mel PVA 0.25\% PM) and different PVA concentrations containing dried dispersions. Abbreviations: SEM, scanning electron microscopy; Mel, meloxicam; PVA, poly(vinyl alcohol); PM, physical mixture. 


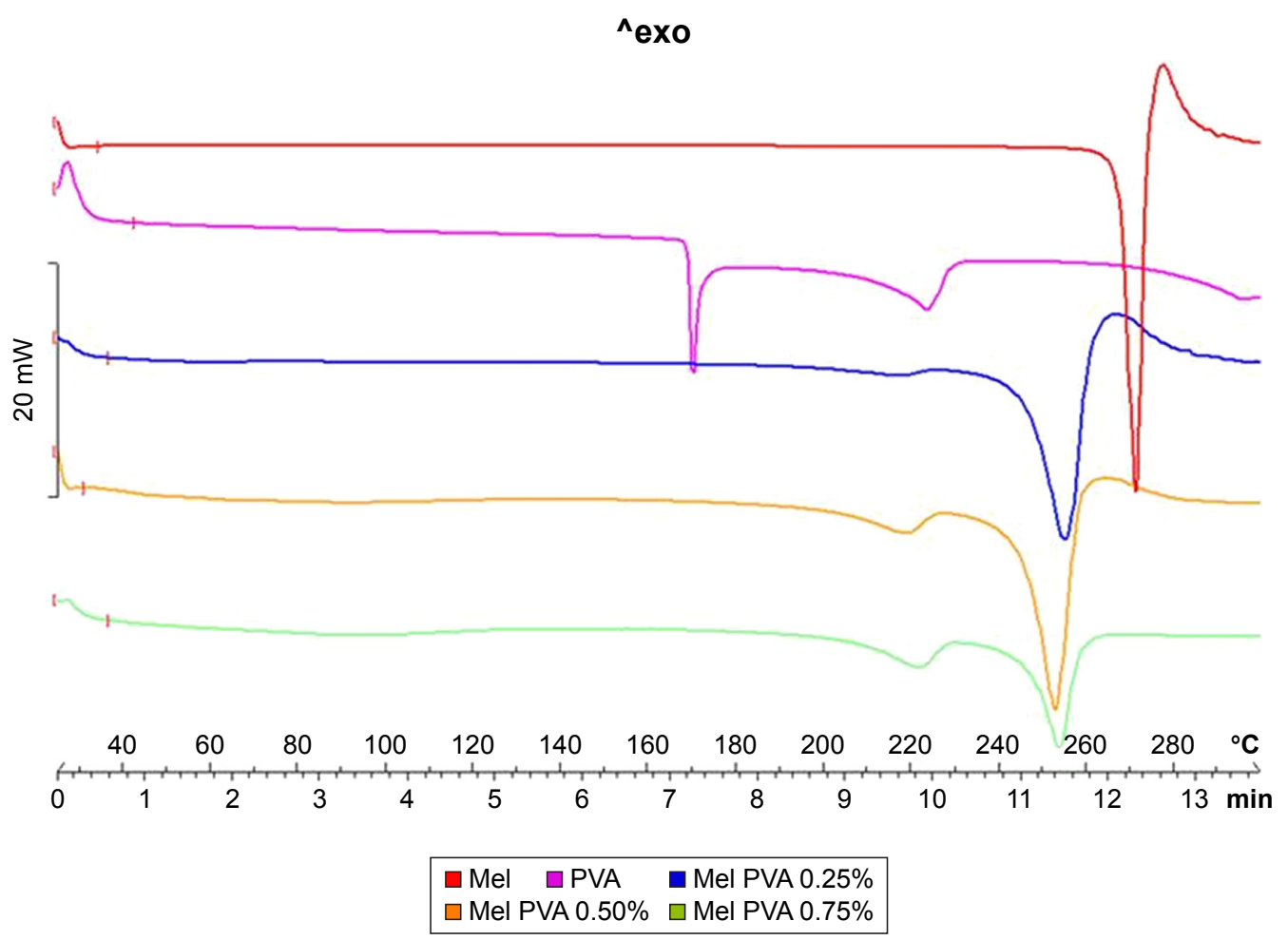

Figure 7 DSC curves of raw Mel and PVA and dried dispersions with different PVA concentrations. Abbreviations: DSC, differential scanning calorimetry; Mel, meloxicam; PVA, poly(vinyl alcohol).

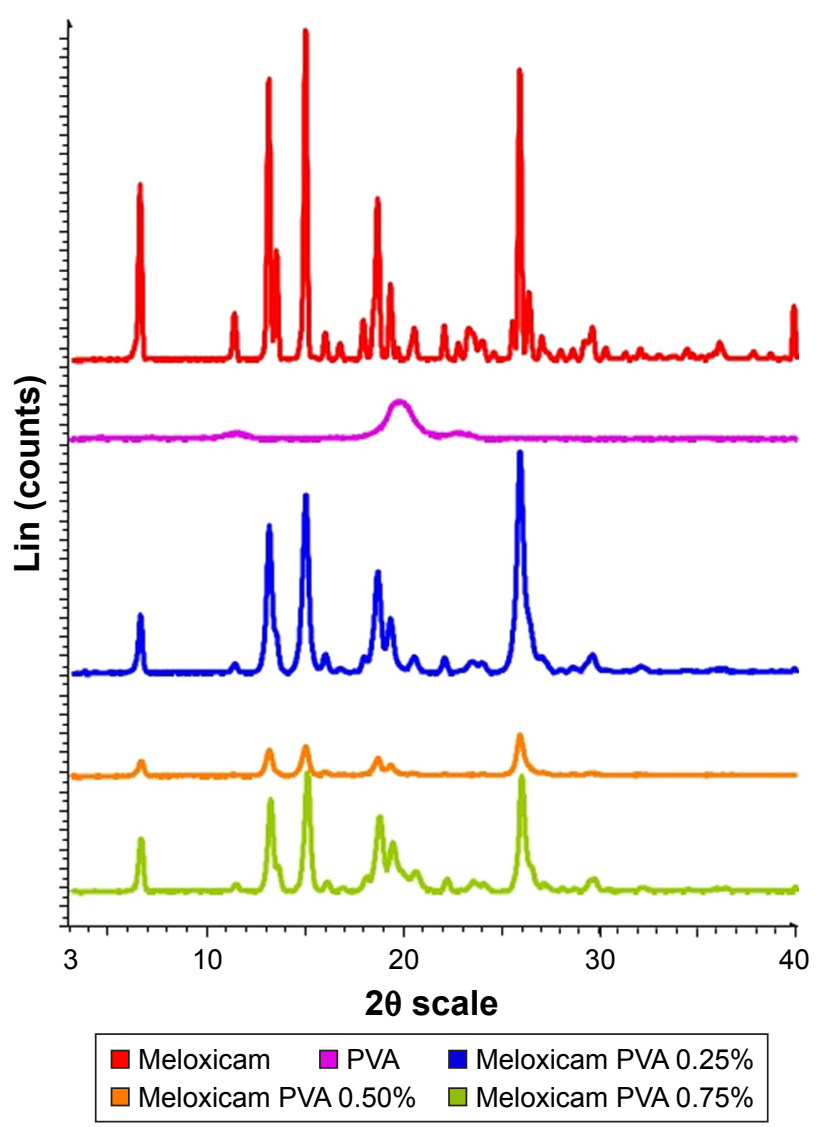

Figure 8 XRPD diffractograms of the Mel, PVA, and the dried dispersion. Abbreviations: XRPD, X-ray powder diffraction; Mel, meloxicam; PVA, poly(vinyl alcohol). contents, converted to $750 \mathrm{mg}$ dispersion quantity, are as follows: $7.36 \mathrm{mg}$ for aqueous sample (without PVA), $7.19 \mathrm{mg}$ for $0.25 \%$ PVA, $7.12 \mathrm{mg}$ for $0.50 \%$ PVA, and $7.23 \mathrm{mg}$ for $0.75 \%$. It can be stated that the washing method of the pearls resulted in greater loss of Mel in PVA-containing dispersion $(3.60 \%-5.06 \%)$ than in case of the aqueous sample without PVA (1.80\%). The amount of PVA did not significantly affect the loss of weight of Mel.

\section{In vitro dissolution study}

In case of each sample, the liberation from the hard gelatin capsules occurred within $2 \mathrm{sec}$. Mel has a weak acidic character $\left(\mathrm{p} K_{\mathrm{a}} 4.8\right)$, and therefore, its solubility in gastric juice $(\mathrm{pH}=1.2)$ is very poor $\left(1.6 \pm 0.2 \mathrm{mg} / \mathrm{L}\right.$, at $\left.37^{\circ} \mathrm{C}\right)$. In this medium, the greatest dissolved amount of $\mathrm{Mel}$ with $0.25 \%$ and $0.50 \%$ PVA content was maximum, $37 \%$, within $20 \mathrm{~min}$ (Figure 9A). This result is due to the wetting effect of PVA $(0.25 \%$ and $0.50 \%)$, which could increase the solubility of Mel, and the reduction of the particle size of Mel in the dispersions. In contrast, a higher amount of PVA $(0.75 \%)$ hinders the dissolution because a thicker polymer layer is formed on the Mel particles.

In intestinal fluid $(\mathrm{pH}=6.8)$, the dissolved amount was higher in all cases because of the better solubility of Mel $\left(0.272 \pm 0.001 \mathrm{mg} / \mathrm{mL}\right.$, at $\left.37^{\circ} \mathrm{C}\right)$. Figure $9 \mathrm{~B}$ shows that the concentration of PVA influences the amount of dissolved 

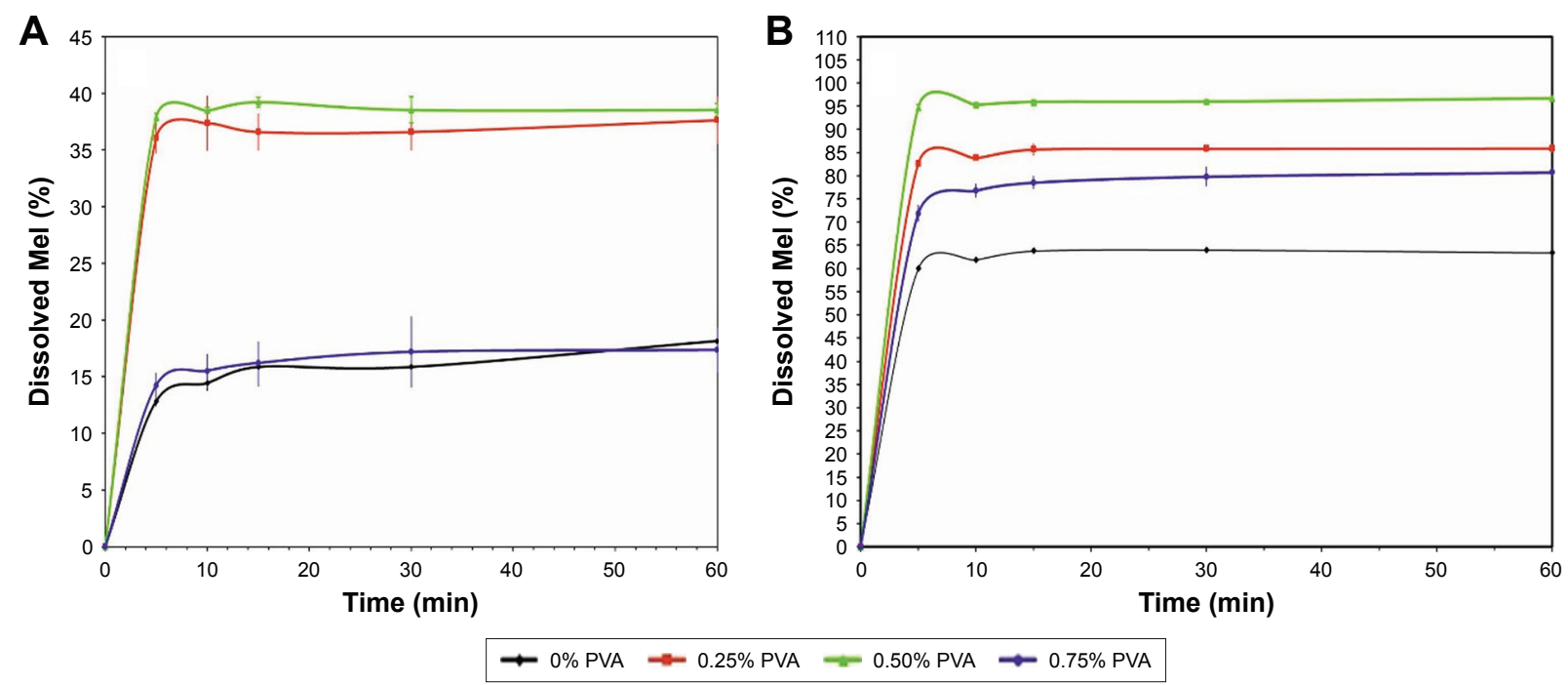

Figure 9 In vitro dissolution curves of Mel in artificial gastric juice (A) and intestinal juice (B). Abbreviations: Mel, meloxicam; PVA, poly(vinyl alcohol).

Mel as in the case of gastric juice, but its extent is bigger. In this study, the dispersion containing $0.50 \%$ of PVA had the most satisfying dissolution behavior from among the 4 samples. It is followed by the dispersions containing $0.25 \%$ and $0.75 \%$ of PVA, and finally the dispersion without PVA. The results justify the need of the polymer (PVA) and the correct choice of its quantity.

\section{Cell viability assay}

Impedance measurement, as a sensitive method to detect cellular effects, did not show significant cell damage after treatments with Mel, PVA, and Mel formulation groups, as reflected by unchanged cell index values (Figure 10). As a comparison, cells treated with the detergent Triton X-100 were lysed and a $100 \%$ toxicity was measured. The curves show the kinetics of the cellular effects of treatment solutions (Figure 10A), whereas the columns show the effect of Mel, PVA and Mel formulations at the 8-h time point (Figure 10B).

\section{Permeability study on intestinal barrier model} Caco-2 monolayers showed high TEER values $(2,660 \pm 181$ $\Omega \times \mathrm{cm}^{2}, \mathrm{n}=20$ ) before permeability experiments, indicating tight barrier properties. The average apparent permeability coefficients of marker molecules were also low (fluorescein: $0.81 \pm 0.13 \times 10^{-6} \mathrm{~cm} / \mathrm{s}$; albumin: $0.08 \pm 0.03 \times 10^{-6} \mathrm{~cm} / \mathrm{s}$ ),
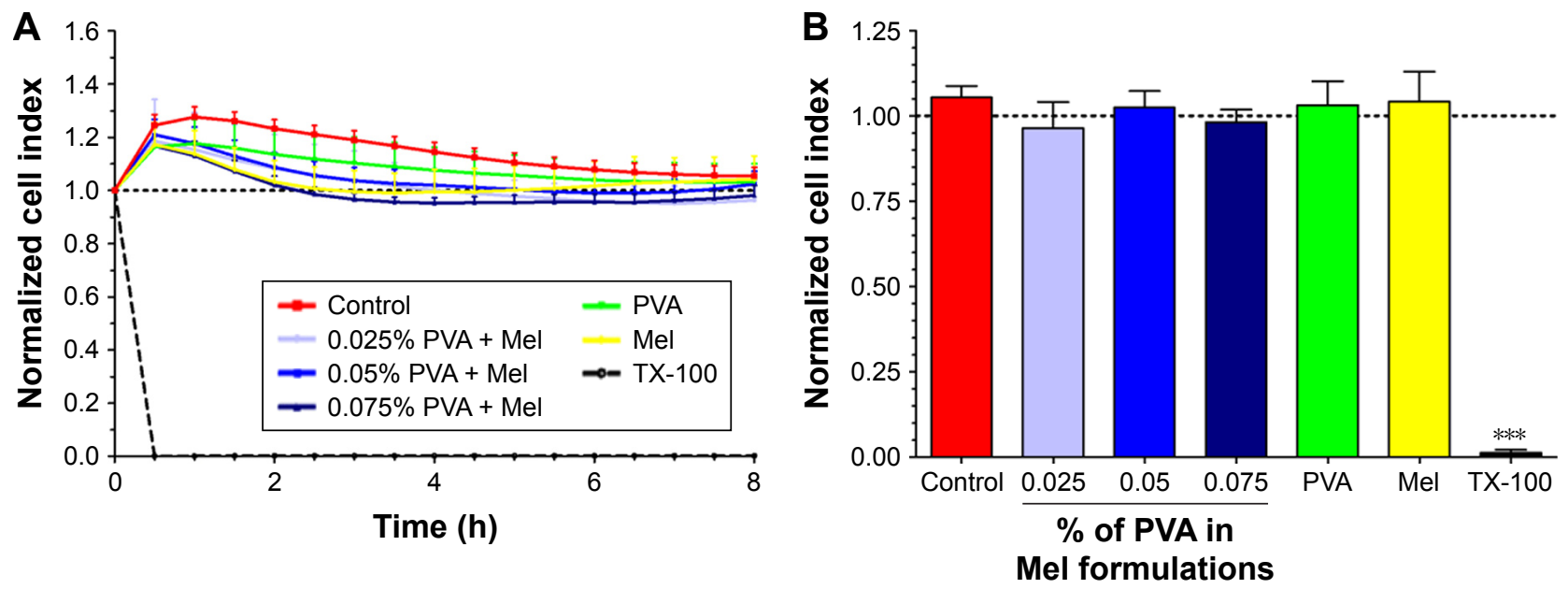

Figure 10 Cell viability kinetics (A) and results $8 \mathrm{~h}$ after treatment (B) in Caco-2 intestinal epithelial cells with Mel, PVA, and formulations measured by impedance. Notes: Values are presented as mean \pm SD, $n=6-12$. Statistical analysis: ANOVA followed by Dunett's test. Statistically significant differences are: * $p<0.05$, compared to control group.

Abbreviations: Mel, meloxicam; PVA, poly(vinyl alcohol); TX-100, Triton X-100; ANOVA, analysis of variance. 


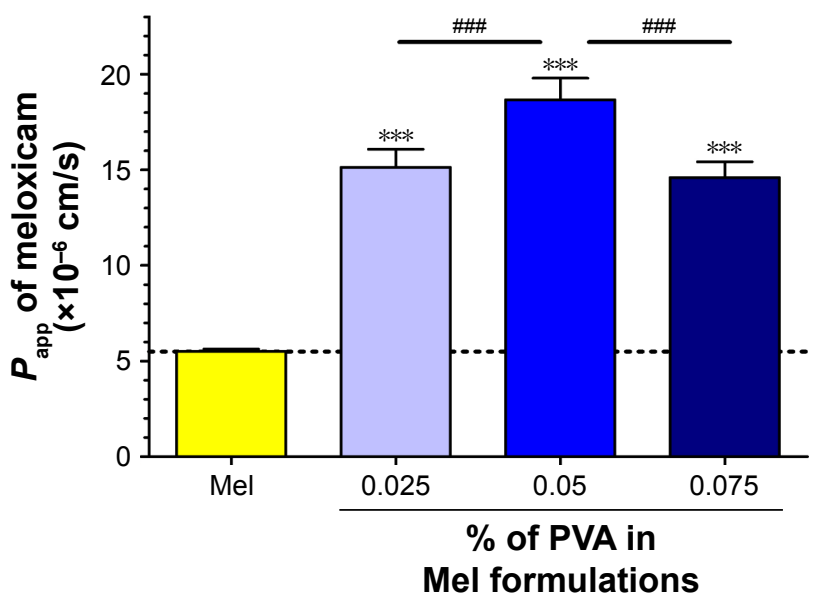

Figure II Evaluation of permeability of Mel across Caco-2 epithelial cell layers treated with Mel and optimized Mel PVA formulations for I h.

Notes: Values are presented as mean $\pm S D, n=4$. Statistical analysis: ANOVA followed by Bonferroni posttest. Statistically significant differences are: $* * * p<0.01$, compared to control group; ${ }^{\#} p<0.01$ compared to the indicated columns.

Abbreviations: Mel, meloxicam; PVA, poly(vinyl alcohol); $P_{\text {app }}$, apparent permeability coefficients.

in agreement with the TEER values and the formation of tight cell layers.

The permeability of Mel suspension and Mel formulations was significantly higher than that of marker molecules. The penetration of $\mathrm{Mel}$ from the 3 investigated products across cell layers was significantly increased as compared to Mel suspension. From all the tested samples, the $P_{\text {app }}$ value of Mel was the highest in the formulation containing $0.05 \%$ PVA (Figure 11).

\section{Holding time determination}

Since the investigated dispersions are intermediate products, the change of particle size distribution and the crystallinity index are very important during storage. In general, the time period before the dispersion used for the preparation of different dosage forms is 1 or $2 \mathrm{~h}$, or possibly longer. The measurements have proven that $0.50 \%$ of PVA-containing dispersion had no significant changes in the particle size, particle size distribution, and crystallinity up to 2 weeks (Table 4).

Table 4 Particle size distribution changes $(\mu \mathrm{m})$ during the stability testing

\begin{tabular}{|c|c|c|c|c|c|c|}
\hline & \multicolumn{3}{|l|}{ Day 0} & \multicolumn{3}{|c|}{2 weeks } \\
\hline & $d(0.1)$ & $d(0.5)$ & $d(0.9)$ & $d(0.1)$ & $d(0.5)$ & $d(0.9)$ \\
\hline Mel PVA 0.25\% & 0.070 & 0.150 & $\mathrm{I} .478$ & 0.080 & 0.152 & 2.073 \\
\hline Mel PVA $0.50 \%$ & 0.067 & 0.136 & 0.371 & 0.068 & 0.140 & 0.427 \\
\hline Mel PVA $0.75 \%$ & 1.207 & 2.232 & 5.224 & 0.244 & 2.611 & 5.560 \\
\hline
\end{tabular}

Abbreviations: Mel, meloxicam; PVA, poly(vinyl alcohol); $d(0.1), d(0.5), d(0.9)$, the maximum particle diameter below which $10 \%, 50 \%$, and $90 \%$ of the sample volume exists.

\section{Conclusion}

Based on our results, it can be stated that the combination of planetary ball mill and the pearl milling technology is a new possibility to nanonize the Mel as a nonsteroidal anti-inflammatory agent to reach the particle size range of 100-500 nm. This combinative wet technology resulted in significant particle size reduction without premilling (pretreatment of raw agent). In addition to the process parameters (the pearl amount, the milling time, and the rotation speed of jar), the amount of PVA was also a critical parameter because it affected the milling effectiveness, the particle size distribution, and the crystallinity of Mel. The different concentrations of PVA in the aqueous dispersion also influenced the viscosity and the electrokinetic property of the particle, according the DLVO theory, ${ }^{34}$ and thus, the stability of the dispersions.

It was found that the milling effectiveness of low concentration of PVA $(<4 \%)$ was not satisfactory, because the crushing/breaking effect of the pearls could less prevail. High concentration of PVA $(>5 \%)$ also resulted in unsatisfactory milling effectiveness because of the formation of polymer layer on surface of particles, which protects the particles from the fragmentation. In connection with this, the crystallinity of Mel decreased with the increase of milling effectiveness, which plays an important role in the fast drug release.

In this work, the combined wet milling process was also used successfully to prepare Mel-containing nanosuspension as an intermediate product to design the final dosage form(s) for per oral administration. The optimized process parameters (1:1 ratio of predispersion and pearls, $437 \mathrm{rpm}$, and $43 \mathrm{~min}$ ) resulted in 200 -fold particle size reduction of Mel. Considering the effectiveness of milling, 5\% PVA was proved to be an optimal quantity to meet the expected value $(100-500 \mathrm{~nm})$. The optimized nanosuspension $(1 \%$ Mel and $0.50 \%$ PVA) as an intermediate product showed a stable system with 2 weeks of holding time.

The human Caco-2 cell culture studies justified that the penetration of Mel from different PVA-containing products was significantly increased as compared to Mel suspension without toxic effects. From all the tested samples, the $P_{\text {app }}$ value of Mel was the highest in the investigated sample containing $0.05 \%$ PVA, which belongs to the optimized nanosuspension.

Based on the above results, the milling process and the composition of the nanosuspension can be recommended to produce innovative dosage forms (eg, capsule) with fast dissolution rate of $\mathrm{Mel}$. During the development of dosage form(s), the stabilization of the amorphous Mel particle may 
also be an important viewpoint to ensure the dissolution profile of the active ingredient.

\section{Author contribution}

It is connected.

\section{Acknowledgment}

This work was supported by Gedeon Richter Ltd - GINOP project (2.2.1-15-2016-00007).

\section{Disclosure}

The authors report no conflicts of interest in this work.

\section{References}

1. Andric L, Terzic A, Timovic-Pavlovic Z, Trumic M, Petrov M, Pavlovic L. A kinetic study of micronization grinding of dry mica in a planetary ball mill. Adv Mater Sci Eng. 2013;2013:543857.

2. Szafraniec J, Antosika A, Knapik-Kowalczuk J, et al. Planetary ball milling and supercritical fluid technology as a way to enhance dissolution of bicalutamide. Int J Pharm. 2017;533(2):470-479.

3. Liu P, Rong X, Laru J, et al. Nanosuspensions of poorly soluble drugs: preparation and development by wet milling. Int J Pharm. 2011; 411(1-2):215-222.

4. Ghosh I, Schenck D, Bose S, Ruegger C. Optimization of formulation and process parameters for the production of nanosuspension by wet media milling technique: effect of vitamin E TPGS and nanocrystal particle size on oral absorption. Eur J Pharm Sci. 2012;47(4):718-728.

5. George M, Ghosh I. Identifying the correlation between drug/stabilizer properties and critical quality attributes (CQAs) of nanosuspension formulation prepared by wet media milling technology. Eur J Pharm Sci. 2013;48(1-2):142-152.

6. Sushant S, Archana K. Methods of size reduction and factors affecting size reduction in pharmaceutics. Int Res J Pharm. 2013;4(8):57-64.

7. Bhakay A, Merwade M, Bilgili E, Dave RN. Novel aspects of wet milling for the production of microsuspensions and nanosuspensions of poorly water-soluble drugs. Drug Dev Ind Pharm. 2011;37(8):963-976.

8. Rasenack N, Müller BW. Micron-size drug particles: common and novel micronization techniques. Pharm Dev Technol. 2004;9(1):1-13.

9. Bilgili E, Hamey R, Scarlett B. Nano-milling of pigment agglomerates using a wet stirred media mill: elucidation of the kinetics and breakage mechanisms. Chem Eng Sci. 2006;61(1):149-157.

10. Loh ZH, Samanta AK, Heng PWS. Overview of milling techniques for improving the solubility of poorly water-soluble drugs. Asian J Pharm Sci. 2015;10(4):255-274.

11. Pawar VK, Singh Y, Meher JG, Gupta S, Chourasia MK. Engineered nanocrystal technology: in-vivo fate, targeting and applications in drug delivery. J Control Release. 2014;183:51-66.

12. Azad AM, Afolabi A, Patel N, Dave R, Bilgili E. Preparation of stable colloidal suspensions of superdisintegrants via wet stirred media milling. Particuology. 2014;14:76-82.

13. Patravale VB, Date AA, Kulkarni RM. Nanosuspensions: a promising drug delivery strategy. J Pharm Pharmacol. 2004;56(7):827-840.

14. Peltonen L, Hirvonen J. Pharmaceutical nanocrystals by nanomilling: critical process parameters, particle fracturing and stabilization methods. J Pharm Pharmacol. 2010;62(11):1569-1579.

15. Chen H, Khemtong C, Yang X, Chang X, Gao J. Nanonization strategies for poorly water-soluble drugs. Drug Discov Today. 2011;16(7-8): 354-360.

16. Johansson A. Correlation Between Process Parameters and Milling Efficiency [dissertation]. Uppsala: Uppsala University; 2012.
17. Web reference 6 Retsch $^{\circledR}$. Available from: http://pdf.directindustry. com/pdf/retsch/the-sample-high-energy-ballmills/19308-518973.html. Accessed on March 20, 2016.

18. Van Eerdenbrugh B, Froyen L, Van Humbeeck J, Martensc JA, Augustijnsa P, Van Den Mootera G. Alternative matrix formers for nanosuspension solidification: dissolution performance and X-ray microanalysis as an evaluation tool for powder dispersion. Eur JPharm Sci. 2008;35(4):344-353.

19. VanEerdenbrugh B, Froyen L, Van Humbeeck J, Martens JA, Augustijns P, Van den Mooter G. Drying of crystalline drug nanosuspensions - the importance of surface hydrophobicity on dissolution behavior upon redispersion. Eur J Pharm Sci. 2008;35(1-2):127-135.

20. Mártha C, Kürti L, Farkas G, et al. Effects of polymers on the crystallinity of nanonized meloxicam during a co-grinding process. Eur Polym J. 2013;49(9):2426-2432.

21. Bartos C, Ambrus R, Sipos P, et al. Study of sodium hyaluronate-based intranasal formulations containing micro- or nanosized meloxicam particles. Int J Pharm. 2015;491(1-2):198-207.

22. Gieszinger P, Csóka I, Pallagi E, et al. Preliminary study of nanonized lamotrigine containing products for nasal powder formulation. Drug Des Devel Ther. 2017;11:2453-2466.

23. Bartos C, Szabó-Révész P, Bartos C, Katona G, Jójárt-Laczkovich O, Ambrus R. The effect of an optimized wet milling technology on the crystallinity, morphology and dissolution properties of micro- and nanonized meloxicam. Molecules. 2016;21(4):507-518.

24. Chibowski S, Paszkiewicz M, Krupa M. Investigation of the influence of the polyvinyl alcohol adsorption on the electrical properties of $\mathrm{Al}_{2} \mathrm{O}_{3}$ solution interface, thickness of the adsorption layers of PVA. Powder Technol. 2000;107(3):251-255.

25. Vastag M, Keseru GM. Current in vitro and in silico models of bloodbrain barrier penetration: a practical view. Curr Opin Drug Discov Devel. 2009;12(1):115-124.

26. Hellinger E, Bakk ML, Pocza P, Tihanyi K, Vastag M. Drug penetration model of vinblastine-treated Caco-2 cultures. Eur J Pharm Sci. 2010; 41(1):96-106.

27. Li Q, Wang K, Zheng Y, Ruan M, Mei X, Lin B. Experimental research of particle size and size dispersity on the explosibility characteristics of coal dust. Powder Technol. 2016;292:290-297.

28. Bocsik A, Walter FR, Gyebrovszki A, et al. Reversible opening of intercellular junctions of intestinal epithelial and brain endothelial cells with tight junction modulator peptides. J Pharm Sci. 2016;105(2): 754-765.

29. Kiss L, Walter FR, Bocsik A, et al. Kinetic analysis of the toxicity of pharmaceutical excipients Cremophor EL and RH40 on endothelial and epithelial cells. J Pharm Sci. 2013;102(4):1173-1181.

30. Kürti L, Veszelka S, Bocsik A, et al. The effect of sucrose esters on a culture model of the nasal barrier. Toxicol In Vitro. 2012;26(3): 445-454.

31. Hellinger E, Veszelka S, Tóth AE, et al. Comparison of brain capillary endothelial cell-based and epithelial (MDCK-MDR1, Caco-2, and VBCaco-2) cell-based surrogate blood-brain barrier penetration models. Eur J Pharm Biopharm. 2012;82(2):340-351.

32. Kiss L, Hellinger É, Pilbat AM, et al. Sucrose esters increase drug penetration, but do not inhibit p-glycoprotein in caco-2 intestinal epithelial cells. J Pharm Sci. 2014;103(10):3107-3119.

33. Tretinnikov ON, Zagorskaya SA. Determination of the degree of crystallinity of poly(vinyl alcohol) by FTIR spectroscopy. $J$ Appl Spectrosc. 2012;79(4):521-526.

34. Derjaguin B, Landau L. Theory of the stability of strongly charged lyophobic sols and of the adhesion of strongly charged particles in solutions of electrolytes. Acta Physicochim URSS. 1941;14:633-662. 


\section{Publish your work in this journal}

Drug Design, Development and Therapy is an international, peerreviewed open-access journal that spans the spectrum of drug design and development through to clinical applications. Clinical outcomes, patient safety, and programs for the development and effective, safe, and sustained use of medicines are the features of the journal, which

has also been accepted for indexing on PubMed Central. The manuscript management system is completely online and includes a very quick and fair peer-review system, which is all easy to use. Visit http://www.dovepress.com/testimonials.php to read real quotes from published authors.

Submit your manuscript here: http://www.dovepress.com/drug-design-development-and-therapy-journal 


\section{PUBLICATION III.}




\title{
Robustness testing of milling process, analyzing the particle size distribution and crystallinity of the milled samples
}

\author{
Csaba Bartos $^{1}$ • Orsolya Jójárt-Laczkovich ${ }^{1}$ - Géza Regdon Jr. ${ }^{1} \cdot$ Piroska Szabó-Révész $^{1}$ (D)
}

Received: 12 December 2018/Accepted: 15 May 2019

(c) The Author(s) 2019

\begin{abstract}
The aim of this work was to discover the robustness of combined wet milling process to determine the interval of the Mel (meloxicam) amount and to predict the degree of crystallinity of the milled samples using DSC and XRPD measurements. The samples had the poly(vinyl alcohol) (PVA) as the stabilizing agent. By increasing the amount of the Mel, its crystallinity increased and close correlation was found between the degree of crystallinity and the Mel amount. To achieve the desired particle size range (100-500 nm), the Mel amount should be changed between 10.0 and 17.5\% (w/w) and a PVA concentration should be used between 5.0 and $4.58 \%(\mathrm{w} / \mathrm{w})$. In this specified range, the degree of crystallinity of Mel will be changed between 20 and 45\%. The crystallinity of Mel investigated by DSC and XRPD did not show any significant difference at $95 \%$ significance level. During the milling process to predict the amorphous content of the active agent, the DSC method can be suggested for the fast quantification of the degree of crystallinity.
\end{abstract}

Keywords Crystallinity $\cdot$ Meloxicam · Nanoparticles $\cdot$ Poly(vinyl alcohol) $\cdot$ Wet milling

\section{Introduction}

Dry and wet milling technologies as disintegration methods are conventionally and commonly used in the pharmaceutical industry $[1,2]$. Drugs which belong to Class II of BCS (Biopharmaceutical Classification System) have low water solubility and high permeability; therefore, their bioavailability can increase with the decrease in particle size to the nanosize range, resulting in a high specific surface and a fast dissolution rate. During milling, amorphization of active agent can occur due to the high energy impact [3, 4]. Amorphization is also a well-known possibility to enhance the dissolution rate of drugs with low water solubility. Due to the lack of crystalline structure and the crystal lattice breaking force, less energy is needed for solubilization. Nevertheless, additional stabilization is needed for the preservation of the amorphous form from recrystallization [5-7].

Piroska Szabó-Révész

revesz@pharm.u-szeged.hu

1 Institute of Pharmaceutical Technology and Regulatory Affairs, University of Szeged, Eötvös u 6, Szeged 6720, Hungary
Our main research topic is the nanonization of different active agents using a wet milling process, where the planetary ball mill is combined with pearl milling technology [8, 9]. In our previous research to reach a nanoparticle size range $(100-500 \mathrm{~nm})$, the process parameters (milling time, rotation speed, amount of pearl and the ratio of drug to excipient) were optimized. The samples were produced with the addition of the aqueous solution of poly(vinyl alcohol) (PVA) as the stabilizing agent. The main purpose of this work was to increase the dissolution rate and extent of poorly water-soluble meloxicam (Mel) as a nonsteroidal anti-inflammatory drug. In this system, the Mel amount was $10 \%(w / w)$ and $5 \%(w /$ w) of PVA proved to be an optimal quantity to meet the expected particle size range. During the milling, a high level of amorphization was detected with DSC and XRPD examinations [10]. In this milling system, the ratio of the started pre-suspension and pearls 1:1 (w/w) resulted in about 200-fold particle size reduction in one step. In connection with this result, questions arose: (1) whether the amount of the active ingredient has a milling effect and (2) whether the particle size distribution and the degree of crystallinity can be designed by varying the amount of the 
active ingredient. These questions are important for the robustness of the process and the extent of scale-up [11].

Based on the above, the aim of the present work was to discover the robustness of our optimized combined wet milling process to determine the interval of the Mel amount which can be successfully nanonized (100-500 nm) and in this connection to predict the crystallinity degree of the milled samples using DSC as a fast analyzing method and to verify it with XRPD measurements.

\section{Experimental}

\section{Materials}

Meloxicam (raw Mel) was obtained from EGIS Ltd. (Budapest, Hungary). PVA-Mowiol 4-98 (Mw 27,000) (Sigma-Aldrich Co. LLC, St Louis, MO, USA) was used as a stabilizing agent. Zirconium oxide $\left(\mathrm{ZrO}_{2}\right)$ beds with a diameter of $0.3 \mathrm{~mm}$ were obtained from Netzsch (Netzsch $\mathrm{GmbH}$, Selb, Germany). All reagents were purchased from Sigma-Aldrich Ltd. (Budapest, Hungary).

\section{Design of the sample series}

Eight samples were prepared from 2.5 to $20.0 \%$ of Mel with $2.5 \%$ increment per sample. As the stabilizing agent, $5.0 \%$ of PVA aqueous solution as initial concentration was added up to $20.0 \mathrm{~g}$ to each sample, which was selected on the basis of our previous experiments [10]. Thus, the PVA concentration with $0.14 \%$ increment per sample was between 4.44 and $5.42 \%$. The Mel and PVA concentrations of the samples are shown in Table 1. For the DSC and XRPD investigations, the samples were desiccated in a vacuum desiccator at $40{ }^{\circ} \mathrm{C}$ to constant mass.

Table $1 \mathrm{Mel}$ and PVA concentrations in the samples

\begin{tabular}{lcl}
\hline Sample name & Mel\%/w/w & PVA\%/w/w \\
\hline Mel_0.5 g & 2.50 & 5.42 \\
Mel_1.0 g & 5.00 & 5.28 \\
Mel_1.5 g & 7.50 & 5.14 \\
Mel_2.0g & 10.00 & 5.00 \\
Mel_2.5 g & 12.50 & 4.86 \\
Mel_3.0 g & 15.00 & 4.72 \\
Mel_3.5 g & 17.50 & 4.58 \\
Mel_4.0 g & 20.00 & 4.44 \\
\hline
\end{tabular}

${ }^{\mathrm{a} O p t i m i z e d ~ s a m p l e ~}$

\section{Combined wet media milling}

The samples were ball-milled in a 50-mL steel jar (Retsch PM 100 MA, Retsch GmbH, Haan, Germany) with 0.3-mm $\mathrm{ZrO}_{2}$ beads as the grinding media. The process parameters optimized in our pervious work were as follows: $437 \mathrm{rpm}$, $43 \mathrm{~min}$ and $20 \mathrm{~g}$ of milling media which was used for every process (dispersion to grinding media ratio 1:1) [10]. The temperature of the samples, measured immediately after milling, did not exceed $39^{\circ} \mathrm{C}$.

\section{Particle size measurement}

The volume-based particle size distribution was measured by laser diffraction (Mastersizer S 2000, Malvern Instruments Ltd., Worcestershire, UK). The refractive index for Mel was 1.596. Water was used as a dispersant with a refractive index of 1.330. In all cases, the particle size distributions were characterized by $d(0.1), d(0.5)$ and $d(0.9)$ (where, for example, $d(0.5)$ is the maximum particle diameter below which $50 \%$ of the sample volume exists). Span values were calculated according to Eq. 1. A high span value $(>1)$ denotes a broad particle size distribution [12].

Span $=\frac{d(0.9)-d(0.1)}{d(0.5)}$.

\section{Differential scanning calorimetry}

Differential scanning calorimetry (DSC) was performed with a Mettler-Toledo DSC 821e (Mettler-Toledo GmbH, Switzerland) instrument. DSC curves were evaluated with STARe Software. The starting and final temperatures were $25{ }^{\circ} \mathrm{C}$ and $300{ }^{\circ} \mathrm{C}$ with $10{ }^{\circ} \mathrm{C} \min ^{-1}$ heating rate. Argon atmosphere of $100 \mathrm{~mL} \mathrm{~min}{ }^{-1}$ was used in all cases as an inert gas. Eight physical mixtures equal to the treated samples were examined as a reference scale. The calculations of Mel crystallinity (Cryst\%) were performed using the total area under the curve (AUC) of the melting enthalpy of the milled samples (AUCMelM) and the physical mixtures (AUCMelPM). The values were compared using Eq. 2:

Cryst $\%=\frac{\text { AUCMelM }}{\text { AUCMelPM }} * 100$.

\section{Thermogravimetric analysis-water content determination}

To monitor the water content of the sample after desiccation, TG measurements were performed. The water content determination was conducted based on the mass loss of the sample during heating in the interval of $25-120{ }^{\circ} \mathrm{C}$. 
Thermogravimetric analysis was carried out with a Mettler-Toledo TGA/DSC1 (Mettler-Toledo GmbH, Switzerland) instrument. Curves were evaluated with STARe Software. The starting and final temperatures were $25{ }^{\circ} \mathrm{C}$ and $300{ }^{\circ} \mathrm{C}$ with $10{ }^{\circ} \mathrm{C} \mathrm{min}^{-1}$ heating rate. Sample mass varied between 10 and $12 \mathrm{mg}$.

\section{X-ray powder diffractometry (XRPD)}

The X-ray powder diffraction patterns (XRPDs) were obtained with Bruker D8 Advance (Bruker AXS, Germany) equipped with a Sycos H-Hot (Ansyco GmbH, Karlsruhe, Germany) programmable plate holder. Results were detected with a Våntec-1 detector. The patterns were recorded at a tube voltage of $40 \mathrm{kV}$ and tube current of $40 \mathrm{~mA}$, applying a step size of $0.01 \AA 2 \mathrm{~h}$ in the angular range of 3-40 $2 \mathrm{~h}$. Eight physical mixtures equal to the treated samples were examined as a reference scale. The determination of Mel crystallinity (Cryst\%) was also performed using the total area under the curve (AUC) of three characteristic peaks $\left(13.06^{\circ}, 14.94^{\circ}\right.$ and $\left.18.61^{\circ} 2 \Theta\right)$ of the milled samples and the physical mixtures. The calculation was the same as in the case of DSC measurements.

\section{Results and discussion}

\section{Particle size measurements}

Using the optimized process parameters, based on the change in the amount of Mel, the particle size distribution is one of the critical parameters. In this respect, the comparison is based on the particle size of the optimized sample "Mel_2.0 g", which had 10\% of MEL and 5\% of PVA (Table 2).

After milling, the different Mel amounts containing nanosuspensions showed same $d(0.5)$ value, which means

Table 2 Results of the particle size measurements

\begin{tabular}{llrr}
\hline Sample name & $d(0.5) / \mu \mathrm{m}$ & \multicolumn{1}{c}{$d(0.9) / \mu \mathrm{m}$} & \multicolumn{1}{c}{ Span } \\
\hline Mel_raw & $34.26 \pm 4.86$ & $73.59 \pm 27.11$ & 1.815 \\
Mel_0.5 g & $0.138 \pm 0.027$ & $1.273 \pm 0.134$ & 8.662 \\
Mel_1.0 g & $0.142 \pm 0.023$ & $1.949 \pm 0.117$ & 10.207 \\
Mel_1.5 g & $0.141 \pm 0.018$ & $0.87 \pm 0.076$ & 5.676 \\
Mel_2.0g & $0.136 \pm 0.003$ & $0.371 \pm 0.043$ & 2.240 \\
Mel_2.5 g & $0.139 \pm 0.006$ & $0.413 \pm 0.037$ & 2.655 \\
Mel_3.0 g & $0.145 \pm 0.008$ & $0.436 \pm 0.045$ & 2.956 \\
Mel_3.5 g & $0.141 \pm 0.013$ & $0.448 \pm 0.050$ & 2.700 \\
Mel_4.0 g & $0.140 \pm 0.021$ & $0.974 \pm 0.072$ & 6.448 \\
\hline
\end{tabular}

${ }^{\mathrm{a} O p t i m i z e d ~ s a m p l e}$ that the maximum particle diameter of $50 \%$ of the sample volume is less than $150 \mathrm{~nm}$. By contrast, the $d(0.9)$ values, in the case of smaller and higher amounts of Mel, were already outside the upper range of the desired value $(500 \mathrm{~nm})$. It can be assumed that the smaller amount of Mel $(<10 \%)$ decreased the number of successful collisions of Mel and the milling media. The larger amount of Mel $(>17.5 \%)$, however, increased the density of the sample and reduced milling efficiency. Span values, which contain the $d(0.1)$ values, confirm the suitability of particle size distribution in the range of 10\% (Mel_2.0 g) and 17.5\% of Mel (Mel_3.5 g).

\section{TG measurements}

PVA is a hygroscopic agent; therefore, its residual water content was controlled. This is a semicrystalline polymer with a well-defined glass transition temperature at $45-50{ }^{\circ} \mathrm{C}$ in the case of less than $1.5 \%$ water content. Higher water content of PVA can drastically decrease the glass transition temperature (up to $20^{\circ} \mathrm{C}$ ), which can influence the thermal behavior of PVA-containing compositions [13]. Mel has a hydrophobic character; its water content is less than $0.01 \%$. TG curves between 25 and $160{ }^{\circ} \mathrm{C}$ showed fast and then slow mass loss, which is connected to the PVA amount. The samples with smaller Mel and higher PVA concentration showed more water content. It can be stated that the polymer amount has a significant role in the residual water content of the samples and, consequently, the glass transition temperature (Fig. 1).

\section{DSC analysis}

For the crystallinity characterization of Mel, first raw Mel and PVA were investigated (Fig. 2). The DSC curve of raw Mel has a sharp characteristic melting peak at $268.66{ }^{\circ} \mathrm{C}$ (onset 266.83 , endset $273.27^{\circ} \mathrm{C}$ ), and an instantly following exothermic peak can be observed at $279.09{ }^{\circ} \mathrm{C}$. PVA has two endothermic peaks at $169.51{ }^{\circ} \mathrm{C}$ and

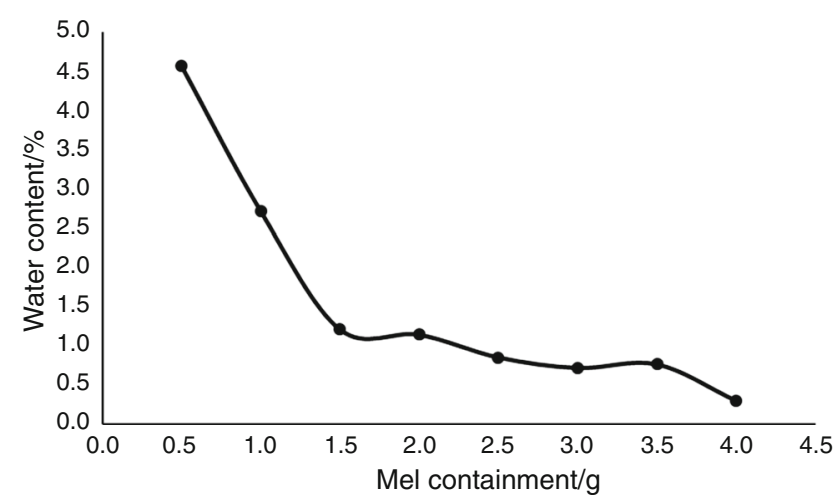

Fig. 1 Water content of the milled samples 
Fig. 2 DSC curves of raw Mel, PVA and different Melcontaining milled samples $\wedge^{\wedge}$ Exo

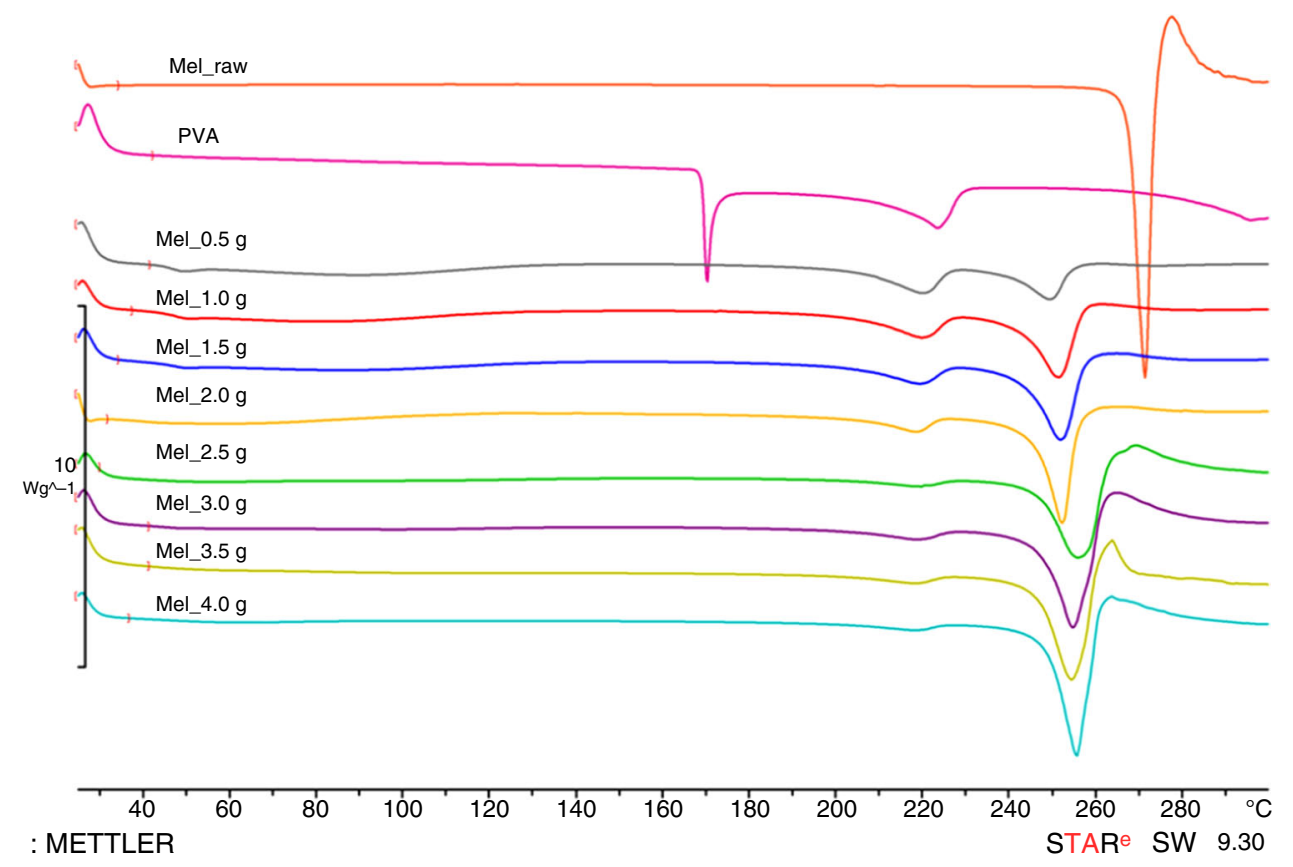

222.74 ${ }^{\circ} \mathrm{C}$. Since PVA is a mixture of crystalline and amorphous fractions, it is assumed that the first endothermic peak shows a structural change in part of the crystalline fraction and the second one presents the melting point of PVA. It should be noted that PVA has deacetylation in the temperature range $160-400{ }^{\circ} \mathrm{C}$ and the total degradation corresponds to the degradation of vinyl acetate and vinylpyrrolidone at $396{ }^{\circ} \mathrm{C}$ and $484{ }^{\circ} \mathrm{C}$, respectively [14].

The DSC curves of the samples exhibit that the increase in the Mel amount $(>10.0 \%)$ results in a sharper endothermic peak, approaching the melting point of raw Mel. In accordance with this, the signs of PVA are weakened, which can be associated with its reduced amount $(<5.0 \%)$. By decreasing the Mel amount $(<10.0 \%)$, the endothermic peaks will be less sharp and the signs of PVA are strengthened. It is connected to the higher amount of PVA $(>5.0 \%)$. The areas of the melting enthalpies already predict the change in the degree of crystallinity of Mel.

In order to determine the degree of crystallinity of Mel, taking into account the influence of PVA, the physical mixtures of the milled samples formed the basis.

Figure 3 presents the degree of crystallinity measured by DSC as a function of the Mel amount in the sample. By increasing the Mel amount, the crystallinity of the milled products was increased. A close correlation $\left(R^{2}=0.9587\right)$ was found between crystallinity and the Mel amount. It can be seen that increasing the amount of Mel $(>10 \%)$ significantly increased the degree of crystallinity of the milled

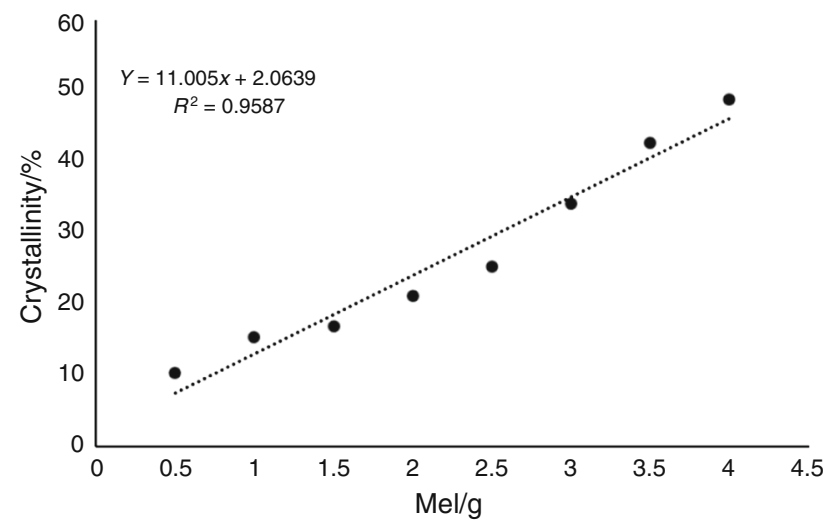

Fig. 3 Relation between the crystallinity of Mel and its amount in the milled samples measured by XRPD

sample (from 21.21 to $48.86 \%$ ). However, the small amount of Mel lowered this value (from 21.21 to 10.28).

\section{XRPD measurements}

To verify the DSC results, the crystallinity of the physical mixtures and the milled samples was investigated by XRPD analysis. Figure 4 presents the fingerprints of raw Mel and PVA and the milled samples. The characteristic peaks of Mel are at $2 \Theta$ value: $13.06^{\circ}, 14.94^{\circ}$ and $18.61^{\circ}$, and PVA has the typical diffraction peak at $2 \Theta$ value: $19.9^{\circ}$ [15]. 
Fig. 4 XRPD curves of raw Mel, PVA and different Melcontaining milled samples

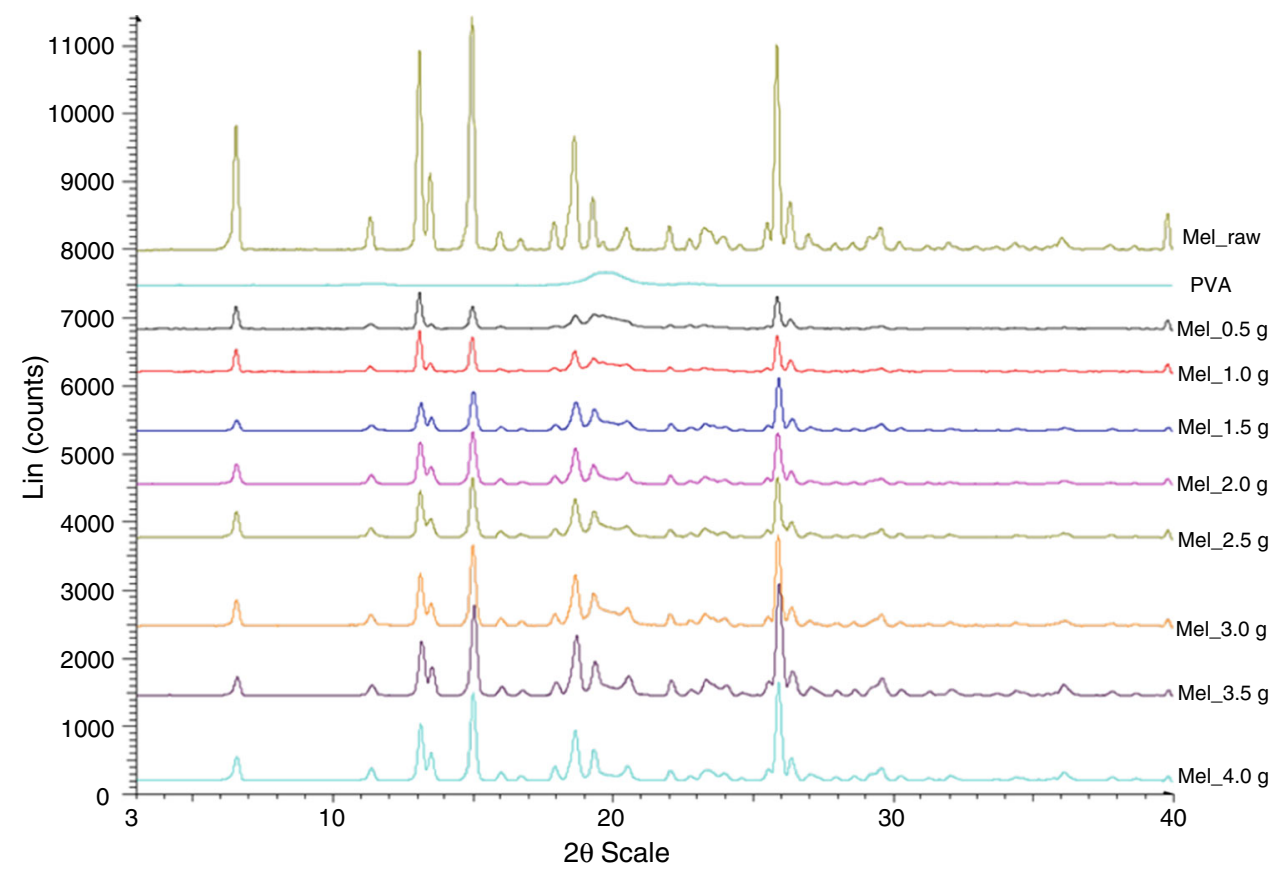

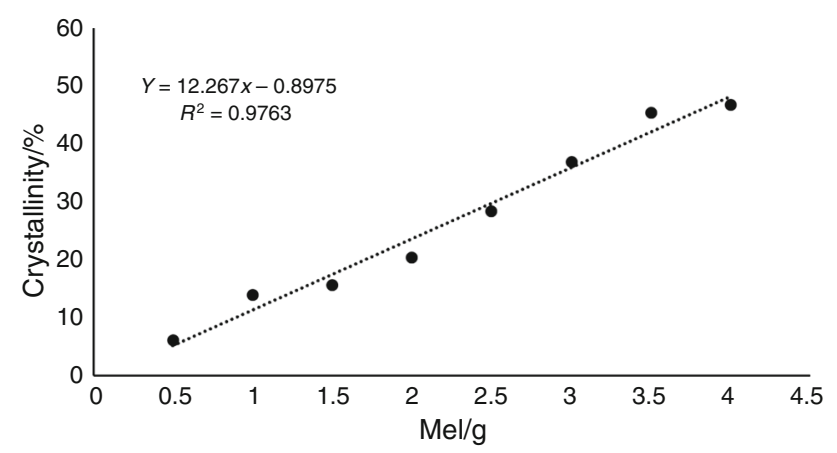

Fig. 5 Relation between the crystallinity of Mel and its amount in the milled samples measured by XRPD

The intensity of the characteristic peaks shows a change in the crystallinity of the milled products. The quantitative analysis of the degree of crystallinity also resulted in a close correlation $\left(R^{2}=0.9763\right)$ between the degree of crystallinity and the Mel amount (Fig. 5).

\section{Conclusions}

In this work, the interval of the Mel amount which can be successfully nanonized $(100-500 \mathrm{~nm})$ was determined by the optimized wet milling process, where the planetary ball mill was combined with pearl milling technology. To discover the robustness of the optimized process, the influence of the amount of Mel was investigated on the particle size distribution and the degree of crystallinity.

The comparison was based on the $10 \%$ of $\mathrm{Mel}(\mathrm{w} / \mathrm{w})$ and 5\% of PVA (w/w) containing sample (Mel_2.0 g) milled by using the following process parameters: 1:1 ratio of pre-suspension and pearls, $437 \mathrm{rpm}$ and $43 \mathrm{~min}$. The milled sample resulted in the expected particle size range, and the degree of crystallinity of Mel was decreased to $20 \%$.

Based on our current results, it can be stated that the increase in the Mel amount ( $>10 \%)$ helped milling efficiency without increasing the amount of milling media (pearls), and the smaller concentration of $\mathrm{Mel}(<10 \%) \mathrm{did}$ not provide the desired particle size distribution. In the first case, the invested mechanical energy was transferred to reduce the particle size, while in the second case to break the crystal structure.

The presence of PVA also affected the degree of particle size reduction. It was found that the milling effectiveness of a lower concentration of PVA $(<5.0 \%)$ provided sufficient protective effect against the aggregation of nanoparticles in the case of samples with $10.0-17.5 \%$ of Mel concentration. This protective effect was not satisfactory (aggregation was observed) for higher Mel content (20.0\%) and lower PVA concentration (4.44\%). A high concentration of PVA $(>5 \%)$ also resulted in unsatisfactory milling effectiveness because a polymer layer was formed on the surface of the particles, protecting them from fragmentation. 
The degree of crystallinity was basically influenced by the Mel amount, but the PVA content also affected it. By increasing the Mel amount, the crystallinity of the milled product was increased measured by the DSC and XRPD techniques and a close correlation was found between crystallinity and the Mel amount by both of them.

It should be noted that recently the characterization of the investigated materials (e.g., crystallinity) using DSC as a semiquantitative conventional method has become common in scientific research [7-9]. It provides a possibility to predict the crystallinity of the examined materials, but to verify the accuracy of thermoanalytical measurements, the $\mathrm{X}$-ray powder diffraction test is required $[10,11]$. In our work, it was found that the degree of crystallinity of Mel investigated by DSC and XRPD did not show any significant difference at $95 \%$ significance level. It can be related to the low degree of crystallinity $(<50 \%)$. It is known that the advantage of DSC over X-ray diffraction is that high amorphous content can be detected [16].

In this system, for the required particle size range, the Mel amount should be changed between 10.0 and 17.5\% $(w / w)$ and a PVA amount should be used between 5.0 and $4.58 \%(\mathrm{w} / \mathrm{w})$. For product stability, it is recommended to keep the PVA amount below 5.0\% and at the same time the critical water content below $1.5 \%$. In this specified range, the amount of Mel can be changed to design the degree of crystallinity of Mel between 20 and $45 \%$ in the final product as well.

To discover the robustness of the milling process, it should also be considered that the amount of grinding media can be reduced by increasing the amount of the active ingredient. It is an important viewpoint because of the reduction in product loss (removing pearls). The DSC method can be suggested for the quantification of the degree of crystallinity because it can be used safely with high amorphous content.

Acknowledgements Open access funding provided by University of Szeged (SZTE). This work was financially supported by Gedeon Richter's Talentum Foundation and Ministry of Human Capacities, Hungary Grant 20391-3/2018/FEKUSTRAT.

Open Access This article is distributed under the terms of the Creative Commons Attribution 4.0 International License (http://creative commons.org/licenses/by/4.0/), which permits unrestricted use, distribution, and reproduction in any medium, provided you give appropriate credit to the original author(s) and the source, provide a link to the Creative Commons license, and indicate if changes were made.

\section{References}

1. Merisko-Liversidge E, Liversidge GG. Nanosizing for oral and parenteral drug delivery: a perspective on formulating poorlywater soluble compounds using wet media milling technology. Adv Drug Deliv Rev. 2011;63:427-40.

2. Junghanns JUA, Müller RH. Nanocrystal technology, drug delivery and clinical applications. Int $\mathrm{J}$ Nanomed. 2008;3:295-309.

3. Shegokar R, Müller RH. Nanocrystals: industrially feasible multifunctional formulation technology for poorly soluble actives. Int J Pharm. 2010;399:129-39.

4. Loh ZH, Samanta AK, Heng PWS. Overview of milling techniques for improving the solubility of poorly water-soluble drugs. Asian J Pharm Sci. 2015;10:255-74.

5. Jójárt-Laczkovich O, Szabó-Révész P. Amorphization of a crystalline active pharmaceutical ingredient and thermoanalytical measurements on this glassy form. J Therm Anal Calorim. 2010;102:243-7.

6. Einfalt T, Planinek O, Hrovat K. Methods of amorphization and investigation of the amorphous state. Acta Pharm. 2013;63:305-34.

7. Katona G, Sípos P, Frohberg P, Ulrich J, Szabó-Révész P, JójártLaczkovich O. Study of paracetamol-containing pastilles produced by melt technology. J Therm Anal Calorim. 2016;123:2549-59.

8. Gieszinger P, Csóka I, Pallagi E, Katona G, Jójárt-Laczkovich O, Szabó-Révész P, Ambrus R. Preliminary study of nanonized lamotrigine containing products for nasal powder formulation. Drug Des Dev Ther. 2017;11:2453-66.

9. Bartos C, Pallagi E, Szabó-Révész P, Ambrus R, Katona G, Kiss T, Rahimi M, Csóka I. Formulation of levodopa containing dry powder for nasal delivery applying the quality-by-design approach. Eur J Pharm Sci. 2018;123:475-83.

10. Bartos C, Jójárt-Laczkovich $\mathrm{O}$, Katona $\mathrm{G}$, Budai-Szücs $\mathrm{M}$, Ambrus R, Bocsik A, Gróf I, Deli MA, Szabó-Révész P. Optimization of a combined wet milling process in order to produce poly(vinyl alcohol) stabilized nanosuspension. Drug Des Dev Ther. 2018;12:1567-80.

11. Dávid Á, Benkóczy Z, Ács Z, Greskovits D, Dávid ÁZ. The theoretical basis for scaling-up by the use of the method of microwave granulation. Drug Dev Ind Pharm. 2000;26:943-51.

12. Li Q, Wang K, Zheng Y, Ruan M, Mei X, Lin B. Experimental research of particle size and size dispersity on the explosibility characteristics of coal dust. Powder Technol. 2016;292:290-7.

13. Perfetti G, Alphazan T, Wildeboer WJ, Meesters GMH. Thermophysical characterization of Pharmacoat ${ }^{\circledR} 603$, Pharmacoat ${ }^{\circledR} 615$ and Mowiol ${ }^{\circledR}$ 4-98. J Therm Anal Calorim. 2012;109:203-15.

14. McNeill IC, Ahmed S, Memetea LT. Thermal degradation of vinyl acetate-methacrylic acid copolymer and the homopolymers. II. Thermal analysis studies. Polym Degrad Stabil. 1995;48:89-97.

15. Garcia-Cerda LA, Escareno-Castro MU, Salazar-Zertuche M. Preparation and characterization of polyvinyl alcohol-cobalt ferrite nanocomposites. J Noncryst Solids. 2007;353:808-10.

16. Gombás Á, Szabó-Révész P, Kata M, Regdon G Jr., Erős I. Quantitative determination of crystallinity of $\alpha$-lactose monohydrate by DSC. J Therm Anal Calorim. 2002;68:503-10.

Publisher's Note Springer Nature remains neutral with regard to jurisdictional claims in published maps and institutional affiliations. 


\section{PUBLICATION IV.}


1

2 Csaba Bartos et al of

meloxicam containing

4 nanosuspension into surfactant-free solid compositions

\section{5 to increase the product stability and drug bioavailability}

\section{6 for rapid analgesia}

7 Bartos Csaba ${ }^{1}$, Rita Ambrus ${ }^{1}$, Gábor Katona ${ }^{1}$, Tamás Sovány $^{1}$, Róbert Gáspár $^{2}$, Árpád Márki ${ }^{3}$,

8 Eszter Ducza ${ }^{4}$, Anita Ivanov ${ }^{4}$, Ferenc Tömösi ${ }^{5}$, Tamás Janáky ${ }^{5}$, Piroska Szabó-Révész ${ }^{1}$

9

$10{ }^{1}$ Faculty of Pharmacy, Institute of Pharmaceutical Technology and Regulatory Affairs, University

11 of Szeged, Szeged, Hungary;

$12{ }^{2}$ Faculty of Medicine, Department of Pharmacology and Pharmacotherapy, University of Szeged, Szeged, Hungary;

$14{ }^{3}$ Faculty of Medicine, Department of Medical Physics and Informatics, University of Szeged,

15 Szeged, Hungary;

$16{ }^{4}$ Faculty of Pharmacy, Department of Pharmacodynamics and Biopharmacy, University of

17 Szeged, Szeged, Hungary;

$18{ }^{5}$ Interdisciplinary Excellence Centre, Department of Medical Chemistry, University of Szeged, Szeged, Hungary

Email

Correspondence: Piroska Szabó-Révész of Szeged, Eötvös u 6, Szeged H-6720, Hungary 
2 Purpose: The aim of this work was to study the influence of solidification of meloxicam (Mel) containing nanosuspension (nanoMel) on the physical stability and drug bioavailability of the products. The nanoMel sample had poly(vinyl alcohol) (PVA) as a protective polymer, but no surfactant as a further stabilizing agent because the final aim was to produce a surfactant-free solid phase products as well.

Methods: The solidified samples produced by fluidization and lyophilization (fluidMel, lyoMel) were examined for particle size, crystallinity, and in vitro release of Mel compared to similar parameters of nanoMel. The products were subjected to an animal experiment using per oral administration to verify their bioavailability.

Results: Mel containing (1\%) nanoMel sample was produced by wet milling process using optimized amount of PVA (0.5\%) which resulted in $130 \mathrm{~nm}$ as mean particle size and a significant reduction in the degree of crystallinity (13.43\%) of Mel. The fluidization technique using microcrystalline cellulose (MCC) as carrier resulted a quick conversion no significant change in the critical product parameters. Process of lyophilization required a longer operation time, which resulted in the amorphization of the crystalline carrier (trehalose) and the recrystallization of Mel increased its particle size and crystallinity. The fluidMel and lyoMel samples had nearly five-fold higher relative bioavailability than nanoMel application by oral administration. The correlation between in vitro and in vivo studies showed that the fixed Mel nanoparticles on the surface of solid carriers (MCC, trehalose) in both the artificial gastric juice and the stomach of the animals rapidly reached saturation concentration leading to faster dissolution and rapid absorption.

Conclusion: TThe solidification of the nanosuspension not only increased the stability of the Mel nanoparticles, but also allowed the preparation of surfactant-free compositions with excellent bioavailability which may be an important consideration for certain groups of patients to achieve rapid analgesia.

Keywords: solidification, fluidization, lyophilization, surfactant-free product, rapid drug absorption, IVIV correlation 


\section{Introduction}

2 Nanosuspensions can be defined as colloidal dispersions of nanosized drug particles $(<500 \mathrm{~nm})$

3 that are produced by different nanonization processes and stabilized by various excipients. ${ }^{1}$

4 Nanonization of drugs with different top-down methods (wet-bead milling, high-pressure

5 homogenization and microfluidization) is a proven effective strategy to decrease the particle size

6 by mechanical processes and to enhance the dissolution rate, saturation solubility and

7 bioavailability of poorly water-soluble active ingredients, such as BCS class II (poorly soluble and

8 high permeable) and Class IV (poorly soluble and permeable). ${ }^{2,3}$ Nanosuspensions produced by

9 milling are generally unstable, therefore stabilizing agents (polymers, surfactants) and its

10 transformation to the solid state have an important role in the formulations with long-term

11 stability. ${ }^{4,5}$ Water-soluble polymers, such as $2.4-19.6 \%$ of cellulose ethers, ${ }^{6} 30 \%$ of poly(vinyl

12 pyrrolidone), ${ }^{7,8}$ and $50 \%$ of poly(vinyl alcohol) $)^{9,10}$, are mainly used in wet milling. The most

13 commonly used surfactants and their amount in relation to the amount of active ingredient are as

14 follows: Cremophor ${ }^{R}(100 \%),{ }^{11}$ Poloxamer $188(60 \%),{ }^{12}$ Poloxamine $908(20 \%),{ }^{13}$ Tyloxapol (20

$15 \%),{ }^{14}$ sodium lauryl sulfate $(0.15 \%),{ }^{15}$ and Polysorbate $80(1 \%) .{ }^{16,17}$ In the absence of stabilizers,

16 the high surface energy of nanosized drug particles can induce aggregation/agglomeration in the

17 system. ${ }^{18}$ The main functions of a stabilizers in nanosuspensions are to wet drug particles during

18 the milling process, and to prevent Ostwald's ripening (crystal growth in colloidal suspensions) ${ }^{19}$

19 and agglomeration in order to yield a physically stable formulation by providing steric or ionic

20 barriers. Different concentrations of stabilizer agents (e.g. polymers) can also influence the

21 viscosity and the electro-kinetic property of the particles, according to the DLVO theory, ${ }^{20}$ and

22 thus the stability of the nanosuspension as well. Surfactants help to wet the particles and thus reduce their aggregation tendency. In addition to the advantages of surfactants, they have the biggest disadvantage of increasing the speed/energy of motion of the milling balls during wet milling, which can lead to the degradation of the active ingredient. When used as an external surfactant to solidify the nanosuspension, its solubility-enhancing effect may be emphasized, thereby increasing the degree of crystallinity of active agent in the solid product and reducing its dissolution rate. ${ }^{21}$ Conventional formulations contain these excipients in common, but the new 
1 tendency is to ignore the surfactants and look for other options to stabilize the nanoparticles in

2 the products and achieve the desired biological effect. $22-24$

3

4

5

6

7

8

Crystalline state is one of the most important parameters affecting drug stability, dissolution extent, and efficacy. The high energy wet milling techniques tend to create a partially amorphous active agent. The high energy amorphous particles are unstable, especially in the presence of crystalline particles, and inclined to convert to low energy crystalline state over time.

The saturation solubility between amorphous and crystalline nanoparticles is different, therefore the diffusion process will be similar to Oswald's ripening, leading to a rapid conversion of amorphous nanoparticles to crystalline state. ${ }^{25}$

Of course, the nanosuspensions can be applied as final liquid dosage forms using further different excipients (viscosity enhancer, flavoring, preservative agents, etc.), however, their stabilization is a major challenge. ${ }^{26}$ It is well known that, despite the stabilization, nanosuspensions have a short expiration time, and there are patients who do not prefer this form or the presence of a surfactant. One way to overcome the instability and surfactant problem is to design solid nanosuspension produced by spray drying, spray freeze drying and freeze drying (lyophilization). It is well known that the dry nanosuspensions can cause difficulty in hydration and redispersibility ${ }^{24,27}$. Other processes for transforming a nanosuspension into a solid-state forms (tablets, capsules) are very different: deposition as coatings, incorporation in granules and pellets and the 3D printing technologies. ${ }^{28}$

Spray drying and lyophilization (freeze drying) are the commonly used techniques for the solidification of nanosuspension because of their easy application and industrial acceptability. ${ }^{29}$ The powders produced by these processes often suffer from poor flowability and high hygroscopicity, therefore other technologies are applied to transform nanosuspensions into oral dosage forms as tablets and capsules. ${ }^{30}$

Layering of nanosuspension onto the surface of granules, pellets, sugar beads, etc. using a fluidization technique is used as an alternative method for solidification of nanosuspension. ${ }^{30-32}$ The advantage of this process that may be used various additives in order to achieve the desired purpose, e.g. i) different polymers stabilize the nanosuspension, and act as a coating materials resulted in fast dissolution rate, ii) surfactants prevent the aggregation of nanoparticle and can 
1

2

3

4

5

6

7

8

9

modify the drug release. ${ }^{33}$ In any case, the fluidization technique also provides an opportunity to stabilize the broken structure of the ground crystals in the nanosuspension by using crystallization inhibitors. $^{34}$

Mel, a member of the oxicam family of NSAIDs (non-steroidal anti-inflammatory drugs), as a moderately selective cyclooxygenase (COX-2) inhibitor can have a role in acute pain therapy but a basic requirement is rapid absorption through the gastric mucosa. Mel has a weak acidic character with pKa of 3.43 , therefore its solubility in gastric juice $(\mathrm{pH}=1.2)$ is very poor but its logPapp is $2.43(\mathrm{pH}=2.0)$, which predestines the fast absorption from the stomach. ${ }^{35-37}$ Since the solubility of Mel is very poor in gastric juice, the preparation of a nanosuspension with a fast dissolution rate may be a solution.

In our previous work we developed a wet milling procedure for the nanonization of Mel, which was reported in the DDDT in $2018 .^{10}$ We optimized the critical process parameters by factorial design (ratio of predispersion and pearls, milling time and rotation speed of the steel jar) and investigated the PVA amount on the particle size distribution and crystallinity of the Mel. The optimized process parameters and PVA amount have allowed the use of no surfactant during milling to prevent aggregation. We had also the surfactant free nanosuspension as intermediate product showed a stable system for critical product parameters with 2 weeks of holding time. Mel containing nanosuspension. Critical product parameters were considered to be the particle size distribution of the drug $(\mathrm{d}(0.9)<500 \mathrm{~nm})$, stabilization of the degree of crystallinity altered during milling, and enhancement of the bioavailability of the solid product with fast absorption from the stomach for rapid analgesia. The transformation of the nanosuspension was done by fluidization and lyophilization.

\section{Materials and Methods}

\section{Materials}


1 Mel (rawMel) was obtained from EGIS Ltd. (Budapest, Hungary). PVA-Mowiol ${ }^{R}$ 4-98 (Mw

227,000 ) (Sigma Aldrich Co. LLC, St. Louis MO, USA) was used as a stabilizing agent. Zirconium 3 oxide $\left(\mathrm{ZrO}_{2}\right)$ beds with a diameter of $0.30 \mathrm{~mm}$ were obtained from Netsch (Netsch $\mathrm{GmbH}$, Selb,

4 Germany). Microcrystalline cellulose (MCC) (Avicel ${ }^{R}$ PH 101, FMC Biopolymer, Philadelphia

5 USA) was used as a carrier material for the fluidized product. D-(+)-trehalose dihydrate as a cake-

6 forming agent was purchased from Karl Roth GmbH + Co. KG. (Karlsruhe, Germany).

7

$8 \quad$ Methods

$9 \quad$ Preparation of nanosuspension (nanoMel)

10 For the production of the Mel nanosuspension, a planetary ball mill was combined with pearl milling technology. PVA was used as a stabilizing additive, $1.0 \mathrm{~g}$ of PVA was dissolved in $17.0 \mathrm{~g}$ of distilled water as a dispersant medium, in which $2.0 \mathrm{~g}$ of Mel was suspended. The milling was executed using a Retsch PM 100 planetary ball mill (Retsch GmbH, Haan, Germany) at 437 rpm rotation speed for $43 \mathrm{~min}$ in addition with $20.0 \mathrm{~g}$ of $0.3 \mathrm{~mm} \mathrm{ZrO} 2$ beads as a milling medium. After the milling process, to eliminate the grinding medium from the sample, a $0.150-\mathrm{mm}$ sieve was used. The nanosuspension was removed from the beads by cleaning with distilled water, while the milled sample was ten-fold diluted. The yield of the milling process was $94.93 \%$. The final concentration of the components can be seen in Table 1.

Table 1 Composition of the investigated samples

\begin{tabular}{|c|c|c|c|c|c|}
\hline Sample & Mel & PVA & Water & MCC & Trehalose \\
& $(\%)$ & $(\%)$ & $(\%)$ & $(\%)$ & $(\%)$ \\
\hline nanoMel & 1.00 & 0.50 & 98.50 & - & - \\
\hline fluidMel $^{\mathrm{a}}$ & 1.94 & 0.97 & - & 97.09 & - \\
\hline lyoMel $^{\mathrm{b}}$ & 15.38 & 7.70 & - & - & 76.92 \\
\hline
\end{tabular}

Notes: ${ }^{\mathrm{a}, \mathrm{b}}$ based on dry material 


\section{Transformation of nanosuspension into solid state form}

2 In preformulation study, surfactant-free and external surfactant-containing (Polysorbate 80)

3 samples were produced by fluidization to study the critical parameters of the products. It was

4 found that the external surfactant used to solidify the nanosuspension (nanoMel) increased the

5 degree of crystallinity and decreased the dissolution rate of Mel. This change is related to the

6 solubility-enhancing effect of the surfactant. It was concluded that, in the absence of surfactant,

7 the critical product parameters can be fulfilled by optimization of process parameters of fluidization

8 and lyophilization.

9

10 Fluidization (fluidMel)

11 MCC as the carrier material was used in a Strea-1 (Niro Aeromatic, Bubendorf, Switzerland) fluid bed chamber. A batch size of $100.0 \mathrm{~g}$ was used. The powder was inserted and fluidized in the preheated chamber for a period of $10 \mathrm{~min}$ and at constant air velocity of $2.5 \mathrm{~m} / \mathrm{s}$. NanoMel as liquid dispersion was transported by a peristaltic pump (Roto Consulta, Ebikon/Luzern, Schweiz), the applied pump speed was $9 \mathrm{rpm}$. One batch of $200.0 \mathrm{~g}$ of nanoMel dispersion was atomized onto the surface of the material. The process took 50 minutes, the inlet temperature was $55^{\circ} \mathrm{C}$ and the outlet temperature was $38^{\circ} \mathrm{C}$. The final concentration of the components in percentage is shown in Table 1. The yield of the process was calculated based on the proportion of the mass of the components before and after operation.

\section{Lyophilization (lyoMel)}

Freeze-drying was performed in Scanvac CoolSafe 100-9 Pro type equipment (LaboGene ApS, Lynge, Denmark) equipped with a 3-shelf sample holder unit, recessed into the drying chamber. In each cuvette $750 \mathrm{mg}$ of diluted milled suspension was filled $(7.5 \mathrm{mg}$ Mel content in every cuvette, the unit dose of Mel). As a cake-forming additive, $37.5 \mathrm{mg}$ of $(5.0 \%)$ trehalose was dissolved in the nanosuspensions. The components of the final product can be seen in Table 1. The process was controlled by a computer program (Scanlaf CTS16a02), the temperature and pressure values were recorded continuously. The whole process took 71 hours and 52 minutes. 
1 The initial temperature was $25^{\circ} \mathrm{C}$. During the freezing period, after 18 hours and 34 minutes, the

2 sample temperature was decreased to $-40{ }^{\circ} \mathrm{C}$. The subsequent drying process was conducted at

30.013 mbar air pressure for 50 hours and 50 minutes, the temperature increased from $-40{ }^{\circ} \mathrm{C}$ to -

$4 \quad 7^{\circ} \mathrm{C}$. Finally, the secondary drying session took 2 hours and 28 minutes. The final temperature

5 of the dried products was $30^{\circ} \mathrm{C}$.

6

7 Characterization of nanosuspension and the solid state forms

$8 \quad$ Particle size measurements

9 The investigations on the particle size of rawMel el and nanoMel via laser diffraction were executed (Malvern Mastersizer S 2000, Malvern Instruments Ltd, Worcestershire, UK) with the following parameters: 300RF lens; small volume dispersion unit (1,000 rpm); refractive index for Mel: 1.596; and refractive index for dispersion medium: 1.330. During the measurements, distilled water was used as a dispersant, and obscuration was in the range of $11 \%-16 \%$ for all measurements. In both cases, the particle size distributions were qualified by $d(0.1), d(0.5)$, and $d(0.9)(d(0.5)$ is the particle diameter below which $50 \%$ of the sample volume exists). In the case of the solid state products (fluidMel and lyoMel), the particle size of Mel was determined by using Scanning Electron Microscopy (SEM) images (Hitachi S4700, Hitachi Scientific Ltd., Tokyo, Japan). The size of the particles was calculated by ImageJ software for Windows (Phase $\mathrm{GmbH}$, Lübeck, Germany).$^{38}$ By specifying the unit length, which is shown in each image (depending on the magnification this is a different value), the actual particle size can easily be determined by drawing the diameter of the captured particles. A diameter of a hundred captured particles was determined in the case of two solid state samples. For nanoMel and lyoMel samples, the Z-average particle size and the polydispersity index (PDI) of Mel were measured using a Malvern Zeta Nano ZS (Malvern Instruments Ltd). In case of the particle size determination, Malvern DTS 1070 folded capillary cell was used. The samples were further diluted with water (25-fold) for the measurements. 
1 For SEM investigations, nanoMel samples was dried in a vacuum dryer (Binder GmbH, Tuttlingen,

2 Germany) at $40^{\circ} \mathrm{C}$ in order to obtain solid products and fluidMel and lyoMel were visualized as

3

4

5

6

$7 \quad$ Differential scanning calorimetry (DSC)

8 To investigate the occurring physico-chemical changes and to predict the crystallinity of the solid well. The samples were sputter-coated with gold-palladium under an argon atmosphere, using a gold sputter module in a high-vacuum evaporator, and the samples were examined at $10 \mathrm{kV}$ and $10 \mathrm{~mA}$. The air pressure was $1.3-13 \mathrm{MPa}$.

state products, DSC measurements were carried out with a Mettler Toledo DSC 821e thermal analysis system with the STARe thermal analysis software V9.0 (Mettler Inc. Schwerzenbach, Switzerland). Approximately $2-5 \mathrm{mg}$ of the physical mixtures (PM) and the product samples were examined in the temperature range of $25-300{ }^{\circ} \mathrm{C}$. The heating rate was $20^{\circ} \mathrm{C} / \mathrm{min}$ in the presence of argon as a carrier gas with a flow rate of $10 \mathrm{~L} / \mathrm{h}$. The calculations of Mel crystallinity (Cryst \%) were performed using the area under the curve $(A \cup C)$ of the melting enthalpy of the products (AUCMel) and the physical mixtures (AUCMelPM). ${ }^{39}$ The values were compared using the following formula:

$$
\text { Cryst } \%=\frac{\text { AUCMel }}{\text { AUCMelPM }} * 100
$$

\section{Stability test}

The products (fluidMel and lyoMel) were stored in a well-closed container, at room temperature $\left(23 \pm 2{ }^{\circ} \mathrm{C}, 45 \pm 5 \% \mathrm{RH}\right)$ for 6 months. The crystallinity of Mel was investigated compared to freshly measured products.

\section{Drug content determination}

The Mel content of the samples was controlled in the following way. The unit dose of the products with $0.75 \mathrm{mg}$ of theoretical Mel was dissolved in $100 \mathrm{~mL}$ of phosphate buffer $\mathrm{pH} 7.4 \pm 0.1$. The sample was stirred with a magnetic stirrer at $25{ }^{\circ} \mathrm{C}$ for $24 \mathrm{~h}$ and then filtered $(0.1 \mu \mathrm{m}$, FilterBio PES Syringe Filter) (Labex Ltd., Budapest, Hungary), and the concentration of the dissolved Mel 
was analyzed spectrophotometrically (Unicam UV/VIS) (Thermo Fisher Scientific Inc., Waltham, MA, USA) at $364 \mathrm{~nm}$ wavelength. The investigations were repeated three times.

\section{Solubility testing of MEL in the samples}

The solubility of Mel in the samples (nanoMel, fluidMel and lyoMel) was determined. The dispersions were stirred with a magnetic stirrer at $25^{\circ} \mathrm{C}$ for $24 \mathrm{~h}$ and then filtered $(0.1 \mu \mathrm{m}$, FilterBio PES Syringe Filter) (Labex Ltd., Budapest, Hungary), and the dissolved drug content was analyzed spectrophotometrically (Unicam UV/VIS) (Thermo Fisher Scientific Inc., Waltham, MA, USA) at $364 \mathrm{~nm}$ wavelength $(\mathrm{n}=3)$.

\section{In vitro dissolution test}

To determine the dissolution extent of Mel from different products, the paddle method (USP dissolution apparatus, type II Pharma Test, Heinburg, Germany) was used. The medium was 900 $\mathrm{ml}$ of artificial gastric fluid at $\mathrm{pH} 1.2 \pm 0.1$. The paddle was rotated at $100 \mathrm{rpm}$ and sampling was performed up to $120 \mathrm{~min}$. The Mel content of the samples was determined with a spectrophotometer (ATI-UNICAM UV/VIS Spectrophotometer, Cambridge, UK) at $362 \mathrm{~nm}$. The number of parallels was three.

\section{Statistical analyses}

Data from the above methods were expressed as means $\pm S D$, and groups were compared by using Student's t-test. Differences were considered statistically significant when $p<0.05$.

\section{In vivo studies}

All experiments involving animal subjects were carried out with the approval of the National Scientific Ethical Committee on Animal Experimentation (permission number: IV/1247/2017). The animals were treated in accordance with the European Communities Council Directives (2010/63/EU) and the Hungarian Act for the Protection of Animals in Research (Article 32 of Act XXVIII). Each sample contained $60 \mu \mathrm{g} / \mathrm{ml}$ of Mel and $30 \mu \mathrm{g} / \mathrm{ml}$ of PVA in distilled water. For per 
os delivery, the different formulations were individually diluted and were given at a single dose of $300 \mu \mathrm{g} / \mathrm{kg}$ of Mel to male Sprague-Dawley rats ( 8 weeks old, $240-260 \mathrm{~g}, \mathrm{n}=6$ ) in a volume of 0.5 $\mathrm{ml}$ by gastric gavages. All animals fasted 16 hours before the per os administration of drugs. In order to facilitate the absorption, the solid-state forms were re-dispersed in water immediately before administration. In a comparison study for intravenous administration, animals were treated with a $300 \mu \mathrm{g} / \mathrm{kg}$ bolus of Mel via the tail vein.

Intravenous (IV) injection was prepared by the dilution of passable injection with a concentration of $15 \mathrm{mg} / 1.5 \mathrm{~mL}$ (Meloxicam-Zentiva, Prague, Czech Republic) to reach the final concentration $(0.15 \mathrm{mg} / \mathrm{mL})$. The ingredients of the injection were meglumine, poloxamer 188 , glycine, sodium hydroxide (for $\mathrm{pH}$ adjustment), sodium chloride, glycopherol, and water for injection. Blood samples were collected from the tail vein before and at 15, 30, 60, 75, 90, 120 and 180 minutes post-dosing. Plasma samples were collected into EDTA containing polyethylene tubes, centrifuged at $1,500 \mathrm{~g}$ for $10 \mathrm{~min}$ at $5{ }^{\circ} \mathrm{C}$. Separated plasma samples were stored at $-80^{\circ} \mathrm{C}$ until extraction and analysis.

\section{Determination of Mel from rat plasma}

Preparation of plasma samples, calibration standards and quality control samples

To $90 \mu \mathrm{L}$ of plasma sample, $10 \mu \mathrm{L}$ of $0.1 \%$ aqueous formic acid and $300 \mu \mathrm{L}$ of acetonitrile containing piroxicam (internal standard at $12.5 \mathrm{ng} / \mathrm{mL}$ concentration) were added and the mixture was vortex-mixed for $60 \mathrm{~s}$. The mixture was allowed to rest for $30 \mathrm{~min}$ at $-20^{\circ} \mathrm{C}$ to support protein precipitation. Supernatant was obtained by the centrifugation of the mixture for $10 \mathrm{~min}$ at 10,000 g at $4{ }^{\circ} \mathrm{C}$ and $20 \mu \mathrm{L}$ was diluted with $380 \mu \mathrm{L}$ of $0.1 \%$ aqueous formic acid. Finally, $5 \mu \mathrm{L}$ was injected into the LC-MS/MS system for analysis.

Rat plasma calibration standards of meloxicam were prepared by spiking the working standard solutions (1-1000 $\mathrm{ng} / \mathrm{mL})$ into a pool of drug-free rat plasma and the procedure described above was followed. Calibration standards consisted of $90 \mu \mathrm{L}$ of pooled drug-free plasma, $10 \mu \mathrm{L}$ of meloxicam standard solution (in $0.1 \%$ aqueous formic acid) and $300 \mu \mathrm{L}$ of acetonitrile containing piroxicam (internal standard at $12.5 \mathrm{ng} / \mathrm{mL}$ concentration). Solutions containing $6.25 \mathrm{ng} / \mathrm{mL}$ and $25 \mathrm{ng} / \mathrm{mL}$ of meloxicam were used as QC samples. $20 \mu \mathrm{L}$ of supernatant was taken out from both 
1 of the calibration standards and the QC samples, diluted with $380 \mu \mathrm{L}$ of $0.1 \%$ aqueous formic 2 acid, and $5 \mu \mathrm{L}$ was analyzed by LC-MS/MS.

3

4 LC-MS/MS analysis of meloxicam

5 The quantitative analysis of meloxicam was performed by mass spectrometry after the 6 chromatographic separation of analytes. An Agilent Liquid Chromatography System series 1100 7 (Micro Vacuum Degasser, Capillary Pump, $\mu$-WPS autosampler) (Agilent Technologies, 8 Waldbronn, Germany) was connected to a Q ExactiveTM Plus Orbitrap mass spectrometer 9 (Thermo Fisher Scientific, San Jose, US) equipped with a heated ESI ion source. Gradient 10 chromatographic separation was performed at room temperature on a Luna $5 \mu \mathrm{m} C 8(2)$ Mercury 11 column $(20 \mathrm{~mm} \times 2.0 \mathrm{~mm})$ protected by a C8 guard column (2x2 mm) (Phenomenex, Torrance, 12 USA) using ammonium formate $(15 \mathrm{mM}, \mathrm{pH}=3)$ as Solvent $\mathrm{A}$ and acetonitrile as Solvent $\mathrm{B}$ (Table 13 2). The calibration curve was shown to be linear over the concentration range of $1-1000 \mathrm{ng} / \mathrm{mL}$

Table 2 The gradient elution program applied for analysis

\begin{tabular}{|c|c|c|}
\hline $\mathrm{t}(\mathrm{min})$ & $\mathrm{B}(\%)$ & Flow rate $(\mu \mathrm{L} / \mathrm{min})$ \\
\hline 0 & 40 & 250 \\
\hline 0.5 & 40 & 250 \\
\hline 2 & 70 & 250 \\
\hline 2.1 & 90 & 600 \\
\hline 2.5 & 90 & 600 \\
\hline 2.6 & 40 & 600 \\
\hline 4.0 & 40 & 600 \\
\hline 4.1 & 40 & 250 \\
\hline 4.5 & 40 & 250 \\
\hline
\end{tabular}


Abbreviation: B, solvent (acetonitrile)

The mass spectrometer was used in positive mode with the following parameters of $\mathrm{H}$ ESI source: ion spray voltage at $3.5 \mathrm{kV}$, capillary temperature at $253^{\circ} \mathrm{C}$ and aux gas heater temperature at $406{ }^{\circ} \mathrm{C}$, sheath gas flow rate at $46 \mathrm{I} / \mathrm{h}$, aux gas flow rate at $11 \mathrm{l} / \mathrm{h}$, sweep gas flow rate at $2 \mathrm{l} / \mathrm{h}$ and S-lens RF level at 50.0 (source auto-defaults). Multiple-reaction-monitoring (MRM) mode was used for quantification by monitoring the transitions: $\mathrm{m} / \mathrm{z} 352 \rightarrow 115$ and $352 \rightarrow 141$ for meloxicam (collision energy $24 \mathrm{~V}$ ) and $\mathrm{m} / \mathrm{z} 332 \rightarrow 95$ and $332 \rightarrow 121$ for piroxicam (collision energy 29V). A divert valve placed after the analytical column was programmed to switch flow onto MS only when analytes of interest elute from the column (plasma samples: 0.7-2.0 min) to prevent the excessive contamination of the ion source and ion optics.

Data acquisition and processing were carried out using Xcalibur and Quan Browser (version 4.0.27.19) software (Thermo Fisher Scientific, San Jose, US).

The area under the curve $(\mathrm{AUC})$ of the time $(\mathrm{min})$-concentration $(\mathrm{ng} / \mathrm{ml})$ curves of each animal and the statistical analysis were performed with Prism 5.0 software (GraphPad Software Inc., La Jolla, CA, USA). All data presented are means \pm SD. The unpaired t-test was used to determine statistical significance. Changes were considered statistically significant at $p<0.05$. The ratio of AUC value, after the per oral application of the transformed samples (AUCfluidMel, AUClyoMel) in comparison with the AUC of the peroral application of nanoMel (AUCnanoMel) as relative bioavailability (rel.BA) was determined according to the formula below. ${ }^{40}$

$$
\text { rel.BA for plasma }(\%)=\frac{\text { AUCtransformed sample }}{\text { AUCnanoMel }} * 100
$$

\section{In vitro-in vivo correlation calculation}

In vitro-in vivo correlation (IVIVC) is a biopharmaceutical tool for the investigation of the mutual relationship of the dissolution characteristics of the in vitro and in vivo absorption studies. $41 \mathrm{In}$ our case, the Pearson's correlation coefficient of the AUC values of the in vitro and in vivo results was calculated by Microsoft Excel (Microsoft Corporation, Redmond, Washington, U.S.) and Statistica for Windows (StatSoft GmbH, Hamburg, Germany). The three prepared samples were 
1 correlated with each other in groups of in vitro and in vivo. To determine statistical significance,

2 the unpaired t-test was used.

3

\section{Results and discussion}

\section{$5 \quad$ Particle size measurements}

6 Different techniques have been used to determine the particle size of Mel for reasons of accuracy

7 and comparability. The particle size distribution of the rawMel and nanoMel samples was

8 investigated via laser diffraction. A combined wet milling process resulted in a 200 -fold particle

9 size reduction in the case of nanoMel $(\mathrm{d}(0.50), 130 \pm 5 \mathrm{~nm})$ compared to the raw drug particle size

$10(\mathrm{~d}(0.50), 34.26 \pm 4.86 \mu \mathrm{m})$. The nanoMel product showed a monodisperse distribution $(\mathrm{d}(0.10)$,

$1167 \pm 1 \mathrm{~nm} ; \mathrm{d}(0.50), 130 \pm 5 \mathrm{~nm} ; \mathrm{d}(0.90), 371 \pm 12 \mathrm{~nm})$.

For fluidMel sample, Mel particles adhered to the carrier surface (MCC) was analyzed by Image J technique and the particle size of Mel in the nanoMel and lyoMel samples was compared with dynamic light scattering technique (Malvern nanoZS), too. The results demonstrate that the $d(0.50)$ value of the Mel nanoparticles measured on the surface of solid phase product (fluidMel) does not show a significant difference regarding the $d(0.50)$ value of the nanoMel (Figure 1a). In the case of lyoMel, compared to the Z-average of Mel in nanoMel, a significant difference can already be detected which was caused by the recrystallization of the Mel (Figure 1b). Both samples have a same polydispersity index (nanoMel: 0.273 and lyoMel: 0.287 ) which also confirms the monodispersity of nanoMel and shows the excellent redispersibility of lyoMel. The (Figure 1a, 1b). 


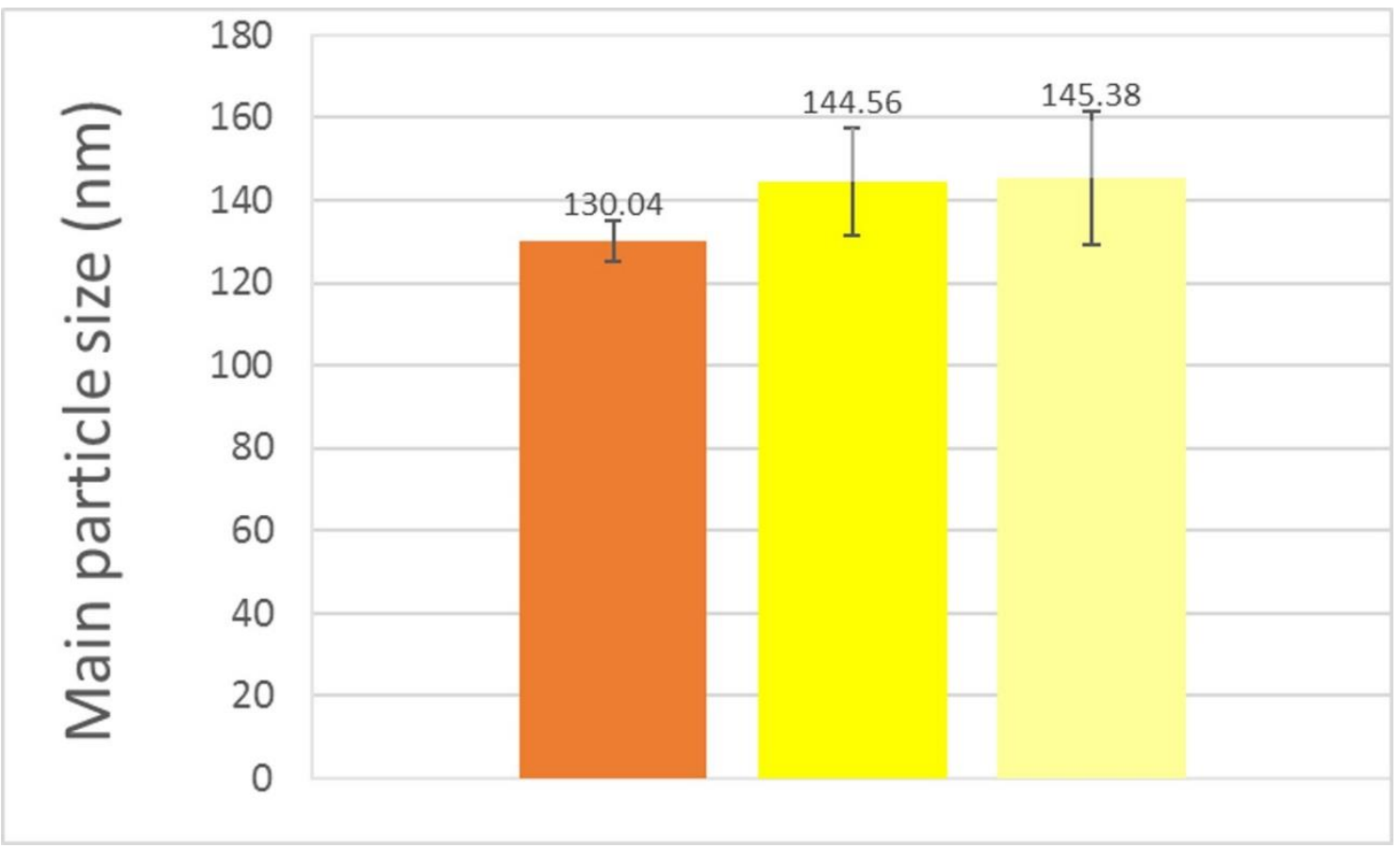

2

(A)

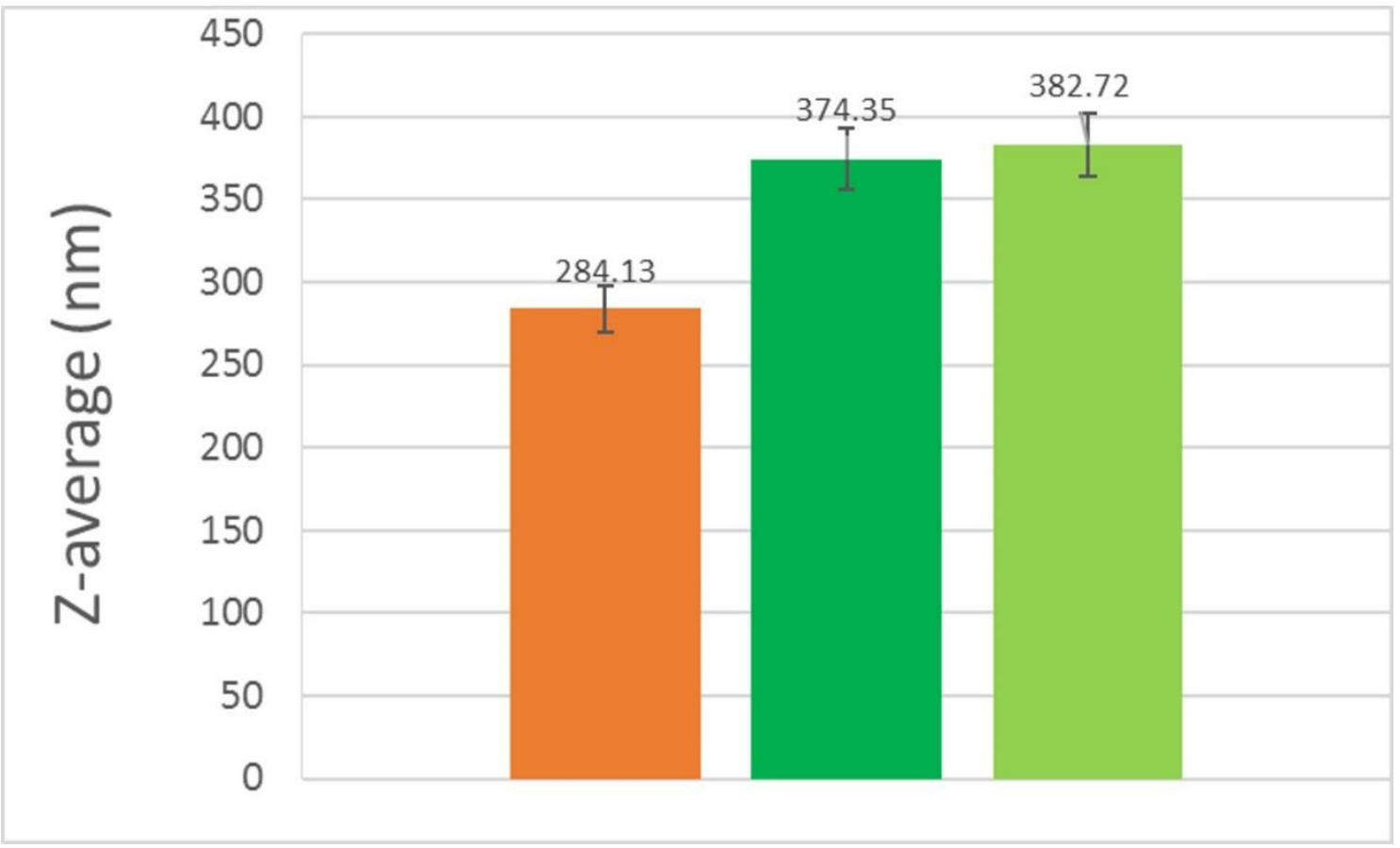

(B)

$5 \quad$ Figure 1 (from left to the right)

6 Main particle size of nanoMel (measured by laser diffraction), fluidMel fresh and fluidMel stored (6

7 months) (measured by SEM images) (A) and Z-average of nanoMel, lyoMel fresh, and lyoMel stored (6

8 months) measured by Zeta nano ZS) (B)

9 Abbreviations: Mel, meloxicam; SEM, scanning electron microscopy 


\section{SEM measurements}

2 Figure $2 \mathrm{~A}$ and Figure $2 \mathrm{~B}$ clearly show the particle size difference between the rawMel and

3 nanoMel and the change in the particle habit. The latter particles have smooth surfaces with

4 rounded edges and corners. High mechanical impact results in the fracture and abrasion of the crystals.
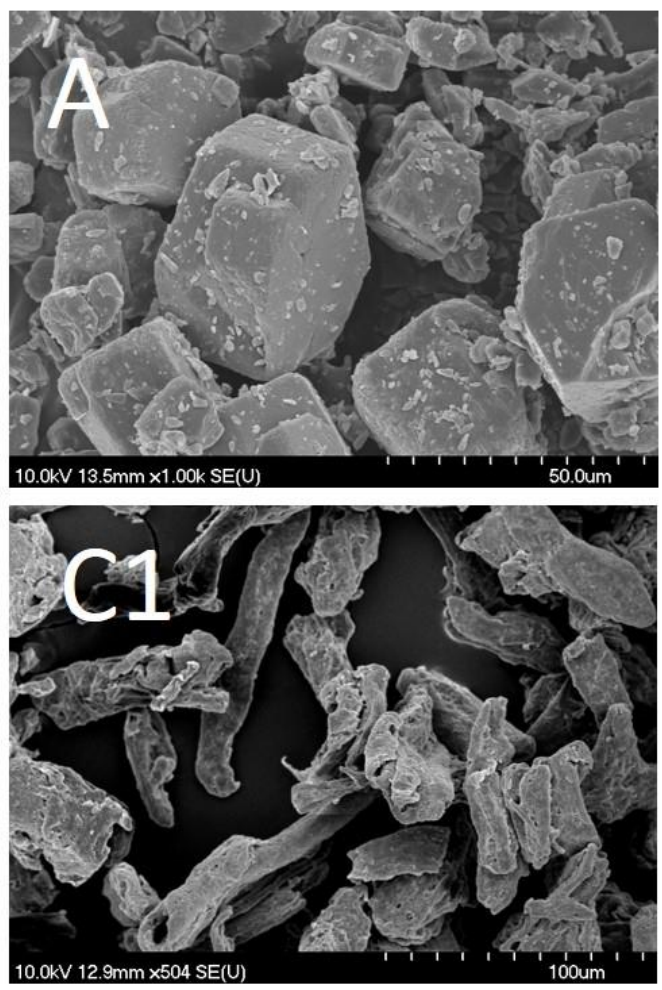

6

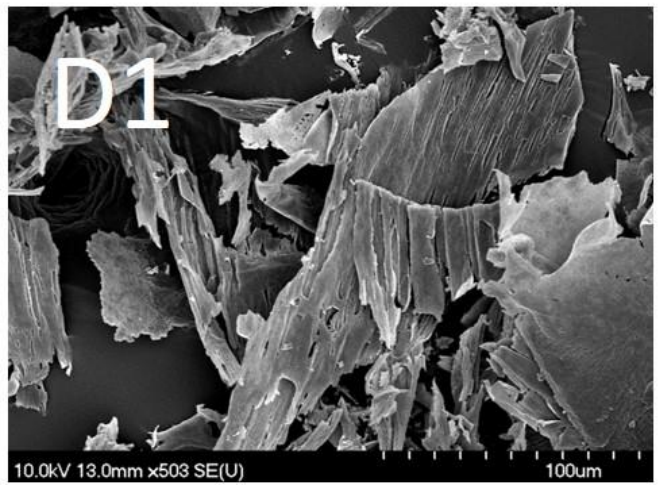

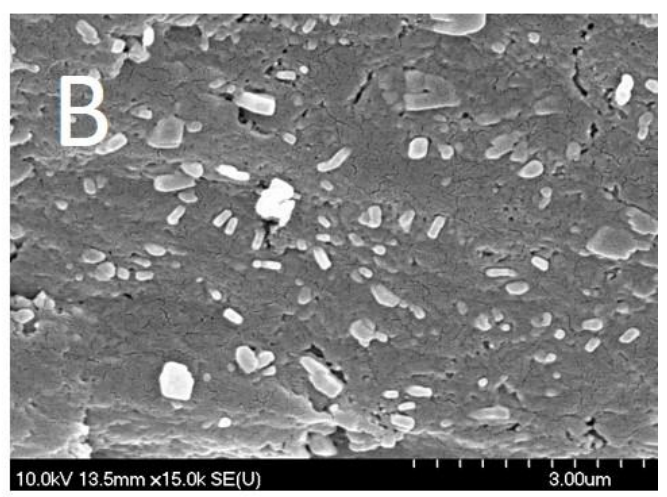
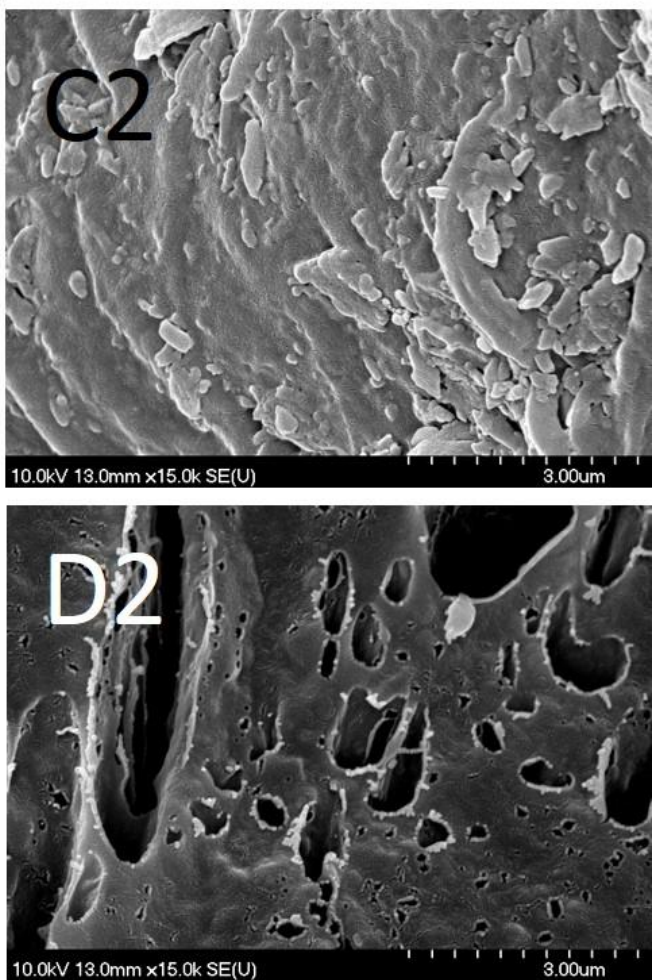

Figure 2 SEM image of rawMel (A), nanoMel (B), fluidMel (C1 and C2) and lyoMel (D1 and D2)

Abbreviations: Mel, meloxicam; SEM, scanning electron microscopy During the fluidization process, the nanoparticles are uniformly adhered to the surface of MCC (Figure 2C1). Their habit is the same as that of the nanoparticles in the nanoMel (Figure 2C2). There is no sign indicating the aggregation of the nanoparticles on the surface of MCC. The adhesion of the Mel particles to 
1 the carrier surface is also supported by the effect of the PVA adhesive property and the rapid 2 evaporation of water.

The SEM image of the lyoMel sample (Figure 2D1) shows large, consistent formulas at

4 low magnification. The texture at higher magnification contains smaller, larger pores resulting in

5 a big surface with honeycombed structure, where the surface area is determined by the size of

6 the ice crystals. ${ }^{42}$ The SEM picture does not show any trehalose-like crystals in the structure

7 (Figure 2D2).

8

\section{DSC measurements, crystallinity determination}

10 The DSC curves of the components and the products are shown in Figure 3. The rawMel has a relative high melting point at $268^{\circ} \mathrm{C}, \mathrm{PVA}$ as semi-crystalline material has two endothermic peak

12 at $169^{\circ} \mathrm{C}$ and at $222^{\circ} \mathrm{C}$. MCC shows any characteristic peak, in contrast the trehalose is a crystalline material (Figure 3a).

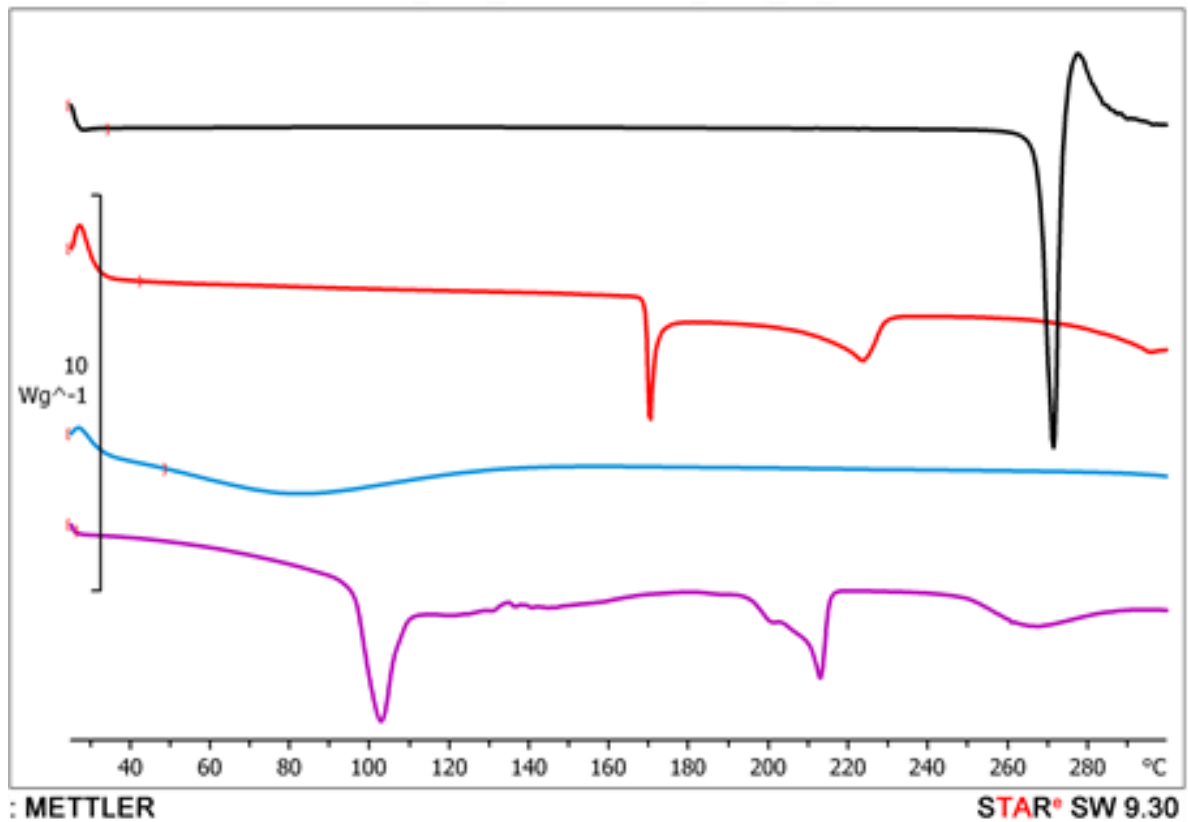

(A) 


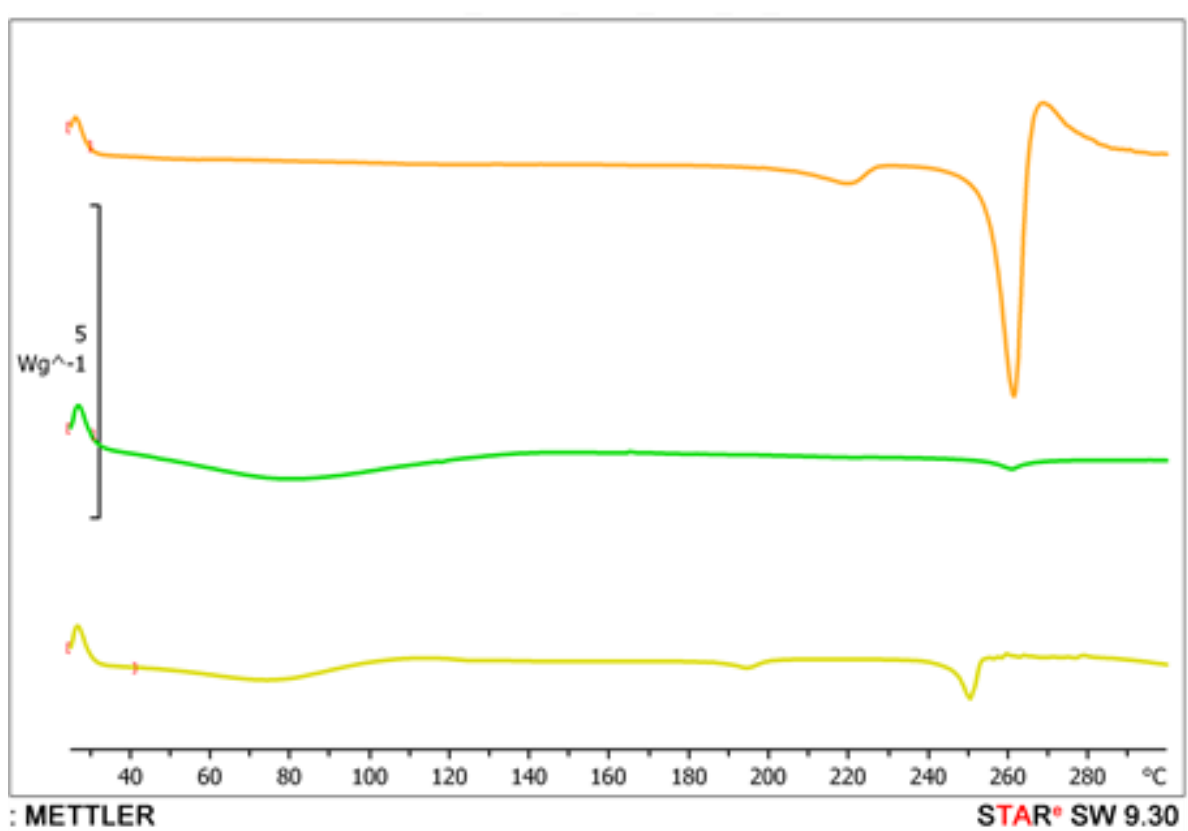

(B)

3 Figure 3 DSC curves of rawMel (black), PVA (red), MCC (blue) and trehalose (purple) (A), and nanoMel

4 (orange) fluidMel (green) and lyoMel (yellow) (B)

Abbreviations: DSC, differencial scanning calorimetry; Mel, meloxicam; PVA, poly(vinyl alcohol)The thermograms of the products are shown in Figure 3b. It is clear, that the melting point and the enthalpy of Mel in the case of the nanoMel decreased due to the partial amorphization. The curve of the fluidMel shows the peak of MCC and the decreased melting point of Mel (Figure 3B). The first obvious and big endothermic peak of the curve appearing from $30^{\circ} \mathrm{C}$ to $150{ }^{\circ} \mathrm{C}$ is mainly related to the absorbed moisture evaporation. ${ }^{43}$ The second endothermic peak is connected to the melting point of $\mathrm{Mel}\left(264^{\circ} \mathrm{C}\right)$ and the enthalpy was decreased due to the large amount of MCC, which covered the characteristic peak of PVA as well, compared to nanoMel. The curve of lyoMel represents the peak of PVA $\left(197^{\circ} \mathrm{C}\right)$ and the melting temperature of Mel $(251$ $\left.{ }^{\circ} \mathrm{C}\right)$ (Figure 3B). According to the literature ${ }^{44}$ and our measurements, during the process, the total amount of trehalose transformed into an amorphous form and the lyophilized trehalose maintains its amorphous form. The big endothermic peak of the curve appearing from $30^{\circ} \mathrm{C}$ to $150{ }^{\circ} \mathrm{C}$ is connected to the absorbed water evaporation as well.

The crystallinity of Mel in nanoMel and the transformed solid-state products was calculated by the enthalpy changes of the drug occurring during the DSC measurement (Table 3). Each sample was compared to its own physical mixture. According to the crystallinity of the 
nanoMel (13.43\%) sample, the crystallinity of the fluidMel sample did not change (12.98\%), for

2 the lyoMel sample partial recrystallization (40.11\%) occurred.

3 Table 3 Enthalpy and calculated crystallinity values of the characteristic peak of Mel in the samples

\begin{tabular}{|c|c|c|c|}
\hline Sample & Enthalpy & Crystallinity of Mel & Crystallinity of Mel \\
& $(\mathrm{J} / \mathrm{g})$ & $(\%)$ & after 6 months of storage \\
& & & \\
\hline nanoMel & 12.24 & 13.43 & 13.02 \\
\hline fluidMel & 11.83 & 12.98 & 40.16 \\
\hline lyoMel & 36.54 & 40.11 & \\
\hline
\end{tabular}

Abbreviation: Mel, meloxicam

After 6 months of storage $\left(23 \pm 2{ }^{\circ} \mathrm{C}, 45 \pm 5 \% \mathrm{RH}\right)$, the degree of crystallinity of solidified

6

7

9

\section{Drug content determination} sample (lyoMel) was $7.12 \mathrm{mg}$.

The theoretical drug content was $7.50 \mathrm{mg}$ as single dose/oral. For the nanoMel sample this amount was $7.12 \mathrm{mg}$ and fluidMel showed $6.83 \mathrm{mg}$ of Mel. The latter can be related to the yield of the fluidization technique (95.93\%). During the lyophilization process the Mel content of the

Solubility testing of MEL in the samples

The solubility of nanoMel increased significantly $(9.4 \pm 0.5 \mu \mathrm{g} / \mathrm{ml})$ in comparison with the rawMel $(6.5 \pm 0.2 \mu \mathrm{g} / \mathrm{ml})$. The reduced particle size enhanced the wettability of the hydrophobic particle when using PVA, therefore increased the thermodynamic solubility of Mel. The fluidization 
1 process did not affect the solubility of Mel $(9.6 \pm 0.4 \mu \mathrm{g} / \mathrm{ml})$. In the case of lyoMel, solubility was

2 increased $(11.2 \pm 0.5 \mu \mathrm{g} / \mathrm{ml})$ because of the presence of trehalose.

3

\section{$4 \quad$ In vitro dissolution studies}

5 The in vitro dissolution extent of the samples was investigated in gastric juice $(\mathrm{pH}=1.2)$. Mel has

6 a week acidic character, therefore its solubility in this medium is very poor $(1.6 \pm 0.2 \mu \mathrm{g} / \mathrm{ml}$, at 37

$7 \quad{ }^{\circ} \mathrm{C}$ ). Figure 4 clearly demonstrates that the particle size reduction of Mel in the nanosuspension

8 (nanoMel) influenced the dissolution rate of Mel, but resulted in only $40 \%$ of drug release in 5

9 minutes, and then the curve took a stagnant profile. The initial rapid drug release can be

10 associated with the nanoscale Mel and its amorphous structure. The 2-hour test did not result in any more favorable results. Although the distribution of the nanoparticles of Mel in the nanosuspension is suitable, a large volume of acidic medium (900 ml) may increase the aggregation of the nanoparticles. In this case, the protective effect of the polymer (PVA) is unsatisfactory.

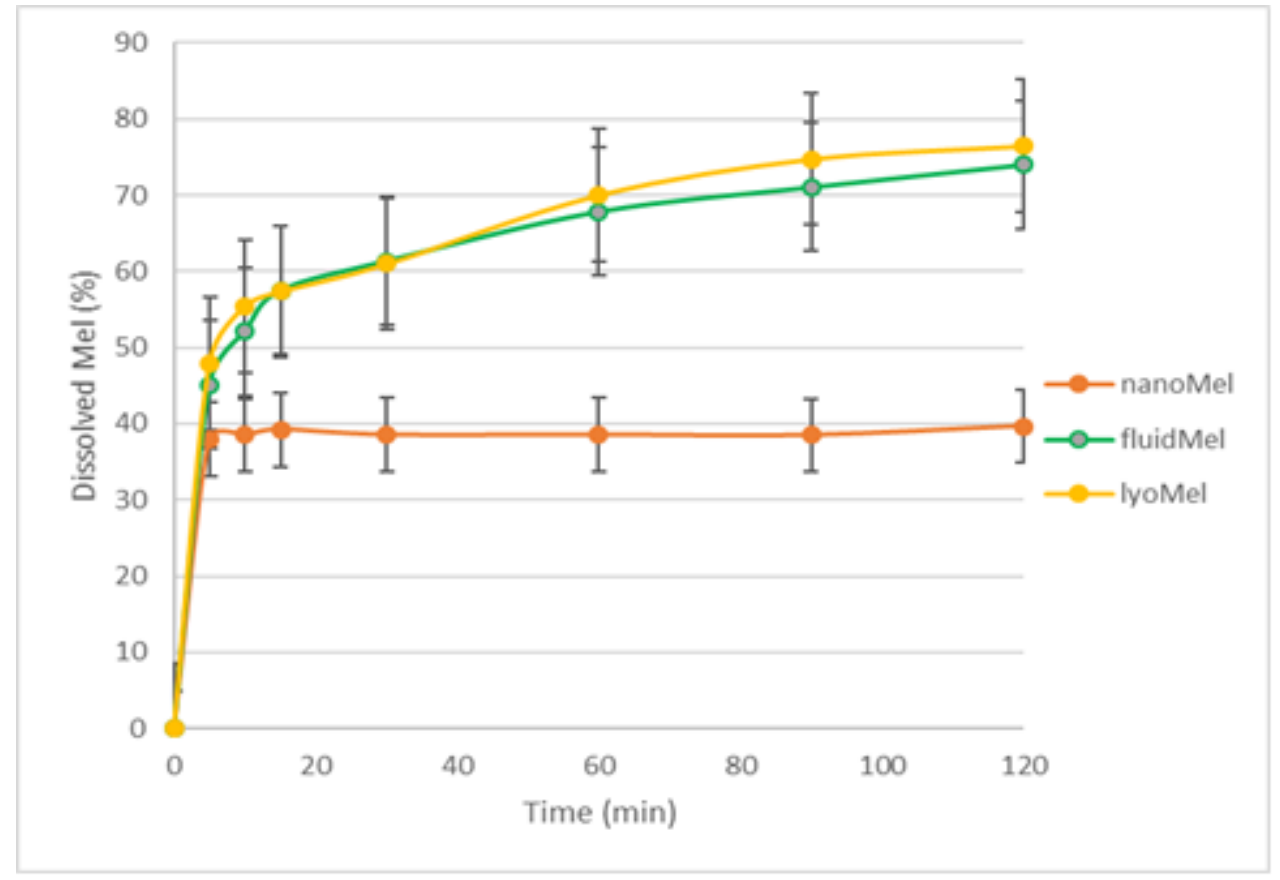

Figure 4 In vitro dissolution of Mel from investigated samples. Medium: artificial gastric juice (pH: 1.2)

Abbreviations: Mel, meloxicam

For fluidMel and lyoMel samples, a rapid initial phase is observed (about $60 \%$ of the drug is dissolved in 15 minutes), followed by a slowing but rising profile. About $75 \%$ of Mel was 
1 dissolved within 2 hours. In the case of the solid state forms, carriers (MCC and trehalose) help

2 to uniformly distribute the nanoparticles of Mel, thereby maintaining the uniqueness of the nanoparticles.

4

\section{$5 \quad$ In vivo studies}

6 The plasma concentration of the samples in rats is shown in Figure 5. The calculated plasma

7 concentration of Mel at zero $\min \left(\mathrm{C}_{0 \mathrm{~min}}\right)$ was $10,607 \mathrm{nM}$, and then the plasma concentration

8 decreased exponentially. A very small amount of Mel was absorbed from nanoMel sample,

9 regardless of the particle size of the drug. The plasma concentration of Mel was constant in the

10 investigated time period. The results show that the nanosuspension (nanoMel) has not got advantageous properties.

12

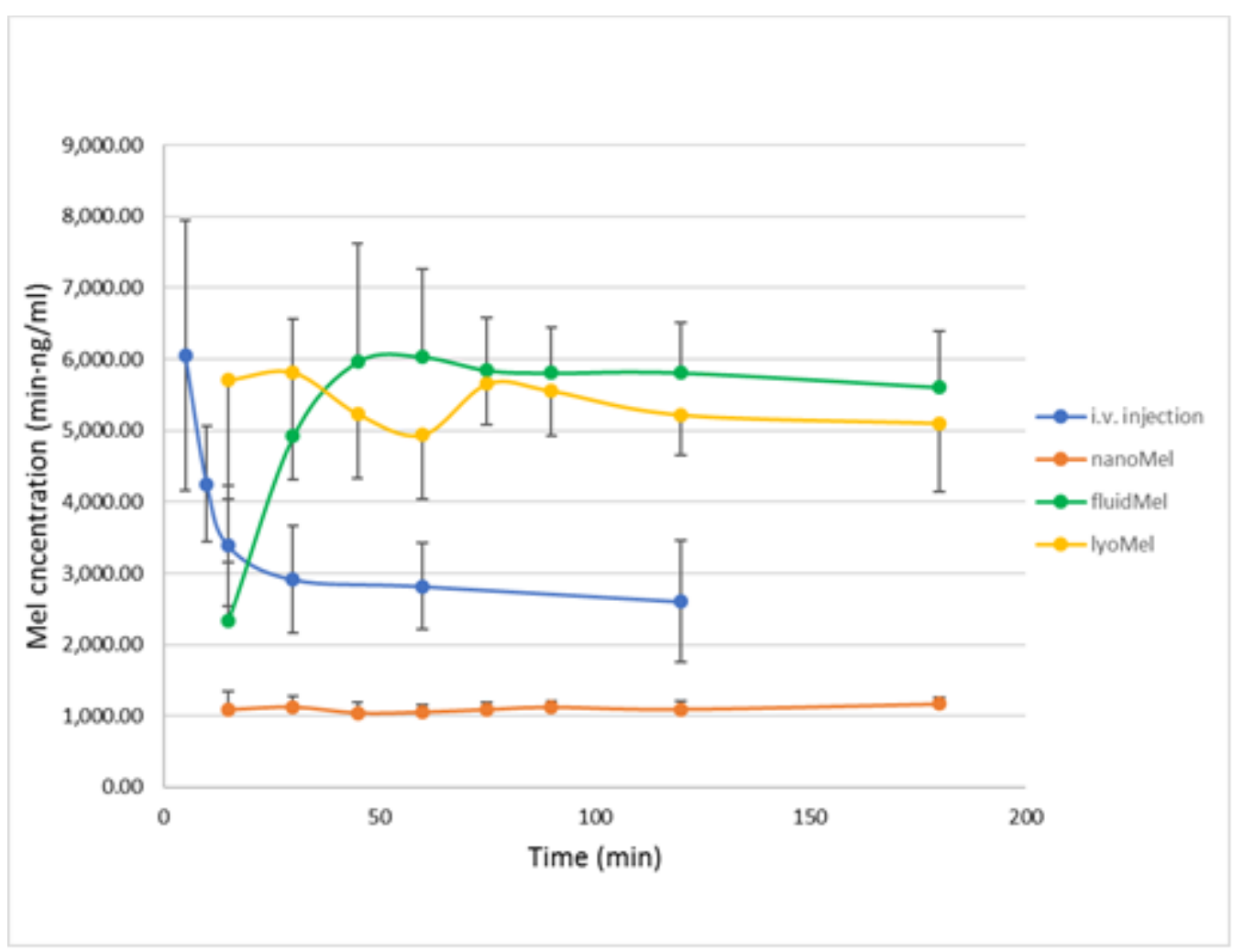

Figure 5 Plasma levels of MEL after the administration of different samples in rats. The preparations were administered orally (nanoMel, fluidMel and lyoMel) or intravenously (IV) as a single dose of 300 $\mu \mathrm{g} / \mathrm{kg}$

\section{Abbreviations: Mel, meloxicam}

The initial blood levels of the fluidMel and lyoMel samples show a big difference. At 15 minutes, the lyoMel sample $(5,712.98 \mathrm{nM})$ shows more than twice the value of fluidMel (Table 4). 
1 In practice, this value is similar as the maximum plasma level for the lyophilized product $\left(\mathrm{C}_{30 \mathrm{~min}}\right.$

$25,814 \mathrm{nM}$ ). The peak blood concentration of fluidMel, is about $6,000.00 \mathrm{nM}$ at 50 minutes that is

3

4

5

6

7

8

9

10

11

12 comparable with the blood concentration reached by IV injection at $5 \mathrm{~min}$. This result also confirms that the solid products contained Mel in an adequate amount, and that the total amount thereof dissolved and absorbed.

The plasma curves of the different samples containing Mel show a very slow elimination after the distribution phases. That can be explained by the very high $(99 \%)$ plasma binding property of Mel in rat, and this ratio is the same in human. ${ }^{45}$ It seems that the eliminated portion of $\mathrm{Mel}$ is replenished from the protein bounded fraction for a quite long period of time. Our measuring time was only 3 hours, longer detection period can provide appropriate information about the whole elimination process. The peak MEL concentrations of from lyoMel and fluidMel preparations have reached the similar level that of IV formula (Figure 5). The lyoMel sample resulted in higher plasma concentrations in 15 minutes as compared with nanoMEL preparation. The solidified samples had nearly five-fold higher bioavailability than that of nanoMel (Table 4).

Table 4 Plasma concentrations of Mel in time and its relative bioavailability in rats after IV and per os administration of Mel samples. Relative bioavailabilities were compared to nanoMel preparation.

\begin{tabular}{|l|l|l|l|l|}
\hline Sample & $\mathrm{C}_{15 \mathrm{~min}}$ & $\mathrm{C}_{120 \mathrm{~min}}$ & $\mathrm{AUC}_{\text {blood }}$ & Relative \\
& $(\mathrm{nM})$ & $(\mathrm{nM})$ & $(\mathrm{min} \cdot \mathrm{ng} / \mathrm{ml})$ & bioavailability (\%) \\
\hline nanoMel & $1,090.02 \pm 13.11$ & $1,123.31 \pm 14.24$ & $190,584.52$ & 100.00 \\
\hline fluidMel & $2,338.44 \pm 17.25$ & $5,811.33 \pm 18.34$ & $945,834.99$ & 496.28 \\
\hline IyoMel & $5,712.98 \pm 28.36$ & $5,219.52 \pm 20.86$ & $923,117.95$ & 484.36 \\
\hline IV injection & $C_{5 \min }$ & & & \\
\hline & $6,059.07 \pm 15.76$ & & $377,528.01$ & - \\
\hline
\end{tabular}

Abbreviations: Mel, meloxicam; $C_{15 \min }, C_{120 m i n}$, plasma concentrations of Mel in time; $A U C_{b l o o d}$, area under the time-concentration curve AUC interval is $\mathrm{t}_{0 \min }-\mathrm{t}_{180 \mathrm{~min}}$ 


\section{IVIV correlation}

2 Comparative studies according to AUC values have shown that there are significant differences

3 between the nanosuspension and the samples (fluidMel and lyoMel) within in vitro and in vivo

4 groups. However, there is no significant difference between two solid samples either in vitro or in vivo (Figure 6). The basis of the IVIVC calculation was the comparison of the AUC values of the samples in the in vitro and in vivo groups. By our calculations, the Pearson's correlation coefficient value between the two studies is 0.99695 . The $t$ value of the independent $t$-test of the two dissolution study series was 0.0145 , the calculated $p$ value was 0.9889 and the difference is not significant at a confidence level of $95 \%$. As the zero hypothesis of the independent t-test, the calculation is not significant if the averages of the two series are equal. It can be concluded that in this system, in vitro dissolution studies are applicable to predict the dissolution rate-limited differences in the case of in vivo studies.

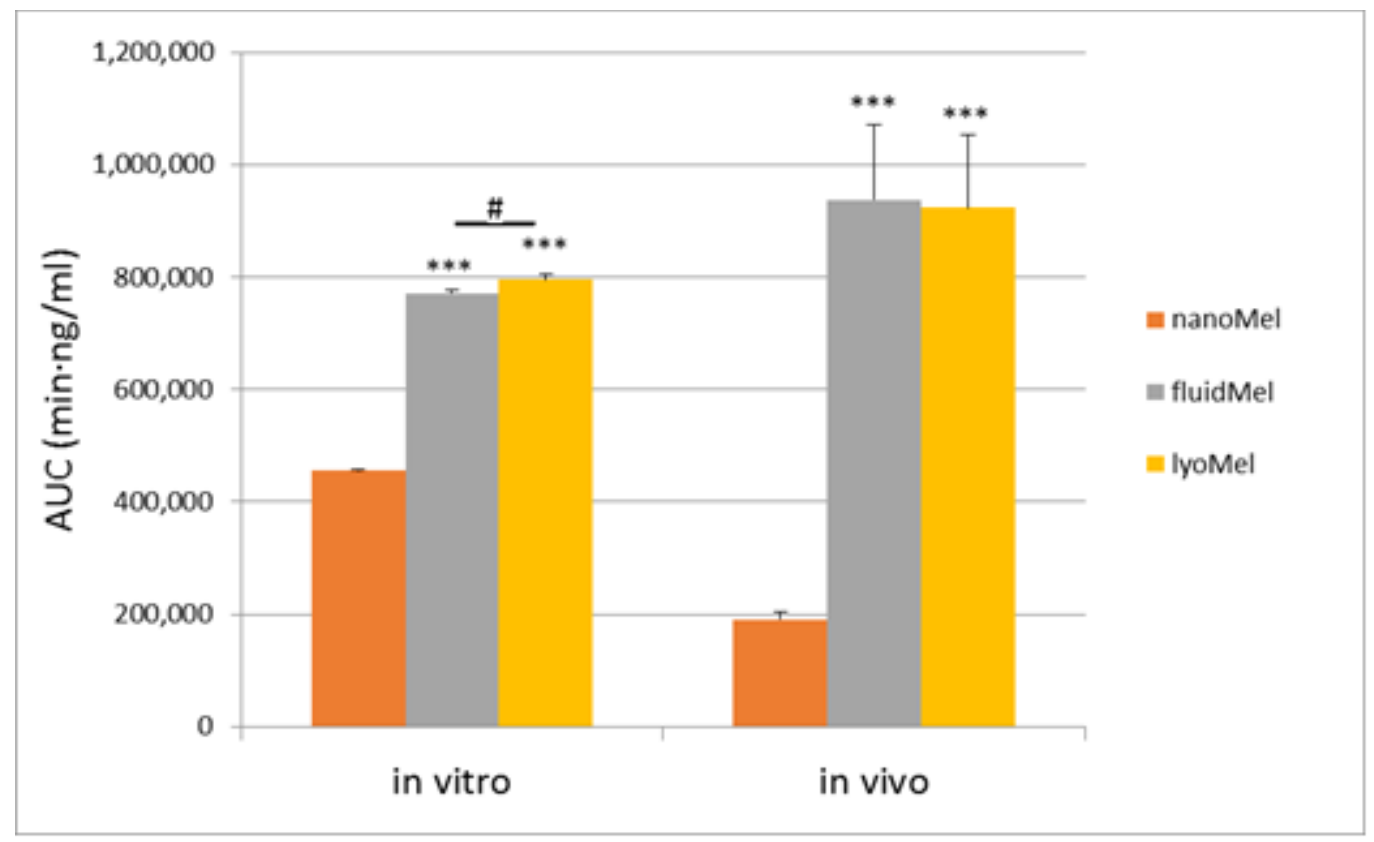

Figure 6 IVIV correlation of Mel containing samples. Notes: Values are presented as mean \pm SD. Statistically significant differences are: ${ }^{* * *} p<0.001$, compared to nanoMel separately in in vitro and in vivo groups; \# $p<0.05$ compared to the indicated columns

Abbreviations: Mel, meloxicam; AUC, area under the time-concentration curve; IVIV correlation, in vitro-in vivo correlation; SD, standard deviation 
Mel-containing surfactant-free nanosuspension (nanoMel) as intermediate product was produced by wet milling process (planetary ball mill was combined with pearl milling technology). The energy invested in the milling decreased the mean particle size of Mel $(130 \mathrm{~nm})$ and broke the crystal structure of the nanoparticles (crystallinity index: $13.43 \%$ ). This can be considered a labile system, so stabilizing a suspension as a final dosage form without a surfactant does not work, therefore the solidification of the nanoMel produced by fluidization and lyophilization was chosen to ensure the critical product parameters (particle size, degree of crystallinity). We studied also the influence of solidification of nanoMel on the physical stability and drug bioavailability of the products. The nanoMel product had an optimized amount of PVA $(0.5 \%)$ as a protective polymer, but no surfactant as a further stabilizing agent because the final aim was to produce a surfactantfree solid phase products as well.

The solidification studies showed that the critical product parameters of the intermediate product (nanoMel) were primarily provided by the fluidization technique which resulted no significant change in mean particle size and crystallinity degree of Mel compared to the nanoMel. It is connected to the short operation time (50 min), the large surface area of the MCC which fixed the nanoparticles with the sticking effect of the PVA and the crystallization inhibitory property of the fibers. ${ }^{34}$ The lyophilization required a longer operation time (72 hours), which resulted in the amorphization of the crystalline carrier (trehalose) ${ }^{46}$ and the recrystallization of Mel with an increased particle size and crystallinity degree. Finally it was found that the physical stability of the solid phase products (fluidMel, lyoMel) was no change in particle size and crystallinity at 6 months of storage at room temperature $\left(23 \pm 2{ }^{\circ} \mathrm{C}, 45 \pm 5 \% \mathrm{RH}\right)$ compared to freshly measured products.

In our pervious work (DDDT, 2018) ${ }^{10}$, there was performed a human Caco-2 intestinal epithel cell line viability assay. Impedance measurement did not show significant cell damage after treatments with Mel, PVA-Mel formulations, as reflected by unchanged cell index values. The epithelial electrical resistance studies predicted the rapid penetration of nanonized Mel. In vivo studies justified the predicted data. The nanonized Mel in solidified products (fluidMel, lyoMel) resulted in rapid absorption through the gastric membrane by passive transcellular transport. It was found that the solid products contained Mel in an adequate amount, and that the total amount 
1 thereof dissolved and absorbed. It has to be noted that the Mel shows a very slow elimination

2 because of very high plasma binding (99\%) in rat, and its replenishing time is quite long period.

3 MEL blood concentrations of lyoMel and fluidMel were similar as level of IV form.

4

The solidified samples had nearly five-fold higher relative bioavailability than nanoMel

5

6

7

8

\section{Acknowledgments}

This work was supported by the National Research Development and Innovation Office and application by oral administration and IVIV correlation was found between the in vitro and in vivo studies. The correlation between in vitro and in vivo studies showed that Mel nanoparticles fixed on solid carrier (MCC, trehalose) in both the artificial gastric juice and the stomach of the animals rapidly reach saturation concentration leading to rapid absorption. These products show about 5 times greater bioavailability than the nanosuspension, in which the Mel nanoparticles can be aggregated in the stomach.

It can be stated that in the present study the solidification of the nanosuspension (nanoMel) not only increased the stability of the nanoparticles, but also allowed the preparation of surfactant-free solid compositions (powder, tablet, capsule), which may be an important consideration for certain groups of patients to achieve rapid analgesia. Further experiments are necessary to prove the therapeutic relevance of these innovative formulations.

Richter Plc., Budapest, Hungary (GINOP 2.2.1 15-2016-00007).

The grant (20391-3/2018/FEKUSTRAT) of the Ministry of Human Capacities, Hungary is acknowledged.

The help of Tamás Sovány assistant professor with fluidization technique is gratefully acknowledged. 


\section{Author contributions}

2 All authors contributed to data analysis, drafting or revising the article, gave final approval of the

3 version to be published, and agree to be accountable for all aspects of the

4 work.Disclosure

5 The authors report no financial interest and no conflicts of interest in this work. 


\section{References}

21 Müller RH, Benita S, Böhm BHL. Emulsions and nanosuspensions for the formulation of

3 poorly soluble drugs. Medpharm Scientific Publishers, Stuttgart, Germany, 1998, 149-174

42 Dahiya S, Pathak K, Dahiya R, et al. Nanoparticle engineering processes: Industrial

5 technologies for delivery of poorly water-soluble drugs. Egypt. Pharm. J. 2007;6:87-101.

63 Meng L, Mohammad A, Rajesh D, et al. Nanomilling of Drugs for Bioavailability

7 Enhancement: A Holistic Formulation-Process Perspective. Pharmaceutics, 2016;8,17-52.

84 Sharma P, denny WA, Garg S. Effect of wet milling process on the solid state of

9 indomethacinn and simvastatin. Int. J. Pharm. 2009;380:40-48.

105 Malamatari M, Somavarapu S, Taylor KMG et al. Solidufication of nanosuspensions for the

11 production of solid oral dosage forms and inhalable dry powders. Exp. Opin. Drug Del.

$12 \quad 2016 ; 13: 435-450$.

136 Lee J, Cheng Y. Critical freezing rate in freeze drying nanocrystal dispersions. J. Control.

14 Release 2006;111:185-192.

157 Liversidge G G, Cundy K C. Particle size reduction for improvement of oral bioavailability of

16 hydrophobic drugs. I. Absolute oral bioavailability of nanocrystalline danazol in beagle dogs.

17 Int. J. Pharm. 1995;125:91-97.

188 Ding Z, Wang L, Xing Y, Zhao Y, Wang Z, Han J. Enhanced Oral Bioavailability of Celecoxib

19 Nanocrystalline Solid Dispersion based on Wet Media Milling Technique: Formulation,

20 Optimization and In Vitro/In Vivo Evaluation. Pharmaceutics 2019;11:328-346. 
19 Wiedmann T S, DeCastro L, Wood R W. Nebulization of NanoCrystalsTM: production of a

2 respirable solid-in-liquid-in-air colloidal dispersion. Pharm. Res. 1997;14:112-116.

310 Bartos C, Jójárt-Laczkovich O, Katona G, et al. Optimization of a combined wet milling

4 process in order to produce poly(vinyl alcohol) stabilized nanosuspension. Drug Des. Dev. Ther.

$5 \quad 2018 ; 12: 1567-1580$.

611 Chiang P C, Wahlstrom J L, Selbo J G et al. 1,3- Dicyclohexyl urea nanosuspension for

7 intravenous steady-state delivery in rats. J. Exp. Nanosci. 2007;2:239-250.

812 Zhang Y, Fei S, Yu M et al. Injectable sustained release PLA microparticles prepared by

9 solvent evaporation-media milling technology. Drug Dev. Ind. Pharm. 2018;44:1591-1597.

1013 Girdhar V, Patil S, Banerjee S, Singhvi G. Nanocarriers For Drug Delivery: Mini Review.

11 Recent Pat. Nanomed. 2018;8:88-99.

1214 Ostrander K D, Bosch H W, Bondanza D M. An in-vitro assessment of a NanoCrystalTM

13 beclomethasone dipropionate colloidal dispersion via ultrasonic nebulization. Eur. J. Pharm.

14 Biopharm. 1999;48:207-215.

1515 Vergote G J, Nervaet C, Van Driessche I, Hoste S, De Smedt S, Demeester J, Jain R A, Ruddy

16 S, Remon J P . An oral controlled release matrix pellet formulation containing nanocrystalline

17 ketoprofen, Int. J. Pharm. 2001;2019:81-87. S, Polin L, Jones J, Corbett T, Cooper E, Liversidge G G. Formulation and antitumor activity 
1 evaluation of nanocrystalline suspensions of poorly soluble anticancer drugs. Pharm Res.

2 1996;13:272-278.

317 Siewert C, Moog R, Alex R, Kretzer P, Rothenhäusler B. Process and scaling parameters for

4 wet media milling in early phase drug development: A knowledge-based approach. Eur J Pharm

5 Sci. 2018;115:126-131.

618 Peltonen L, Hirvonen J. Pharmaceutical nanocrystals by nanomilling: critical process

7 parameters, particle fracturing and stabilization methods. J Pharm Pharmacol. 2010;62:1569-1579.

819 Kim CJ. Surface chemistry and colloids in Advanced pharmaceutics: physico-chemical

9 principles, CRC Press, Florida, United States of America, 2004, 193-256.

1020 Derjaguin B V, Landau L. Theory of the stability of strongly charged lyophobic sols and of

11 the adhesion of strongly charged particles in solutions of electrolytes. Acta Phys Chim.

$12 \quad 1941 ; 14: 633-662$.

1321 Verma S, Kumar S, Gokhale R, J.Burgess D. Physical stability of nanosuspensions:

14 Investigation of the role of stabilizers on Ostwald ripening. Int J Pharm. 2011;406:145-152

1522 Bhakay A, Azad M, Vizzotti E, et al. Enhanced recovery and dissolution of griseofulvin

16 nanoparticles from surfactant-free nanocomposite microparticles incorporating wet-milled

17 swellable dispersants. Drug Dev Ind Pharm. 2014;40:1509-1522. 
124 Hye_in K, Sang YP, Seok JP, et al. Development and evaluation of a reconstitutable dry

2 suspension ti improve the dissolution and oral absorption of poorly water-soluble celecoxib.

3 Pharmceutics 2018;10: 140, doi:10.3390/pharmaceutics10030140

$425 \mathrm{Wu}$ L, Zhang J, Watanabe W. Physical and chemical stability of drug nanoparticles. Adv Drug

$5 \quad$ Deliv Re. 2011;63:456-469.

626 Wang Y, Zheng Y, Zhang L, et al. Stability of nanosuspensions in drug delivery. J Control

$7 \quad$ Release. 2013;172:1126-1141.

827 Van Eerdenbrugh B, Froyen L, Van Humbeeck J, et al. Drying of crystalline drug

9 nanosuspensions - The importance of surface hydrophobicity on dissolution behavior upon

10 redispersion. Eur J Pharm Sci. 2008;35:127-135.

1128 Kumari Prasad L, Smyth H. 3D Printing technologies for drug delivery: a review. Drug Dev

12 Ind Pharm. 2016;42: 1019-1031.

1329 Colombo M, Orthmann S, Bellini M et al. Influence of drug brittleness, nanomilling time, and

14 freeze-drying on the crystallinity of poorly water-soluble drugs and its implications for

15 solubility enhancement. AAPS Pharm Sci Tech. 2017;18:2437-2445.

1630 Kayaert P, Anné M, Van den Mooter G. Bead layering as a process to stabilize

17 nanosuspensions: influence of drug hydrophobicity on nanocrystal reagglomeration following

18 in-vitro release from sugar beads. J Pharm Pharmacol. 2011;63:1446-53

$1931 \mathrm{He} \mathrm{W,} \mathrm{Lu} \mathrm{Y,} \mathrm{Qi} \mathrm{J,} \mathrm{et} \mathrm{al.} \mathrm{Formulating} \mathrm{food} \mathrm{protein-stabilized} \mathrm{indomethacin} \mathrm{nanosuspensions}$

20 into pellets by fluid-bed coating technology: physical characterization, redispersibility, and

21 dissolution. Int J Nanomedicine. 2013;8:3119-3128. 
132 Möschwitzer J, Müller RH. Spray coated pellets as carrier system for mucoadhesive drug

2 nanocrystals. Eur J Pharm Biopharm. 2006;62:282-287.

333 Vergote G, Vervaet C, Van Driessche I, et al. An oral controlled release matrix pellet

4 formulation containing nanocrystalline ketoprofen. Int J Pharm. 2001;219:81-87.

534 Jójárt-Laczkovich O, Szabó-Révész P. Formulation of tablets containingan 'in-process'

6 amorphized active pharmaceutical ingredient, Drug Dev Ind Pharm. 2011;37:1272-1281.

$7 \quad 35$ Szabó-Révész P. Modifying the physicochemical properties of NSAIDs for nasal and

8 pulmonary administration. Drug Discov Today: Technol. 2018; 27: 87-93.

936 Simmons DL, Botting RM, Hla T. Cyclooxygenase isozymes: the biology of prostaglandin

10 synthesis and inhibition. Pharmacol Rev. 2004; 56: 387-437.

1137 Bartos Cs, Szabó-Révész P, Bartos C, et al. The Effect of an Optimized Wet Milling

12 Technology on the Crystallinity, Morphology and Dissolution Properties of Micro- and

13 Nanonized Meloxicam. Molecules 2016;21:507-518.

1438 Abramoff MD, Magelhaes PJ, Ram SJ. Image processing with image. J Biophotonics Int.

$15 \quad 2004 ; 11: 36-42$.

1639 Mártha C, Kürti L, Farkas G et al. Effects of polymers on the crystallinity of nanonized

17 meloxicam during a co-grinding process. Eur Polym J. 2013;49:2426-2432

1840 Aulton ME, Livingstone C. Aulton's Pharmaceutics, The Design and Manufacture of

19 Medicines. Elsevier 2007.

2041 Cardot J M, Davit B M. In vitro-In Vivo Correlations: Tricks and Traps. AAPS J. 2012;14:491-

21499. 
142 Kevin J. Using Nondestructive in-situ Measurements to Ensure Lyophilized Product

$2 \quad$ Stability. Biopharm Int. 2018;31:24-25.

343 Kian LK, Jawaid M, Ariffin H, et al. Isolation and characterization of microcrystalline

4 cellulose from roselle fibers. Int Biol Macromol. 2017;103:931-940.

544 Sussich F, Cesàro A. Trehalose amorphization and recrystallization. Carbohyd Re.

$6 \quad 2008 ; 343: 2667-2674$.

745 Busch U, Schmid J, Heinzel G, et al. Pharmacokinetics of Meloxicam in Animals and The

8 Relevance to Humans. Drug Metab Dispos. 1998;26:576-584.

946 Jójárt-Laczkovich O, Katona G, Aigner Z, et al. Investigation of recrystallization of

10 amorphous trehalose through hot-humidity stage X-ray powder diffraction. Eur J Pharm Sci.

$112016 ; 95: 145-151$. 